\title{
Adaptive Scanning for STED Microscopy
}

\author{
Dissertation
}

zur Erlangung des mathematisch-naturwissenschaftlichen Doktorgrades

„Doctor rerum naturalium“

der Georg-August-Universität Göttingen

im Promotionsprogramm ProPhys

der Georg-August University School of Science (GAUSS)

vorgelegt von

\section{Britta Vinçon}

aus Rottweil

Göttingen, 2019 
Betreuungsausschuss

apl. Prof. Dr. Alexander Egner

Optische Nanoskopie

Laser-Laboratorium Göttingen e.V.

Prof. Dr. Sarah Köster

Institut für Röntgenphysik

Georg-August-Universität Göttingen

Mitglieder der Prüfungskommission

Referent: apl. Prof. Dr. Alexander Egner

Korreferentin: Prof. Dr. Sarah Köster

Weitere Mitglieder der Prüfungskommission:

\section{Prof. Dr. Jörg Enderlein}

III. Physikalisches Institut

Georg-August-Universität Göttingen

\section{Prof. Dr. Wolfram Kollatschny}

Institut für Astrophysik

Georg-August-Universität Göttingen

\section{Prof. Dr. Silvio O. Rizzoli}

Abteilung für Neuro- und Sinnesphysiologie

Universitätsmedizin Göttingen

apl. Prof. Dr. Michael Seibt

IV. Physikalisches Institut

Georg-August-Universität Göttingen

Tag der mündlichen Prüfung: 31. Januar 2020 


\section{Abstract}

Optical nanoscopy allows for highly specific imaging of biological tissue, cellular components and even single molecules and has therefore become an integral part of modern biomedical research. In STED microscopy, being one of these techniques, super-resolution is achieved by employing a depletion intensity distribution to confine the fluorescence to a sub-diffraction sized area [Hel07]. The high focal intensities typically employed for an efficient depletion of fluorophores can however cause phototoxicity and photodamage to the sample [Hel03]. Several techniques have been presented recently to tackle this problem. They are based on avoiding the transition of dye molecules to any other than one of the desired molecular states or on reducing the light dose by lowering the employed laser power or effective illumination time. For STED microscopy, however, this reduction has not yet been translated into a faster acquisition time, which would be of particular advantage for the investigation of fast biological processes. Another technique that reduces both light dose and acquisition time is tomoSTED microscopy, which drastically reduces photobleaching and sample damage [Krü17]. But even this form of STED microscopy could benefit from an adaptation of the scanning process.

Within this work, adaptive scanning as an approach to locally adjust the total pixel dwell time and/or the local intensity distribution is developed and evaluated for its potential to improve the performance of STED microscopy with respect to the beforehand outlined aspects. For this purpose, a scan system with a fast response time is developed, implemented and characterized. Additionally, a novel method to generate 1D depletion patterns for tomoSTED microscopy based on conical diffraction is introduced, for which the pattern orientation can be switched on the single pixel level by using electro-optical devices, paving the way to employing tomoSTED microscopy with adaptive scanning.

Utilizing the new scan system, a novel technique called FastRESCue, introduced here as a variant of RESCue [SER $\left.{ }^{+} 11\right]$, proves to allow a direct translation of a reduced light dose into faster image acquisition, yielding imaging at only $20 \%$ of both light dose and acquisition time at uncompromised image quality and resolution. A further reduction of the acquisition time is realized by adapting the scanned area directly to the sample structure under investigation. Employing the novel 1D depletion patterns for tomoSTED microscopy, this innovative adaptive scanning technique enables a real-time detection of the structure's position and orientation. It is therefore successfully applied in tracing of filamentous structures in both fixed and living cells.

In conclusion, this thesis demonstrates the successful application of adaptive scanning for STED microscopy with the focus on low-illumination and fast-acquisition imaging schemes. The extension of this concept to e.g. three-dimensional scanning or other sample structures will remain the focus of future work. 



\section{Contents}

\begin{tabular}{lll}
\hline List of Figures & VII
\end{tabular}

\begin{tabular}{ll}
\hline 1. Introduction & 1
\end{tabular}

2. Theoretical Framework 5

2.1. Introduction to STED microscopy . . . . . . . . . . . . . . 5

2.1.1. Abbe's diffraction limit . . . . . . . . . . . . . . . . . 5

2.1.2. Breaking the diffraction limit . . . . . . . . . . . . . 8

2.2. Tomographic STED microscopy $\ldots \ldots \ldots \ldots \ldots \ldots$. . . . . . . . . . . . . 12

2.2.1. Resolution increase along one direction . . . . . . . . . . . . . . 12

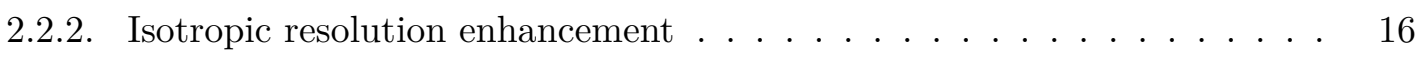

2.3. Sample-conserving imaging techniques . . . . . . . . . . . . . . . . . . . . 19

2.4. Light interaction with crystalline materials . . . . . . . . . . . . . . 21

2.4.1. Description of polarization states . . . . . . . . . . . . . . . . . . . . . . . . .

$2.4 .2 . \quad$ Birefringence . . . . . . . . . . . . . . . . . . 24

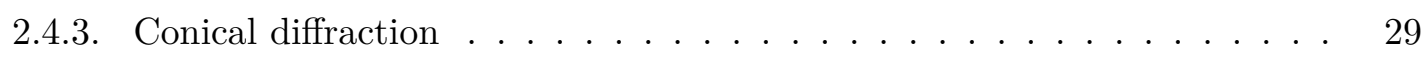

2.4.4. Electro-optic effect . . . . . . . . . . . . . . . . 33

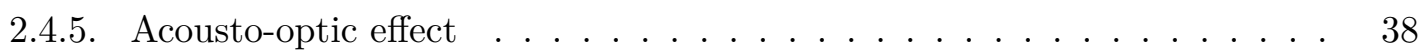

3. Material and Methods 41

3.1. Experimental realization . . . . . . . . . . . . . . . . . . . . 41

3.2. Pattern generation . . . . . . . . . . . . . . . . . . 45

3.3. Generation of polarization states $\ldots \ldots \ldots \ldots \ldots$

3.4. Reconstruction algorithms . . . . . . . . . . . . . . . . . . . . . . . . . . . . . 57

3.4.1. Noise correction for oversampling . . . . . . . . . . . . . . . 57

3.4.2. Maximum-value reconstruction . . . . . . . . . . . . . . . . 58

3.4.3. Richardson-Lucy deconvolution . . . . . . . . . . . . . . . . . 60

3.5. Adaptive scanning . . . . . . . . . . . . . . . . . . . . . 63

3.5.1. Galvanometer scanner . . . . . . . . . . . . . . . . . . 64

3.5.2. Electro-optic deflectors . . . . . . . . . . . . . . . . . . . . . . 67

$3.5 .3 . \quad$ Assembly of the combined scan system . . . . . . . . . . . . . 69

3.5.4. LabVIEW program. . . . . . . . . . . . . . . . 70

\begin{tabular}{ll}
\hline 4. Results and Discussion & $\mathbf{7 5}$
\end{tabular}

4.1. TomoSTED microscopy with pixel-wise switching . . . . . . . . . . . 75

4.1.1. Imaging of fluorescent microspheres . . . . . . . . . . . . 75

4.1.2. Imaging of microtubules in fixed Vero cells . . . . . . . . . . . . 79 
4.2. FastRESCue $\ldots \ldots \ldots \ldots \ldots$

4.2.1. Characterization of the EOD-assisted scan system . . . . . . . . . 82

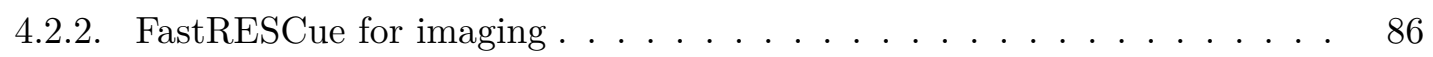

$4.3 . \quad$ Filament tracing $\ldots \ldots \ldots \ldots \ldots \ldots \ldots$

4.3 .1$. Tracing of simulated structures $\ldots \ldots \ldots . \ldots \ldots 9$

$4.3 .2 . \quad$ Tracing of actin filaments $\ldots \ldots \ldots \ldots$. . . . . . . . . . . . . . . . . . . 98

4.3.3. Tracing of microtubules in fixed fibroblasts . . . . . . . . . . . . . . . . 99

4.3.4. Tracing of microtubules in living fibroblasts . . . . . . . . . . . . . 102

$\begin{array}{ll}\text { 5. Conclusion and Outlook } & 105\end{array}$

\begin{tabular}{ll}
\hline References & 111
\end{tabular}

\begin{tabular}{ll}
\hline Nomenclature and Abbreviations & 119
\end{tabular}

\begin{tabular}{|l|l|}
\hline A. Appendix: Mathematical description of a galvanometer & 123
\end{tabular}

\begin{tabular}{ll}
\hline B. Appendix: Sample preparation & 125
\end{tabular} 


\section{List of Figures}

2.1. Illustration of an illumination PSF $\ldots \ldots \ldots \ldots \ldots$

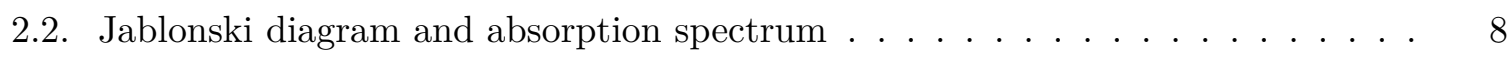

2.3. Comparison of $1 \mathrm{D}$ and $2 \mathrm{D}$ depletion pattern $\ldots \ldots \ldots \ldots \ldots$

2.4. Visualization of 1D and 2D STED OTFS . . . . . . . . . . . . . . 17

2.5. Criteria for the optimal number of pattern orientations . . . . . . . . . . . 18

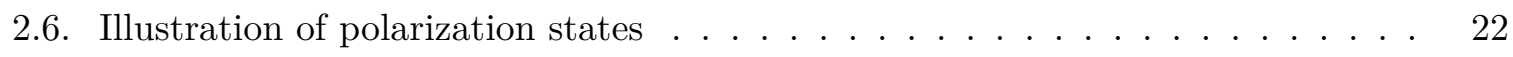

2.7. Index ellipsoid and index ellipse for an anisotropic material . . . . . . . . . 26

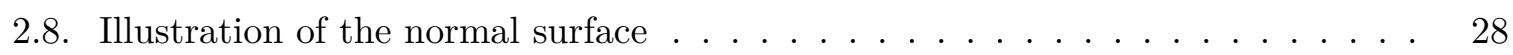

2.9. Sketch of the crystal slab with conically diffracted beam . . . . . . . . . . 30

2.10. Schematic illustration of an electro-optical deflector. . . . . . . . . . . . 35

2.11. Geometry for linear electric field generation by means of quadrupole electrodes 36

2.12. Sketch of an acousto-optic modulator (AOM) . . . . . . . . . . . . 39

3.1. Schematic illustration of the tomoSTED microscope . . . . . . . . . . . . . 42

3.2. Simulation of intensity distributions at $\zeta=0$ for varying $\rho_{0}$ and polarization. . 46

3.3. Simulation of achievable curvature and comparison of intensity profiles . . . . . 48

3.4. Image of the KTP crystal in the setup . . . . . . . . . . . . . . . 49

3.5. Schematic layout for depletion pattern generation . . . . . . . . . . . . . . 49

3.6. Image of a Pockels cells pair. . . . . . . . . . . . . . . . 50

3.7. Illustration of the polarization state generator's working principle . . . . . . . 52

3.8. Exemplary calibration curves for the Pockels cells of the PSG . . . . . . . . . . 54

3.9. Principle of the maximum-value reconstruction $\ldots \ldots \ldots \ldots \ldots$

3.10. Principle of the Richardson-Lucy deconvolution . . . . . . . . . . . . . . . . 62

3.11. Voltage applied by Imspector to the driver of the QUAD scanner . . . . . . . . 65

3.12. Measurement of the scanner's response as a function of the pixel dwell time . . 66

3.13. EOD deflection as a function of the applied control voltage . . . . . . . . 69

3.14. Sketch of the combined scan system's control . . . . . . . . . . . . 70

3.15. Overview of the setup's operation . . . . . . . . . . . . . . 71

3.16. Sketch of the workflow of the FastRESCue LabVIEW FPGA program . . . . . 72

3.17. Sketch of the workflow of the tracing LabVIEW FPGA program . . . . . . 73

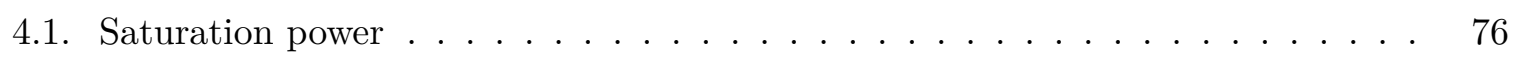

4.2. $\quad$ Dependence of the STED resolution enhancement on $P_{\mathrm{STED}} \ldots \ldots \ldots \ldots$. . . . . . . 77

4.3. TomoSTED microscopy on Crimson 40 microspheres . . . . . . . . . . . . . 78

4.4. TomoSTED image of microtubules in fixed Vero cells . . . . . . . . . . . . 80

4.5. TomoSTED microscopy on microtubules: Imaging at low pixel dwell times. . . 81

4.6. Comparison of different scanning devices: Galvanometer scanner and EODs . . 83

4.7. Comparison of different scan modes: with Imspector and with FPGA . . . . . . 84

4.8. Comparison of different RESCue modes for a 1D depletion pattern . . . . . . . 85 
4.9. FastRESCue for tomoSTED imaging of Crimson 40 microspheres . . . . . . . 87

4.10. FastRESCue with 2D STED microscopy for Crimson 40 microspheres $\ldots . .88$

4.11. FastRESCue: Frame time versus light dose . . . . . . . . . . . . . . 89

4.12. Analysis of the advantage of FastRESCue depending on the size scale . . . . 90

4.13. FastRESCue images of various cellular structures . . . . . . . . . . . . . 91

4.14. Analysis of suitable criteria for determination of a filament's position and direction 93

4.15. Sketch of the scan pattern for the tracing algorithm $\ldots \ldots \ldots$. . . . . . 95

4.16. Results of the first tracing algorithm on a simulated filamentous structure . . . 96

4.17. Results of the optimized tracing algorithm on a simulated filamentous structure 97

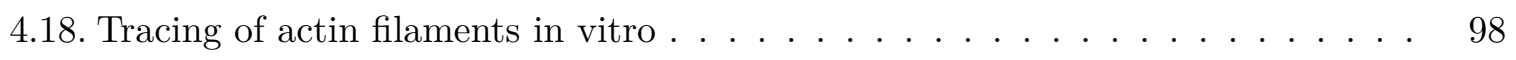

4.19. Tracing of microtubules in fixed fibroblasts $\ldots \ldots \ldots$. . . . . . . . . . . . . . 99

4.20. Comparison of tomoSTED acquisition and filament tracing . . . . . . . . . . 101

4.21. Tracing of microtubules in fixed fibroblasts: several examples . . . . . . . . . 102

4.22. Tracing of microtubules in living fibroblasts . . . . . . . . . . . . . 103 


\section{Introduction}

Ever since its beginnings in the seventeenth century, microscopy has paved the way to groundbreaking discoveries not only in the life sciences, but also e.g. in materials science. Especially far-field fluorescence microscopy has become an essential and versatile tool in the life sciences since it allows non-invasive imaging of sub-cellular structures. Moreover, high molecular specificity is obtained by fluorescence tagging techniques, enabling the distinction of different cellular components. Until the end of the last century, microscopic investigations were limited by diffraction, as first characterized and quantified by Abbe Abb73. Inherent to the wave nature of light, features separated by less than the diffraction limit could not be discerned, independently of the magnification provided by the optical system. Imaging and resolving structures in the range of a few tens of nanometers thus seemed to be impossible with optical microscopy.

Since then, the field of optical microscopy has significantly developed and advanced by exploiting the fluorophores' photophysical properties. Optical switching of molecules between a bright 'on'-state and a dark 'off'-state [Hel03, Hel07] has enabled surpassing the diffraction barrier and pushed the resolution to an unprecedented range. Techniques like PhotoActivatable Localization Microscopy (PALM) [BPS ${ }^{+}$] or STochastic Optical Reconstruction Microscopy (STORM) [RBZ06] are based on a stochastic switching of molecular states, whereas REversible Saturable OpticaL Fluorescence Transitions (RESOLFT) microscopy summarizes several techniques which all rely on defining the area in which the fluorophores are in a specific state via targeted switching of molecules [Hel07]. Depending on the molecular transition utilized, the life times of the employed states differ significantly, ranging from nanoseconds to milliseconds Hel07. It is therefore common practice to use the term RESOLFT mainly for techniques employing switchable proteins [HEJH05] or switchable organic dyes $\mathrm{BFD}^{+} 06$ ], which utilize long-living molecular states. This work deals with a specific targeted sub-diffraction imaging technique, namely STimulated Emission Depletion (STED) microscopy [HW94], but the results can be easily generalized to any other targeted-based imaging schemes.

In STED microscopy, quenching of excited fluorophores by stimulated emission from the first excited state to the ground state is performed by a STED depletion focus, which is red-shifted compared to the excitation beam [Hel07]. For an efficient suppression of fluorescence, the quenching rate needs to outperform the spontaneous decay of the first excited state, resulting in a required intensity in the order of some tens of $\mathrm{MW} / \mathrm{cm}^{2}$ Hel07. Phase-modulating the STED beam yields a typically doughnut-shaped STED focus, which is still diffraction-limited, but features a central intensity minimum. In this way, the area of un-suppressed fluorescence is narrowed, which is equivalent to a resolution enhancement. By increasing the overall laser power, the size of this area can be further reduced, theoretically down to an arbitrarily small spot. Scanning the sample with both co-aligned beams, i.e. the Gaussian-shaped excitation 
and the doughnut-shaped STED beam, allows high-resolution imaging, enabling unprecedented biological discoveries $\left[\mathrm{KWR}^{+} 06\right.$, SHJ17, $\left.\mathrm{DKG}^{+} 15\right]$. However, a doughnut-shaped depletion pattern showing the required intensities close to the central minimum will inevitably yield intensity maxima in the range of $\mathrm{GW} / \mathrm{cm}^{2}$. The light dose employed on the sample during image acquisition with these laser intensities has been repeatedly reported to induce phototoxicity and photodamage to the sample [Hel03, $\left.\mathrm{HKU}^{+} 08, \mathrm{SER}^{+} 11\right]$.

Not only the laser intensity, but also the total acquisition time and thus the pixel dwell time determines the light dose applied to the sample. The latter is typically chosen such that the desired signal-to-noise ratio is reached. An increased resolution, necessitating a smaller pixel size, typically entails a lower fluorescence signal rate per pixel, resulting in a longer pixel dwell time to achieve a decent signal-to-noise ratio. The resulting longer acquisition time, in combination with a higher laser intensity to achieve the increased resolution, typically implies a significantly higher light dose. Moreover, the pixel dwell time does not only affect the light dose, but is directly related to the total acquisition time and thus the maximal acquisition speed. The latter is particularly relevant when investigating fast biological processes at high resolution and is thus as well subject to improvements and optimizations.

Thus, especially in view of the increasing demand for imaging at physiological conditions, a reduction of the light dose and an improvement of the acquisition speed at uncompromised image quality and resolution seems inevitable.

Several approaches have since been pursued in order to reduce the employed light dose in STED microscopy, with the method REduction of State transition Cycles (RESCue) [SER ${ }^{+} 11$ ] being among the first. Here, the reduction is realized by adequately reducing the illumination time in areas of very low or very high signal, but without changing the total acquisition time. A similar principle is the basis of Dynamic Intensity Minimum (DyMIN) [HRH ${ }^{+}$], which employs an adaptive-illumination scheme for optimizing the illumination time per pixel. A different approach is taken in MINFIELD [ $\left.\mathrm{GPH}^{+} 17\right]$, which relies on a reduction of the scan field in order to avoid the illumination of the structure of interest with the maximal intensity of the depletion focus. As neighboring areas do experience these maximal intensities and are thus subject to photodamage, the size of the resulting scan field is inevitably limited and thus not suitable for many biological applications.

For reducing the total acquisition time, parallelization approaches $\mathrm{BAS}^{+} 15, \mathrm{CKG}^{+} 13$ have been conducted, allowing imaging at high acquisition speed without compromising on the resolution as otherwise typically necessary $\left[\mathrm{WRL}^{+} 08\right]$. However, they do not affect the light dose employed on the sample.

A relatively new approach, tackling both the reduction of light dose and total acquisition time, is tomographic STED microscopy (tomoSTED) [Krü17, which has been realized by employing 1D depletion patterns providing only a resolution enhancement in one direction, 
recalling the beginnings of STED microscopy [KEH01]. Those patterns show a higher resolution enhancement at the same laser power as compared to the nowadays commonly utilized doughnut-shaped depletion pattern [KEH01, Krü17] without modifying the scan field or pixel dwell time. Additionally, the collected signal count is higher, enabling a reduction of the total pixel dwell time [Krü17] without compromising on the resolution for the sake of a faster acquisition speed. By an adequate reconstruction of several 1D STED images, a tomoSTED image with two-dimensionally isotropic resolution increase can be obtained [Krü17]. Contrary to the parallelization approaches, tomoSTED microscopy translates the reduction in the total acquisition time in a reduction of the overall light dose employed on the sample.

As demonstrated recently for RESOLFT [ $\left.\mathrm{DCC}^{+} 19\right]$ for pixel dwell times in the order of several hundreds of microseconds, the combination of well-established techniques like RESCue with an adaptable scan pattern can yield a reduction of both the total acquisition time and the light dose. Doing so for much faster techniques like tomoSTED or STED microscopy in general, as already suggested by [SER ${ }^{+} 11$, is intriguing, but not directly accessible. Scan systems currently employed for high-resolution microscopy do not yield the necessary degree of flexibility at large scan ranges. The combination of adaptable scan patterns with tomoSTED in particular is a very promising approach in order to combine the advantages of both techniques, however, experimental improvements are necessary to explore its full potential. TomoSTED microscopy is so far limited not only by the speed of the scanning, but also by the speed of the pattern rotation.

Since adaptive scanning is identified as a promising approach for enhancing the performance of (tomo)STED microscopy, this work is dedicated to developing photoefficient and fast illumination schemes by combining the concepts of adaptive scanning and ultrafast pattern generation for tomoSTED microscopy. An integral part is the design, development and characterization of a suitable experimental setup allowing high flexibility with respect to the scan system and the choice of the depletion pattern. It thus combines an adaptive scan system for large scan ranges with novel ultrafast switchable STED depletion patterns generated via conical diffraction.

In this way, tomoSTED microscopy, which has so far been demonstrated with a line-wise switching of pattern orientations [Krü17, due to low switching rates of the therefore employed Spatial Light Modulator (SLM), is implemented with a pixel-wise switching of the pattern orientation, thus meeting all requirements for the undistorted observation of fast-moving cellular components.

Furthermore, this work focuses on the first-time implementation of adaptive scanning to STED microscopy for large fields of view. By exploiting the advantages of electro-optic deflectors for a fast response and a galvanometer scanner for a large scan range, FastRESCue for STED microscopy as a novel variant of RESCue is introduced. As RESCue, it allows imaging at a reduced light dose without compromising on the resolution, but additionally yields a significant reduction of the total acquisition time. 
For FastRESCue, the reduction of the total acquisition time is achieved by reducing the individual pixel dwell times, avoiding any modification of the scan field as is required for e.g. MINFIELD. Nonetheless, the way of data recording for a typical image scan is often highly inefficient: For the scan of a predefined area, it has to be ensured that the structure under investigation is enclosed by that area. Consequently, the recorded area is usually much larger than strictly necessary, where disproportions of 1/100 or higher are common. Attempts to limit the recorded area and thus the necessary recording time by first capturing a rudimentary image of the sample with a lower resolution and then re-recording selected regions of the pre-scan with high resolution are associated with a considerable additional effort. Moreover, they are difficult to apply for samples that are subject to changes, such as living cells or tissue, where components like filaments or vesicles move within the typical time of an image acquisition even when imaging is sped up, e.g. by reducing the field of view: For a movement with a speed of some $\mathrm{nm} / \mathrm{ms}$, as shown in $\mathrm{WRL}^{+} 08$, a displacement in the range of $\mu \mathrm{m}$ is expected for typical fields of view between consecutive acquisitions of the same region. The direct restriction of the scan region to e.g. a band around the structure of interest is the only efficient way of reducing the total acquisition time without compromising on the biological insight.

By a combination of both fast-switching depletion patterns as well as adaptive scanning, tomoSTED microscopy as a low-illumination imaging technique can be extended to its applications beyond sub-resolved imaging: The information of the 1D STED images recorded in the course of a tomoSTED acquisition has so far been only utilized for the reconstruction of a high-resolution image [Krü17. However, direct information about the sample is already directly present in the raw 1D images and can therefore be immediately exploited. Considering for example a filamentous structure, the application of only a single 1D depletion pattern of matching direction is perfectly reasonable, since a higher resolution increase in the direction of the structure's orientation is not yielding additional information. On the other hand, this implies that, from a scan with various $1 \mathrm{D}$ depletion patterns, information about the structure's orientation can be obtained without a previous reconstruction step. This paves the way for structure tracing algorithms which do not rely on an analysis of the structure's previous orientation, but can determine the current direction directly by a single pixel scan. For the realization of such a tracing algorithm, a pixel-wise switching of the pattern orientation with a high switching rate is of primary importance.

In the course of this work, adaptive 2D scanning methods for STED microscopy are developed, presented and evaluated for specific applications. They have the potential to improve the performance of STED microscopy with the aim of reducing the light dose and the total acquisition time at uncompromised resolution. These novel adaptive scanning methods pave the way for a complete new level of biological applications for targeted read-out high-resolution microscopy techniques. 


\section{Theoretical Framework}

For a suitable choice of an experimental realization of adaptive scanning methods as well as for an interpretation of experimental results, it is inevitable to understand the basic concepts of all underlying techniques. This includes STED microscopy, in particular tomoSTED microscopy, conical diffraction for the generation of depletion patterns as well as the electro-optic effect, which is exploited both for polarization control and as integral part of the novel scan system. All these concepts are introduced in the following.

The first part of this chapter gives an overview of STED microscopy (see section 2.1), focusing on the advantages of tomoSTED microscopy (see section 2.2 compared to the classical 2D STED implementation, which employs a helical phase mask and yields a two-dimensional resolution increase. First, the resolution enhancement along one direction is compared for both methods. Subsequently, the assembly of a two-dimensionally super-resolved image is discussed. Following a short introduction on sample-conserving imaging techniques in section 2.3 , the remaining part of this chapter is dedicated to the investigation of light interaction with crystalline materials (see section 2.4), including the phenomenon of conical diffraction and its potential for intensity pattern shaping as well as an elucidation of the electro-optic effect and its variable applications for both beam scanning and polarization control.

\subsection{Introduction to STED microscopy}

\subsubsection{Abbe's diffraction limit}

Fluorescence is the preferred contrast for the vast majority of microscopy investigations in the life sciences [Hel07] due to its non-invasivity and the possibility of structure-specific imaging via fluorescence labeling. In far-field imaging techniques such as fluorescence microscopy, the imaging process is based on focusing a propagating beam of light by means of lenses. Due to the wave-nature of light, the lens system causes the light to interfere, yielding an intensity pattern with a main diffraction maximum, which is commonly referred to as the focal spot [Hel07]. The spatial extent of this diffraction-limited focal spot determines whether sample features can be discerned and thus governs the achievable resolution of the microscope HBZ09.

For a mathematical description, the image of a point-like emitter, which is back-projected to the sample plane, is defined as the Point Spread Function (PSF) $h(x, y)$ HBZ09, Goo05. Here, $x, y$ denote the coordinates in the sample plane with $(x, y)=(0,0)$ being the geometric focus. The object's function $O(x, y)$ depicts the light-emitting object, e.g. the fluorophore distribution in a sample. In case of a space-invariant PSF and for incoherent image formation, its back-projected image $I(x, y)$ is hence given by the convolution of the object's function $O(x, y)$ 
with the PSF of the imaging system $h(x, y)$, yielding [Goo05]

$$
I(x, y)=(O * h)(x, y) .
$$

As first stated by Abbe [Abb73] and therefore regarded as Abbe's diffraction limit, the minimal distance of two objects in order to be discernible is given by

$$
\Delta \approx \frac{\lambda_{0}}{2 \mathrm{NA}}
$$

with $\lambda_{0}$ denoting the vacuum wavelength of the propagating light. The numerical aperture NA of the objective lens is given by

$$
\mathrm{NA}=n \sin \alpha
$$

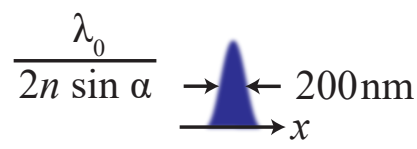

with $n$ being the refractive index of the immersion medium and $\alpha$ the half aperture angle of the lens. $\Delta$ is also the Full Width at Half Maximum (FWHM, $\Delta$ ) of the PSF $h(x, y)$ in the lateral direction [HSE09]. Due to the incomplete collection of the spherical wavefront by a single objective lens, the axial extent of the focal spot is significantly larger than the lateral. It is limited to HSE09.

$$
\Delta_{z} \approx \frac{n \lambda_{0}}{\mathrm{NA}^{2}}
$$

as illustrated in figure 2.1. The extent of the PSF for visible

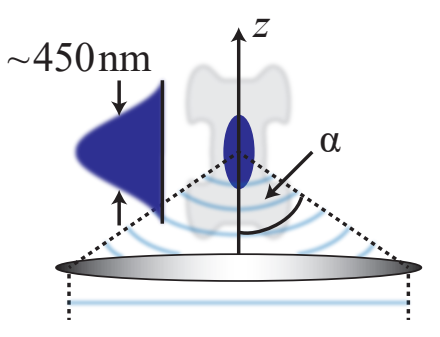

Figure 2.1: Illustration of the intensity distribution in the $x-z$ plane which is generated when a lens of half-aperture angle $\alpha$ focuses a laser beam (modified in accordance to [Hel07]).

light in the lateral direction, exploiting high NA objective lenses, is thus limited to approximately $200 \mathrm{~nm}$, whereas the axial extent is given by approximately $450 \mathrm{~nm}$ [Hel07.

When illuminating a fluorescently labeled object, all fluorescent markers within the spatial extent of the excitation PSF $h_{\text {exc }}(x, y)$ are excited simultaneously and thus emit contemporaneously within the pixel dwell time. Hence, details closer together than the diffraction limit cannot be discerned. For image acquisition, the emitted fluorescence is imaged onto a detector, which is again a process subject to diffraction. The detection PSF $h_{\text {det }}(x, y)$ describes the blurring of the position from which a photon is emitted [Hel07]. The effective PSF of an optical system is given by the product of the excitation and detection PSF as

$$
h(x, y)=h_{\mathrm{exc}}(x, y) \cdot h_{\mathrm{det}}(x, y) .
$$

Thus, the overall resolution of a microscope is affected by the FWHM of both the excitation and detection PSF. 
Several approaches have been pursued in order to improve the resolution, with confocal microscopy Min61 being among them. By employing a point-like illumination and detection, the lateral resolution can, compared to a wide-field microscope, theoretically be improved by a factor of $\sqrt{2}$, though this is practically compromised by the lower signal-to-noise ratio [WS84, Hel03]. Additionally, the out-of-focus fluorescence background signal is considerably reduced [HBZ09], making a confocal microscope an advantageous imaging technique in the life sciences. The PSF of a microscope can be approximated by a Gaussian in the central region of the PSF [ $\left.\mathrm{HKU}^{+} 08\right]$, which yields

$$
h_{\text {conf }}(x, y)=\frac{1}{2 \pi \sigma_{\text {conf }}^{2}} e^{-\frac{1}{2} \frac{x^{2}+y^{2}}{\sigma_{\text {conf }}^{2}}}
$$

for a confocal microscope with $\sigma_{\text {conf }}$ denoting the standard deviation. The latter is related to the FWHM $\Delta_{\text {conf via }}$

$$
\Delta_{\text {conf }}=2 \sqrt{2 \ln 2} \cdot \sigma_{\text {conf }}
$$

The prefactor in equation (2.1) is determined such that the PSF is normalized, i.e.

$$
\iint h_{\operatorname{conf}}(x, y) \mathrm{d} x \mathrm{~d} y=1
$$

Apart from confocal microscopy, several techniques have been developed aiming on improving the resolution, most of them via enhancing the objective's aperture and thus reducing the spot size. Both 4Pi microscopy [HS92] and ${ }^{5} \mathrm{M}$ (Image Interference Microscopy with Independent Incoherent Illumination, GAS99]) tackle the axial resolution, providing an up to sevenfold improvement over standard confocal microscopy. On the other hand, techniques like ISM (Image Scanning Microscopy, [ME10]) or SIM (Structured Illumination Microscopy, Gus00]), relying on the reassignment of fluorescence signal, have improved the lateral resolution by a factor of 2. Combining these techniques, as e.g. in $\mathrm{I}^{5} \mathrm{~S}$ as a combination of $\mathrm{I}^{5} \mathrm{M}$ and SIM, yields an isotropic resolution of around $100 \mathrm{~nm}\left[\mathrm{SIU}^{+} 08\right]$. Nevertheless, all these techniques are still fundamentally limited by diffraction.

Much higher image resolution seems only accessible by surpassing the diffraction barrier. One of the earliest approaches is near-field microscopy [PDL84, where diffraction is evaded by restricting the propagation distance of the light. Inherent to this technique is the short imaging depth [HBZ09], allowing near-surface observations only. For far-field observations, novel superresolution techniques based on exploiting the fluorophores' photophysics have been developed in the past decades. They improved fluorescence light microscopy to theoretically unlimited resolution [Hel07]. Both stochastic techniques, like PALM (PhotoActivatable Localization Microscopy, [BPS ${ }^{+}$06]) and STORM (STochastic Optical Reconstruction Microscopy, [RBZ06]), 
as well as techniques based on targeted read-out, as e.g. STED (STimulated Emission Depletion microscopy, [HW94]), GSD (Ground State Depletion, [HK95]) and RESOLFT (REversible Saturable Optically Linear Fluorescence Transitions, HEJH05]) microscopy, have proven to yield a resolution of down to $20 \mathrm{~nm}$ [Hel07]. In the following, STED microscopy as a super-resolution technique is described in more detail.

\subsubsection{Breaking the diffraction limit}

In all fluorescence microscopy techniques mentioned beforehand, molecular transitions between different energy levels, i.e. different states of the molecule, are of utter importance. For example in STED microscopy, a bright, electronically excited singlet state $S_{1}$ is employed together with the dark electronic ground state $S_{0}$ as illustrated in the Jablonski diagram in figure 2.2(a). Both states are expanded by higher vibronic levels, $S_{0 \text {,vib }}$ and $S_{1 \text {,vib }}$ respectively. The triplet state $T_{1}$ with its vibronic levels is included for the sake of completeness, though transitions to this state via intersystem crossing or phosphorescence back to the ground state $S_{0}$ are not considered in the following.

By the absorption of energy, fluorophores can alter their electronic, vibrational and rotational state [LC05]. The necessary energy for moving an electron to a different orbital, i.e. transferring the molecule from the dark ground state $S_{0}$ to the lowest energy level of the bright excited state $S_{1}$, can be induced by a photon of matching energy and thus wavelength. It is hereafter assumed that the fluorophore has been in the lowest level of $S_{0}$ before excitation,
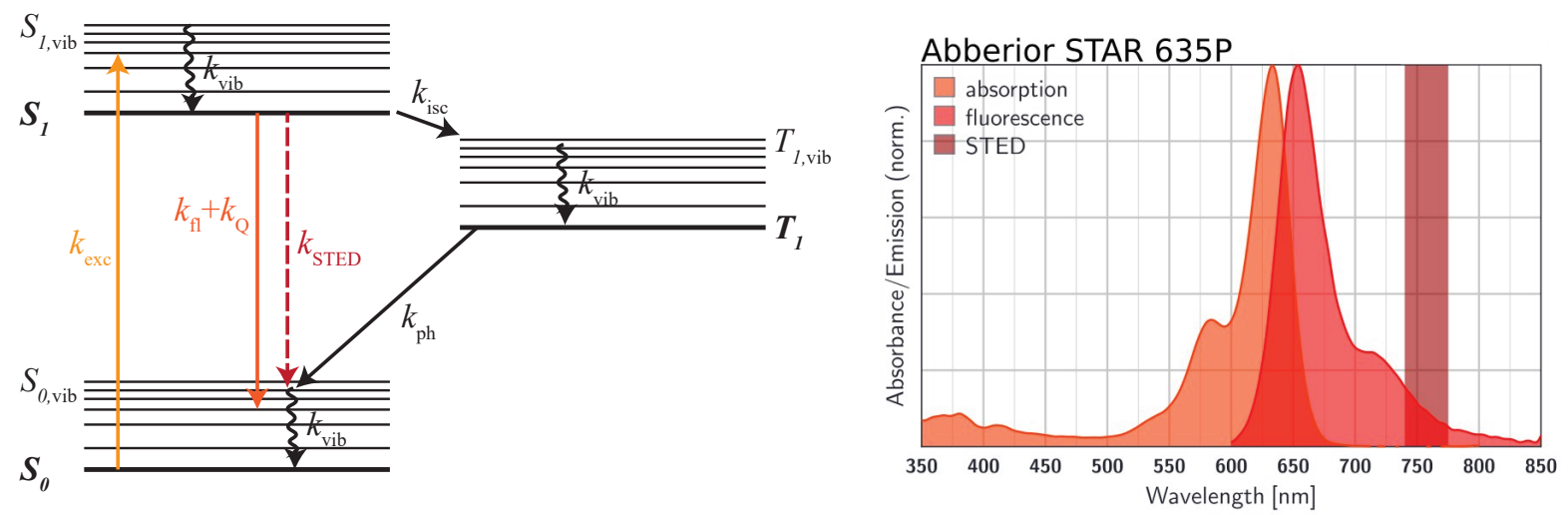

Figure 2.2: (a) The Jablonski diagram illustrates molecular transitions of an organic fluorophore. Transition paths between different energy levels are indicated by arrows with the according transition rates. Vibrational relaxations as indicated by curved arrows allow the non-radiating relaxation to the lowest energy level in the corresponding state (modified in accordance to [Hel07]). (b) Exemplary absorption und fluorescence spectrum for the dye Abberior STAR 635P (Abberior GmbH, Germany), clearly showing the Stokes shift as the red-shift of the fluorescence spectrum. The STED wavelength regime is, as indicated, at the far end of the fluorescence spectrum in order to allow spectral separation of stimulated and spontaneous emission as well as to avoid re-excitation by the STED laser (taken from Abb19). 
as it is the case for most fluorophores at room temperature [NC09. For incoming photons of shorter wavelength, i.e. higher deposited energies, the molecule can additionally undergo vibrational changes, ending up in one of the vibronic levels of $S_{1}$. Thus, the absorption process is characterized by a broad spectrum as indicated in figure 2.2(b), showing a sharper cut-off on its long-wavelength side [LC05].

For a molecule in the excited state there are different pathways leading back to the ground state. Internal conversion and vibrational relaxation, both radiation-free transitions, usually transfer the molecule from any singlet excited state to the lowest energy level of $S_{1}$ in the order of picoseconds [OP99, Val01. Due to a high energy gap between ground state and excited state in most fluorophores, the dominating way back to $S_{0}$ is the spontaneous emission of a photon, a process known as fluorescence [LC05]. The energy of the photon covers the gap between the lowest level of $S_{1}$ and any vibronic level of $S_{0}$ [LC05, yielding a fluorescence spectrum independent of the excitation wavelength as shown in figure 2.2(b). As some energy is transferred non-radiatively in the transition from a higher vibronic to the lowest level of $S_{1}$, the fluorescence spectrum is red-shifted compared to the excitation spectrum. This so-called Stokes shift [LC05] is the key to fluorescence microscopy, since it allows the separation of exciting and emitted light.

The time that a molecule stays in the excited state before returning to the ground state via spontaneous emission, known as the fluorescence lifetime $\tau_{\mathrm{fl}}$, is a crucial parameter for STED microscopy. With $\tau_{\mathrm{f}}$ typically being in the range of nanoseconds, it is much longer than the transition time to the excited state being in the range of femtoseconds [LC05] and is related to the fluorescence rate $k_{\mathrm{fl}}$ via

$$
k_{\mathrm{fl}}=\tau_{\mathrm{fl}}^{-1} .
$$

For two simultaneously emitting fluorophores with a distance below the diffraction limit, a spatial separation by means of conventional imaging is not possible. In order to discern such fluorophores, their emission needs to be separated in time. The concept of STED microscopy as well as many other super-resolution techniques therefore relies on the targeted switching of fluorophores, ensuring only specific fluorophores are in the bright state and thus potentially emitting at a specific time. In STED microscopy, this is realized by switching off all fluorophores not being at a pre-defined position $r_{i}$ [Hel07]. The process employed for the switching is stimulated emission, which is eponymous for STimulated Emission Depletion (STED) microscopy. In stimulated emission, a transition of the excited fluorophore from $S_{1}$ to $S_{0}$ is induced by an incident photon with a wavelength matching the energy gap between the lowest level of $S_{1}$ and any level of $S_{0}$. Simultaneously, a photon of the exact same properties as the incident photon is emitted [ST07]. Thus, by irradiating the fluorophore with a suitable light source, the excited state $S_{1}$ can be systematically depopulated. For effectively suppressing spontaneous emission, i.e. the fluorescent signal, this process needs to be conducted on a timescale shorter 
than the fluorescence lifetime [HW94. The wavelength chosen for stimulated emission depends on the absorption and fluorescence spectrum in order to prevent re-excitation by the STED light [Hel03] as well as to allow for a separation of fluorescent light and photons emitted due to stimulated emission. This typically leads to the choice of a red-shifted wavelength compared to absorption and fluorescence spectrum as illustrated in figure 2.2

For an efficient depletion process, pulsed lasers are employed for both excitation and STED beams [HW94]. The pulse duration of the STED pulse $\tau_{\text {STED }}$ is chosen in the range of some hundreds of picoseconds, ensuring that it acts within a fraction of the fluorescence lifetime [DKH05]. Since the vibrational relaxation takes place on a timescale being orders of magnitude smaller, $\tau_{\text {STED }}$ can be regarded as the smallest temporal unit [DKH05]. Hence, the total photon fluence $J_{\mathrm{STED}}$, measured in photons per area per pulse, is considered in the following, not dealing with the temporal shape of the STED pulse.

As for fluorescence, stimulated emission is governed by a rate $k_{\text {STED }}$ denoting the transitions per pulse, given by [DKH05]

$$
k_{\mathrm{STED}}=\gamma_{\mathrm{STED}} J_{\mathrm{STED}}
$$

depending on the pulse fluence $J_{\mathrm{STED}}$ as well as on the optical cross-section $\gamma_{\mathrm{STED}}$. The latter depicts the likelihood that the fluorophore interacts with the STED photon and thus depends on the fluorophore employed. In STED microscopy, the pulse fluence is a function of the position, as specially shaped depletion pattern distributions are chosen for the STED light focus. The superposition of this depletion pattern with a Gaussian excitation spot allows the inhibition of fluorescence wherever the depletion pattern's intensity and thus its pulse fluence is sufficiently high, resulting in a shrinking of the effective volume in which fluorescence is allowed.

The resulting resolution depends on the expected remaining fluorescence in presence of the inhibition light. This is defined as the suppression factor $\eta$ [DKH05, which can be well approximated by an exponential $\left.\mathrm{HKU}^{+} 08\right]$

$$
\eta(x, y)=e^{-\gamma_{\mathrm{STED}} J_{\mathrm{STED}}(x, y)}
$$

The suppression factor varies in space, as does the pulse fluence, and yields the normalized probability that a fluorophore remains in $S_{1}$ after the STED pulse has passed [DKH05]. Its non-linearity is the key to overcoming the diffraction limit [DKH05] and directly influences the expected resolution: The effective PSF of a STED microscope is given by the product of the confocal PSF with the suppression factor $\left[\mathrm{HKU}^{+} 08\right]$

$$
h_{\mathrm{eff}}(x, y)=h_{\mathrm{conf}}(x, y) \cdot \eta(x, y) .
$$


The pulse fluence as the free parameter for changing the resolution enhancement is usually not directly accessible in experiments and hence a description of the pulse fluence is sought for. The pulse fluence is described by the overall number of photons per pulse $N_{\text {STED }}$ and the STED light distribution as Krü17]

$$
J_{\mathrm{STED}}(x, y)=N_{\mathrm{STED}} \cdot h_{\mathrm{STED}}(x, y) .
$$

Here, the PSF of the STED depletion pattern $h_{\text {STED }}$ denotes the focal probability distribution of a single photon of the inhibition light and is normalized, i.e.

$$
\iint h_{\mathrm{STED}} \mathrm{d} x \mathrm{~d} y=1
$$

Furthermore, $N_{\mathrm{STED}}$ is related to the experimentally accessible overall STED laser power $P_{\mathrm{STED}}$ in the back focal plane of the objective via

$$
N_{\mathrm{STED}}=\frac{P_{\mathrm{STED}}}{k_{\mathrm{rep}} \cdot \frac{h_{P} \cdot c}{\lambda_{0, \mathrm{STED}}}}
$$

where $h_{P}$ stands for the Planck constant, $c$ for the speed of light in vacuum and $\lambda_{0, \mathrm{STED}}$ for the wavelength of the light employed.

To characterize the depletion strength, it is convenient to introduce the saturation fluence $J_{\text {sat }}$, which is defined as the laser fluence at which fluorescence is suppressed by a factor of 2 . Following equation $(2.2)$, it is given by

$$
J_{\mathrm{sat}}=\frac{\ln 2}{\gamma_{\mathrm{STED}}}
$$

and is hence not spatially varying. Analogously to $J_{\mathrm{STED}}$, it can be related to the saturation laser power $P_{\text {sat }}$ via Krü17.

$$
N_{\mathrm{sat}}=J_{\mathrm{sat}} \cdot A
$$

where $A$ determines the area of the focal spot and $N_{\text {sat }}$ is the associated overall number of photons per pulse, given by

$$
N_{\mathrm{sat}}=\frac{P_{\mathrm{sat}}}{k_{\mathrm{rep}} \cdot \frac{h_{P} \cdot c}{\lambda_{0, \mathrm{STED}}}} .
$$

Here, $P_{\text {sat }}$ denotes the experimentally tangible saturation laser power in the back focal plane of the objective. Since the area of the focal spot $A$ is identical for all STED variants considered within this thesis, it is without loss of generality assumed to be equal to one. $N_{\text {sat }}$ includes not only information about the dye properties like the fluorescence lifetime $\tau_{\mathrm{fl}}$ or the optical cross-section $\gamma_{\mathrm{STED}}$, but is also influenced by the laser characteristics, namely the wavelength 
$\lambda_{0, \text { STED }}$ and the repetition rate $k_{\text {rep }}$. A quantity independent of i.a. the laser's repetition rate is the so-called saturation factor $\zeta$, defined as [Krü17]

$$
\zeta=\frac{N_{\mathrm{STED}}}{N_{\mathrm{sat}}}=\frac{P_{\mathrm{STED}}}{P_{\mathrm{sat}}},
$$

which will be used as the unit of laser power for all further considerations. Substituting equations (2.4), (2.2), 2.1) and (2.5) in (2.3) yields

$$
h_{\mathrm{eff}}(x, y)=\frac{1}{2 \pi \sigma_{\mathrm{conf}}^{2}} e^{-\frac{1}{2} \frac{x^{2}+y^{2}}{\sigma_{\mathrm{conf}}^{2}}-\ln 2 \cdot \zeta \cdot h_{\mathrm{STED}}(x, y)} .
$$

For a detailed analysis of the resolution enhancement in STED microscopy, the knowledge of the depletion PSF $h_{\text {STED }}$ is inevitable. This is subject to the following section, yielding a comparison between the classical two-dimensional implementation via a doughnut-shaped depletion pattern and an implementation providing only a one-dimensional resolution enhancement.

\subsection{Tomographic STED microscopy}

In the previous section, the effective PSF's dependence on the applied laser power as well as on the depletion pattern's shape was deduced. In the following, the potential of a depletion pattern providing a one-dimensional resolution increase is evaluated. Since such a pattern has to be rotated in order to obtain an image with an isotropic resolution enhancement, there are two fundamental aspects to be addressed: First, the resolution increase along one axis for the classical 2D and the novel 1D implementation is compared. Subsequently, the transfer of highresolution information into a reconstructed image is discussed and analyzed in dependence on the number of pattern orientations.

\subsubsection{Resolution increase along one direction}

According to equation (2.6), the resulting effective PSF and thus the expected resolution increase strongly depends on the depletion pattern distribution $h_{\mathrm{STED}}$, which is therefore analyzed in the following. As depicted in figure 2.3(b), the classical 2D depletion pattern is doughnutshaped. It is generated by the helical phase plate shown in figure 2.3. (a) for incident light of right-handed circular polarization. Mirror-symmetrical points with respect to the optical axis of the beam experience a phase shift of $\pi$, causing the $x$ - and $y$-components to interfere destructively when focused by the objective lens. The $z$-component cancels out when additionally considering a second point pair rotated by $90^{\circ}$ with respect to the first one, yielding the central zero of the intensity distribution.

For a $1 \mathrm{D}$ resolution increase, a $0 / \pi$ phase step as indicated in figure 2.3 (c) is sufficient. The illumination with linearly polarized light with polarization direction perpendicular to the direction of the phase step leads to destructive interference along a line when focused by the 
objective lens. The resultant depletion pattern for an exemplary resolution increase along the $x$-axis is illustrated in figure 2.3(d) and furthermore referred to as 1D depletion pattern.

For a pattern comparison, profiles along the high-resolution axis are compared and depicted in figure 2.3(e) for both the 2D (black) and the 1D (red) pattern. To determine the expected resolution increase, $h_{\mathrm{STED}}$ is approximated by a parabola in the vicinity of the geometric focus $(x, y)=(0,0)$, i.e. close to the central minimum in figure 2.3(e). This yields [Krü17]

$$
h_{\mathrm{STED}, 2 \mathrm{D}}(x, y) \approx \frac{1}{2 \ln 2} a_{2 \mathrm{D}}\left(x^{2}+y^{2}\right)
$$

for the approximation of the $2 \mathrm{D}$ profile with a two-dimensional parabola and

$$
h_{\mathrm{STED}, 1 \mathrm{D}}(x, y) \approx \frac{1}{2 \ln 2} a_{1 \mathrm{D}} x^{2}
$$

for the one-dimensional parabola. Here, a 1D depletion pattern with high-resolution axis along the $x$-axis is assumed. $a_{2 \mathrm{D}}$ and $a_{1 \mathrm{D}}$ denote the respective pattern steepness, which is proportional to the second derivative of the STED PSFs for $y=0$ evaluated at $x=0$. The functional behavior of the second derivative for both the $1 \mathrm{D}$ and the $2 \mathrm{D}$ depletion pattern is

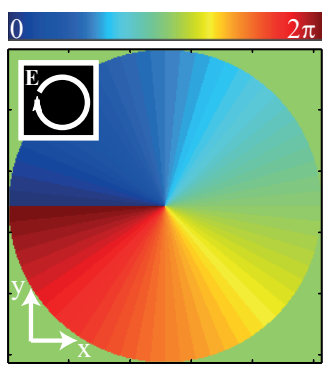

(a)

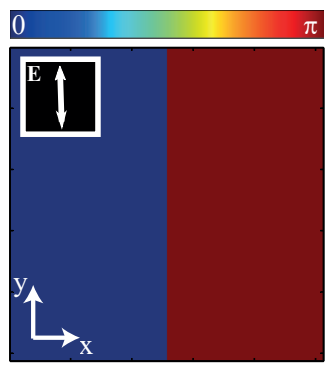

(c)

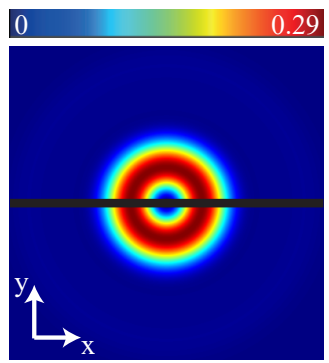

(b)

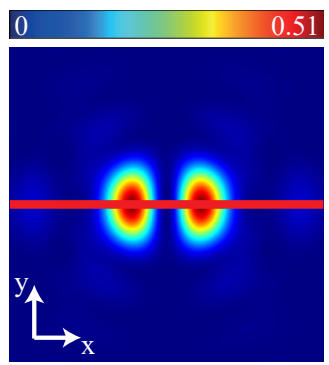

(d)

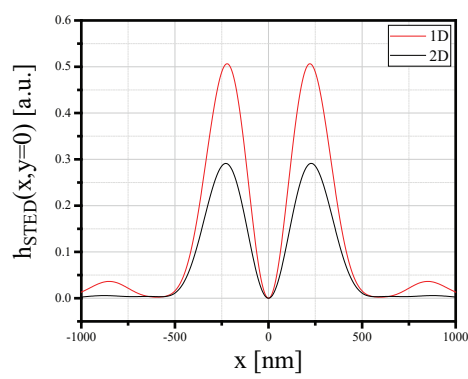

(e)

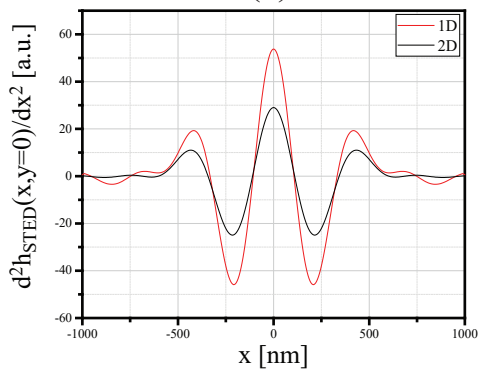

(f)

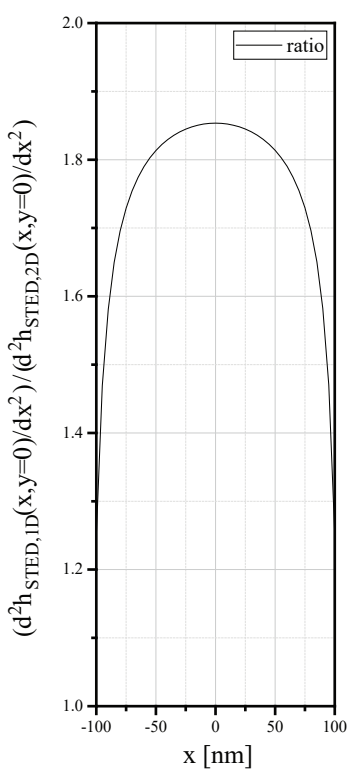

(g)

Figure 2.3: Simulation of $2 \mathrm{D}$ and $1 \mathrm{D}$ depletion patterns. (a) Helical phase mask with corresponding polarization for generation of (b) the doughnut-shaped depletion pattern employed for 2D STED microscopy. An alternative depletion pattern, resulting in a one-dimensional resolution enhancement is generated by (c) a $0 / \pi$ phase plate with corresponding linear polarization resulting in (d). (e) A comparison of intensity profiles for the two patterns as well as (f) their second derivatives yields a 1.85 higher pattern steepness at the pattern's intensity zero for the 1D pattern compared to the 2D pattern, as can be seen from the ratio in (g) (modified in accordance to [Krü17]). 
shown in figure 2.3(f), yielding a higher value for the 1D pattern in the vicinity of the central minimum. For a quantitative analysis, the ratio of these curves is calculated (see figure 2.3 (g)), indicating a higher pattern steepness for the 1D depletion pattern with

$$
\frac{a_{1 \mathrm{D}}}{a_{2 \mathrm{D}}} \approx 1.85
$$

The effect of the pattern steepness on the obtainable resolution enhancement is evaluated by substituting expressions (2.7) and (2.8) in equation (2.6), yielding an effective PSF of

$$
\begin{aligned}
& h_{\mathrm{eff}, 2 \mathrm{D}}(x, y)=\frac{1}{2 \pi \sigma_{\mathrm{conf}}^{2}} e^{-\frac{1}{2} \frac{x^{2}+y^{2}}{\sigma_{\mathrm{conf}}^{2}}\left(1+\sigma_{\mathrm{conf}}^{2} \cdot \zeta \cdot a_{2 \mathrm{D}}\right)}, \\
& h_{\text {eff, } 1 \mathrm{D}}(x, y)=\frac{1}{2 \pi \sigma_{\text {conf }}^{2}} e^{-\frac{1}{2} \frac{x^{2}}{\sigma_{\text {conf }}^{2}}\left(1+\sigma_{\text {conf }}^{2} \zeta \cdot a_{1 \mathrm{D}}\right)-\frac{1}{2} \frac{y^{2}}{\sigma_{\text {conf }}^{2}}}
\end{aligned}
$$

for 2D and 1D depletion pattern with a resulting standard deviation of [Krü17]

$$
\sigma_{\mathrm{STED}, 1 \mathrm{D} / 2 \mathrm{D}}=\sigma_{\mathrm{conf}} \frac{1}{\sqrt{1+\sigma_{\mathrm{conf}}^{2} \cdot a_{1 \mathrm{D} / 2 \mathrm{D}} \cdot \zeta}}
$$

along the high-resolution axis. The resolution increase is defined as

$$
k=\frac{\sigma_{\mathrm{conf}}}{\sigma_{\mathrm{STED}}}=\frac{\Delta_{\mathrm{conf}}}{\Delta_{\mathrm{STED}}}
$$

with the FWHM of the STED image given by

$$
\Delta_{\mathrm{STED}}=2 \sqrt{2 \ln 2} \cdot \sigma_{\mathrm{STED}}
$$

Following equation (2.11), differences in the resolution increase for the same STED laser power, i.e. the same saturation factor $\zeta$, between the two methods are directly related to differences of the pattern steepness $a$. The pattern steepness includes geometrical aspects like the numerical aperture as well as information about the phase modulation [Krü17]. Assuming a STED laser power well above the saturation level, the resolution increase for same STED laser power compares according to

$$
k_{1 \mathrm{D}} \approx \sqrt{1.85} \cdot k_{2 \mathrm{D}} \approx 1.36 \cdot k_{2 \mathrm{D}}
$$

On the other hand, the same resolution increase $k_{1 \mathrm{D}}=k_{2 \mathrm{D}}$, i.e. $\sigma_{\mathrm{STED}, 1 \mathrm{D}}=\sigma_{\mathrm{STED}, 2 \mathrm{D}}$, along the high-resolution axis is expected for a saturation factor given by

$$
\zeta_{1 \mathrm{D}}=\frac{\zeta_{2 \mathrm{D}}}{1.85}
$$


This implies that for achieving the same resolution increase along one direction, only approximately half of the STED power is needed for the 1D depletion pattern compared to the classical 2D pattern.

Apart from the resolution increase, the signal transfer's dependence on the spatial frequency plays a crucial role for the resulting image quality and is used to characterize the performance of an optical system [Krü17]. It can be quantified by the Optical Transfer Function (OTF), which is defined as the spatial Fourier transform of the PSF.

For simplifying the further considerations, equations 2.9 and 2.10 can be generalized by Krü17

$$
h_{\mathrm{eff}}(x, y)=\frac{1}{2 \pi \sigma_{\mathrm{conf}}^{2}} e^{-\frac{1}{2}\left(\frac{x^{2}}{\sigma_{x}^{2}}+\frac{y^{2}}{\sigma_{y}^{2}}\right)}
$$

with $\sigma_{x}=\sigma_{y}=\sigma_{\mathrm{STED}}$ for the $2 \mathrm{D}$ pattern and $\sigma_{x}=\sigma_{\mathrm{STED}}, \sigma_{y}=\sigma_{\mathrm{conf}}$ for the $1 \mathrm{D}$ pattern, assuming the same resolution increase

$$
k=k_{1 \mathrm{D}}=k_{2 \mathrm{D}}=\frac{\sigma_{\mathrm{conf}}}{\sigma_{\mathrm{STED}}}
$$

for both methods. Hitherto, only profiles of $h_{\mathrm{eff}}$ along one direction have been considered. For an evaluation of the signal transfer's dependence on the spatial frequency, the two-dimensional PSF is taken into consideration. The resulting OTF is given by Krü17.

$$
\operatorname{OTF}(u, v)=e^{-2 \pi^{2}\left(u^{2} \sigma_{x}^{2}+v^{2} \sigma_{y}^{2}\right)} \cdot \begin{cases}\frac{1}{k} & \text { for the 1D pattern } \\ \frac{1}{k^{2}} & \text { for the 2D pattern }\end{cases}
$$

which can be transformed to polar coordinates $(f, \phi)=\left(u^{2}+v^{2}, \arctan \frac{u}{v}\right)$ resulting in

$$
\operatorname{OTF}(f, \phi)=e^{-2 \pi^{2}\left(\sin ^{2} \phi \sigma_{x}^{2}+\cos ^{2} \phi \sigma_{y}^{2}\right) f^{2}} \cdot \begin{cases}\frac{1}{k} & \text { for the 1D pattern } \\ \frac{1}{k^{2}} & \text { for the 2D pattern }\end{cases}
$$

The resultant overall fluorescence signal, assuming a signal of 1 in absence of the STED beam, is given by the central value of the OTF, i.e.

$$
\mathrm{OTF}_{2 \mathrm{D}}(0,0)=\frac{1}{k^{2}}
$$

for the 2D depletion pattern and analogously for the 1D pattern

$$
\mathrm{OTF}_{1 \mathrm{D}}(0,0)=\frac{1}{k} \text {. }
$$

Consequently, the signal transfer for same resolution increase is $k$ times higher for a $1 \mathrm{D}$ depletion pattern. 


\subsubsection{Isotropic resolution enhancement}

So far, only one pattern orientation for the 1D depletion pattern has been considered and shown to be beneficial in terms of resolution enhancement and signal transfer compared to the $2 \mathrm{D}$ depletion pattern. For a one-dimensional structure with suitably chosen pattern direction, these advantages can be fully exploited. However, most samples contain two-dimensional structures, and even filament-like networks demonstrate a variety of orientations, demanding an isotropic resolution increase in all lateral directions. This results in the necessity to rotate the 1D depletion pattern around an axis perpendicular to the focal plane, intersecting with the aforementioned in the focal center [Krü17]. A sequence of images, hereafter referred to as sub-images, is acquired with pattern orientations in the range from $0^{\circ}$ to $180^{\circ}$. Here, the angle is given between the $x$-axis and and the low-resolution axis. Subsequently, all sub-images are combined to yield a, in terms of resolution enhancement, rotationally symmetric reconstruction ([Krü17], see also section 3.4). This reconstructed image is in the following referred to as the tomoSTED image.

For the 2D pattern, the resolution increase along the two axes is the same, resulting in a rotationally symmetric OTF (cf. equation (2.12)

$$
\mathrm{OTF}_{2 \mathrm{D}}(f)=\frac{1}{k_{2 \mathrm{D}}^{2}} e^{-2 \pi^{2} \sigma_{\mathrm{STED}}^{2} f^{2}}
$$

In the following, two cases for the 2D STED OTF are considered and displayed in figure 2.4. firstly, the 2D STED OTF for same laser power (dark grey) as compared to the 1D case, secondly the 2D STED OTF for same resolution enhancement (light grey) at FWHM compared to the elliptical 1D STED OTF (green) for a resolution enhancement of $k_{1 \mathrm{D}}$. Due to the ellipticity of the 1D STED OTF, the challenge of the pattern rotation, from a theoretical point of view, is the determination of the minimal number of angles necessary to transfer the highresolution information from the sub-images into an artifact-free reconstruction.

The image quality obtainable in the reconstructed tomoSTED image strongly depends on the reconstruction procedure employed and particularly on the amplitude and isotropy of the assembled OTF. A first reconstruction approach, presented and evaluated in detail in [Krü17, is the maximum-value reconstruction, for which only the information along the high-resolution axis is kept from each sub-image. By reducing the elliptical OTF to its major axis in case of a continuous rotation of the depletion pattern, it is thus possible to fully transfer the resolution enhancement into the reconstructed image [Krü17]: The resulting tomoSTED OTF [Krü17.

$$
\mathrm{OTF}_{\text {tomo }}(f) \approx \frac{1}{k_{1 \mathrm{D}}} e^{-2 \pi^{2} \sigma_{\mathrm{STED}}^{2} f^{2}}
$$

is radially symmetric and differs from $\mathrm{OTF}_{2 \mathrm{D}}$ only by a scaling factor for same resolution enhancement $k=k_{1 \mathrm{D}}=k_{2 \mathrm{D}}$. 
However, for a legitimate comparison of 2D STED and tomoSTED microscopy, the total acquisition time has to be the same for both methods, resulting in a rescaling of the OTF: For same resolution enhancement $k$, implying a lower depletion light dose for tomoSTED microscopy, and $N$ sub-images acquired for different 1D depletion pattern orientations, the signal of each sub-image is rescaled by a factor of $1 / N$, yielding

$$
\mathrm{OTF}_{\text {tomo, scaled }}(f) \approx \frac{1}{k_{1 \mathrm{D}} \cdot N} e^{-2 \pi^{2} \sigma_{\mathrm{STED}}^{2} f^{2}}
$$

As elucidated in [Krü17, a continuous rotation, i.e. $N \rightarrow \infty$, is thus unfeasible in realistic imaging conditions due to a too low signal-to-noise ratio (SNR). The challenge is therefore to choose $N$ high enough to achieve an artifact-free, i.e. quasi-homogeneous reconstruction, but as low as possible to obtain a high SNR.

A compromise for the number of pattern orientations is given by covering the 2D STED OTF for same STED laser power, i.e. with radius $k_{2 \mathrm{D}}=\frac{k_{1 \mathrm{D}}}{1.36}$, with a sufficient number of elliptical 1D STED OTFs. In this way, a higher information transfer in tomoSTED compared to 2D

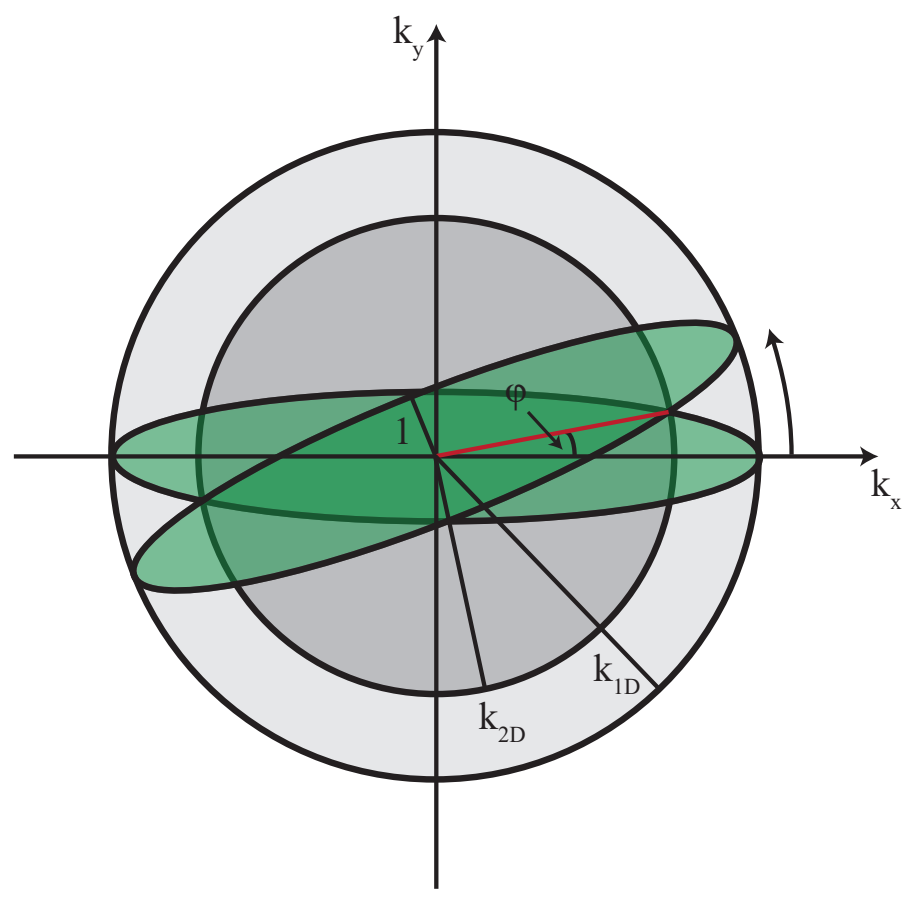

Figure 2.4: Visualization of the $1 \mathrm{D}$ and 2D STED OTFs in Fourier space. The OTF as given by equation 2.12 shows a Gaussian distribution. The circles and ellipses depicted here are the iso-lines at FWHM. The 1D STED OTF is shown in green with the confocal axis being normalized to a FWHM of 1. The FWHM of the high resolution axis is consequently given by $k_{1 \mathrm{D}}$ in the $1 \mathrm{D}$ case. The $2 \mathrm{D}$ STED OTF resulting from the same applied laser power as employed for the $1 \mathrm{D}$ case is shown in dark grey with a radius of $k_{2 \mathrm{D}}$, whereas the $2 \mathrm{D}$ STED OTF for same resolution enhancement is depicted in light grey. 
STED microscopy at same laser power is guaranteed. In order to determine the number of ellipses necessary, the intersection between the circular 2D STED OTF and the 1D STED OTF is calculated, yielding

$$
k_{x}^{2}=k_{1 \mathrm{D}}^{2} \frac{k_{2 \mathrm{D}}^{2}-1}{k_{1 \mathrm{D}}^{2}-1}, \quad k_{y}^{2}=\frac{k_{1 \mathrm{D}}^{2}-k_{2 \mathrm{D}}^{2}}{k_{1 \mathrm{D}}^{2}-1} .
$$

The resulting number of pattern orientations can be determined as

$$
N_{\mathrm{opt}, 1}=\frac{\pi}{2 \varphi}=\frac{\pi}{2 \arctan \sqrt{\frac{k_{1 \mathrm{D}}^{2}-k_{2 \mathrm{D}}^{2}}{k_{1 \mathrm{D}}^{2}\left(k_{2 \mathrm{D}}^{2}-1\right)}}}=\frac{\pi}{2 \arctan \sqrt{\frac{\beta^{2}-1}{k_{1 \mathrm{D}}^{2}-\beta^{2}}}}
$$

with $\varphi$ being the half-angle between two successive orientations of the 1D STED OTF and $k_{1 \mathrm{D}}=\beta \cdot k_{2 \mathrm{D}}$ being the scaling between the resolution enhancement for $1 \mathrm{D}$ and $2 \mathrm{D}$. For same STED laser power, $\beta \approx 1.36$ and thus

$$
N_{\mathrm{opt}, 1}=\frac{\pi}{2 \arctan \sqrt{\frac{0.85}{k_{1 \mathrm{D}}^{2}-1.85}}} \approx 1.085 \cdot \frac{\pi}{2} k_{1 \mathrm{D}} .
$$

where the last approximation holds for large $k_{1 \mathrm{D}}$.

An alternative approximation is given in Krü17 by the approach of covering the 2D STED

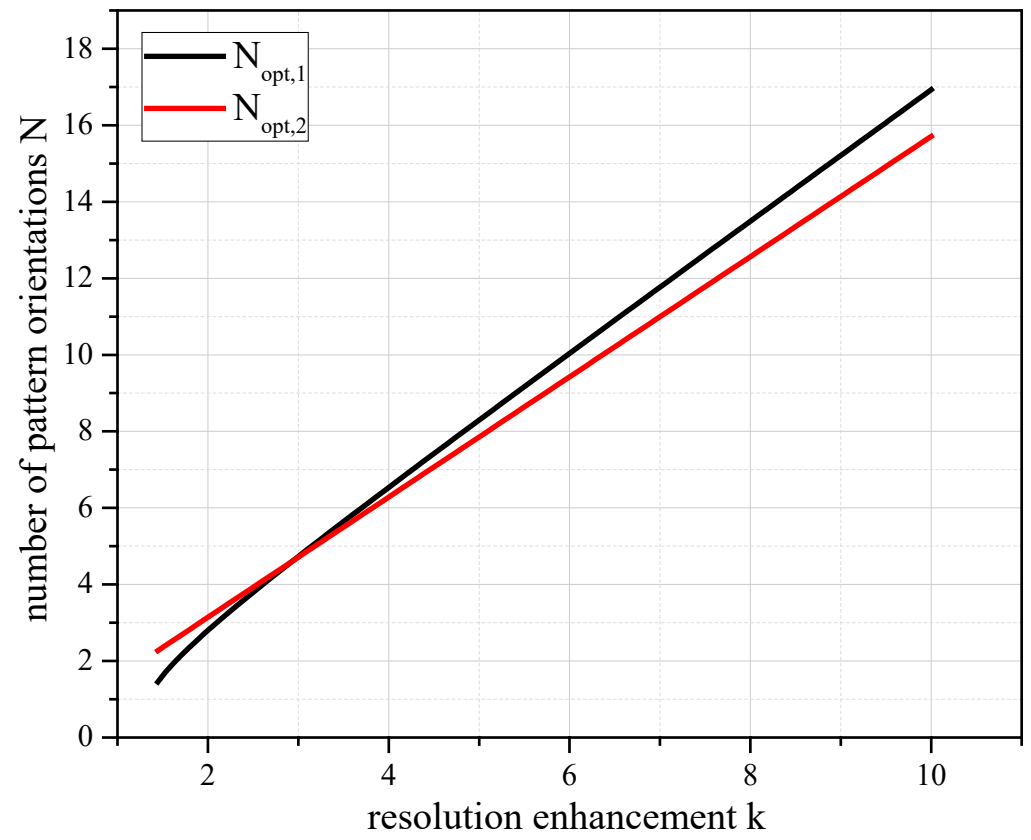

Figure 2.5: Comparison of the two criteria presented for the optimal number of pattern orientations. $N_{\text {opt }, 1}$, derived from the comparison with the 2D STED OTF at same STED laser power shows a higher slope than $N_{\mathrm{opt}, 2}$, introduced in [Krü17]. 
OTF for same resolution enhancement (light grey in figure 2.4) with the elliptical 1D STED OTFs by sampling the circumference of the circular 2D STED OTF by the minor axis of the 1D STED OTF. This leads to

$$
N_{\mathrm{opt}, 2} \approx \frac{\pi}{2} k_{1 \mathrm{D}}
$$

A comparison between $N_{\mathrm{opt}, 1}$ and $N_{\mathrm{opt}, 2}$ is displayed in figure 2.5. The difference between the two criteria for large $k$ is in the range of less than $9 \%$, with $N_{\text {opt,1 }}$ showing a higher slope than $N_{\mathrm{opt}, 2}$. Moreover, $N_{\mathrm{opt}, 1}>N_{\mathrm{opt}, 2}$ for $k>3$. Consequently, $N_{\mathrm{opt}, 1}$ suffices whenever $N_{\mathrm{opt}, 2}$ has been shown to yield artifact-free results.

Choosing $N_{\mathrm{opt}, 2}$ for tomoSTED microscopy has been shown to be sufficient for an artifactfree reconstruction [Krü17]. Indeed, when applying the Richardson-Lucy deconvolution (cf. section 3.4, the number of pattern orientations can be reduced to $N_{\mathrm{opt}, 2} / 2$ without compromising on the image quality, since $\mathrm{OTF}_{\text {tomo, scaled }}$ for $N_{\mathrm{opt}, 2}$ still outdoes $\mathrm{OTF}_{2 \mathrm{D}}$ at high spatial frequencies [Krü17. This enables imaging with tomoSTED microscopy with approximately one fourth of the depletion light dose compared to 2D STED microscopy for the same resolution enhancement and a comparable image quality.

\subsection{Sample-conserving imaging techniques}

Reducing the light dose imposed on the sample is crucial for any sub-diffraction imaging technique in order to avoid sample damage. As the principle of STED microscopy relies on a cycling of molecules between an 'on' and an 'off' state, avoiding the molecule's transition to a longlived dark state is of utter importance, since these molecules can no longer be excited during the remaining acquisition time. Moreover, irreversible photochemical reactions can occur for molecules in the triplet-state, leading to bleaching and phototoxicity [LC05]. An immediate approach to avoid the long-lived dark state is the use of antibleaching agents [ $\left.\mathrm{VKS}^{+} 08\right]$. Yet, they are typically incompatible with live-cell imaging and strongly depend on the dyes employed $\left[\mathrm{SER}^{+} 11\right]$.

For any structures separated by more than the diffraction limit, the application of e.g. a STED beam is avoidable. Wherever adjacent features need to be discerned, light of a certain threshold intensity, where typically the saturation intensity is taken as a measure, has to be applied for switching off the fluorophores. However, depending on the desired resolution, a pattern providing the required intensity close to the central minimum will inevitably show considerably higher intensities in the maximum, which are not necessary for the efficient switching of fluorophores.

Several techniques have recently been presented tackling these two aspects in order to avoid 
unnecessary state transitions. For the method named REduction of State transition Cycles (RESCue), a decision process based on the number of meanwhile detected photons is conducted during a fraction of the pixel dwell time [SER $\left.{ }^{+} 11\right]$. The beam exposure is interrupted when the desired signal to noise ratio is reached, i.e. enough photons are collected. In this case, the resultant signal is scaled respecting the actual acquisition time. Additionally, if there is not enough signal detected, i.e. in the absence of a fluorescent object at the actual scan position, the illumination of both excitation and suppression beam is stopped for the residual pixel dwell time [SER $\left.{ }^{+} 11\right]$. RESCue has shown to yield an up to eightfold bleaching reduction on biological samples, depending on the sample structure $\left[\mathrm{SER}^{+} 11\right]$.

RESCue avoids unnecessary illumination of the sample, e.g. if enough photons are detected, but does not tackle the aspect of the high intensity regions in the outer parts of the suppression beam's PSF. A technique called MINFIELD has been presented recently [GPH ${ }^{+} 17$ ], which avoids the sample's exposure with high intensities in the region of interest by restricting the scanning to a sub-diffraction-sized region. By only applying the innermost part of the intensity distribution on the structure of interest with the intensity maxima laying outside the scan region, over 100 times more signal than in comparable standard imaging conditions can be observed. Nevertheless, this implies the restriction to scan fields in the order of $100 \times 100 \mathrm{~nm}^{2}$, since the neighboring areas experience the high beam intensities.

A more dynamic approach is given by the method Dynamic Intensity Minimum (DyMIN), which generalizes the concepts of RESCue and MINFIELD [ $\left.\mathrm{HRH}^{+} 17\right]$. As in RESCue, a pixelbased decision is taken based on the probing of the sample. Additionally, the intensity of the suppression beam for probing and acquisition is dynamically adjusted, allowing to only use as much intensity as required. By adjusting the applied laser power, the resolution is dynamically tuned, governed by the density of the structure and thus the required separation. If the applied suppression power is large, the fluorophores are close to each other and thus close to the central minimum of the intensity distribution, implying that they do not experience the high powers in the rim. This takes in the full advantages of MINFIELD, but without limiting the field of view $\mathrm{HRH}^{+} 17$. The technique has been shown to lower the light dose up to 20-fold compared to classical STED microscopy under common biological imaging conditions and even more for sparse samples $\mathrm{HRH}^{+} 17$.

None of the beforehand mentioned methods is adaptive in time, although some of them show dead times during laser off-switching. A non-deterministic scanning approach for RESOLFT nanoscopy with reversible switchable fluorescent proteins has recently been demonstrated to decrease the acquisition time and lower the photodosage $\left[\overline{\mathrm{DCC}^{+} 19}\right]$. The smart RESOLFT principle uses the lower threshold for structure detection by probing the sample with a probing beam as also performed for RESCue. Contrary to the latter, the lasers are then not just switched off, but depending on the outcome, the scan position is moved forward. This results in an up to sixfold faster acquisition and a $70-90 \%$ reduction of the light dose. The concept of 
adjusting the pixel time and changing the scan sequence can be also generalized for the other here presented sample-preserving imaging techniques. An analogue to smart RESOLFT for STED microscopy is presented herein (see section 3.5).

\subsection{Light interaction with crystalline materials}

This section is dedicated to the formal description of light waves and their interaction with matter, in particular with crystalline materials. The first subsection introduces polarization states as well as a matrix formalism for describing their interaction with e.g. wave-plates. Subsequently, the effect of birefringence is discussed in general, with a following subsection on conical diffraction, a phenomenon occurring in biaxial crystals under specific conditions. This phenomenon is exploited for the depletion pattern generation in the course of this work as elucidated in section 3.2. Induced birefringence by the application of an electric field is elaborated afterwards, as it is employed for scanning (cf. section 3.5) as well as for polarization control (cf. section 3.3), followed by the final subsection on a specific case of photoelasticity, the acousto-optic effect, utilized for the fast on-off switching of the lasers.

\subsubsection{Description of polarization states}

In the following, it is assumed that monochromatic light is propagating in a homogeneous, nonconducting, isotropic medium. The coordinate system is chosen such that the propagation direction coincides with the $z$-axis. The polarization of light is generally defined by the electric field vector ST07.

$$
\boldsymbol{E}(z, t)=\Re\left\{\boldsymbol{E}_{0} e^{i \omega\left(t-\frac{z}{v_{p}}\right)}\right\}
$$

and hence varies with time. It is characterized by its complex envelope $\boldsymbol{E}_{0}$, determining the amplitude of the electric field, as well as by the angular frequency $\omega$ and phase velocity $v_{p}$. At each time point $t$ and position $z, \boldsymbol{E}(z, t)$ lies in a plane tangential to the wavefront. If the wavefront changes direction for different positions $z$, also the trace defined by the tip of the electric-field vector varies with the position. For simplicity, this is omitted in the following by considering only plane waves, for which the wavefronts are infinite parallel planes.

For a plane wave propagating in $z$-direction, the electric field oscillates in the $x$-y-plane and can thus be split in any two orthogonal components $E_{x}, E_{y}$ along the coordinate axes $\widehat{\boldsymbol{x}}, \widehat{\boldsymbol{y}}$, which fully characterize $\boldsymbol{E}$. They can be expressed as [ST07]

$$
E_{x}(z, t)=E_{0, x} \cos \left[\omega\left(t-\frac{z}{v_{p}}\right)+\varphi_{x}\right]
$$


and

$$
E_{y}(z, t)=E_{0, y} \cos \left[\omega\left(t-\frac{z}{v_{p}}\right)+\varphi_{y}\right]
$$

with the amplitude vector $\boldsymbol{E}_{0}$ given by

$$
\boldsymbol{E}_{0}=E_{0, x} e^{i \varphi_{x}} \widehat{\boldsymbol{x}}+E_{0, y} e^{i \varphi_{y}} \widehat{\boldsymbol{y}}
$$

Here, $E_{0, x}, \varphi_{x}, E_{0, y}$ and $\varphi_{y}$ denote the respective amplitudes and phases. Both components $E_{x}(z, t)$ and $E_{y}(z, t)$ vary sinusoidally with time with in general different amplitudes and phases. Thus, the endpoint of the electric field vector traces an ellipse, called the polarization ellipse. The course of the tip of the electric field vector at a fixed time $t$ is graphically displayed in figure 2.6(a). It follows a helical trajectory on the surface of a cylinder with elliptical base. The wavelength $\lambda=\frac{2 \pi v_{p}}{\omega}$ describes the spacing of the helix. A projection of this helical trajectory onto the $x$ - $y$-plane is shown in figure 2.6(b). The polarization state of the light is fully described by the orientation and shape of this polarization ellipse, whereas its extension is determined by the light intensity.

A mathematical description is achieved by eliminating any time- or position-dependence from
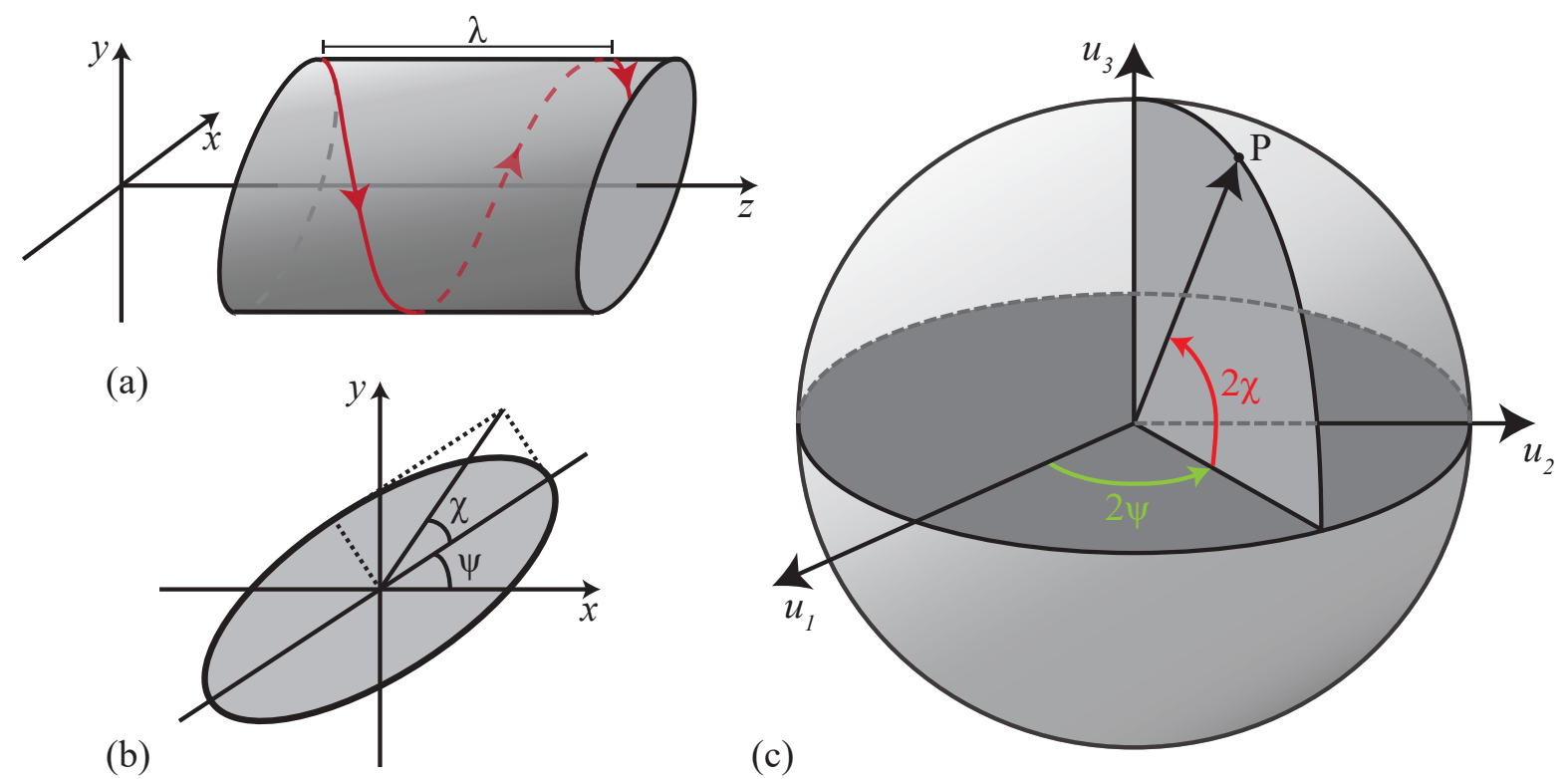

Figure 2.6: Illustration of polarization states. (a) The trajectory of the electric field vector's tip at time point $t$ is helical on an elliptical cylinder. (b) The projection of the helical trajectory onto the $x-y$ plane is an ellipse. The angles $\Psi$ and $\chi$ determine the orientation and shape of the ellipse. (c) A three-dimensional visualization of polarization states is given via the Poincaré sphere. The angles $\Psi$ and $\chi$ are related to spherical coordinates and uniquely determine the position on the sphere's surface (modified in accordance to [ST07]). 
equations (2.13) and 2.14) by combining them adequately [Hec05]

$$
\left(\frac{E_{x}}{E_{0, x}}\right)^{2}+\left(\frac{E_{y}}{E_{0, y}}\right)^{2}-2 \frac{E_{x} E_{y}}{E_{0, x} E_{0, y}} \cos \varphi=\sin ^{2} \varphi .
$$

Here, $\varphi=\varphi_{y}-\varphi_{x}$ is introduced as the phase shift between the two components. The major axis of the ellipse described by this equation encloses an angle $\Psi$ defined by [BW11]

$$
\tan (2 \Psi)=\frac{2 \frac{E_{0, y}}{E_{0, x}}}{1-\left(\frac{E_{0, y}}{E_{0, x}}\right)^{2}} \cos \varphi
$$

with the $x$-axis of the coordinate system. The ellipticity angle $\chi$, which is related to the ratio of minor and major axis, is given by BW11]

$$
\sin (2 \chi)=\frac{2 \frac{E_{0, y}}{E_{0, x}}}{1+\left(\frac{E_{0, y}}{E_{0, x}}\right)^{2}} \sin \varphi
$$

and depicted in figure 2.6(b). These two angles fully characterize the polarization state of the light. A three-dimensional visualization is given by the Poincaré sphere, shown in figure 2.6.(c). A sphere of unit radius in combination with spherical coordinates is utilized to assign each point on the sphere's surface unambiguously to a state of polarization. The polarization angle $\Psi$ can be found in the equator plane, whereas the ellipticity angle $\chi$ determines the degree of latitude. The north and south pole correspond to right-handed and left-handed circular polarization, respectively, i.e. $2 \chi= \pm 90^{\circ}$. For $\chi=0$, i.e. on the equator, all possible linear polarization states can be found, including linear polarization along the $x$-axis for $2 \Psi=0^{\circ}$ and along the $y$-axis for $2 \Psi=180^{\circ}$.

The Poincaré sphere is a powerful tool for visualizing polarization states, as also utilized in section 3.3 , but a more mathematical approach is sought for in order to describe changes of the polarization induced by optical components. As deduced in the description before and as seen from equation 2.15), the polarization state is fully characterized by the two components of the amplitude vector, i.e. $E_{0, x}, E_{0, y}, \varphi_{x}$ and $\varphi_{y}$. Forming a column vector $\boldsymbol{J}$ from this complex amplitudes as

$$
\boldsymbol{J}=\left(\begin{array}{c}
E_{0, x} e^{i \varphi_{x}} \\
E_{0, y} e^{i \varphi_{y}}
\end{array}\right)=e^{i \varphi_{x}}\left(\begin{array}{c}
E_{0, x} \\
E_{0, y} e^{i \varphi}
\end{array}\right)
$$

allows for a mathematical description of the light's polarization state Go005]. This vector is commonly referred to as the polarization vector or Jones vector. As the total intensity of the wave is typically non-relevant, the Jones vector is conventionally normalized to 1 . As can be seen from equation (2.18), the phase of the $x$-component can without loss of generality assumed 
to be 0 . This yields exemplary Jones vectors of

$$
\boldsymbol{J}=\left(\begin{array}{l}
1 \\
0
\end{array}\right)
$$

for linearly polarized light in $x$-direction and of

$$
\boldsymbol{J}=\frac{1}{\sqrt{2}}\left(\begin{array}{l}
1 \\
i
\end{array}\right)
$$

for left-handed circularly polarized light.

When an incident beam of polarization $\boldsymbol{J}_{\text {in }}$ is transmitted through an optical system which is polarization-sensitive, but maintains the plane-wave character of the wave, the polarization $\boldsymbol{J}_{\text {out }}$ of the output beam will in general be altered [ST07. Assuming that the system is linear, i.e. the components of the amplitude vector of the output wave depend linearly on those of the input wave, the relation between the polarization states is described by

$$
\boldsymbol{J}_{\text {out }}=\boldsymbol{T} \boldsymbol{J}_{\text {in }}
$$

$\boldsymbol{T}$ is a $2 \times 2$-matrix, called the Jones matrix, which fully describes the optical system and does not dependent on the polarization state of the incident beam.

An example of an optical system fulfilling the above mentioned assumptions is an optically anisotropic crystal as described in the following. The Jones formalism for calculating changes introduced by such a system is further deepened in section 3.3 .

\subsubsection{Birefringence}

This subsection describes the propagation of light through optically anisotropic materials, i.e. crystals. In general, this implies that the optical properties depend on the spatial direction, with only electrical anisotropy being considered in the following.

For a linear anisotropic dielectric medium like a crystal and an incident electro-magnetic wave, the electric displacement field $\boldsymbol{D}$ does generally not have the same direction as the electric field $\boldsymbol{E}$, but is related to it via $[\mathrm{ST07}$.

$$
D=\underline{\underline{\varepsilon}} \boldsymbol{E} .
$$

Thus, each component of $\boldsymbol{D}$ depends linearly on the components of $\boldsymbol{E}$, with the coefficients of the linear combinations forming the electric permittivity tensor $\underline{\underline{\varepsilon}}$. This tensor fully characterizes the dielectric properties of the material and can be geometrically represented by a quadratic surface, i.e. an ellipsoid [ST07]. 
For a material not exhibiting optical activity, the permittivity tensor is symmetric [ST07. With an appropriate choice of the coordinate system relative to the crystal structure, the offdiagonal elements vanish, leaving $\underline{\underline{\varepsilon}}$ to be diagonal with diagonal elements $\varepsilon_{1}, \varepsilon_{2}$ and $\varepsilon_{3}$ such that

$$
D_{i}=\varepsilon_{i} E_{i}, \quad i=1,2,3
$$

This coordinate system defines the principal axes along which $\boldsymbol{E}$ and $\boldsymbol{D}$ are parallel [BW11. Without loss of generality, the coordinate system denoted by $x, y, z$ in the following is assumed to lie along the principal axes with $\varepsilon_{1} \leq \varepsilon_{2} \leq \varepsilon_{3}$, simplifying the further considerations.

Instead of the permittivities, the principal refractive indices are the more commonly used quantities. They are related by

$$
n_{i}=\sqrt{\frac{\varepsilon_{i}}{\varepsilon_{0}}}, \quad i=1,2,3
$$

to the permittivities of non-magnetic materials, with $\varepsilon_{0}$ being the permittivity of free space. The refractive index is related to the phase velocity $v_{p}$ of the wave via

$$
n=\frac{c}{v_{p}}
$$

with $c=\frac{1}{\sqrt{\varepsilon_{0} \mu_{0}}}$ as the speed of light in vacuum and $\mu_{0}$ the vacuum permeability. Since $\boldsymbol{E}$ and $\boldsymbol{D}$ are in general not parallel, the refractive index is a function of the propagation and polarization direction of the light. Thus, also the phase velocity of a wave propagating in an anisotropic material depends on the propagation direction.

A wave propagating along one of the principal axes and being linearly polarized along one of the other two principal directions traverses the medium without a polarization change, since the electric field $\boldsymbol{E}$ has only one component and is parallel to $\boldsymbol{D}$. Hence, the polarization states along these two principal axes form a system of normal modes for this propagation direction, and an arbitrary incident polarization can be treated as the sum thereof. Due to the different phase velocities resulting from diverse refractive indices, a phase shift between the components is expected, changing the resulting polarization accordingly. Since the phase velocity increases with decreasing refractive index, the axis with the lower refractive index is usually referred to as the fast axis, in contrast to the slow axis for the higher refractive index.

To determine the phase velocities and the normal modes, i.e. the directions of polarization which are unchanged, for a given, arbitrary direction $s$ of the wave normal, a construction 
called index ellipsoid can be employed. It is found by inverting relation 2.20 as

$$
\varepsilon_{0} \boldsymbol{E}=\underline{\underline{\eta}} \boldsymbol{D}
$$

with the impermeability tensor $\underline{\underline{\boldsymbol{\eta}}}=\varepsilon_{0} \underline{\underline{\varepsilon}}^{-1}$. The symmetry of $\underline{\underline{\varepsilon}}$ implies the symmetry of $\underline{\underline{\boldsymbol{\eta}}}$, with both tensors having the same principal axes [ST07]. Employing the quadric representation Nye04 of the tensor $\underline{\underline{\boldsymbol{\eta}}}$ with respect to the coordinate system $x, y, z$ as defined by the principal axes yields an ellipsoid described by the equation

$$
\frac{x^{2}}{n_{1}^{2}}+\frac{y^{2}}{n_{2}^{2}}+\frac{z^{2}}{n_{3}^{2}}=1
$$

as illustrated in figure 2.7. The semiaxes of this ellipsoid coincide in direction with the principal axes, with their length being equal to the respective refractive indices.

This geometrical construction can be employed for determining the normal modes: The intersection of the index ellipsoid with the plane perpendicular to $s$ through the origin of the index ellipsoid is called the index ellipse. The semiaxes of this ellipse determine the refractive indices $n_{a}$ and $n_{b}$ and the corresponding phase velocities of the normal modes, with the directions defining the directions of the orthogonal vectors $\boldsymbol{D}_{\boldsymbol{a}}$ and $\boldsymbol{D}_{\boldsymbol{b}}$ BW11. The polarization directions can then be calculated by equation 2.20.

So far, no assumptions about the dimensions of the three principal refractive indices have been made. If two of the refractive indices are equal, e.g. $n_{1}=n_{2}$, the crystal is uniaxial. In this case, the index ellipsoid equals an ellipsoid of revolution and for light propagating along the $z$-axis, the refractive index does not depend on the polarization

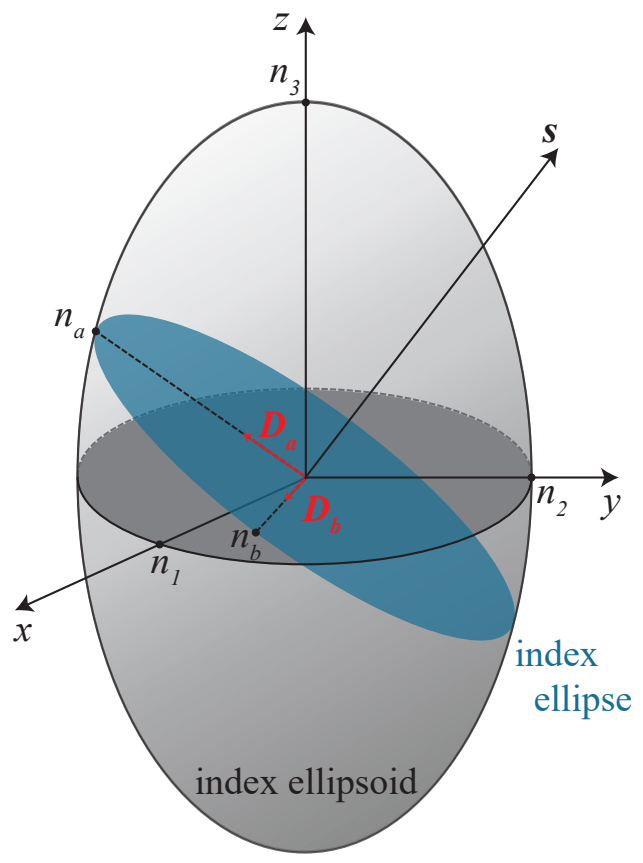

Figure 2.7: Index ellipsoid representing the impermeability tensor of an anisotropic material and index ellipse for determining normal modes for an arbitrary wave normal direction $\boldsymbol{s}$ (modified in accordance to [ST07]). of the beam. It is therefore denoted as the optical axis. $n_{1}=n_{2}=n_{o}$ is then referred to as the ordinary index, while $n_{3}=n_{e}$ is known as the extraordinary index. In case the refractive indices are all different from each other, the crystal is denoted biaxial, since it exhibits two optical axes, as explained in the following.

The identification of the two optical axes of a biaxial crystal is less obvious than in the case 
of a uniaxial crystal. Their existence, i.e. the existence of two circular sections of an ellipsoid, is shown mathematically in [BH93]. A geometric approach for determining the optical axes is presented in [ST07] and [BW11, utilizing the so-called normal surface: For each possible wave normal direction $s$, two vectors are plotted in that direction with the lengths equaling the corresponding refractive indices $n_{a}$ and $n_{b}$. The end points of these vectors for all possible $s$ describe a surface consisting of two sheets, corresponding to two normal modes [BW11].

The same surface is obtained from the dispersion relation, i.e. the dependence of the wave's angular frequency $\omega$ on the wave vector $\boldsymbol{k}$. This relation is obtained by solving Maxwell's equations

$$
\begin{aligned}
& \omega \boldsymbol{B}=\boldsymbol{k} \times \boldsymbol{E}, \\
& \omega \boldsymbol{D}=-\boldsymbol{k} \times \boldsymbol{H}
\end{aligned}
$$

where $\boldsymbol{H}$ denotes the magnetic field strength and $\boldsymbol{B}=\mu_{0} \boldsymbol{H}$ the magnetic induction. Rewriting Maxwell's equation for this setting yields [ST07.

$$
\boldsymbol{k} \times(\boldsymbol{k} \times \boldsymbol{E})+\omega^{2} \mu_{0} \underline{\underline{\varepsilon}} \boldsymbol{E}=\mathbf{0}
$$

where the wave vector and the refractive index are related via

$$
\boldsymbol{k}=\frac{\omega}{c} \boldsymbol{n}=: k_{0} \boldsymbol{n}
$$

Equation 2.21 can be rewritten in the form $\underline{\underline{\boldsymbol{M}}} \boldsymbol{E}=\mathbf{0}$ and has non-trivial solutions if the determinant of the matrix $\underline{\underline{M}}$ vanishes, i.e.

$$
n_{1}^{2} n_{2}^{2} n_{3}^{2}+n_{1}^{2} \frac{k_{1}^{2}}{k_{0}^{2}}\left(\frac{k^{2}}{k_{0}^{2}}-n_{2}^{2}-n_{3}^{2}\right)+n_{2}^{2} \frac{k_{2}^{2}}{k_{0}^{2}}\left(\frac{k^{2}}{k_{0}^{2}}-n_{1}^{2}-n_{3}^{2}\right)+n_{3}^{2} \frac{k_{3}^{2}}{k_{0}^{2}}\left(\frac{k^{2}}{k_{0}^{2}}-n_{1}^{2}-n_{2}^{2}\right)=0
$$

where $k^{2}=k_{1}^{2}+k_{2}^{2}+k_{3}^{2}$ and with $n_{i}$ as the principal refractive indices. $k_{i}$ are the components of the wave vector with respect to the principal coordinate system. The resulting dispersion relation relates $k_{0}$ and thus $\omega$ to $k_{1}, k_{2}$ and $k_{3}$, resulting in the nonlinear function $\omega\left(k_{1}, k_{2}, k_{3}\right)$. Visualizing the above equation yields the normal surface [ST07], where the refractive index is obtained from the intersection of a direction vector $s$ with the normal surface, yielding a vector of magnitude $k=k_{0} n$.

The normal surface is displayed in figure 2.8 for three different crystal types. As can be seen in figure 2.8 (a), there is no preferential direction for an isotropic crystal, and the refractive index is the same for all directions of the wave normal. Contrary, in a uniaxial crystal as depicted in figure 2.8(b), the two sheets of the normal surface intersect in two points along the $k_{3}$-axis, defining it as the optical axis. This can be directly seen from equation 2.22 when considering 
only the plane defined by $k_{2}=0$. In this case, equation 2.22 simplifies to

$$
\left(\frac{k^{2}}{k_{0}^{2}}-n_{2}^{2}\right)\left(n_{1}^{2} \frac{k_{1}^{2}}{k_{0}^{2}}+n_{3}^{2} \frac{k_{3}^{2}}{k_{0}^{2}}-n_{1}^{2} n_{3}^{2}\right)=0
$$

corresponding to a circle of radius $n_{2}$ and an ellipse with half axes $n_{1}$ and $n_{3}$. For $n_{1}=n_{2}$, these curves intersect in $k_{3} / k_{0}= \pm n_{1}$. Analogously for a biaxial crystal, the two sheets meet in four points, with only one shown in the sketch of the octant in figure 2.8 (c), defining two optical axes.

The ray direction of the wave, i.e. the direction of the group velocity, is the direction of energy transport, i.e. the direction of the Poynting vector

$$
\boldsymbol{S}=\boldsymbol{E} \times \boldsymbol{H}
$$

and is given by the gradient of the dispersion relation [BJ07]. Thus, the ray direction is perpendicular to the tangent plane to the normal surface. For any propagation direction $\boldsymbol{k}$ not pointing in the direction of one of the optical axes, there are two distinct modes, exhibiting different refractive indices, and thus giving rise to two refracted waves with different polarizations and directions. This effect is called birefringence.

For a wave traversing in the direction of one of the optical axes in a biaxial crystal, the index ellipse is by definition a circle, giving rise to infinitely many permissible directions of $\boldsymbol{D}$ for the same phase velocity, resulting in infinitely many directions of the electric field vector $\boldsymbol{E}$. This singularity can also be derived from the normal surface, since its normal at the point of the intersection of the two sheets is not defined. Hence, the ray direction is not unique, but there are infinitely many possible ray directions, which can be shown to lie on the surface of a cone [BW11]. This phenomenon is known as conical diffraction and is further discussed in subsection 2.4.3.

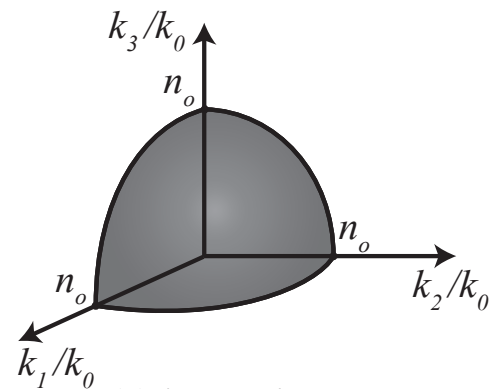

(a) isotropic

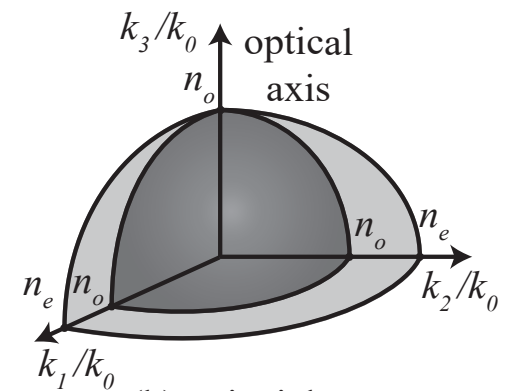

(b) uniaxial

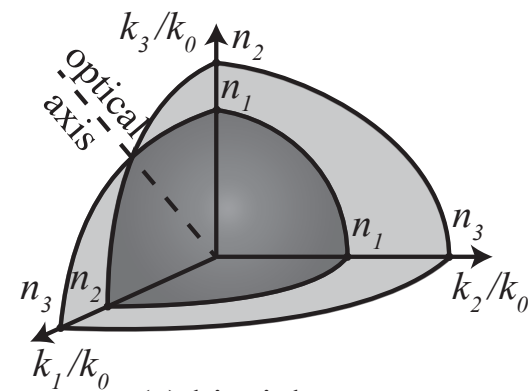

(c) biaxial

Figure 2.8: Illustration of an octant of the normal surface for (a) an isotropic crystal $\left(n_{1}=n_{2}=n_{3}=\right.$ $n_{o}$ ), (b) a uniaxial crystal $\left(n_{1}=n_{2}=n_{o}<n_{3}=n_{e}\right)$ and (c) a biaxial crystal $\left(n_{1}<n_{2}<n_{3}\right)$. The optical axis is depicted as well (modified in accordance to [ST07]). 
Birefringence can occur naturally for a material showing three different refractive indices, like e.g. potassium titanyl phosphate (KTP) as described in subsection 2.4.3, but can also be induced by the electro-optic effect. The basics thereof are outlined in subsection 2.4.4 as this effect will be employed for the control of polarization states in this thesis (cf. section 3.3.

\subsubsection{Conical diffraction}

In the early 1830s [Ham37, Hamilton first predicted the phenomenon of conical diffraction, based on the theoretical considerations outlined before. The observations by Lloyd shortly afterwards [Llo33] verified this theory, stating the occurrence of two bright rings in the focal plane when light passes a biaxial crystal along one of its optical axes. Numerous experimental works have been conducted hereafter as e.g. [BJL06], [DMB $\left.{ }^{+} 13\right]$ and $\left[\mathrm{POR}^{+}\right.$09], verifying the near-field and far-field behavior described theoretically in e.g. [Ber04]. For circularly polarized incident light, the two bright rings observed in the focal image plane get broader and exhibit intensity oscillations with increasing distance from the focal image plane. The far-field is dominated by a central spike.

The light distribution in case of an incident beam of linear polarization was described in KB08. Intensity minima and maxima can be observed in this case, breaking up the ring structure observed for circularly polarized incident light. Moreover, the resulting beam is linearly polarized everywhere, with the polarization direction depending on the position within the beam. This observation is the basis for numerous approaches of beam shaping with the help of conical diffraction. Even more elaborated systems, including more than one biaxial crystal, have been investigated theoretically [Ber10] and experimentally [Abd11.

In the following, an overview of the analytical description of the properties and characteristics of conical diffraction is introduced, based on the work of [Ber04] and [BJ07]. A biaxial crystal of length $l$ is cut perpendicular to one of its optical axes, as shown in figure 2.9. A beam with waist $w$ is assumed to enter perpendicular to the crystal's surface, i.e. along the optical axis. $n_{i}, i=1,2,3$ denote the refractive indices satisfying the relation $n_{1}<n_{2}<n_{3}$. As deduced in the previous subsection, the singularity at the intersection of the two sheets of the normal surface gives rise to the formation of a ray cone with a semiangle of [BJ07, $\mathrm{DMB}^{+} 13$ ]

$$
A=\frac{1}{2} \arctan \sqrt{\frac{\left(n_{2}^{2}-n_{1}^{2}\right)\left(n_{3}^{2}-n_{2}^{2}\right)}{n_{1}^{2} n_{3}^{2}}} .
$$

For small differences between the refractive indices, as it is usually the case in naturally biaxial crystals, this semiangle fulfills the paraxiality approximation $A \ll 1$. Then, Ber04]

$$
A \approx \frac{1}{n_{2}} \sqrt{\left(n_{2}-n_{1}\right)\left(n_{3}-n_{2}\right)}
$$


holds. The radius of the light cylinder emerging from the crystal is thus given by

$$
R_{0}=A \cdot l
$$

as displayed in figure 2.9 .

For the further theoretical description, cone-centered coordinates $\{\boldsymbol{R}, z\}$ with the origin on the crystal's entrance face as depicted in figure 2.9.(a) are introduced. To calculate the propagating wave in the crystal, the matrix operator Ber04.

$$
\boldsymbol{H}(\boldsymbol{P}, z)=\left[\frac{1}{2} P^{2} \mathbb{1}+A P\left(\begin{array}{cc}
\cos \theta_{\boldsymbol{P}} & \sin \theta_{\boldsymbol{P}} \\
\sin \theta_{\boldsymbol{P}} & -\cos \theta_{\boldsymbol{P}}
\end{array}\right)\right] \Theta(l-z)+\frac{1}{2} n_{2} P^{2} \mathbb{1} \Theta(z-l)
$$

is employed. Here, $\Theta$ denotes the Heaviside function and

$$
k^{*} \boldsymbol{P}=k^{*}\left\{P_{x}, P_{y}\right\}=k^{*} P\left\{\cos \theta_{\boldsymbol{P}}, \sin \theta_{\boldsymbol{P}}\right\}
$$

defines a transverse wave vector with $k^{*}=n_{2} k_{0}$ being the so-called crystal wave number Ber04. Due to the paraxiality assumption, $P \ll 1$ holds. The first term in equation 2.25 describes the formation of the light cone inside the crystal, i.e. for $z<l$. The propagation of light outside the crystal, i.e. for $z>l$, is represented by the second term. The resulting wave

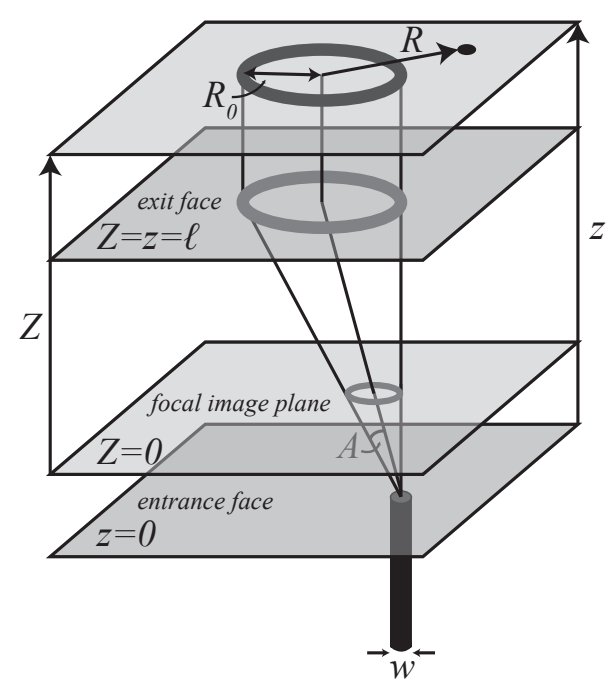

(a) three-dimensional view

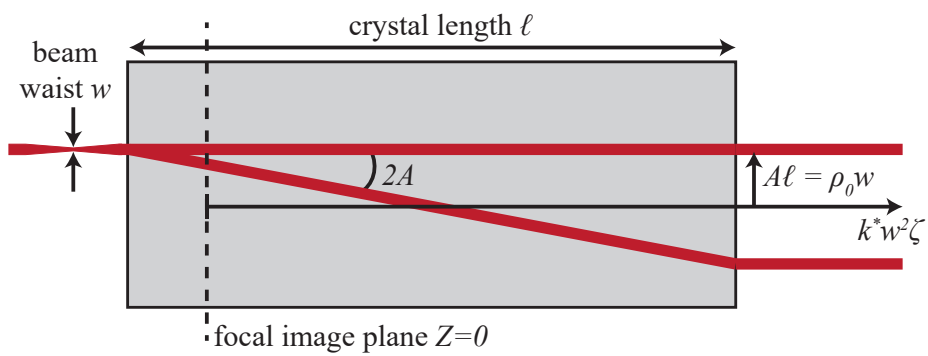

(b) two-dimensional view

Figure 2.9: Sketch of the crystal slab of length $l$ with conically diffracted beam (modified in accordance to [Ber04 and [BJL06]). (a) Three-dimensional illustration including the incident beam with waist $w$, the radius $R_{0}$ of the cone, the semi-angle $A$, the cone-centered coordinate system $\{\boldsymbol{R}, z\}$ with the origin for the $z$-axis on the entrance face of the crystal as well as the scaled axial coordinate $Z$ with the origin in the focal image plane. (b) Two-dimensional illustration of the parameter $\rho_{0}$ as well as the scaled coordinate $\zeta$ for simplifying the analysis. 
evolving from the crystal can be described by Ber04]

$$
\mathcal{D}(\boldsymbol{R}, z)=\exp \left\{-i k^{*} \int_{0}^{z} \mathrm{~d} z^{\prime} \boldsymbol{H}\left(\boldsymbol{P}, z^{\prime}\right)\right\} \mathcal{D}(\boldsymbol{R}, 0)
$$

with $\mathcal{D}(\boldsymbol{R}, 0)$ denoting the transverse profile of the incident beam. This is given by

$$
\mathcal{D}(\boldsymbol{R}, 0)=\exp \left(-\frac{R^{2}}{2 w^{2}}\right)\left(\begin{array}{l}
d_{x, 0} \\
d_{y, 0}
\end{array}\right)
$$

where $\left|d_{x, 0}\right|^{2}+\left|d_{y, 0}\right|^{2}=1$, assuming an incident Gaussian beam with beam waist $w$ and uniform polarization.

In the following, the axial coordinate $z$ is rescaled according to

$$
Z:=l+(z-l) n_{2}
$$

$Z$ denotes the distance from the focal image plane (see figure 2.9(a)), which is determined such that the optical path length for light propagating in an isotropic crystal with refractive index $n_{2}$ from that plane to the exit face is the same as for light propagating for the distance $l$ in vacuum. Note that with this definition, $z=l$ implies $Z=l$.

Furthermore, the scaled coordinates

$$
\begin{aligned}
\boldsymbol{\rho} & :=\frac{\boldsymbol{R}}{w}, \\
\rho_{0} & :=\frac{R_{0}}{w}, \\
\zeta & :=\frac{Z}{k^{*} w^{2}}
\end{aligned}
$$

are employed for simplification of the theoretical considerations, as illustrated in figure 2.9 (b). Accordingly, $R_{0}$ scales to

$$
\rho_{0}=\frac{A \cdot l}{w}
$$

which is a fundamental parameter for describing the phenomenon of conical diffraction, as it combines all dimensional variables.

Substituting equations 2.25) and 2.27) in 2.26 yields [Ber04, Ber10]

$$
\mathcal{D}(\boldsymbol{\rho}, \zeta)=\left[B_{0}\left(\rho, \rho_{0}, \zeta\right) \mathbb{1}+B_{1}\left(\rho, \rho_{0}, \zeta\right)\left(\begin{array}{cc}
\cos \theta_{\boldsymbol{\rho}} & \sin \theta_{\boldsymbol{\rho}} \\
\sin \theta_{\boldsymbol{\rho}} & -\cos \theta_{\boldsymbol{\rho}}
\end{array}\right)\right]\left(\begin{array}{c}
d_{x, 0} \\
d_{y, 0}
\end{array}\right)
$$


where

$$
\begin{aligned}
B_{0}\left(\rho, \rho_{0}, \zeta\right) & =\int_{0}^{\infty} \mathrm{d} Q Q \exp \left\{-\frac{1}{2} Q^{2}(1+i \zeta)\right\} \cos \left(\rho_{0} Q\right) J_{0}(\rho Q), \\
B_{1}\left(\rho, \rho_{0}, \zeta\right) & =\int_{0}^{\infty} \mathrm{d} Q Q \exp \left\{-\frac{1}{2} Q^{2}(1+i \zeta)\right\} \sin \left(\rho_{0} Q\right) J_{1}(\rho Q), \\
\boldsymbol{\rho} & =\rho\left(\begin{array}{c}
\cos \theta_{\rho} \\
\sin \theta_{\rho}
\end{array}\right) .
\end{aligned}
$$

Equation 2.29 is the basis for all further analysis of the beam's shaping by conical diffraction. It is immediately apparent that the conically diffracted beam consists of two parts: The first part yields an intensity modulation of the incident beam introduced by $B_{0}$ and is dominated by the behavior of the zeroth order Bessel function of the first kind $J_{0}$. Contrary, the intensity modulation of the second part is driven by the first order Bessel function of the first kind $J_{1}$ through $B_{1}$. Both parts inherit the respective properties of $J_{0}$ and $J_{1}$, like the oscillatory behavior. For example, $B_{1}$ vanishes for $\rho=0$, i.e. in the center of the cone, due to the respective characteristics of $J_{1}$.

However, the components of the conically diffracted beam are not only subject to an intensity modulation. Due to the non-isotropic nature of the crystal, components of the beam with different directions exhibit diverse polarization states. This can likewise be deduced from equation (2.29): The polarization of the first part is unchanged, as can be seen from the identity matrix. Contrary, for the second part, the polarization is affected, as indicated by the matrixvector multiplication. Since $\theta_{\rho}$ denotes the angular position within the emerging cone, the resulting polarization change is moreover dependent on the position within the beam. More precisely, the emerging polarization vector is rotated by the angle $\theta_{\boldsymbol{\rho}}$.

In case of an incident linear polarization described by

$$
\left(\begin{array}{c}
d_{x, 0} \\
d_{y, 0}
\end{array}\right)=\left(\begin{array}{c}
\cos \Psi \\
\sin \Psi
\end{array}\right),
$$

the resulting polarization of the second part in equation 2.29 is given by

$$
\left(\begin{array}{l}
d_{x} \\
d_{y}
\end{array}\right)=\left(\begin{array}{c}
\cos \left(\theta_{\rho}-\Psi\right) \\
\sin \left(\theta_{\rho}-\Psi\right)
\end{array}\right) .
$$

The resulting polarization is hence still linear, but with the direction depending on the position within the beam. Since $\Delta \theta_{\rho}=\pi$ for diametrical points of the beam, they show the same polarization state.

The overall resulting polarization, taking both beam components into consideration, depends 
on the ratio between $B_{0}$ and $B_{1}$. The position dependence allows for a selective filtering of beam components, as outlined in detail in section 3.2 .

A detailed analysis of equation (2.29) concerning the influence of the parameters $\rho_{0}$ and $\zeta$ on the final beam's intensity distribution is performed via mathematical calculations in [Ber04]. Among all, the case $\zeta=0$ has been found to be of particular interest, yielding the sharpest image of rings, contrary to the formation of intensity oscillations and an axial spike for increasing $\zeta$. Since a sharp pattern with high curvature near the intensity zero is required for an efficient depletion of fluorophores, only the case $\zeta=0$ is considered in the following.

As can be noted from equation (2.29), the intensity distribution of the conically diffracted beam depends on the chosen geometrical properties, like the crystal length $l$ and the beam waist $w$, as well as on the optical properties of the crystal, like the refractive indices, which are all included in the parameter $\rho_{0}$. Moreover, the polarization state of the incident beam as well as the selective filtering of beam components after the crystal is essential for the obtainable intensity distribution, as outlined previously. The appropriate selection of these parameters for the applicability of the conically diffracted beam as a STED beam for tomoSTED microscopy is subject to section 3.2 .

\subsubsection{Electro-optic effect}

Changes of the optical properties of a material like the refractive indices directly influence the medium-light interaction and thus the effect the medium has on polarized light. For some materials, the optical properties can be influenced by external factors, like an applied electric field or an acoustic wave traversing the medium. The former case is considered in this subsection, whereas the last subsection of this chapter deals with the acousto-optic effect.

For certain materials, an applied electric field causes a distortion of the positions, orientations or shapes of the crystal's molecules [ST07, resulting in a change of the refractive index. This change is called electro-optic effect and is usually small, but nevertheless leads to substantial phase shifts if the light propagation distance is significantly larger than the wavelength. The resulting refractive index can be written as a function of the applied electric field $E$ and can, due to the small induced variations, be expanded in a Taylor series around $E=0$. This leads to

$$
n(E)=n(0)+a_{1} E+\frac{1}{2} a_{2} E^{2}+\ldots,
$$

where $a_{1}=\left.\frac{\mathrm{d} n}{\mathrm{~d} E}\right|_{E=0}$ and $a_{2}=\left.\frac{\mathrm{d}^{2} n}{\mathrm{~d} E^{2}}\right|_{E=0}$. The higher-order terms can safely be neglected, with already the second-order term typically being orders of magnitude smaller than $n(0)$ ST07. Introducing the electro-optic coefficients $\mathfrak{r}=-2 a_{1} / n(0)^{3}$ and $\mathfrak{s}=-a_{2} / n(0)^{3}$ yields the standard 
form

$$
n(E) \approx n(0)-\frac{1}{2} \mathfrak{r} n(0)^{3} E-\frac{1}{2} \mathfrak{s} n(0)^{3} E^{2} .
$$

Generally, the coefficients $\mathfrak{r}$ and $\mathfrak{s}$ depend not only on the material, but also on the direction of the applied electric field ST07.

For most materials, equation 2.33 can be further simplified. For centrosymmetric materials like gases, liquids and certain crystals, the first derivative of $n(E)$ and thus $\mathfrak{r}$ must vanish such that it is invariant to the reversal of the electric field. These materials are known as Kerr mediums and their refractive index fulfills

$$
n(E) \approx n(0)-\frac{1}{2} \mathfrak{s} n(0)^{3} E^{2} .
$$

The index change caused by the Kerr effect or quadratic electro-optic effect, first described by John Kerr in 1875 [ST07, is hence proportional to the square of the applied electric field.

For a non-centrosymmetric material, the third term in equation 2.33 is often negligible in comparison with the second, leading to the approximation

$$
n(E) \approx n(0)-\frac{1}{2} \mathfrak{r} n(0)^{3} E
$$

with the refractive index change being proportional to the applied electric field. This is known as the Pockels effect or linear electro-optic effect, first described by Friedrich Pockels in 1893 [ST07, and is considered for the remaining of this subsection.

In the course of this work, the linear electro-optic effect is exploited for scanning as well as for polarization modification.

Electro-optic deflectors (EODs) The electro-optic effect can be employed for deflecting a beam incident on a so-called electro-optic deflector (EOD). At its simplest, an EOD consists of a crystal element with electrodes attached for generating an electric field in the crystal. As a consequence of the resulting potential change along the crystal, the refractive index is nonconstant and is, for crystals with linear electro-optic effect under a linear electric field gradient $E(x)=\kappa x$ along the width of the crystal, i.e. along the $x$-axis, described by

$$
n(x)=n(0)-\frac{1}{2} \mathfrak{r} n(0)^{3} \kappa x=: n_{0}+\eta x .
$$

Here, $n_{0}$ is the nominal index of refraction in absence of an electric field, $\eta$ is a constant depending on the applied electric field and $x$ is the distance from the side facet of the crystal. Figure 2.10 features a schematic sketch of a beam propagating along $z$ in an EOD of length $L$ with open aperture $a$, depicting the trajectory of the beam's center. In the presence of a linear 
refractive index change, the beam's displacement from the undeflected beam, i.e. the optical axis, as a function of the position $z$ along the crystal is described by [CZSS99.

$$
X(z)=\frac{1}{n_{0}} \frac{\mathrm{d} n}{\mathrm{~d} x} \frac{z^{2}}{2} .
$$

The slope of the beam's trajectory, i.e. the derivative of $X(z)$, yields the deflection angle

$$
\theta_{\text {in }}(z)=\frac{1}{n_{0}} \frac{\mathrm{d} n}{\mathrm{~d} x} z
$$

Refraction at the crystals output facet results in an external deflection angle $\theta_{\text {def }}$, obtained by evaluating $\theta_{\text {in }}(z)$ at position $z=L$ and multiplying with $n_{0}$. This results in

$$
\theta_{\mathrm{def}}=\frac{\mathrm{d} n}{\mathrm{~d} x} L
$$

The maximum beam diameter $w_{B \text {, max }}$ is determined by the deflection at the output facet of the crystal, which is given by

$$
X(L)=\frac{1}{n_{0}} \frac{\mathrm{d} n}{\mathrm{~d} x} \frac{L^{2}}{2}=\frac{1}{2} \theta_{\text {in }}(L) L .
$$

Neglecting the angle $\theta_{\text {def }}$ of the beam and thus the increased effective beam diameter,

$$
\frac{a}{2}-X(L)>\frac{w_{B}}{2}
$$

needs to be fulfilled such that the beam of diameter $w_{B}$ is not clipped at the output facet. Moreover, equation (2.36) suggests that instead of the actual parabolic trajectory, output

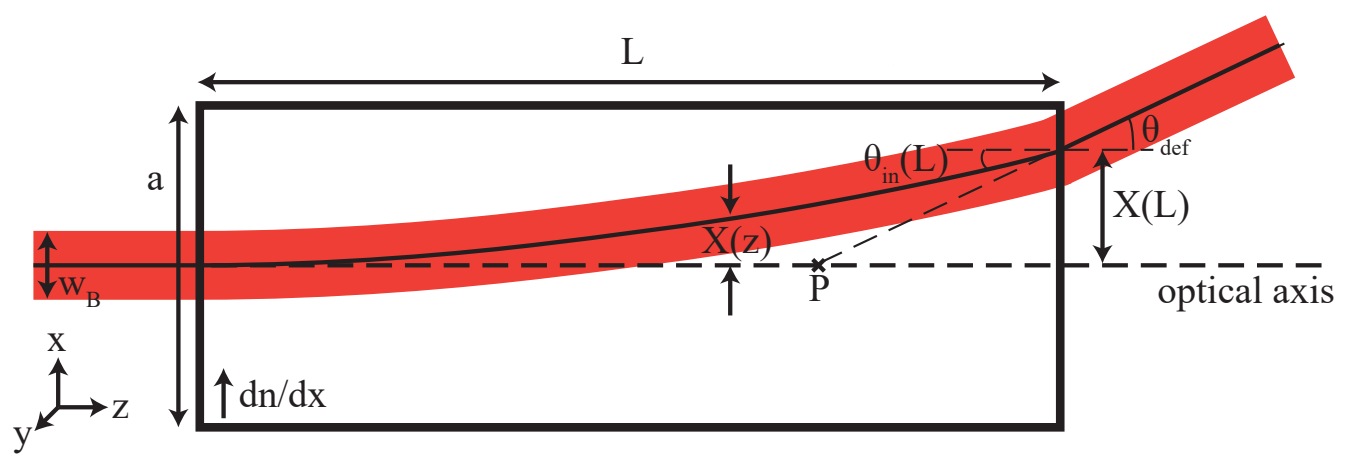

Figure 2.10: Schematic illustration of an electro-optical deflector of length $L$ and width $a$. The index of refraction is non-constant along the $x$-axis and is assumed to be $n(x)=n_{0}+\eta x$. A beam of diameter $w_{B}$ enters the crystal from the left, being deflected while traversing and undergoing refraction at the output facet. $X(z)$ denotes the position of the beam center as a function of $z, \mathrm{P}$ is the Pivot-point, $\theta_{\text {in }}(L)$ the angle of the beam's deflection at the output facet and $\theta_{\text {def }}$ denotes the resulting angle of deflection outside the crystal (modified in accordance to [Göt15]). 
angle $\theta_{\text {in }}(L)$ and displacement $X(L)$ are the same as for a beam having an abrupt deflection at distance $\frac{L}{2}$ from the output plane. For the assumption of a small deflection angle $\theta_{\text {def }}$, a similar consideration holds for the beam deflection outside the scanner. The latter can be as well described by an abrupt deflection of an angle $\theta_{\text {def }}(L)$ at a point $\mathrm{P}$ at distance

$$
L_{P}=\frac{X(L)}{\theta_{\text {def }}(L)}=\frac{1}{n_{0}} \frac{L}{2}
$$

before the output plane. This point $\mathrm{P}$ is called the pivot point.

According to equation 2.35, the refractive index gradient determines the deflection angle. Applying a field strength of sufficient order of magnitude without electrical breakdowns across side surfaces in order to achieve the desired index gradient is one of the challenging design problems in EOD fabrication. Moreover, in order to generate a spatially linearly varying refractive index, a linearly varying electric field needs to be applied to the crystal. Most commonly, this is realized by electrodes which a non-fixed geometric spacing. A linear electric field profile near the beam's transition axis of the crystal can be generated by means of quadrupole electrodes [MS04, chapter 13], as shown in figure 2.11. For a crystal with $\overline{4} 2 m$ symmetry oriented as displayed, the index gradient for an incident beam polarized along the $x$-direction and propagating along the optical axis is given by [MS04]

$$
\frac{\mathrm{d} n}{\mathrm{~d} x}=\frac{\kappa U}{a^{2}}
$$

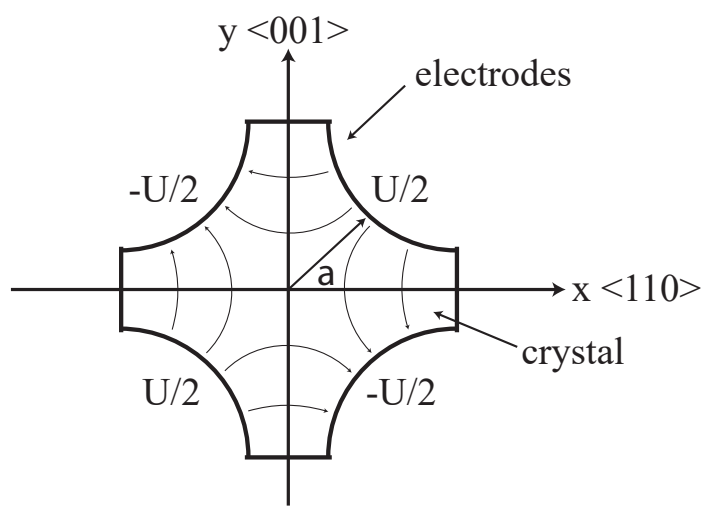

Figure 2.11: Schematic illustration of the geometry for linear electric field generation by means of quadrupole electrodes, shaped to hyperbolae. The beam propagation is perpendicular to the page with its polarization being along the $x$-axis, which is the $<110>$ crystallographic direction (modified in accordance to [MS04]). 
Here, $U$ is the applied voltage and $\kappa$ is a material-dependent constant. Hence, the beam is deflected from its initial direction by an angle

$$
\theta_{\text {def }}=\kappa \frac{L U}{a^{2}}
$$

This deflection angle depends, for a chosen crystal material, only on the length $L$ of the electrooptical material, the aperture diameter $a$ and the applied voltage $U$. Practically, $U / a$ is limited by the internal breakdown voltage, which is in the order of approximately $1000 \mathrm{~V} / \mathrm{mm}$ for fluid filled units [Con18]. Hence, for a chosen crystal material, the main parameter for increasing the maximal deflection angle is the crystal's length.

Pockels cells Apart from beam deflection, the linear electro-optic effect can also be employed for generating a voltage-controlled wave plate with adjustable phase retardation, known as Pockels cell. Depending on the direction of the electric field relative to the beam propagation direction, two main types can be distinguished: For transverse modulators, the electric field is applied in a direction perpendicular to the direction of light propagation, whereas for longitudinal Pockels cells the electric field is applied parallel thereto. Only the latter kind is considered in the following, since Pockels cells of this type are implemented in the experimental setup (see section 3.3). They consist of an anisotropic crystal, i.e. potassium dideuterium phosphate $\left(\mathrm{KD}^{*} \mathrm{P}\right)$, showing uniaxial symmetry when no electric field is applied. The optical axis is used as the longitudinal axis of the Pockels cell, with the respective refractive index given by $n_{z}=n_{e}$. The refractive index for the remaining two axes is given by the ordinary index $n_{o}$. When an electric field $E_{z}$ is applied parallel to the longitudinal, i.e. the $z$-axis, the material changes into a biaxial crystal exhibiting three different refractive indices. The $x$ - and $y$-axis are chosen according to the two normal modes considering the $z$-axis as propagation direction (cf. subsection 2.4.2), with the corresponding refractive indices given by [DH03]

$$
\begin{aligned}
& n_{x}\left(E_{z}\right)=n_{o}+\frac{1}{2} n_{o}^{3} \mathfrak{r} E_{z}=n_{o}+\Delta n^{e o}, \\
& n_{y}\left(E_{z}\right)=n_{o}-\frac{1}{2} n_{o}^{3} \mathfrak{r} E_{z}=n_{o}-\Delta n^{e o}, \\
& n_{z}\left(E_{z}\right)=n_{e}=n_{o}+\Delta n^{n} .
\end{aligned}
$$

Here, $\Delta n^{e o}=\frac{1}{2} n_{o}^{3} \mathfrak{r} E_{z}$ denotes the electro-optic birefringence, depending on the Pockels coefficient $\mathfrak{r}$ and the applied electric field. It is typically much smaller than the natural birefringence $\Delta n^{n}=n_{e}-n_{o}$, i.e. $\Delta n^{e o} \ll \Delta n^{n}$.

In the following, light traversing the Pockels cell along the $z$-axis with an incident linear polarization direction enclosing an angle of $45^{\circ}$ with respect to the $x$-axis of the Pockels cell is considered. Due to the difference of the refractive indices $n_{x}$ and $n_{y}$ in the presence of an electric field, the two components of the polarization vector are phase-shifted with respect to 
each other, with the phase shift depending on the strength of the electric field. The resulting polarization is thus in general elliptical.

The phase shift can be calculated independently for each component and is given by [ST07]

$$
\Gamma_{x, y}=n_{x, y}\left(E_{z}\right) k_{0} L
$$

with $L$ denoting the length of the Pockels cell, $k_{0}=2 \pi / \lambda_{0}$ the free space wave vector and $\lambda_{0}$ the free space wavelength of the light. The electric field is typically generated by a voltage $U_{z}$ applied over a distance $d$, i.e. $E_{z}=U_{z} / d$ with $d=L$ for the case of a longitudinal Pockels cell. This yields

$$
\begin{aligned}
\Gamma_{x, y} & =\frac{2 \pi L}{\lambda_{0}}\left(n_{o} \pm \frac{1}{2} n_{o}^{3} \mathfrak{r} \frac{U_{z}}{d}\right) \\
& =: \Gamma_{0} \pm \frac{1}{2} \pi \frac{U_{z}}{U_{\pi}}
\end{aligned}
$$

with

$$
\Gamma_{0}=\frac{2 \pi n_{o} L}{\lambda_{0}}
$$

and

$$
U_{\pi}=\frac{d}{L} \frac{\lambda_{0}}{2 \mathfrak{r} n_{o}^{3}}
$$

Consequently, the phase shift between the two orthogonal polarization components is given by

$$
\Gamma_{x}-\Gamma_{y}=\pi \frac{U_{z}}{U_{\pi}}
$$

and is thus directly proportional to the applied voltage $U_{z}$. The voltage $U_{\pi}$ is the half-wave voltage necessary to induce a phase shift of $\pi$ and is in the range of a few $\mathrm{kV}$ (for typical Pockels coefficients in the range of $10^{-10} \mathrm{~m} / \mathrm{V}$ to $10^{-12} \mathrm{~m} / \mathrm{V}$ ). The specific characteristics of the Pockels cell model employed in this work are outlined in section 3.3

\subsubsection{Acousto-optic effect}

The refractive index of a material can not only be affected by an applied electric field as considered in the latter subsection, but also by a sound wave traversing the medium. Vibrations of the molecules induced by the sound wave generate a periodic variation of the refractive index and thus a graded-index medium [ST07. The optical frequency is typically orders of magnitude higher than the acoustic frequency, enabling a quasi-stationary approach to the phenomenon. In the easiest form, parallel planes representing the index-variations induced by the acoustic wave are considered as illustrated in figure 2.12. The change of refractive index acts like an 
optical grating, diffracting an incident laser beam. The spacing between the planes is given by the wavelength $\Lambda$ of the acoustic wave. The Bragg condition for constructive interference relates the angle of incidence $\theta_{B}$ of the optical wave to the wavelengths $\Lambda$ and $\lambda$ via

$$
\sin \theta_{B}=\frac{\lambda}{2 \Lambda}
$$

with $\lambda$ being the optical wavelength in the medium. A detailed calculation (cf. [ST07]) allows for determining the ratio $\mathcal{R}$ of the intensity of reflected and incident wave yielding

$$
\mathcal{R}=\sin ^{2}\left(\sqrt{\frac{\pi^{2}}{2 \lambda_{0}^{2}}\left(\frac{L}{\sin \theta}\right)^{2} \mathcal{M} I_{s}}\right) .
$$

Here, $\lambda_{0}$ is the free space wavelength of the light, $L$ is the length of the medium and $\theta$ the angle of incidence. $\mathcal{M}$ denotes a material parameter depending inter alia on the photo-elastic constant as well as on the refractive index, whereas $I_{s}$ is the acoustic intensity. Hence, the grating's period and thus the deflection angle of the laser beam is determined by the acoustic wave's frequency as described by equation (2.40), whereas the laser beam's intensity in the first diffraction order, assuming the Bragg condition holds, can easily be modified by altering the intensity of the sound wave ( $\underline{\mathrm{MS} 04}$, chapter 12], see also equation (2.41).

An acousto-optic modulator (AOM) exploits the acousto-optic effect to deflect laser beams with switching times in the range of some hundreds of nanoseconds. It consists of an optically transparent material, like $\mathrm{TeO}_{2}$ or $\mathrm{PbMoO}_{4}$ [RB14], whose density and thus refractive index is periodically changed by a propagating soundwave as described before.

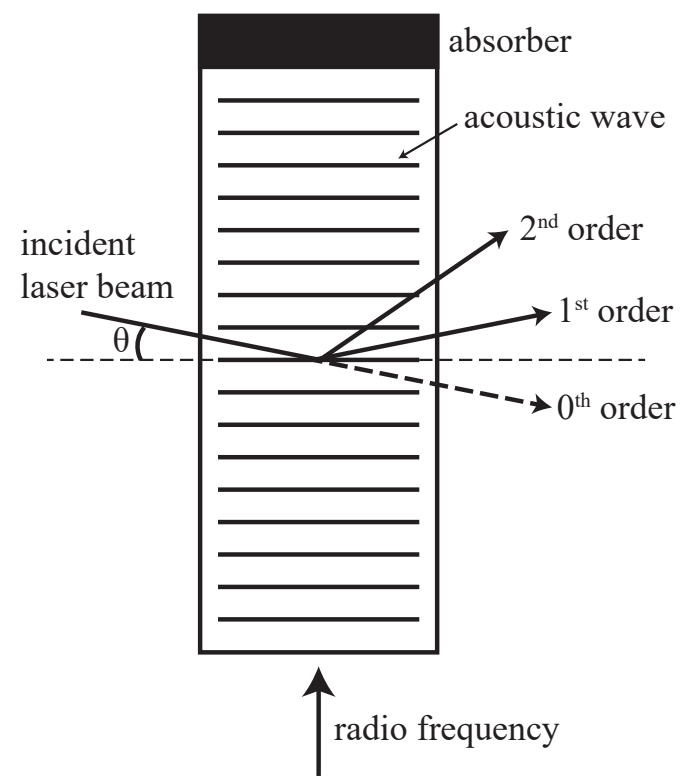

Figure 2.12: Sketch of an acousto-optic modulator (AOM). A radio-frequency induces a change in refractive index, resulting in an incident laser beam to be diffracted. The angle and intensity of the deflected beam are determined by the characteristics of the applied acoustic wave (modified in accordance to [ZLLL09]).

As seen from equations 2.40) and 2.41, for the acousto-optic effect both the angle as well as the reflection intensity depend on the wavelength of the optical wave, whereas the electro-optic effect is only indirectly wavelength dependent via dispersion. Depending on the requested application, either of the two effects can be advantageous and an appropriate choice needs to be conducted carefully. 



\section{Material and Methods}

The general experimental realization, which is chosen for investigating the potential of STED microscopy with adaptive scanning, is outlined in section 3.1. This covers inter alia the implementation of tomoSTED microscopy with ultrafast switchable depletion patterns as well as the necessary components for the novel scan system. Section 3.2 deepens the pattern generation as one of the fundamental requirements for employing tomoSTED microscopy with adaptive scanning. According to section 2.2 the polarization of the beam in the back focal plane of the objective has to match the pattern direction and is therefore a crucial parameter for the achievable resolution enhancement. Polarization states play also an important role for the pattern formation via conical diffraction, with the achievable intensity distribution depending directly on the polarization of incident and emergent beam from the biaxial crystal. Hence, the generation of polarization states is deepened in section 3.3 .

In order to derive a two-dimensionally sub-resolved image from sub-images of different pattern orientations, reconstruction algorithms are employed. An overview thereof is given in section 3.4 .

Finally, adaptive scanning is discussed in section 3.5 alongside with the necessary requirements on the experimental setup. FastRESCue as a novel variant of RESCue is presented and discussed in the view of the experimental realization. Moreover, ultrafast switchable tomoSTED depletion patterns open a variety of possible applications beyond the classical two-dimensional image acquisition. Filament tracing as one of them is introduced in section 3.5 as well.

\subsection{Experimental realization}

In the following, the experimental realization of the pulsed tomoSTED microscope with adaptive scanning is outlined. Figure 3.1 shows a schematic illustration of the setup, depicting the general beam path as well as the positioning of the optical components.

A laser diode (Exc, PicoQuant, Germany) provides excitation pulses ( $<90$ ps pulse duration) with a repetition rate of $20 \mathrm{MHz}$ at a wavelength of $640 \mathrm{~nm}$. For intensity modulation and fast switching of the laser as described in section 2.3. an acousto-optic modulator (AOM, $A A$ Opto Electronic, France) is installed. The beam is subsequently coupled into the setup via an optical fiber (Schäfter + Kirchhoff GmbH, Germany), collimated by an achromatic lens $\left(f_{13}\right)$ and de-magnified by a telescope (TC1) to obtain a beam diameter of $\approx 1.5 \mathrm{~mm}$. This is selected in accordance to the aperture of the electro-optic deflectors (EOD1, EOD2, Conoptics Inc., USA), the implementation of which will be discussed in more detail in section 3.5. Via a combination of a half-wave plate and a quarter-wave plate, the polarization of the excitation light is set circular for an efficient excitation of fluorophores. The beam is then magnified (telescope: $f_{175}$ and $\left.f_{300}\right)$ such that the back focal aperture of the objective lens (OL, UPLSAPO 100XO 
NA1.4, Olympus, Japan) is over-illuminated in order to fully exploit its numerical aperture. The focal spot generated by this 100x oil immersion objective with a numerical aperture of 1.4 is moved over the sample (S) by a galvanometer scanner (GS, Abberior Instruments GmbH, Germany).

A picosecond laser system (STED, Onefive GmbH, now part of NKT Photonics, Switzerland) with a pulse duration of approximately $600 \mathrm{ps}$, a repetition rate of $20 \mathrm{MHz}$ and a wavelength of $775 \mathrm{~nm}$ serves as STED laser. The laser power is, similarly to the excitation beam, controlled by an acousto-optic modulator (AOM, AA Opto Electronic, France). Contrary to the excitation beam, the STED beam is not coupled into the setup via a fiber, but directly used as a free beam. One of the main benefits of a fiber-coupled beam is the Gaussian intensity profile of the exiting beam. As the intensity profile of the STED laser employed already shows a Gaussian intensity distribution, a fiber coupling is renounced to for the benefit of a higher maximum laser power.

In this work, $\mathrm{AOMs}$ made of $\mathrm{TeO}_{2}$ are chosen in combination with a driver providing a radio frequency of $110 \mathrm{MHz}$. The AOMs are aligned such that the first diffraction order at the chosen radio frequency enters in the setup's beam path, whereas the zeroth diffraction order is stopped

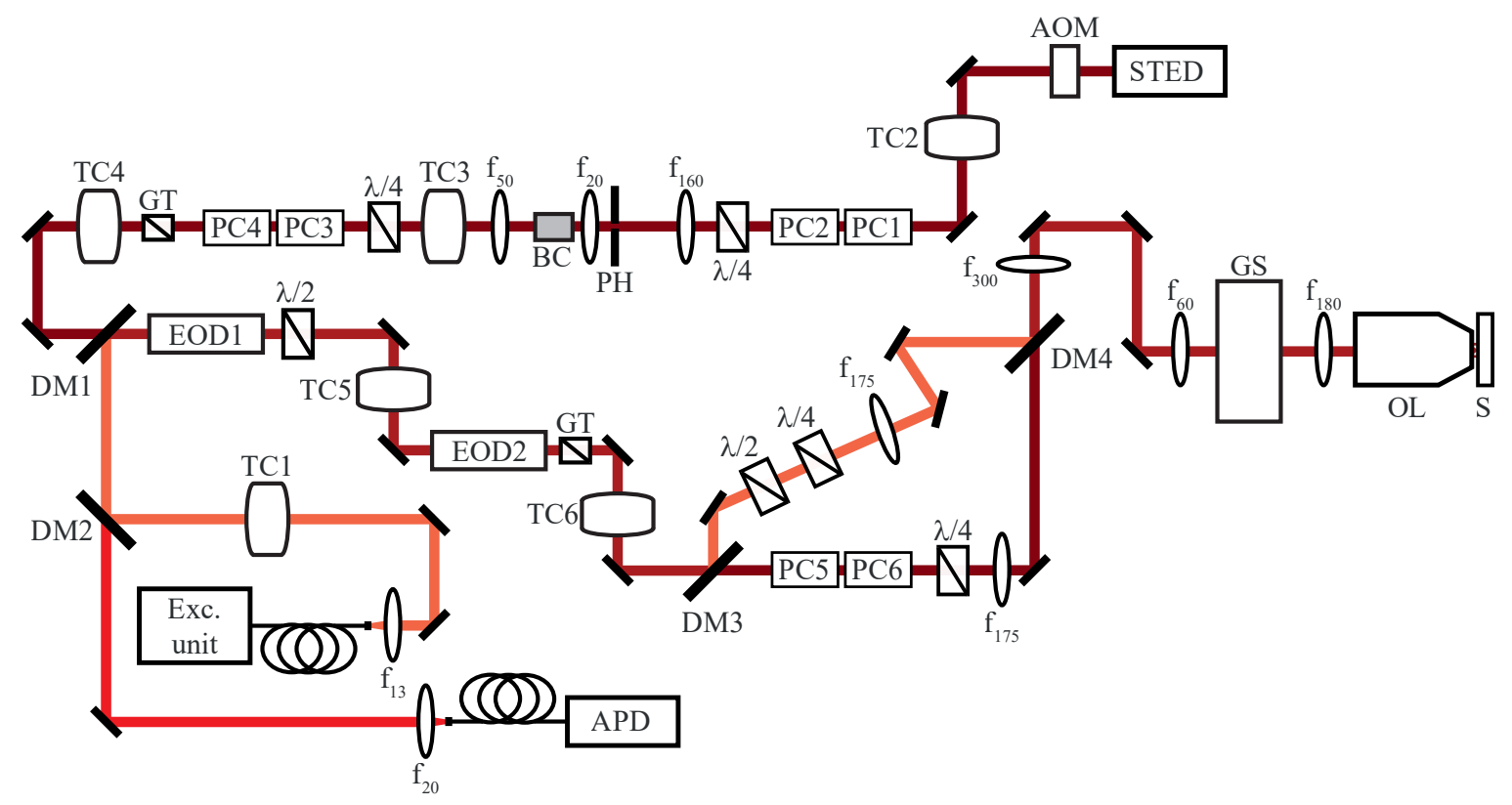

Figure 3.1: Schematic illustration of tomoSTED microscopy with fast-switchable pattern orientation. STED: depletion laser; AOM: acousto-optic modulator; Exc. unit: excitation laser, AOM and fiber coupling; TC: telescope; PC: Pockels cell; $\lambda / 2$ : half-wave plate; $\lambda / 4$ : quarter-wave plate; $f_{x}$ : lens with $x$ indicating the respective focal length in $\mathrm{mm}$; PH: pinhole of diameter $100 \mu \mathrm{m}$; BC: biaxial crystal; GT: Glan-Thompson polarizer; DM: dichroic mirror; EOD: electro-optic deflector; APD: avalanche photo diode; GS: galvanometer scanner; OL: objective lens; S: sample 
by a beam block. A deflection efficiency of up to $95 \%$ can be reached, meaning that $95 \%$ of the input laser power is diffracted into the first order.

The main component in the STED beam path is the optical element for the depletion pattern generation. In a classical STED microscope, the doughnut-shaped STED focus is generated by a polymeric phase plate with an imprinted varying thickness. This introduces a helical phase retardation to the transmitted beam, resulting in the desired focal intensity distribution. The phasemask necessary to generate a tomoSTED depletion pattern exhibits a phase step of $\pi$ (see figure 2.3 (c)). In the tomoSTED implementation described in [Krü17, a Spatial Light Modulator has been utilized to generate this one-dimensional depletion pattern. The individual images with one-dimensional resolution increase have been acquired line-wise, reducing bleaching and drifting artifacts.

Within this project, a faster implementation of the pattern rotation is sought. To achieve a pixel-wise rotation of the depletion pattern, i.e. to acquire each pixel of the recorded image repetitively for each depletion pattern orientation before the galvanometer scanner moves the focal spot to the next pixel, the pattern rotation has to be accomplished in the temporal range of the pixel dwell time, which is typically in the order of some microseconds. A Spatial Light Modulator, as employed in [Krü17, has a switching frequency in the range of $\approx 200 \mathrm{~Hz}$ and is thus too slow for this application.

In subsection 2.4.3, the basic properties of the phenomenon of conical diffraction and its potential for depletion pattern generation were outlined. This is the method of choice for this project and is described in the following. A potassium titanyl phosphate (KTP) crystal (BC, Conerefringent Optics S.L., Spain) is used as biaxial crystal and implemented in the STED beam path. The necessary requirements for the crystal choice are outlined in section 3.2. In order to achieve the beam diameter concluded therein, a telescope $\left(f_{160}\right.$ and $\left.f_{20}\right)$ is employed. A pinhole of diameter $100 \mu \mathrm{m}(\mathrm{PH})$, placed in the focal plane of the telescope's first lens, is used to eliminate diffraction rings around the center part of the beam occurring due to imperfections of the optical components. A lens $\left(f_{50}\right)$ after the biaxial crystal ensures imaging of the conical diffraction pattern and two further telescopes (TC3, TC4) are used to enlarge the beam diameter in order to meet the aperture of the EODs. The polarization of the incident beam is controlled by a pair of Pockels cells (PC1, PC2, Leysop Ltd., United Kingdom) in combination with high-voltage amplifiers (Matsusada Precision Inc., Japan), as described further in section 3.3. Analogously, another Pockels cells pair (PC3, PC4) in addition with a GlanThompson-Prism (GT, Thorlabs Inc., USA) is utilized for setting and filtering the polarization state after the biaxial crystal. The achievable switching frequency for the pattern orientation with this implementation is deduced at the end of section 3.3 .

The STED and the excitation beam are superimposed by a dichroic mirror (DM1, AHF ana- 
lysentechnik $A G$, Germany) before passing the EODs. For a most efficient depletion by the STED beam, the polarization of the STED beam must be adjusted depending on the pattern orientation as described in section 2.2 As the optimum polarization for excitation and depletion beam thus differ, the two beams are split after passing the EODs by another dichroic mirror (DM3, AHF analysentechnik AG, Germany). Similarly to the excitation beam, the STED beam is enlarged to a beam diameter of $\approx 2.4 \mathrm{~mm}$ in order to properly illuminate the objective lens. A third pair of Pockels cells (PC5, PC6) allows for a fast adaptation of the STED beam's polarization, followed by a dichroic mirror (DM4, AHF analysentechnik AG, Germany) for the final co-alignment with the excitation beam. The resulting foci of excitation and STED beam overlap such that the depletion pattern's minimum lies in the center of the excitation spot. The temporal delay between excitation and STED pulse can be controlled electronically to achieve maximum depletion efficiency.

The fluorescence signal is collected through the objective lens in an epifluorescence configuration and subsequently separated from excitation and STED beam by dichroic mirrors (DM1, DM2, AHF analysentechnik $A G$, Germany). The signal is thereafter coupled into an avalanche photo diode (APD, Excelitas, USA) via a multimode fiber (Thorlabs Inc., USA) which also serves as a pinhole. Therefore, the focusing lens $\left(f_{20}\right)$ is chosen such that the fiber diameter corresponds to 1.2 Airy units.

The setup can be operated in two different configurations, which are independent of each other and are chosen depending on the measurement framework.

For image acquisition with or without pixel-wise switching, the delay as well as the synchronization of scanning and detection is configured with the Imspector software (Abberior Instruments and MPI for biophysical chemistry, Germany) in combination with a multifunction reconfigurable I/O device (PCIe-7852, National Instruments corp., USA). The Imspector software additionally provides settings for a gated detection as well as a control of the AOMs for the laser power regulation. The Pockels cells are operated and calibrated by a self-written LabVIEW (National Instruments corp., USA) routine controlling an analog output device (PCI-6733, National Instruments corp., USA). The LabVIEW program is triggered by a pulse provided by Imspector after each pixel, post-processed by a pulse generator (Quantum Composers Inc., USA) into a TTL pulse. The LabVIEW program can be set to modify the polarization states on each trigger signal as described in more detail in section 3.3 or to set a fixed, chosen polarization state.

For adaptive scanning as described in section 3.5, a more elaborated control of the scanning is needed, which cannot be performed with the Imspector software. Therefore, a self-written LabVIEW routine is employed for programming the Field-Programmable Gate Array (FPGA) on the multifunction reconfigurable $\mathrm{I} / \mathrm{O}$ device in order to allow for a fast control of the scanning and its synchronization with the detection. This program operates the Pockels cells and sends 
out a trigger signal which drives the AOMs via an electronic switch (Analog Devices, USA). In this configuration, the delay between excitation and STED pulse is set via an external home-built fast delay line. The details of the LabVIEW routine are described in section 3.5.

\subsection{Pattern generation}

According to equation $(2.29)$, the intensity distribution of the conically diffracted beam depends on the characteristics of the incident beam, namely its intensity distribution and polarization, as well as on $\zeta$ and $\rho_{0}$. The latter is directly correlated to the radius of the emerging cone and is thus influenced by the optical properties of the crystal's material, the crystal's dimensions and the beam waist of the incident beam $(2.28)$. $\zeta$ denotes the distance of the imaged plane to the focal image plane in the crystal (cf. figure 2.9(b)). As deduced in Ber04, the sharpest rings of conical diffraction can be observed for $\zeta=0$, which is thus the optimal parameter choice for all further considerations. In the experimental realization, the focal plane is projected onto the sample plane by means of a lens behind the crystal, which is placed such that its back focal plane coincides with the plane corresponding to $\zeta=0$.

The choice of a suitable parameter $\rho_{0}$ for depletion pattern generation has been subject to Vin15] with the main results summarized in the following. To analyze the formation of rings for different values of $\rho_{0}$, MATLAB (MathWorks, USA) simulations of the intensity distribution in the sample plane, based on equation (2.29), are conducted. Here and in the following, an incident beam with Gaussian intensity profile and fixed beam waist is assumed. The results are shown in figure 3.2 (a) for $\rho_{0}=20$ and 3.2 (b) for $\rho_{0}=1.3$. In both cases, the incident light is circularly polarized, i.e.

$$
\left(\begin{array}{c}
d_{x, 0} \\
d_{y, 0}
\end{array}\right)=\frac{1}{\sqrt{2}}\left(\begin{array}{l}
1 \\
i
\end{array}\right) .
$$

For $\rho_{0}=20$, the two Poggendorff bright rings are clearly distinguishable, whereas for $\rho_{0}=1.3$ only one broad ring with a considerably smaller radius can be identified. In both cases, the intensity distribution is circularly symmetric.

However, the intensity distribution does not only depend on $\zeta$ and $\rho_{0}$, but can also be modulated by the polarization state of the incoming light as can be seen from equation 2.29). Choosing linear polarization instead of circular polarization, i.e.

$$
\left(\begin{array}{l}
d_{x, 0} \\
d_{y, 0}
\end{array}\right)=\left(\begin{array}{l}
1 \\
0
\end{array}\right),
$$

yields an intensity distribution without rotational symmetry (see figure 3.2(c)).

This observation can be explained theoretically by rewriting equation (2.29) for the case of 
incident light with linear polarization of direction $\Psi$

$$
\begin{aligned}
\mathcal{D}(\boldsymbol{\rho}, \zeta) & \approx B_{0}\left(\rho, \rho_{0}, \zeta\right)\left[\mathbb{1}+\left(\begin{array}{cc}
\cos \theta_{\boldsymbol{\rho}} & \sin \theta_{\boldsymbol{\rho}} \\
\sin \theta_{\boldsymbol{\rho}} & -\cos \theta_{\boldsymbol{\rho}}
\end{array}\right)\right]\left(\begin{array}{c}
\cos \Psi \\
\sin \Psi
\end{array}\right) \\
& =2 B_{0}\left(\rho, \rho_{0}, \zeta\right) \cos \left(\Psi-\frac{\theta_{\rho}}{2}\right)\left(\begin{array}{c}
\cos \left(\frac{\theta_{\rho}}{2}\right) \\
\sin \left(\frac{\theta_{\rho}}{2}\right)
\end{array}\right) .
\end{aligned}
$$

Here, $\theta_{\boldsymbol{\rho}}$ denotes the angular position within the emerging cone (cf. (2.32)). The approximation $B_{0}\left(\rho, \rho_{0}, \zeta\right) \approx B_{1}\left(\rho, \rho_{0}, \zeta\right)$ is employed, which holds for thick crystal slabs compared to the

(a) 40

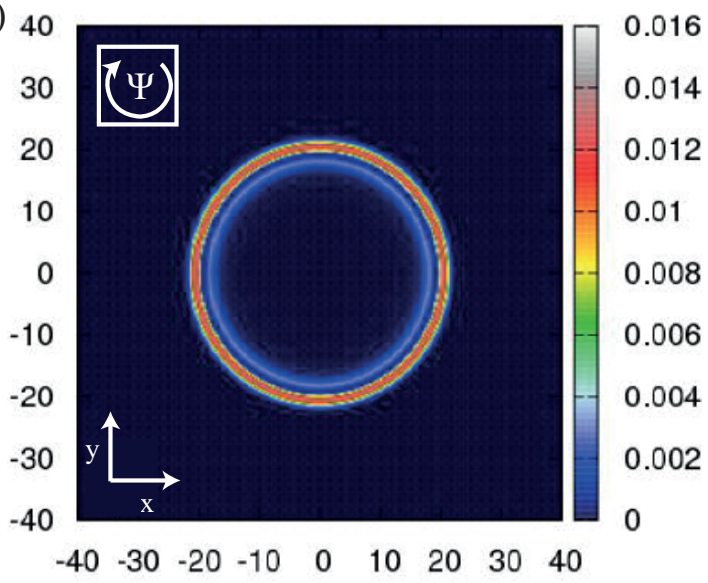

(c)

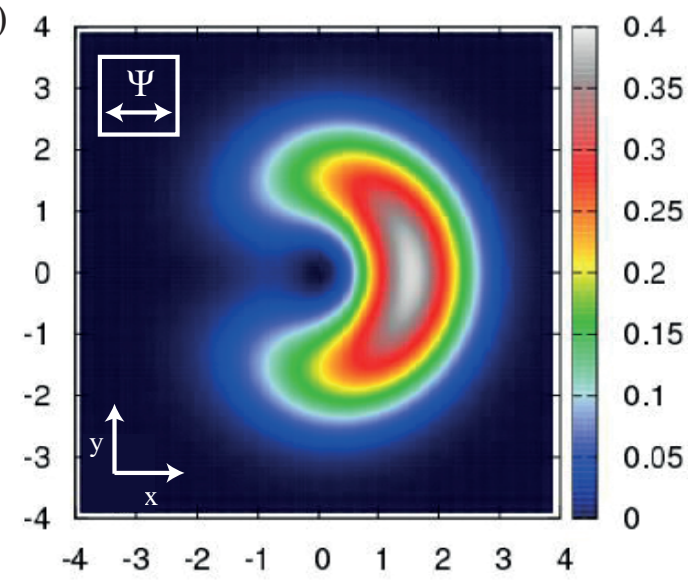

(b) 4

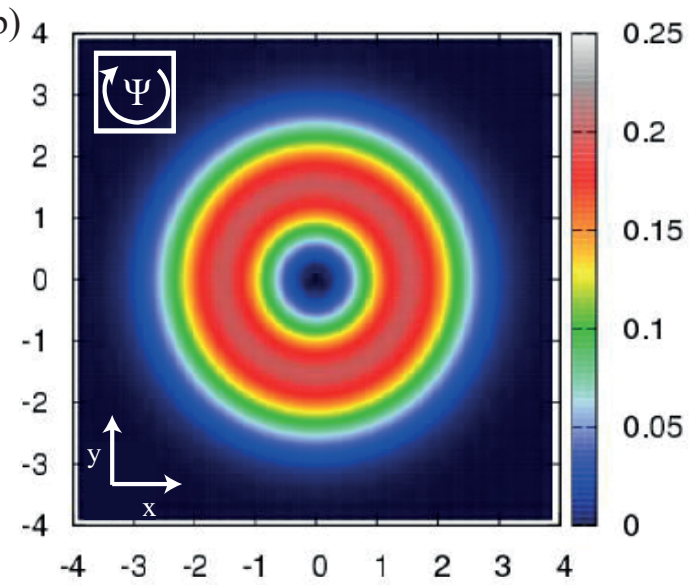

(d) 4

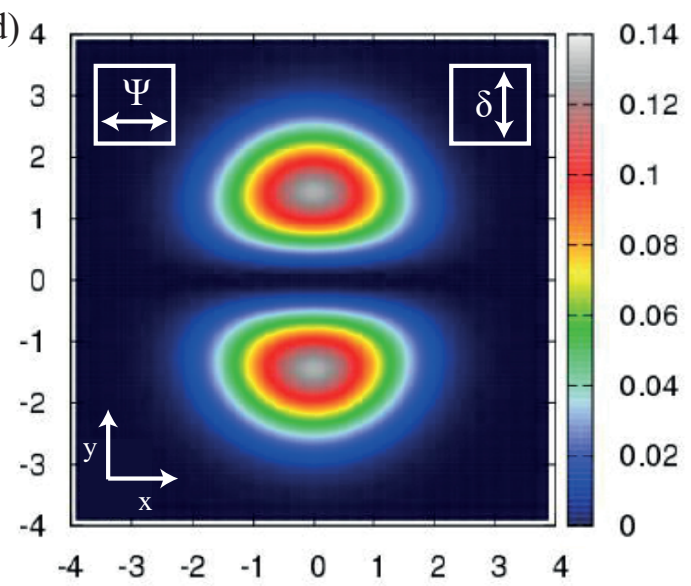

Figure 3.2: Simulation of intensity distributions at $\zeta=0$ for varying $\rho_{0}$ and different polarization. The maximal intensity of the incident Gaussian beam is normalized to 1 , the scale of $x$ - and $y$-axis is arbitrary, but consistent for all simulations. (a,b) The polarization of the incoming light is circular. The radius of the emerging cone is significantly higher in (a) $\rho_{0}=20$ than in (b) $\rho_{0}=1.3$ (note the different scales for (a) and (b)). (c,d) For a linear polarization of the incident beam (in this case, $\Psi=0$ ), the resulting intensity distribution is not rotationally symmetric (here, $\rho_{0}=1.3$ ). (d) An additional polarizer enclosing an angle $\delta=\pi / 2$ with the $x$-axis is added behind the crystal, yielding the tomoSTED depletion pattern. 
beam waist $w$ of the incident beam Ber04]. Noteworthy, the factor $\cos \left(\Psi-\frac{\theta_{\rho}}{2}\right)$ introduces changes in the intensity depending on the position within the beam, meaning that the intensity distribution is no longer circularly symmetric. Zero intensity is expected for an angle of $\theta_{\rho}=2 \Psi+\pi$, and an intensity maximum for $\theta_{\rho}=2 \Psi$. For the initial polarization enclosing for example an angle $\Psi=0$ with the $x$-axis, an intensity maximum can be observed for $\theta_{\boldsymbol{\rho}}=0$, whereas a minimum is attained for $\theta_{\rho}=\pi$. This is also visible in the simulation results in figure $3.2(\mathrm{c})$.

Anyhow, the intensity distribution depicted in figure 3.2(c) does not yet resemble the desired 1D depletion pattern with an intensity minimum along one axis, e.g. minimal intensity for $\theta_{\boldsymbol{\rho}}=0$ and $\theta_{\boldsymbol{\rho}}=\pi$. A closer look on equation (3.1) reveals that the polarization of the emerging cone is non-homogeneous: Not only the intensity depends on the angular position $\theta_{\boldsymbol{\rho}}$, but also the polarization direction. Diametrical points exhibit an angle difference of $\Delta \theta_{\rho}=\pi$, resulting in an angle difference of $\pi / 2$ for the polarization directions. Thus, to generate a $1 \mathrm{D}$ intensity distribution, a polarization analyzer after the crystal can be employed. Its theoretical description is represented by the Jones matrix

$$
T_{\mathrm{PSA}}(\delta)=\left(\begin{array}{ll}
\cos ^{2} \delta & \cos \delta \sin \delta \\
\sin \delta \cos \delta & \sin ^{2} \delta
\end{array}\right),
$$

with $\delta$ denoting the orientation of the transmitted polarization direction. Including the Jones matrix in equation 3.1 yields

$$
\mathcal{D}_{\text {res }}(\boldsymbol{\rho}, \zeta)=2 B_{0}\left(\rho, \rho_{0}, \zeta\right) \cos \left(\Psi-\frac{\theta_{\boldsymbol{\rho}}}{2}\right) \cos \left(\delta-\frac{\theta_{\boldsymbol{\rho}}}{2}\right)\left(\begin{array}{c}
\cos \delta \\
\sin \delta
\end{array}\right)
$$

which simplifies to

$$
\mathcal{D}_{\text {res }}(\boldsymbol{\rho}, \zeta)=B_{0}\left(\rho, \rho_{0}, \zeta\right) \sin \left(2 \Psi-\theta_{\boldsymbol{\rho}}\right)\left(\begin{array}{l}
\sin \Psi \\
-\cos \Psi
\end{array}\right)
$$

for $\delta=\Psi-\frac{\pi}{2}$. Consequently, for an incident linearly polarized beam with polarization orientation along $\Psi$ and a polarization analyzer with transmission direction along $\Psi-\frac{\pi}{2}$, minima in the intensity distribution are expected for $\theta_{\rho}=2 \Psi+k \pi, k \in \mathbb{Z}$. Thus, a line of zero intensity, as demanded for a $1 \mathrm{D}$ depletion pattern, forms for $\theta_{\rho}=2 \Psi$. It has to be noted that the polarization of the emerging beam depends on the initial polarization and has to match the direction of the line of zero intensity for an efficient depletion pattern formation, as described in section 2.2. Consequently, the polarization of the emerging beam needs to be rotated subsequently.

The resulting intensity distribution is exemplarily illustrated in figure 3.2 (d) for $\Psi=0$ and $\delta=\frac{\pi}{2}$. It resembles the requested 1D depletion pattern and exhibits a horizontal line of zero 
intensity. Likewise, a vertically oriented pattern can be realized for $\Psi=\frac{3 \pi}{4}$ and $\delta=\frac{\pi}{4}$.

For an optimal choice of $\rho_{0}$ and a quantitative analysis, equation (3.2) is implemented in Matlab. The intensity distribution in the focal plane is calculated from the electric field in the pupil plane of the objective lens according to vectorial diffraction theory [RW59], employing a Matlab routine by A. Schönle and J. Keller-Findeisen. The total intensity of the incident beam is normalized. Since the resolution enhancement depends on the curvature near the depletion pattern's minimum (cf. equation (2.11), the curvature of the pattern is determined and graphically displayed in figure 3.3 (a) for different $\rho_{0}$. As can be seen from the inset, the highest curvature and thus highest resolution enhancement is expected for $\rho_{0} \approx 1$.3. For larger $\rho_{0}$, more than one ring with increasing radius forms, resulting in a decreasing pattern steepness of the depletion focus as also seen from the comparison of figure 3.2 (a) and 3.2 (b). For smaller $\rho_{0}$, the ring gets broader, leading to a loss of intensity at the back focal aperture.

For $\rho_{0}=1.3$, the resulting intensity profile $(x=0, y)$ (cf. figure $3.2(\mathrm{~d})$ ) of the depletion pattern in the focal plane is compared to the pattern conventionally generated by a $0 / \pi$ phase plate. The results are displayed in figure 3.3(b). Both profiles show a high similarity in the central region. Noteworthy are the more pronounced side-lobes of the conventionally generated depletion pattern, yielding a slightly lower peak intensity of the maxima for the same total power. Nevertheless, a comparable resolution enhancement is expected for employing the conventionally generated pattern and the depletion pattern generated by conical diffraction, which was also verified experimentally in [Vin15].

(a)

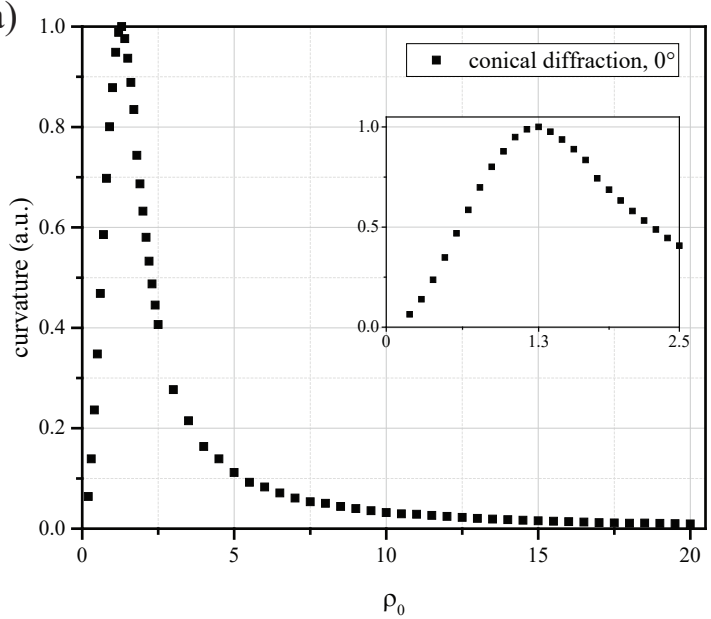

(b)

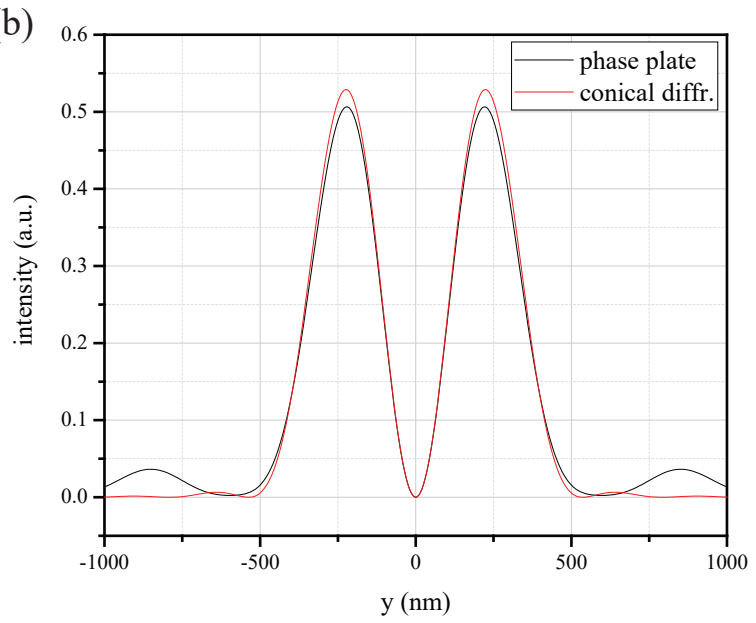

Figure 3.3: (a) Simulation of achievable curvature with intensity distribution as in figure 3.2 (d) as a function of $\rho_{0}$. The power of the incident beam is normalized to 1 . The inset is a magnification for better readability in the vicinity of the maximal curvature. (b) Comparison of intensity profiles for a 1D depletion pattern conventionally generated by a $0 / \pi$ phase plate and a pattern generated by using a conically diffracted beam with $\rho_{0}=1.3$. The total power is normalized. The profiles $(x=0, y)$ show a high similarity in the vicinity of the central minimum. 
A requested value of $\rho_{0}$ can be experimentally attained by a suitable combination of the crystal's properties and the beam waist of the incident beam (cf. equation (2.28)). The crystal element utilized within this project, shown in figure 3.4 , is made of potassium titanyl phosphate (KTP) and cut perpendicular to one of its optical axes. It has an entrance face of $3 \times 4 \mathrm{~mm}^{2}$ and a length of $11 \mathrm{~mm}$. Its refractive indices can be determined via the Sellmeier equations [Uni13] to $n_{1} \approx 1.75, n_{2} \approx 1.76$ and $n_{3} \approx 1.85$ for $\lambda_{0}=775 \mathrm{~nm}$. Thus, the refractive indices obey the assumptions made in subsection 2.4.3. The radius of the emergent cone is hence $R_{0} \approx 172 \mu \mathrm{m}$ following equation 2.23 and (2.24). The corresponding beam waist $w$ is determined by the given values of $\rho_{0}$ and $R_{0}$ via equation 2.28 and can be experimentally realized by inserting a suitable telescope in the beam path. The intensity distribution of the beam is measured via a CCD camera (Point Grey Research, Inc., USA) and the

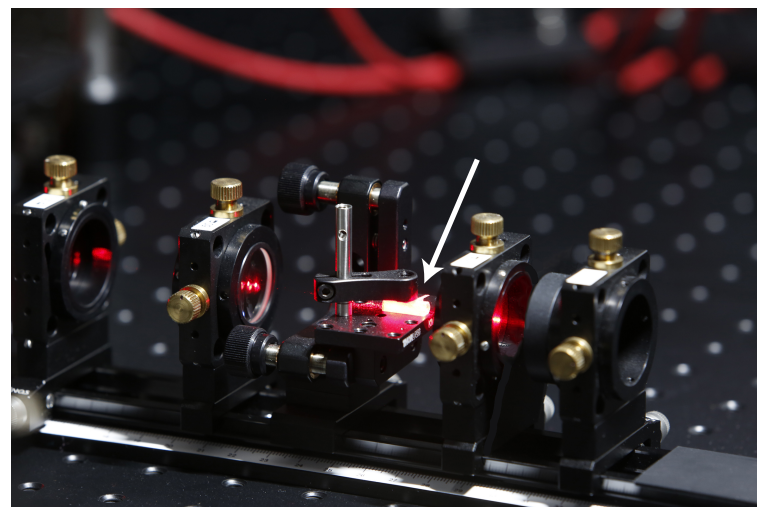

Figure 3.4: Image of the KTP crystal in the setup. resulting value of $w$ is measured to $w=(128 \pm 13) \mu \mathrm{m}$. This yields $\rho_{0}=1.35 \pm 0.14$ which is in good agreement with the demanded value $\rho_{0}=1.3$.

As described beforehand, the orientation of the depletion pattern is solely determined by $\Psi$ and $\delta$, i.e. by the polarization direction of the incident light and the transmission direction of the polarization analyzer. For realizing a specific pattern rotation, these directions have to be adapted accordingly. Experimentally, there are different options for controlling the polarization direction of a laser beam, e.g. by utilizing wave plates or Glan-Thompson-prisms as polarization analyzers as demonstrated in Vin15. Since the scope of this work is to obtain the fastest possible pattern and thus polarization rotation, only the following implementation is considered, which is schematically displayed in figure 3.5 .

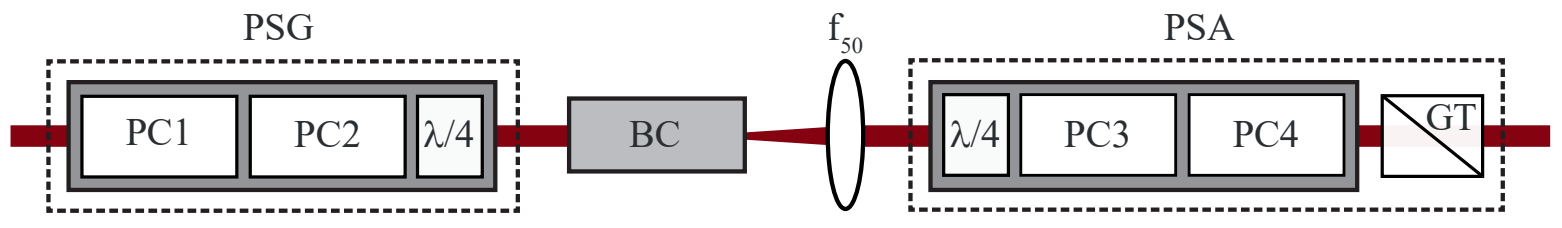

Figure 3.5: Schematic layout for depletion pattern generation. The incident beam's polarization is modified by the Polarization State Generator (PSG), consisting of a pair of Pockels cells (PC1, PC2) and a quarter-wave plate. The beam subsequently undergoes conical diffraction in the biaxial crystal (BC) with the focal plane selection being performed by the lens $f_{50}$. A Polarization State Analyzer (PSA) is utilized for selecting appropriate beam components for the depletion pattern generation. It is in this implementation composed of a quarter-wave plate, a pair of Pockels cells (PC3, PC4) as well as a static Glan-Thompson prism as polarizer (modified in accordance to [Krü17] and Bio14]). 
The polarization direction of the incoming beam is modified via a Polarization State Generator (PSG), consisting of a pair of Pockels cells (PC1, PC2) and a quarter-wave plate. This configuration allows for a setting of all possible linear polarization states with switching rates up to $300 \mathrm{kHz}$, as further deepened in section 3.3 . The linearly polarized beam subsequently undergoes conical diffraction in the biaxial crystal (BC). The focal plane $\zeta=0$ with the sharpest rings is imaged by a lens $\left(f_{50}\right)$ after the crystal. The Polarization State Analyzer (PSA) is utilized for the selection of the desired beam components as outlined previously. It is composed of a quarter-wave plate and a Pockels cells pair (PC3, PC4) for rotating the polarization accordingly before the beam passes a static linear polarizer for filtering the undesired beam components.

Hence, the generation and switching of the depletion pattern depends only on the fast control of the Pockels cells, which determine all relevant polarization states. Since the polarization of the emerging beam has to match the direction of the depletion pattern's minimum and consequently needs to be rotated accordingly, another Pockels cells pair (PC5, PC6, cf. figure 3.1) in combination with a quarter-wave plate, analog to the Polarization State Generator, is employed.

\subsection{Generation of polarization states}

As outlined in the previous section, the generation and modulation of polarization states plays an essential role for the depletion pattern generation via conical diffraction. The intensity distribution of the beam is determined by the polarization state of the incident beam before undergoing diffraction and by the appropriate selection of beam components afterwards. Furthermore, the emergent beam's polarization has to be adapted according to the pattern direction. This poses the necessity of a fast polarization state control in a

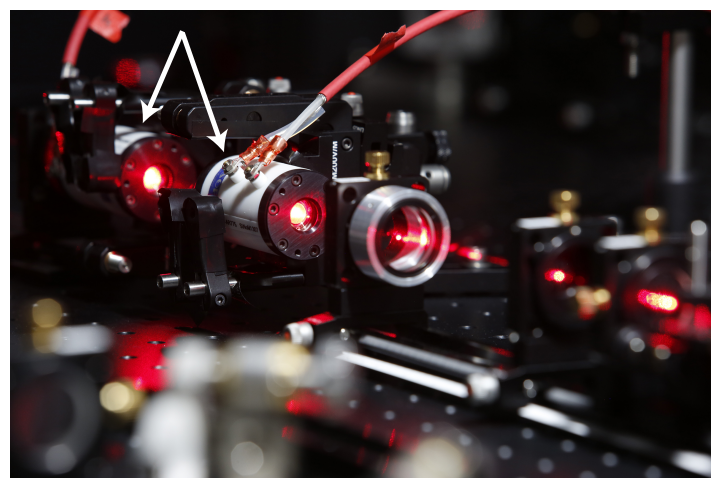

Figure 3.6: Image of a Pockels cells pair. fraction of the pixel dwell time, i.e. in the range of some $\mu$ s, since solely the speed of polarization rotation determines the speed of the pattern rotation. Thus, Pockels cells are employed for the generation of polarization states.

The theoretical basics of the underlying Pockels effect were outlined in subsection 2.4.4. In the following, the details of the experimental implementation like the orientation of the Pockels cells' fast axes will be deduced and discussed.

For all following considerations, it is assumed that the refractive indices of the Pockels cells' material under an applied electric field are given by $n_{x}$ and $n_{y}$. Here, $n_{y}$ corresponds to the fast 
axis, i.e. $n_{x}>n_{y}$ (see subsections 2.4.2 and 2.4.4 for details). Contrary to the considerations in subsection 2.4.4 the fast axis does not always coincide with the $y$-axis of the laboratory coordinate system. In a more general setting, this axis encloses an angle $\theta$ with the $x$-axis of the laboratory coordinate system, resembling a rotation of the Pockels cell around the $z$-axis. The angle $\theta$ is the free parameter to be determined for an experimental implementation. The effect of the Pockels cell on the polarization state of the incident beam is further determined by the phase shift $\Gamma$. The latter is imprinted onto beam components with orthogonal polarization directions with respect to the principal axes corresponding to $n_{x}$ and $n_{y}$. This phase shift depends on the voltage applied to the respective Pockels cell (cf. equation 2.39) and is therefore controlled electronically.

For a mathematical description, the Jones formalism is employed. Linear retarders, as e.g. Pockels cells, are uniquely characterized by two parameters as introduced before: the angle $\theta$ enclosed between the fast axis and the $x$-axis as well as the phase difference $\Gamma$ between orthogonal polarization directions. The Jones matrix for such an inclined index ellipsoid (cf. figure 2.7] is given by [Här17]

$$
\boldsymbol{T}_{\Gamma}(\theta)=\left(\begin{array}{cc}
e^{i \frac{\Gamma}{2}} \cos ^{2} \theta+e^{-i \frac{\Gamma}{2}} \sin ^{2} \theta & \left(e^{i \frac{\Gamma}{2}}-e^{-i \frac{\Gamma}{2}}\right) \cos \theta \sin \theta \\
\left(e^{i \frac{\Gamma}{2}}-e^{-i \frac{\Gamma}{2}}\right) \cos \theta \sin \theta & e^{i \frac{\Gamma}{2}} \sin ^{2} \theta+e^{-i \frac{\Gamma}{2}} \cos ^{2} \theta
\end{array}\right) .
$$

The polarization state of the emerging beam can hence be calculated by a matrix-vector multiplication (see equation 2.19).

\section{Polarization State Generator}

The Polarization State Generator for the generation of a linear polarization state with the requested orientation consists of a combination of two Pockels cells and a quarter-wave plate. The effect of this configuration on an incident linearly polarized beam is graphically outlined in figure 3.7 and explained in detail in the following. It is noted here that the configuration chosen in this work differs from the previously presented arrangements in Vin15] and Krü17] by the additional quarter-wave plate. As discussed later, this yields a higher stability when aligning for linear polarization states and is therefore preferable for the intended application.

For a beam with initial polarization

$$
\boldsymbol{J}_{\text {in }}=\left(\begin{array}{l}
1 \\
0
\end{array}\right)
$$

the angular positions $\theta_{1}$ and $\theta_{2}$ of the Pockels cells are chosen to be $45^{\circ}$ and $0^{\circ}$ (see figure 3.7(a)). 
The polarization state of the emergent beam can then be described by

$$
\boldsymbol{J}_{\Gamma_{1}, \Gamma_{2}}=\boldsymbol{T}_{\lambda / 4}\left(45^{\circ}\right) \boldsymbol{T}_{\Gamma_{2}}\left(0^{\circ}\right) \boldsymbol{T}_{\Gamma_{1}}\left(45^{\circ}\right) \boldsymbol{J}_{\text {in }}
$$

where $\Gamma_{1}, \Gamma_{2}$ denote the respective phase shift for the two Pockels cells. $\boldsymbol{T}_{\lambda / 4}\left(45^{\circ}\right)$ stands for the Jones matrix corresponding to the quarter-wave plate with its fast axis enclosing an angle of $45^{\circ}$ with the $x$-axis (see figure $3.7(\mathrm{a})$ ).

After passing the first Pockels cell of this configuration, the Jones vector of the output wave is described by

$$
\boldsymbol{J}_{\Gamma_{1}}=\boldsymbol{T}_{\Gamma_{1}}\left(45^{\circ}\right)\left(\begin{array}{l}
1 \\
0
\end{array}\right)=\left(\begin{array}{cc}
\cos \left(\frac{\Gamma_{1}}{2}\right) & i \sin \left(\frac{\Gamma_{1}}{2}\right) \\
i \sin \left(\frac{\Gamma_{1}}{2}\right) & \cos \left(\frac{\Gamma_{1}}{2}\right)
\end{array}\right)\left(\begin{array}{l}
1 \\
0
\end{array}\right)=\left(\begin{array}{c}
\cos \left(\frac{\Gamma_{1}}{2}\right) \\
i \sin \left(\frac{\Gamma_{1}}{2}\right)
\end{array}\right) .
$$

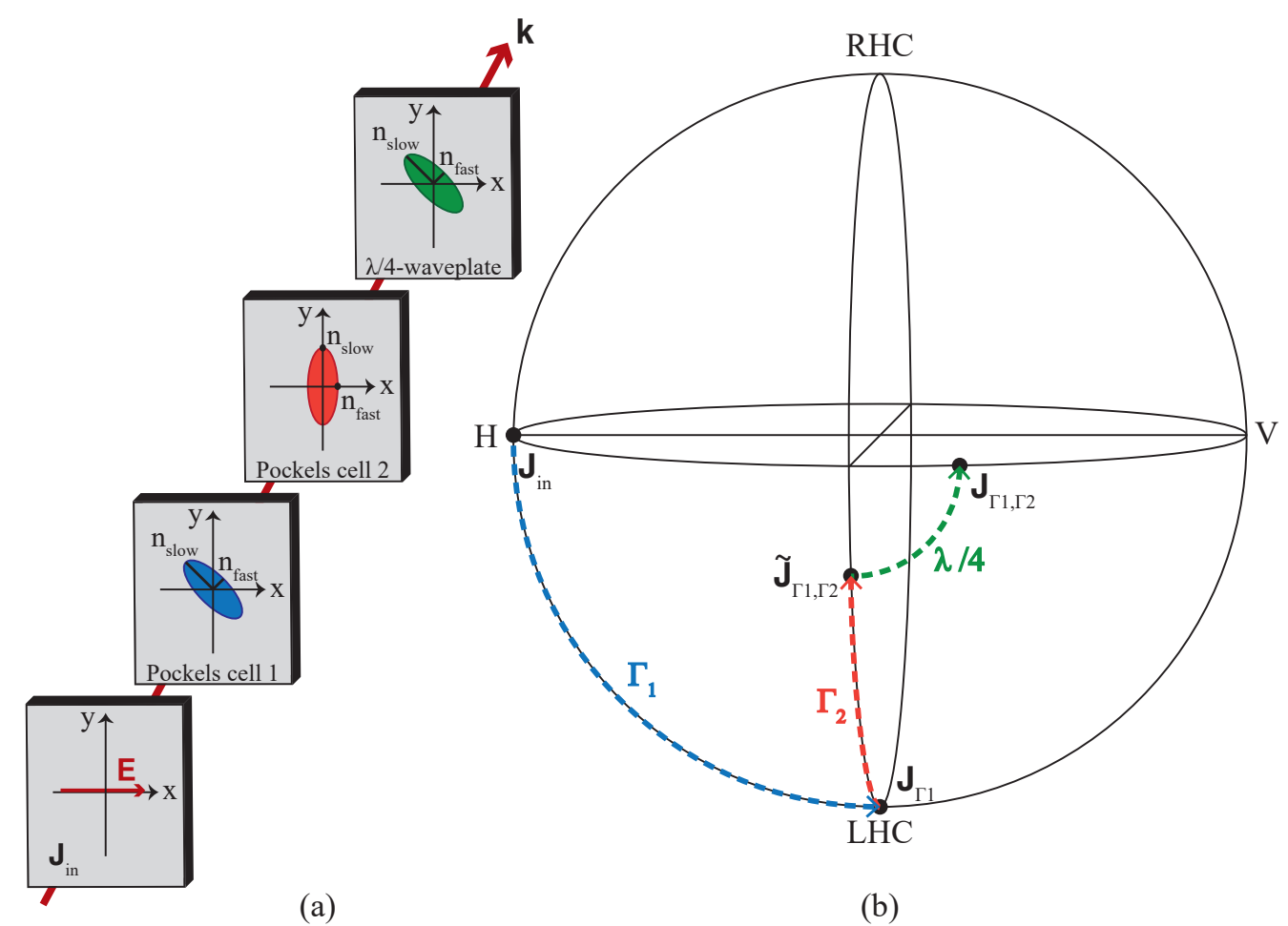

Figure 3.7: Illustration of the polarization state generator's working principle. (a) The electric field vector of the incident beam as well as the index ellipsoids for the three components - two Pockels cells and a quarter-wave plate - of the polarization state generator are graphically displayed. (b) The polarization states at different stages throughout the transformation are visualized on the Poincaré sphere. Linear polarization states can be found along the equator of the sphere, including the initial horizontal polarization $\boldsymbol{J}_{\text {in }}$, indicated by $\mathrm{H}$, as well as vertical polarization $\mathrm{V}$ on the opposite site of the sphere. The poles represent circular polarization states, namely right-handed circular (RHC) and lefthanded circular (LHC) polarization. The transformation by the first Pockels cell with the phase shift $\Gamma_{1}$ is depicted in blue, whereas the effect of the second Pockels cell, with $\widetilde{\boldsymbol{J}}_{\Gamma_{1}, \Gamma_{2}}$ as resulting polarization state, is visualized in red. The subsequent quarter-wave plate is indicated in green alongside with the final linear polarization state $\boldsymbol{J}_{\Gamma_{1}, \Gamma_{2}}$ (modified in accordance to [Krü17]). 
As can be seen from the complex $y$-component, the first Pockels cell affects the ellipticity. Inserting the components of $\boldsymbol{J}_{\Gamma_{1}}$ in equations (2.16) and 2.17) indicates that the ellipticity angle changes to $\chi=\frac{\Gamma_{1}}{2}$, whereas the polarization angle is $\Psi=0$ due to the induced phase shift of $\frac{\pi}{2}$ between the electric field components. Considering the Poincaré sphere as depicted in figure 3.7. the polarization change introduced by the first Pockels cell can be described by a transformation along the meridian. The phase retardation of the first Pockels cell is in the following set to $\Gamma_{1}=\frac{\pi}{2}$, yielding circular polarization for the emergent beam.

The phase retardation $\Gamma_{2}$ of the second Pockels cell is kept variable. The effect on the emergent beam is described by

$$
\widetilde{\boldsymbol{J}}_{\pi / 2, \Gamma_{2}}=\left(\begin{array}{cc}
e^{i \frac{\Gamma_{2}}{2}} & 0 \\
0 & e^{-i \frac{\Gamma_{2}}{2}}
\end{array}\right) \frac{1}{\sqrt{2}}\left(\begin{array}{c}
1 \\
i
\end{array}\right)=\frac{1}{\sqrt{2}}\left(\begin{array}{c}
e^{i \frac{\Gamma_{2}}{2}} \\
i e^{-i \frac{\Gamma_{2}}{2}}
\end{array}\right)
$$

yielding a polarization angle of $\Psi=45^{\circ}$ and an ellipticity of $\chi=\frac{\pi}{4}-\frac{\Gamma_{2}}{2}$ when applying equations (2.16) and 2.17). In terms of the Poincaré visualization, the influence of the second Pockels cell can be described by a transformation along a meridian rotated by $90^{\circ}$ compared to the meridian for the transformation induced by the first Pockels cell.

The ellipticity of the output wave after the Pockels cells pair is determined by the phase retardation $\Gamma_{2}$. Contrary, the beam's polarization angle $\Psi$ is fixed to $45^{\circ}$, which does not yet meet the requirement of accessing all linear polarization states, i.e. arbitrary $\Psi$ for $\chi=0$. Therefore, a quarter-wave plate is added, which introduces a phase shift on the beam described by

$$
\begin{aligned}
\boldsymbol{J}_{\pi / 2, \Gamma_{2}} & =\left(\begin{array}{cc}
\cos \left(\frac{\pi}{4}\right) & i \sin \left(\frac{\pi}{4}\right) \\
i \sin \left(\frac{\pi}{4}\right) & \cos \left(\frac{\pi}{4}\right)
\end{array}\right) \frac{1}{\sqrt{2}}\left(\begin{array}{c}
e^{i \frac{\Gamma_{2}}{2}} \\
i e^{-i \frac{\Gamma_{2}}{2}}
\end{array}\right) \\
& =i\left(\begin{array}{c}
\sin \left(\frac{\Gamma_{2}}{2}\right) \\
\cos \left(\frac{\Gamma_{2}}{2}\right)
\end{array}\right)=e^{i \frac{\pi}{2}}\left(\begin{array}{c}
\cos \left(\frac{\pi}{2}-\frac{\Gamma_{2}}{2}\right) \\
\sin \left(\frac{\pi}{2}-\frac{\Gamma_{2}}{2}\right)
\end{array}\right) .
\end{aligned}
$$

Hence, the output wave after the polarization state generator is characterized by a polarization angle of $\Psi=\frac{\pi}{2}-\frac{\Gamma_{2}}{2}$. This implies that the polarization orientation is defined by the phase retardation imprinted by the second Pockels cell. The ellipticity amounts to 0 , since there is no phase difference between the two electric field components. The pre-factor of $e^{i \frac{\pi}{2}}$ indicates a phase retardation of $\frac{\pi}{2}$ of the whole beam with respect to the incident beam. Visualizing the effect of the quarter-wave plate on the Poincare sphere yields a transformation along a meridian parallel to the one indicating the transformation induced by the first Pockels cell (see figure 3.7). By changing the applied voltage and hence retardation of the second Pockels cell, an arbitrary linear polarization orientation of the emergent beam can be achieved. Contrary, a constant retardation of $\frac{\pi}{2}$ is applied to the first Pockels cell, which hence acts as a quarter-wave plate and could in an ideal configuration be replaced thereby. Experimentally, 
polarization sensitive components like lenses and dichroic mirrors introduce distortions to the polarization state, resulting in a change of the ellipticity. The first Pockels cell allows for the flexibility to nevertheless achieve any desired polarization state and is thus utilized to compensate for such distortions.

Exemplary calibration curves for the two Pockels cells in the polarization state generator are shown in figure 3.8. They are determined with the help of a polarimeter (Schäfter + Kirchhoff $\mathrm{GmbH}$, Germany) via a self-written LabVIEW routine. The ellipticity $\chi$, defined by equation (2.17), serves as the measure for linearity, i.e. $\chi=0$ corresponds to linearly polarized light. It is recorded by the polarimeter, which is placed in the beam path behind the quarterwave plate of the polarization state generator. For a chosen voltage $U_{2}$ applied to the second Pockels cell, the voltage applied to the first Pockels cell is altered until a linear polarization state is recorded by the polarimeter. The upper threshold for the ellipticity is chosen to $\chi=0.005$, corresponding to an ellipticity angle of $\chi=0.3^{\circ}$ and thus yielding an undistorted depletion pattern according to [Krü17]. The voltage $U_{1}$ is displayed in figure 3.8(a) and is nearly constant, compensating only for misalignments of the system as well as distortions introduced by optical components.

Figure 3.8(b) shows the expected linear dependence between the voltage $U_{2}$ applied to the second Pockels cell, which is directly related to the induced phase shift $\Gamma_{2}$ following equation (2.39), and the resulting polarization angle $\Psi$. Contrary to the example curves shown in Vin15] and [Krü17, all linear polarization states, i.e. the total range $\Psi \in\left[0,180^{\circ}\right]$, are accessible with the configuration of the polarization state generator described herein. Moreover,

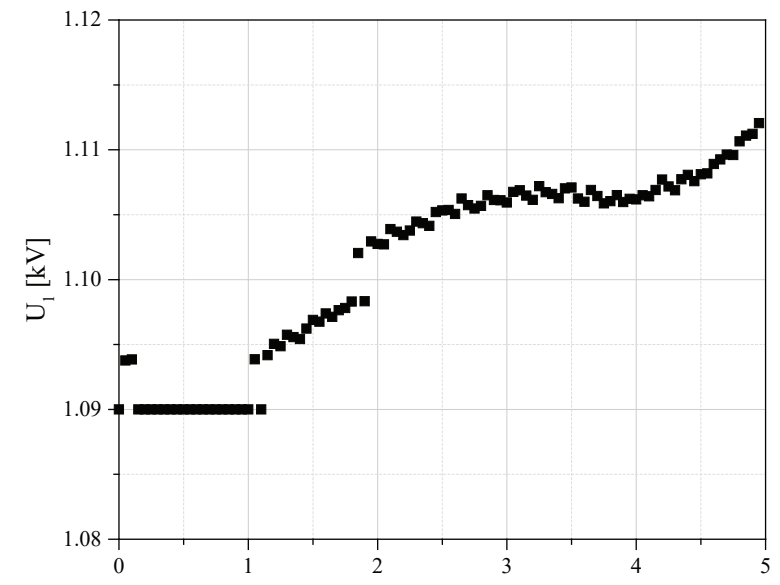

(a)

$\mathrm{U}_{2}[\mathrm{kV}]$

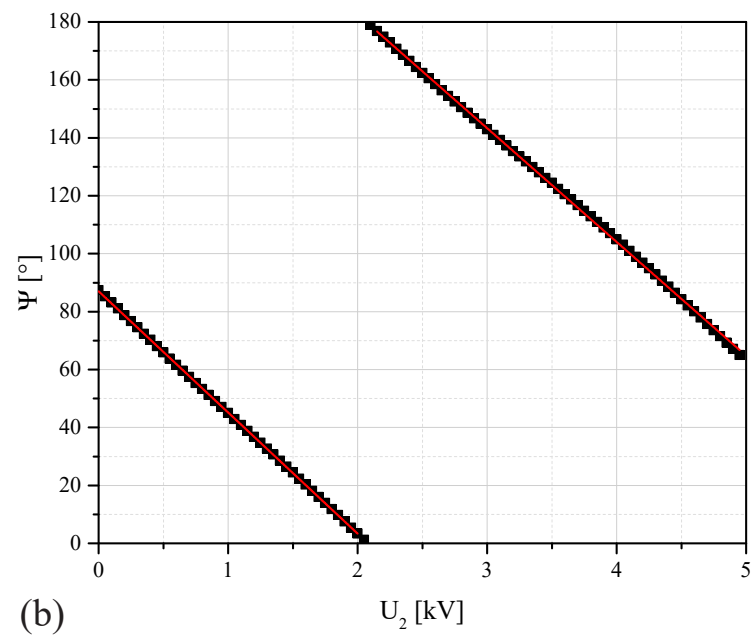

(b)

Figure 3.8: Exemplary calibration curves for the Pockels cells of the polarization state generator. (a) Voltage $U_{1}$ applied to the first Pockels cell as a function of the voltage $U_{2}$ applied to the second Pockels cell. (b) Angle of the output wave's polarization direction $\Psi$ as a function of the voltage $U_{2}$ applied to the second Pockels cell. A linear fit outlines the dependence between $\Psi$ and $U_{2}$. 
the fluctuations of $U_{1}$ are much smaller compared to the previously presented results. These advantages originate from the additional quarter-wave plate.

\section{Polarization State Analyzer}

In the last subsection, the generation of a linear polarization with arbitrary orientation by means of the Polarization State Generator has been demonstrated. For the depletion pattern generation, not only the linear polarization of the incident beam undergoing conical diffraction is of utter importance, but also the subsequent filtering of beam components in the diffracted beam. This is conducted by the Polarization State Analyzer as described in the following.

A beam with an incident linear polarization with orientation $\alpha$, i.e.

$$
\boldsymbol{J}_{\text {in }}=\left(\begin{array}{c}
\cos \alpha \\
\sin \alpha
\end{array}\right)
$$

passes a series of a quarter-wave plate and two Pockels cells as described in section 3.2 . The Pockels cells' fast axes enclose an angle of $0^{\circ}$ and $45^{\circ}$ with the $x$-axis, respectively. The quarterwave plate is oriented in a $45^{\circ}$ position similar to the arrangement of the Polarization State Generator. This results in a polarization vector of

$$
\boldsymbol{J}_{\Gamma_{3}, \Gamma_{4}}=\boldsymbol{T}_{\Gamma_{4}}\left(45^{\circ}\right) \boldsymbol{T}_{\Gamma_{3}}\left(0^{\circ}\right) \boldsymbol{T}_{\lambda / 4}\left(45^{\circ}\right) \boldsymbol{J}_{\mathrm{in}}
$$

of the emergent beam. Assuming a constant retardation of $\Gamma_{4}=\frac{\pi}{2}$ imprinted by the second Pockels cell yields

$$
\boldsymbol{J}_{\Gamma_{3}, \pi / 2}=e^{i \frac{\pi}{2}}\left(\begin{array}{c}
\cos \left(\frac{\pi}{2}-\frac{\Gamma_{3}}{2}-\alpha\right) \\
\sin \left(\frac{\pi}{2}-\frac{\Gamma_{3}}{2}-\alpha\right)
\end{array}\right) .
$$

The prefactor $e^{i \frac{\pi}{2}}$ indicates a phase shift of the whole beam with respect to the incoming beam. The emergent beam is linearly polarized, with the polarization angle given by $\Psi=\frac{\pi}{2}-\frac{\Gamma_{3}}{2}-\alpha$. This implies that the polarization angle of incident and emergent beam are linearly related and thus for fixed phase retardation $\Gamma_{3}$ assigned unambiguously. A Glan-Thompson prism with fixed transmission axis along $\Psi_{\text {trans. }}=\frac{\pi}{2}$, placed behind the Pockels cells pair, can hence be used for filtering of undesired beam components: For transmitting components with initial polarization orientation $\widetilde{\Psi}$ through the complete polarization state analyzer, the retardation is chosen to be $\Gamma_{3}=-2 \widetilde{\Psi}$. The desired beam components exhibit a polarization orientation of $\frac{\pi}{2}$ after the Pockels cells and are thus transmitted by the Glan-Thompson prism.

The alignment of the Polarization State Analyzer is, similarly to the calibration of the Polarization State Generator, realized by a self-written LabVIEW routine. To simplify the align- 
ment procedure, a thin polarizing foil with known transmission direction is placed before the Polarization State Analyzer. Via a rotation mount, different angles of the incident beam's polarization orientation can then be realized. For each angle, the voltages for the two Pockels cells are determined such that the emergent beam's polarization, which is controlled with the polarimeter, is linearly vertical. The resulting calibration curves are similar to the ones displayed in figure 3.8 .

Depending on the desired depletion pattern orientation, the transmission direction and thus the retardation are chosen as described in section 3.2 . The polarization of the emergent beam is determined by the transmission direction of the Glan-Thompson prism and is thus fixed. In order to match the polarization direction in the back focal plane with the depletion pattern orientation, the final adjustment of the polarization direction is conducted by a third pair of Pockels cells. Its working principle is analog to the Polarization State Generator, i.e. any chosen linear polarization direction for the emergent beam can be realized for a fixed polarization of the incident beam.

\section{Switching frequency}

There are two main parameters influencing the switching speed achievable with the setup presented herein: the optical rise time of the Pockels cells and the rise time of the high-voltage amplifiers employed. The first is in the range of a few nanoseconds and is thus negligible. However, the rise time of the amplifiers needs to be considered.

For tomoSTED microscopy, a rotation of the polarization direction by $180^{\circ}$, i.e. a change of $\Psi$ by $\pi$, needs to be realized by an appropriate voltage step. The Pockels cells employed consist of $\mathrm{KD}^{*} \mathrm{P}$, which exhibits a Pockels coefficient of $\mathfrak{r}_{\mathrm{KD}}{ }_{\mathrm{P}}=25 \mathrm{pm} / \mathrm{V}$ [GAM]. According to the manufacturer's specifications, the half-wave voltage, i.e. the voltage necessary for a phase retardation of $\pi$, is $U_{\pi, \lambda_{0}=1060 \mathrm{~nm}} \approx 3 \mathrm{kV}$ for $\lambda_{0}=1060 \mathrm{~nm}$. Following equation (2.38), $U_{\pi}$ depends linearly on the wavelength when neglecting dispersion effects, leading to an expected half-wave voltage of $U_{\pi, \lambda_{0}=775 \mathrm{~nm}} \approx 2.2 \mathrm{kV}$ for the STED wavelength. As the expected polarization angle is given by $\Psi=\frac{\pi}{2}-\frac{\Gamma_{2}}{2}$ with $\Gamma_{2}$ denoting the retardation induced by the second Pockels cell of the Polarization State Generator, a retardation of $\pi$ corresponds to a change of $\frac{\pi}{2}$ of the polarization direction. A half-wave voltage of around $U_{\pi \text {,meas. }} \approx 2.2 \mathrm{kV}$ can actually be deduced from figure 3.8 (b), coinciding with the expected value. Also the voltage $U_{1}$ applied to the first Pockels cell in order to achieve a $\lambda / 4$ retardation is in very good agreement with these observations.

For the experimental implementation presented within this work, high voltage amplifiers with a voltage change of $3 \mathrm{kV}$ in $2.3 \mu \mathrm{s}$ are utilized to control the Pockels cells. For a full rotation of the pattern orientation, corresponding to a voltage change of $2 U_{\pi, \lambda_{0}=775 \mathrm{~nm}} \approx 4.4 \mathrm{kV}$, this 
yields an upper bound of $\approx 300 \mathrm{kHz}$ to the switching frequency.

Hence, the switching frequency for a pattern generated by conical diffraction is approximately three orders of magnitude higher compared to a pattern generated with an SLM, enabling the desired pixel-wise switching of the pattern orientation.

\subsection{Reconstruction algorithms}

With the methods discussed beforehand, a 1D depletion pattern with switchable orientation can be generated and employed for the acquisition of individual sub-images under $N$ different pattern orientations. These sub-images need to undergo a subsequent image processing step in order to obtain an, in terms of resolution, sufficiently isotropic estimation of the unknown sample. In this section, two reconstruction approaches, introduced and evaluated for tomoSTED microscopy in [Krü17, are summarized and their respective advantages are highlighted.

Regardless of the chosen reconstruction method, a correction for oversampling along the confocal axis, as outlined in the following, compensates for a higher noise level as compared to an ideal sampling and is implemented as additional image processing step prior to the reconstruction. All reconstruction algorithms were implemented in MATLAB by J. Keller-Findeisen and J.-R. Krüger as described in detail in [Krü17.

\subsubsection{Noise correction for oversampling}

The choice of the pixel size as a parameter for the image acquisition is crucial for the observable resolution as well as for the signal-to-noise ratio (SNR). An ideal pixelation obeys Nyquist's sampling theorem in order to transfer all spatial frequencies and hence resolution information from the underlying continuous structure to the finitely sampled image. Contrary, the sampling for realistic imaging conditions, i.e. in the presence of noise, should be as coarse as possible to maximize the SNR assuming a constant overall imaging time. For a 1D depletion pattern, the optimum sampling is direction-dependent due to the non-isotropic expected resolution enhancement, resulting in a complex sampling grid which would need to be adjusted dependent on the chosen depletion pattern orientation.

To simplify the experimental implementation, a sampling grid with squared pixels is chosen in compliance with the super-resolved axis, resulting in a highly oversampled image in the direction of the confocal axis. This oversampling can be corrected in order to obtain the same SNR as in the ideal sampling case, as outlined in the following.

Theoretically, an adequate correction of the noise level is sufficient to compensate for the oversampling, as explained in detail in [Krü17. As this is not feasible experimentally, a filter array is employed to join an adequately chosen number of pixels along the confocal direction to one single pixel. The size and entries of this filter array are solely dependent on the oversampling factor, i.e. the resolution enhancement $k$. An artificial enhancement of signal is avoided 
by ensuring that the totality of the entries of the filter array amounts to 1 . For $k=5$ and a depletion pattern orientation of $0^{\circ}$, i.e. a vertically oriented high-resolution direction, the filter is thus given by [Krü17]

$$
\left(\begin{array}{ccccc}
0 & 0 & 0 & 0 & 0 \\
0 & 0 & 0 & 0 & 0 \\
\frac{1}{5} & \frac{1}{5} & \frac{1}{5} & \frac{1}{5} & \frac{1}{5} \\
0 & 0 & 0 & 0 & 0 \\
0 & 0 & 0 & 0 & 0
\end{array}\right)
$$

To match the direction of the confocal axis, a rotation matrix is multiplied with the filter array, and the respective sub-image is convolved with the associated resulting filter [Krü17].

\subsubsection{Maximum-value reconstruction}

The individual sub-images for the different pattern orientations, after being corrected for oversampling, need to be combined to reconstruct a final image with isotropic resolution enhancement. Theoretically, the best image assembly takes only the high-resolution information from each sub-image into account, reducing their OTFs essentially to the axis containing the highest frequencies and thus the highest resolution information (cf. subsection 2.2.2). This can be achieved by comparing all OTFs of the individual sub-images for every frequency coordinate and keeping the maximum value for an assembled OTF: Only the largest contribution to each spatial frequency is preserved, corresponding to the high-resolution information. The inverse Fourier transform of the assembled OTF subsequently yields the final reconstructed image (cf. figure 3.9 ).

For a continuous rotation of the pattern, the assembled OTF equals the in subsection 2.2.2

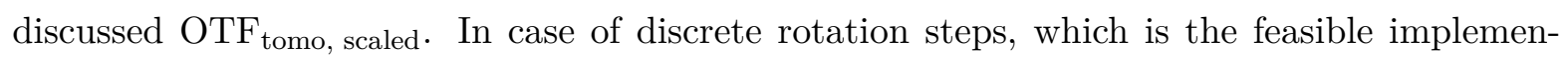
tation, the assembled OTF is not fully homogeneous, but rather features a "star-like" shape Krü17. This has however been shown of having no visible effect on the assembled image for an appropriately chosen number of pattern orientations Krü17.

Comparing the signal of the reconstructed image with that of the sub-images allows one main observation. By keeping the maximum value of all OTFs at all frequency coordinates, the resolution is increased and the signal is narrowed down to a smaller spot. Since the overall fluorescence signal contained in the amplitude of the OTF at $u=v=0$ is approximately equal for all sub-images and thus directly transferred to the assembled OTF, the peak intensity in the reconstruction is thus higher. This higher maximum intensity in the reconstructed image compared to the raw sub-images can also be seen in figure 3.9. This observation, meaning the conservation of signal, is of particular importance in realistic imaging conditions, i.e. for a low SNR [Krü17]. 
Overall, for an appropriately chosen number of pattern orientations, the 1D resolution enhancement can be preserved without introducing artifacts at a high signal level [Krü17, as also seen from figure 3.9. Moreover, the method does not require any prior knowledge of the optical system like the pattern directions or the resolution enhancement, since it forgoes a preceding

(a)

Reconstruction
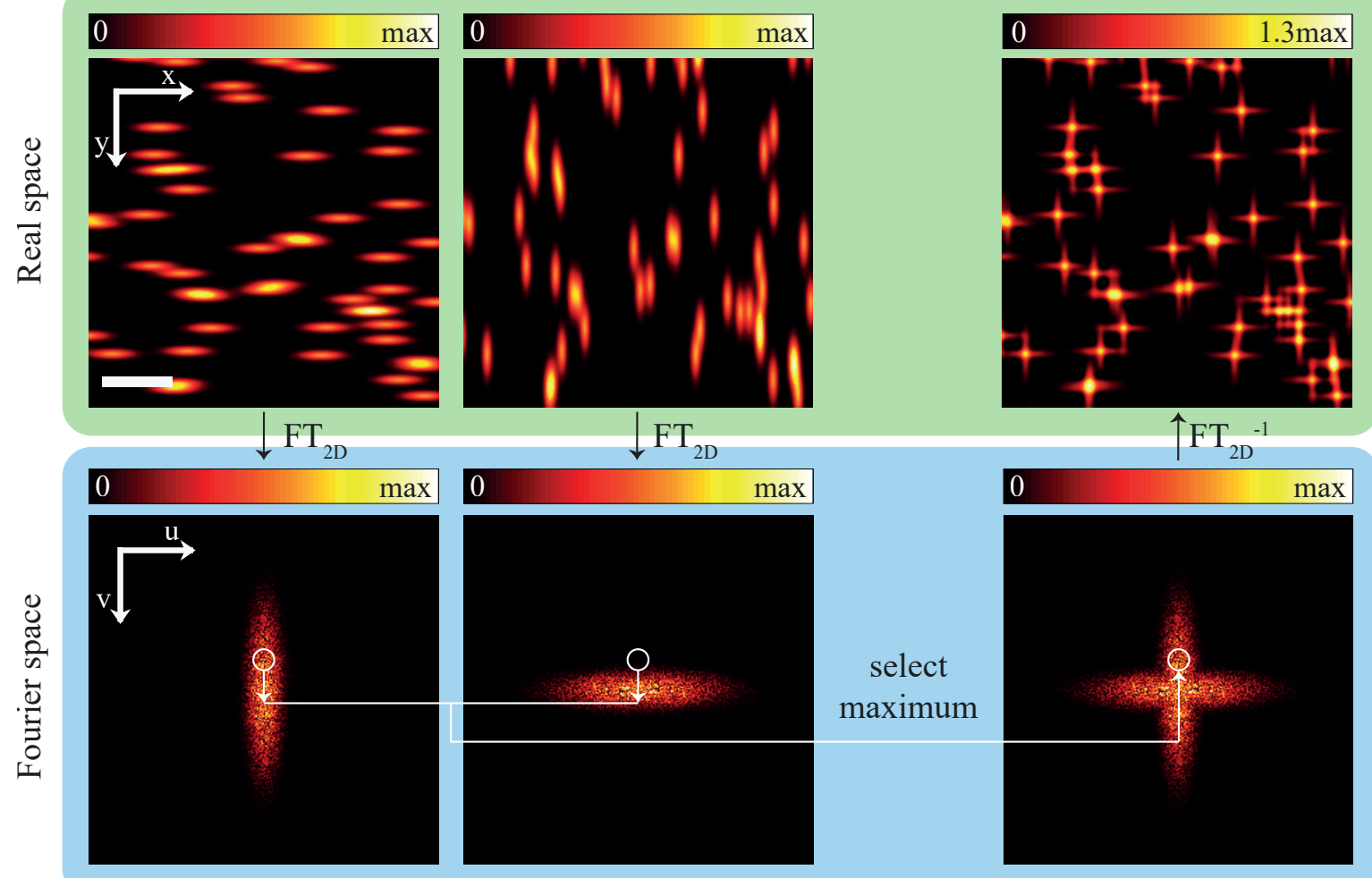

(b)

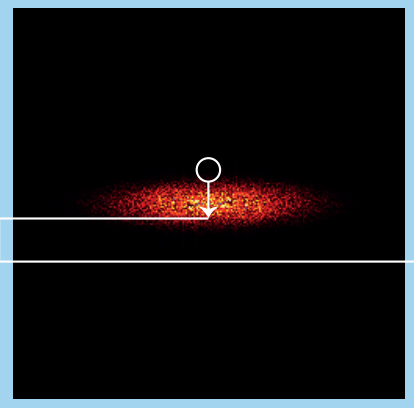

(c)
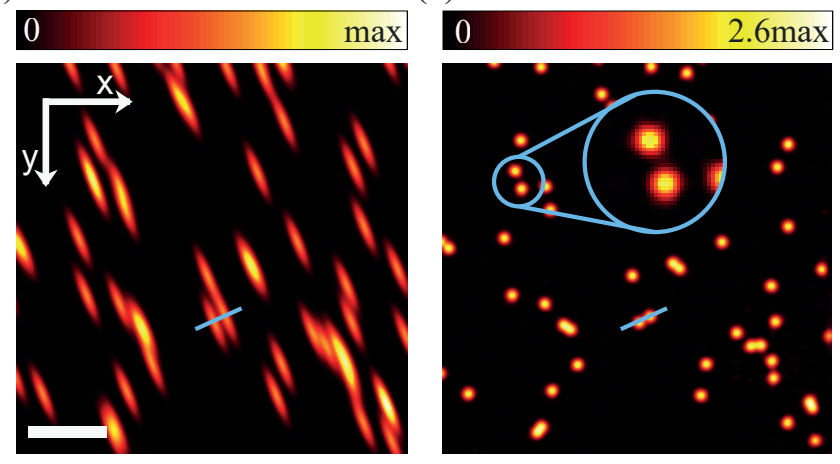

(d)

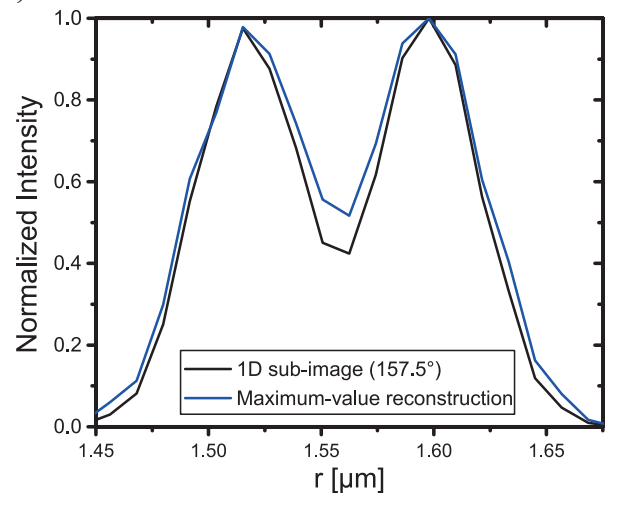

Figure 3.9: Principle of the maximum-value reconstruction (taken from Krü17]): (a) Demonstration of the basic idea on simulated images for two depletion pattern orientations. The images are transformed to Fourier space, the maximum value for each pixel is kept and the assembled image is backtransformed to real space. (c) TomoSTED image for $N=8$ pattern orientations, obtained by maximum-value reconstruction, and (b) one exemplary sub-image showing a 1D resolution increase. The blue circle highlights an exemplary area, showing no artifacts for the reconstruction. (e) Intensity plots along line profiles highlighted in (b,c). All scale bars indicate a length of $0.5 \mu \mathrm{m}$. 
determination of the PSF.

Nevertheless as noted in subsection 2.2.2 the signal per sub-image scales with $k / N$ compared to the 2D STED image for the same total acquisition time and the same resolution enhancement $k=k_{1 \mathrm{D}}=k_{2 \mathrm{D}}$. Here, $N$ denotes the number of pattern orientations chosen for the acquisition. For challenging imaging conditions with a low SNR, it might thus be appealing to decrease $N$ even below $N_{\text {opt }}$ as introduced in subsection 2.2.2. As demonstrated in [Krü17], a choice of $N=N_{\text {opt }} / 2$ can still be adequate under certain circumstances, but ray-like artifacts and a decrease of the resolution enhancement are unavoidable. The choice of $N$ has therefore to be seen as a trade-off between a sufficient sampling of pattern orientations or an enhanced signal at the price of a lower resolution enhancement. A reconstruction approach dealing well also with less pattern orientations is the so-called Richardson-Lucy deconvolution, which is presented in the following.

\subsubsection{Richardson-Lucy deconvolution}

The Richardson-Lucy deconvolution is an iterative deconvolution procedure with the aim of approximating an unknown object $W$ by means of probability estimates. The basic idea was first presented by [Ric72] as a method for the restoration of noisy degraded images and by [Luc74] as a technique for generating estimates to solutions of deconvolution problems in statistical astronomy. It is summarized subsequently, following the notation introduced in [Krü17].

The imaging process can be mathematically described by the convolution of the real, generally unknown, object $W$ and the PSF $h_{j}$ of the imaging system. For tomoSTED microscopy, $h_{j}, j \in\{1, \ldots, N\}$ equals the PSF for each depletion pattern orientation, with $N$ being the number of recorded orientations. The obtainable sub-image $G_{j}$ for direction $j$ can thus be expressed as

$$
G_{j}=W * h_{j}
$$

The Richardson-Lucy deconvolution exploits a-priori knowledge about the PSF of the imaging system and the information of the recorded image in order to find an estimation of the object. The technique is based on Bayes' theorem on conditional probability and aims on maximizing the likelihood of the observed sample, conserving constraints on the frequency distribution, i.e. normalization and non-negativity [Luc74]. Contrary to the maximum-value reconstruction, the Richardson-Lucy method has the significant advantage of exploiting the overall fluorescence signal for the deconvolution procedure.

Starting with an initial estimate $W_{\text {est }}^{0}$ for the object, which is of the same size as the detected image, i.e. an array of ones, the Richardson-Lucy result $F_{j}^{i+1}$ for the sub-image $j$, 
$j \in\{1, \ldots, N\}$, and the $(i+1)$-th step is given by [Krü17]

$$
F_{j}^{i+1}=\frac{G_{j}}{G_{\mathrm{est}, j}^{i}} \star h_{j}
$$

with $G_{\mathrm{est}, j}^{i}=W_{\mathrm{est}}^{i} * h_{j}$ denoting the estimated sub-image in step $i$ and $\star$ the $2 \mathrm{D}$ correlation. This estimated sub-image is compared with the real image $G_{j}$ in order to obtain a weighting array $\frac{G_{j}}{G_{\text {est }, j}^{i}}$ included in the Richardson-Lucy result. The overall Richardson-Lucy result for the $(i+1)$-th step is given by the sum of the results for the sub-images [Ric72, i.e.

$$
F^{i+1}=\sum_{j} F_{j}^{i+1}
$$

and corresponds to a weighting image, which is subsequently multiplied with the estimated object to yield an improved estimate

$$
W_{\mathrm{est}}^{i+1}=W_{\mathrm{est}}^{i} \cdot F^{i+1}
$$

In realistic imaging conditions, i.e. for strongly varying fluorescence signal levels and in the presence of noise [Krü17, low fluorescence signals are not well conserved, especially if arising from areas in proximity of high fluorescence signal regions. This enhancement of contrast, resulting in a loss of information, can be counteracted by an additional regularization as derived by [CM96]. After each iteration step, an intensity regularization

$$
W_{\mathrm{est}, \mathrm{reg}}^{i+1}=\frac{-1+\sqrt{1+2 \cdot \alpha_{R c h} \cdot W_{\mathrm{est}}^{i+1}}}{\alpha_{R c h}}
$$

is performed on the updated estimate of the object, with $\alpha_{R c h}$ denoting the regularization parameter.

As for the maximum-value reconstruction, the choice of the number of pattern orientations is a trade-off between sufficient sampling and a high signal in the individual sub-images. A characterization of the necessary number of pattern orientations for Richardson-Lucy deconvolution has been performed in [Krü17] by taking radial OTF profiles for the estimated images for different numbers of iteration steps (see figure 3.10). The radius for the profile was chosen corresponding to the high-resolution FWHM in real space. For the number of pattern orientations chosen as $N_{\text {opt }} / 2$, an oscillatory behavior due to the incomplete sampling of the Fourier space is observable. The averaged intensity at the local minima has been shown to reach $60 \%$ of the maximal intensity if the number of iteration steps is chosen sufficiently high Krü17. This compares to approximately $10 \%$ for the same quantity in case of the maximum-value reconstruction (cf. figure 3.10), highlighting the better performance of the Richardson-Lucy deconvolution in case of a low number of pattern orientations. 

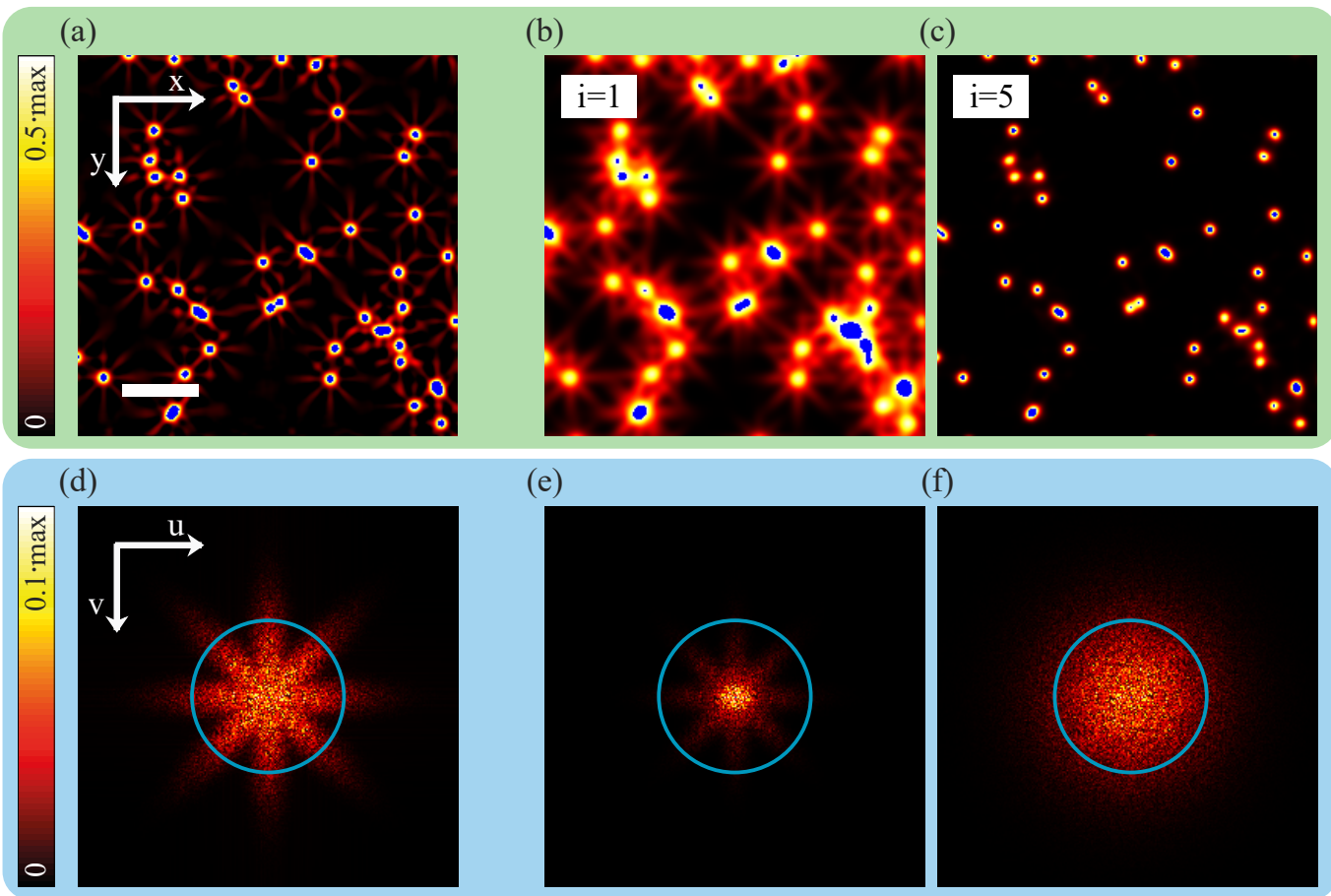

(e)

(f)

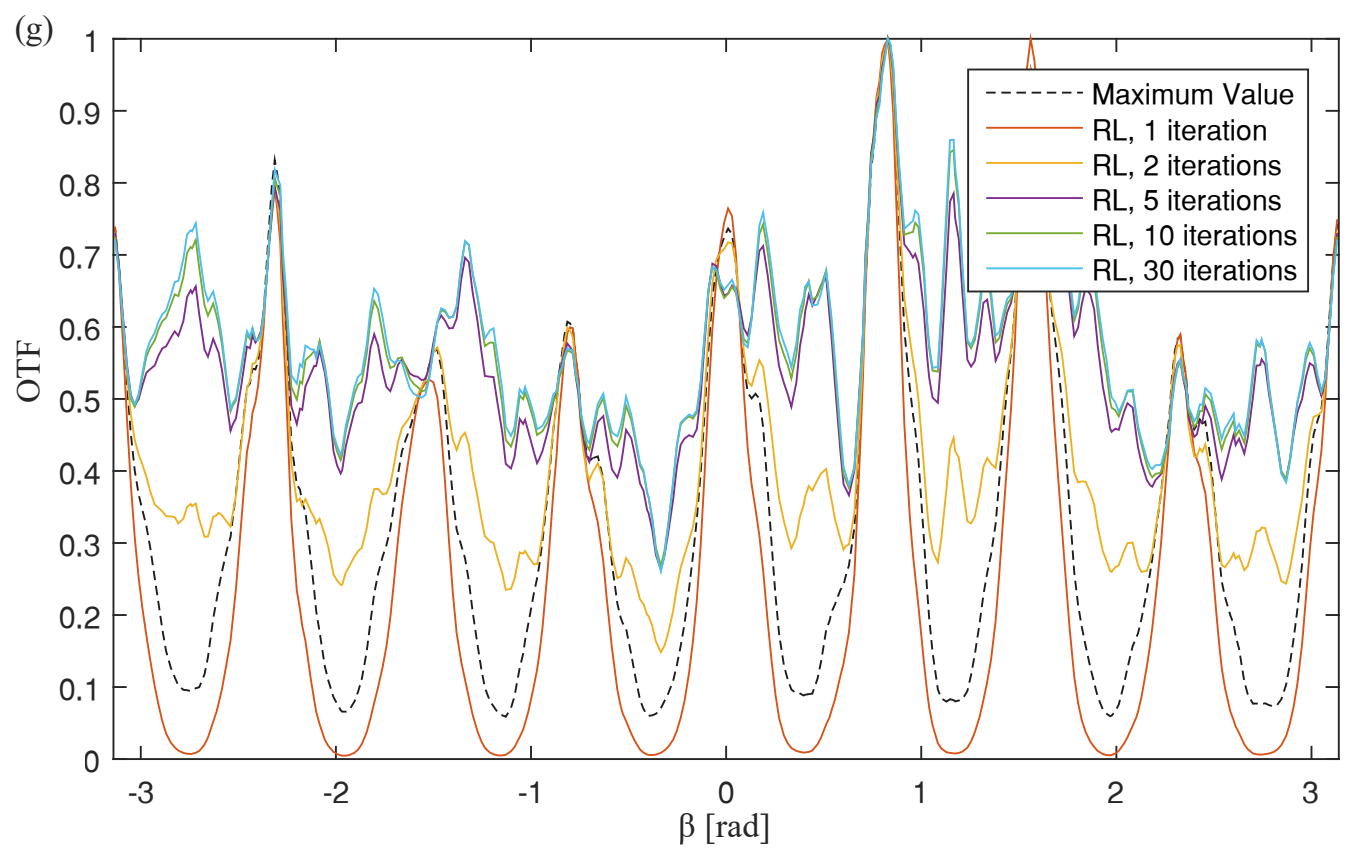

Figure 3.10: Principle of the Richardson-Lucy deconvolution (taken from [Krü17]): Simulated data for a resolution enhancement of $k_{1 \mathrm{D}}=5$ and $N=4$ depletion pattern orientations are reconstructed by (a) the maximum-value reconstruction and (b,c) the Richardson-Lucy deconvolution $\left(\alpha_{R c h}=0\right)$ for one and five iteration steps, respectively. The scale bar indicates a length of $0.5 \mu \mathrm{m}$ and the intensity scale is chosen such that the ray-like artifacts in (a) and (b) are clearly visible. (d,e,f) are the respective Fourier transforms of $(\mathrm{a}, \mathrm{b}, \mathrm{c})$, where the insufficient sampling in case (a) is clearly visible. (g) Normalized intensity profiles along a circle of radius $\frac{1}{\Delta_{S T E D}}$ (indicated in blue in $(\mathrm{d}, \mathrm{e}, \mathrm{f})$ ) show the increase in the averaged intensity at the local minima for an increasing number of iteration steps. 


\subsection{Adaptive scanning}

So far, tomoSTED microscopy has been discussed in the context of a fast imaging technique for acquiring a two-dimensional image with a pixel-wise pattern rotation. However, it is by far not limited to this: Pixel-wise pattern rotation opens the path to a variety of applications, including adaptive scan patterns for filament tracing. Adaptive scan patterns can also be employed for improving the performance of 2D STED microscopy, as the introduction of the novel technique FastRESCue reveals. The experimental requirements for both techniques, including a novel scan system, are discussed herein.

FastRESCue Conventional RESCue STED microscopy [SER ${ }^{+} 11$ ] (see also section 2.3) allows to reduce the light dose imposed on a sample by switching both the excitation and STED laser off for the residual pixel time if one of two conditions is met:

- Enough signal has been collected (adjustable upper threshold) or

- no signal arises in the first e.g. $10 \%$ of the pixel dwell time (adjustable lower threshold).

This is typically experimentally realized by employing acousto-optic modulators, which provide switching times in the range of around $200 \mathrm{~ns}$ and thus enable the switching of laser states significantly faster than the pixel dwell time.

In this way an up to fivefold reduction of the light dose [ $\left.\mathrm{HRH}^{+} 17\right]$ or eightfold bleaching reduction $\left[\mathrm{SER}^{+} 11\right]$ compared to a conventional pulsed STED microscope could be demonstrated, depending on the sparsity of the sample. On the contrary, this implies that $80 \%$ of the total acquisition time is spent without recording signal waiting for the scan to continue. This aspect of RESCue is refined by not turning the lasers off for the remaining pixel dwell time, but moving to the next pixel immediately as also suggested in [SER ${ }^{+} 11$, a technique referred to as FastRESCue in the following. A similar technique has been shown for RESOLFT nanoscopy recently [ $\left.\mathrm{DCC}^{+} 19\right]$, yielding a significant speed-up of the acquisition time. However, the pixel dwell times and hence the probing times for RESOLFT are in the order of several hundreds and tens of microseconds, respectively, simplifying the experimental implementation drastically. The proposed FastRESCue technique poses significant challenges on the scanning device, as it implies an almost-immediate response to the signal count and a varying pixel dwell time and thus makes the galvanometer scanner utilized in $\left[\overline{\mathrm{DCC}^{+} 19}\right]$ an unsuitable choice.

Filament tracing In live cells, filaments move within the typical time of an image acquisition as highlighted in section 1. To temporally resolve such dynamics, the total acquisition time needs to be reduced by a reduction of either the pixel dwell time or the field of view. FastRESCue as described before is a way to reduce the acquisition time without compromising on the amount of signal collected. For a further reduction of acquisition time, it is necessary to limit the field of view. The most efficient way of scan field reduction is obviously to image 
only a narrow band around the filament of interest. This poses the problem of determining the accurate positioning of this band: For a living cell, the filament will move over time, eventually moving even out of the initial field of view. Thus, a constant adaption of the region of interest is necessary, controlled by the results of the previous scan. A fast decision for a scan position as well as a scanning device with a modifiable scan pattern and fast response is inevitable.

The requirements for the scan system for an experimental realization of these techniques, namely FastRESCue and filament tracing, can hence be summarized as follows:

- response time at least in the range of the typical pixel dwell time, i.e. some microseconds

- variable scan speed

- flexible positioning control, i.e. no pre-defined scan patterns

- precise positioning in the range of a typical structure size, i.e. some nanometers

- achromatic deflection in the range from $\lambda_{0}=649 \mathrm{~nm}-775 \mathrm{~nm}$

- several tens of micrometer scan range

The galvanometer scanner in combination with the acquisition software utilized so far does not fulfill these requirements, as explained in the following.

\subsubsection{Galvanometer scanner}

The QUAD beamscanner uses four mirror galvanometers, driven by analog voltages, to move the beam across the sample. The four mirrors are arranged in two pairs, each of which is utilized to deflect the beam along one of the two scan axes, ensuring that no offset in the back-focal plane is introduced across the full scan range [BREH11]. Therefore, the positioning of the two paired mirrors relative to each other and hence also the applied voltages are interdependent. An exemplary snippet of the voltage courses over time applied to the scanner is displayed in figure 3.11 for all four mirrors under typical acquisition conditions. For a classical image scan, a triangular shaped signal is applied to the scanner's mirrors. Studying exemplarily the $x$-axis, which is usually the fast scan axis corresponding to a line in the image, and in particular $X 1$, the graph can be split in areas of rising and falling voltages with the latter being comparably short. Areas of continuously increasing voltage resemble the time of a line acquisition, with the signal collected by the detector during the scan being assigned to the corresponding pixel. The falling segments are used to move the scanner back to the starting position to acquire a new line. The $y$-direction is handled accordingly with a much smaller slope for the voltage increase, corresponding to one scan cycle per image. 
The dependence between $\mathrm{X} 1$ and $\mathrm{X} 2$ or $\mathrm{Y} 1$ and $\mathrm{Y} 2$ is of linear nature and is determined as

$$
\begin{aligned}
& U_{X 2}=-0.960 \cdot U_{X 1}-0.463 \mathrm{~V}, \\
& U_{Y 2}=-0.960 \cdot U_{Y 1}+0.07 \mathrm{~V} .
\end{aligned}
$$

In the following, the mirror $\mathrm{X} 1$ for the $x$-direction is studied exemplarily. The slope $m_{X 1}$ on rising segments and the maximal voltage difference $\Delta U_{X 1}$ are determined by the image parameters, i.e. the pixel size $\Delta x$, pixel dwell time $\Delta t$ and total number of pixels $N_{x}$ as well as a unique calibration parameter $\gamma$ for the specific setup, determined by imaging a reflective calibration grid with a known spacing. The slope of the applied voltage as well as the total voltage difference are given by

$$
\begin{gathered}
m_{X 1}=\overbrace{\frac{13.5 \mathrm{~V}}{85 \mu \mathrm{m}}}^{\gamma} \cdot \frac{\Delta x}{\Delta t}, \\
\Delta U_{X 1}=\underbrace{\frac{13.5 \mathrm{~V}}{85 \mu \mathrm{m}}}_{\gamma} \cdot \Delta x \cdot N_{x} .
\end{gathered}
$$

For measuring the scanner's response, the voltage applied to the scanner driver as well as the monitoring voltage on the scanner driver's output yielding the actual scan position are measured while acquiring an image of size $3 \mu \mathrm{m} \times 3 \mu \mathrm{m}$ with a pixel size of $20 \mathrm{~nm} \times 20 \mathrm{~nm}$ and pixel dwell time of $10 \mu \mathrm{s}$. The results are displayed in figure 3.12(a), featuring the input and

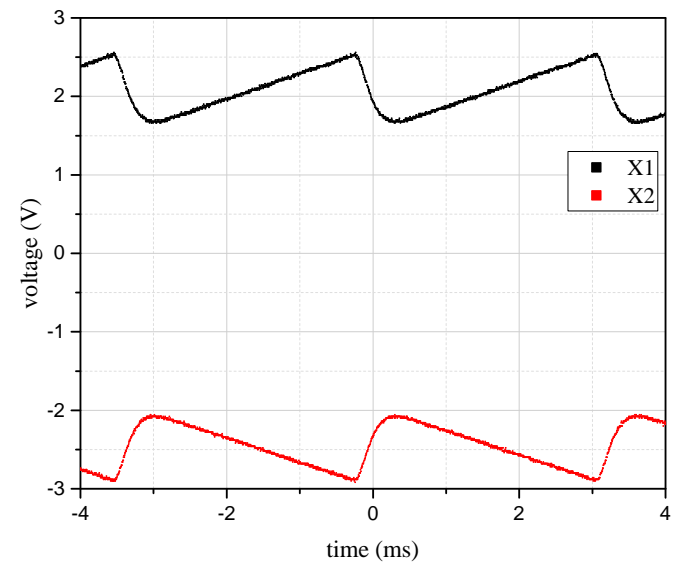

(a) Voltage on inputs X1 and X2.

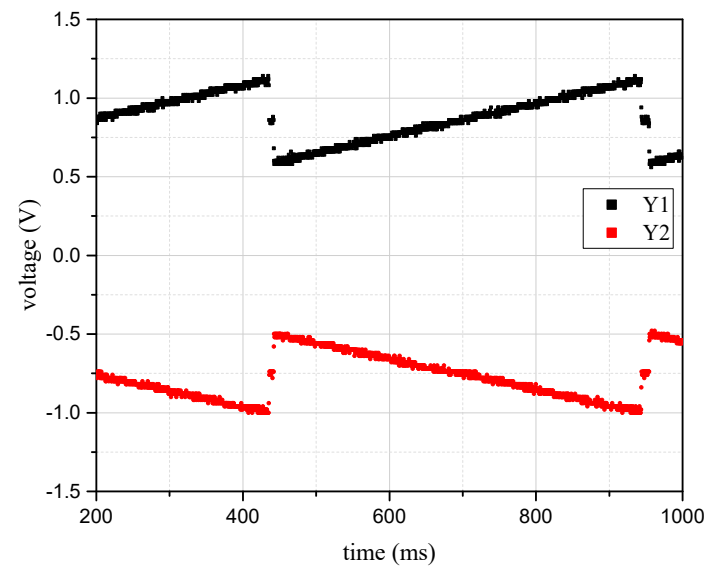

(b) Voltage on inputs Y1 and Y2.

Figure 3.11: Voltage applied by Imspector to the driver of the QUAD scanner on the four inputs for a pixel size of $20 \mathrm{~nm} \times 20 \mathrm{~nm}$, a pixel dwell time of $10 \mu$ s and an image size of $3 \mu \mathrm{m} \times 3 \mu \mathrm{m}$. Each pair of voltages is linearly dependent. Note the different time scales for the fast scan axis $\mathrm{X}$ and the slow scan axis $\mathrm{Y}$. 
output voltages as well as their difference. Due to the finite response time of the scanner, the monitored output voltage of the scanner driver has a temporal offset compared to its input, resulting in a voltage difference at fixed time points. This voltage difference, as displayed in figure 3.12 (a), can be translated into a positioning error, giving the maximal deviation between nominal and actual scan position. The deviation is dependent on the chosen pixel dwell time, as shown in figure 3.12(b). The functional dependence can be explained by solving the differential equations for describing the motion of a galvanometer (cf. appendix A). For a constant slope of the input voltage, as it is the case during a line acquisition, the nominal and actual scan position have, in the limit of large times, a constant offset $T$ in time, assuming the system to be critically damped. This offset $T$ only depends on the characteristics of the galvanometer, but not on the acquisition parameters. Translating the actual scan position back into a voltage, as done for the monitoring output, yields a constant delay of the output voltage compared to the input voltage. The resulting voltage difference amounts to

$$
\Delta U=m \cdot T=\frac{13.5 \mathrm{~V}}{85 \mu \mathrm{m}} \cdot \frac{\Delta x}{\Delta t} \cdot T
$$

with the slope $m$ as determined previously. Calculating the spatial deviation resulting from this voltage offset yields

$$
\Delta d=\frac{\Delta U}{13.5 \mathrm{~V}} \cdot 85 \mu \mathrm{m}=T \cdot \frac{\Delta x}{\Delta t} .
$$

These considerations are supported by the hyperbolic fit shown in figure 3.12(b) alongside with the measured data.

(a)

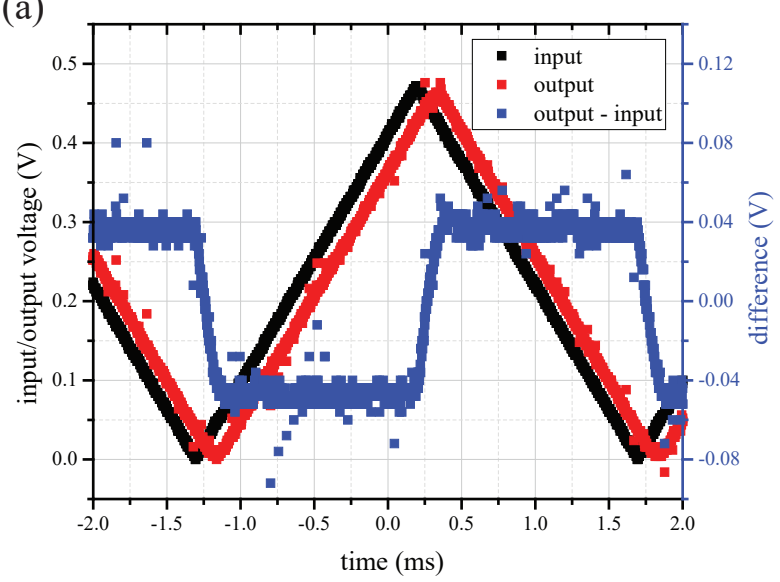

(b)

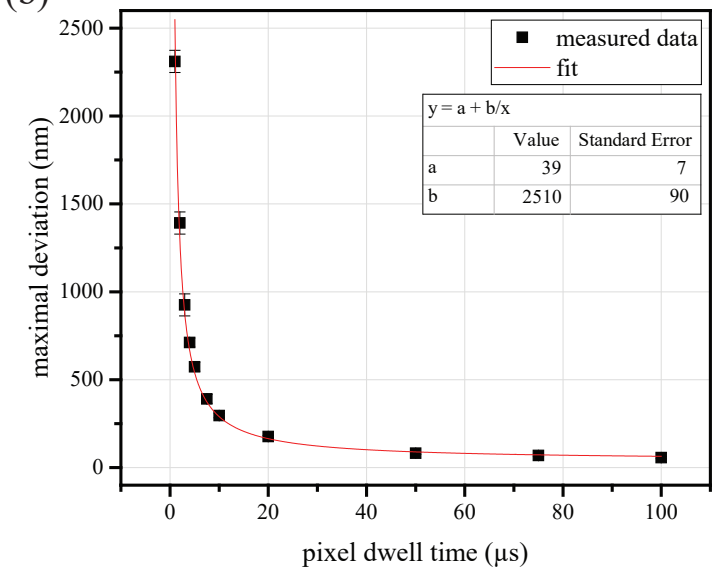

Figure 3.12: Measurement of the scanner's response as a function of the pixel dwell time. An image size of $3 \mu \mathrm{m} \times 3 \mu \mathrm{m}$ and a pixel size of $20 \mathrm{~nm} \times 20 \mathrm{~nm}$ are chosen as acquisition parameters. (a) Voltage applied to the scanner driver (input) and monitoring voltage on the scanner driver's output (left axis) as well as their difference (right axis) for a pixel dwell time of $10 \mu \mathrm{s}$. (b) Maximum spatial displacement between input and monitoring output as a function of the pixel dwell time. 
As seen from figure 3.12(b), a spatial displacement of more than $2 \mu \mathrm{m}$ between nominal and monitored scan position occurs for a pixel dwell time of $1 \mu \mathrm{s}$. Hence, for scanning techniques with different scan patterns, varying pixel dwell time or tracing possibilities there is the need for a scanning device with a faster response time, resulting in a lower displacement.

An overview of beam scanning techniques can be found in [MS04, featuring galvanometer scanners as well as acousto-optic and electro-optic scanners alongside less common scanning techniques. Most of them, like galvanometer scanners, do not meet the necessary requirements for speed and response time. Acousto-optic scanners are sufficiently fast, but the deflection is wavelength dependent and therefore not suitable for the presented experimental setup.

EODs as additional scanning devices fulfill all requirements presented beforehand, lacking only on the scan range which is, depending on the optical components thereafter, in the range of a few $\mu \mathrm{m}$. Since they are driven by an analog voltage, yet have no mechanical parts, they can deflect the beam almost instantaneously. The deflection is a function of the index dispersion and is relatively constant over the wavelength range of operation. To realize a larger scan range, they are combined with the galvanometer scanner utilized so far, a similar architecture as described in [RB14]. The specific properties of the EODs employed herein for such a combination is discussed in the following.

\subsubsection{Electro-optic deflectors}

As described in subsection 2.4.4. EODs deflect the beam by means of the electro-optic effect, resulting in a deflection angle after the EOD which depends on the applied voltage as well as the system's parameters. During beam scanning however, the angle of incidence of the beam at the objective's back focal plane is the measure of interest and needs to be constantly changed during the scanning process. Projecting the EOD's pivot point on the objective's back focal plane, i.e. aligning all beam scanning devices in conjugated planes, ensures that the beam remains in the center of the back focal plane during the entire scanning. The tilt results in a focus displacement of

$$
d=f_{\mathrm{obj}} \tan \alpha,
$$

defining $\alpha$ as the angle between optical axis and tilted beam and $f_{\text {obj }}$ as the objective's focal length. The total scan range of an EOD thus depends on the maximum deflection angle $\theta_{\text {def }}$ as well as on the magnification of the imaging system which conjugates the Pivot plane with the back-focal plane. For a magnification $M$, the deflection angle at the objective is given by

$$
\alpha=\frac{\theta_{\mathrm{def}}}{M},
$$




\begin{tabular}{|c|c|c|c|}
\hline \multicolumn{2}{|l|}{ Conoptics M311-A } & & \\
\hline $\begin{array}{l}\text { crystal material } \\
\text { aperture diameter } a \\
\text { deflection factor }\end{array}$ & $\begin{array}{l}\mathrm{AD}^{*} \mathrm{P} \\
2.5 \mathrm{~mm} \\
7.1 \mu \mathrm{rad} / \mathrm{V}\end{array}$ & $\begin{array}{l}n_{0} \\
\text { length } L \\
\text { voltage range }\end{array}$ & $\begin{array}{l}1.54 \\
200 \mathrm{~mm} \\
\pm 700 \mathrm{~V}\end{array}$ \\
\hline
\end{tabular}

Table 3.1: Properties of the electro-optic scan system.

yielding smaller scan ranges for higher magnification. Since over-illumination of the objective's back aperture is essential for a good image quality, the beam diameter $w_{\text {obj }}$ at the objective's back focal plane cannot be compromised on. Thus, the magnification is mainly determined by the beam width at the deflector, which has an upper limit as described by equation (2.37). The resulting maximal deflection angle is

$$
\alpha_{\max }=\frac{\theta_{\text {def, max }}}{w_{\text {obj }}}\left(a-\frac{1}{n_{0}} \theta_{\text {def, } \max } \cdot L\right) .
$$

In this work, two EODs are employed, of which one is utilized for $x$ - and one for $y$-scanning, implying that their deflection axes are rotated by $90^{\circ}$ with respect to each other. Since the electro-optic effect is only exhibited for one polarization direction of the incident beam, the polarization needs to be rotated accordingly, as realized by a half-wave plate in-between as shown in figure 3.1. A customized EOD model in combination with a standard voltage driver (Trek Inc., now part of Advanced Energy, USA) is chosen, as also described in Göt15. The relevant properties are summarized in table 3.1. The maximum possible beam diameter amounts to $1.85 \mathrm{~mm}$. For a total magnification of $M=7.3$ and an objective lens of focal distance $f_{\text {obj }}=1.8 \mathrm{~mm}$, a total scan range of $\pm 1.2 \mu \mathrm{m}$ is expected. This is experimentally verified by imaging fluorescent beads, utilizing the 1D STED depletion patterns as described in section 3.2. Here, the galvanometer scanner is employed for the scanning. Images for different voltages applied to the EODs are recorded and the induced deviation can be determined by measuring the relative position of single beads with respect to their position for the undeflected zero. For each EOD, the orientation of the 1D STED pattern is chosen such that the high-resolution axis and the deflection axis are parallel in order to improve location accuracy. The results are depicted in figure 3.13 for both deflectors and show the expected linear dependence on the voltage. At the edge of the voltage range, deviations with respect to the linear fits are visible, indicating that the maximum deflection of the EOD is reached. This maximum deflection is in good agreement with the expected total range of $\pm 1.2 \mu \mathrm{m}$.

For the experimental implementation of EODs, it is worth noting that the emitted fluorescence signal cannot be completely de-scanned for confocal detection due to the polarizationdependence of the electro-optic effect. Hence, a Glan-Thompson-prism in the beam path behind the EODs (cf. figure 3.1) filters the fluorescence signal accordingly. Assuming an isotropic fluo- 


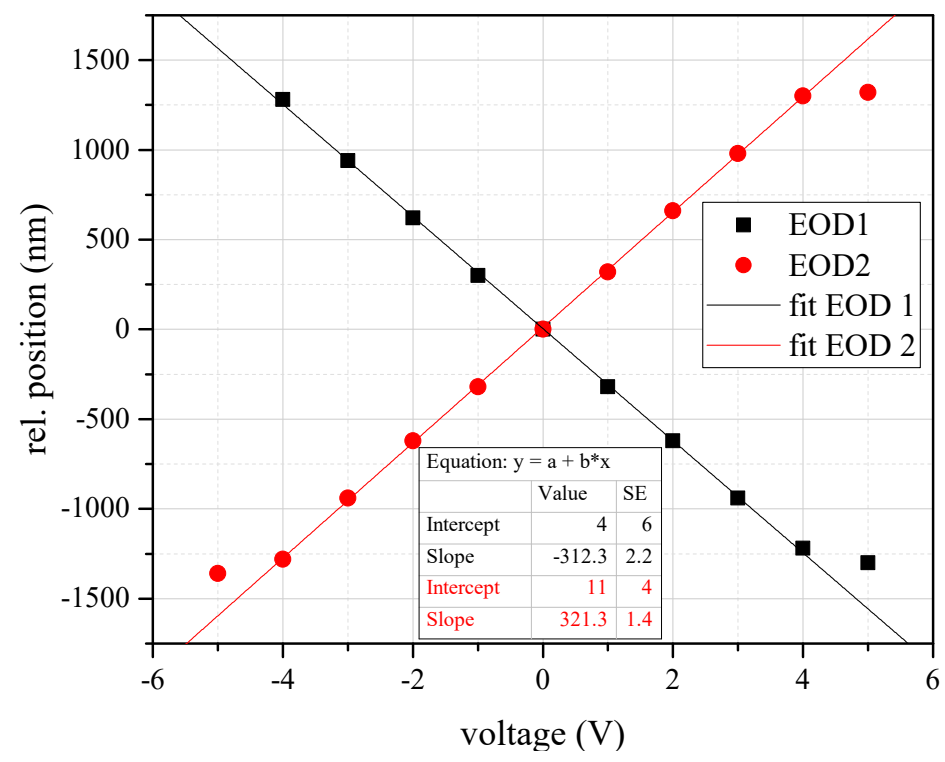

Figure 3.13: Focus shift on the sample due to EOD deflection as a function of the applied control voltage to the voltage amplifier. STED images with 1D depletion pattern of Crimson 40 fluorescent beads are acquired with the high-resolution axis aligned with the scan direction. A resolution enhancement of $k=5$ for a pixel size of $20 \mathrm{~nm}$ is chosen.

rescence emission due to a random distribution of fluorophores' dipole orientations, this implies a loss of half of the fluorescence signal.

\subsubsection{Assembly of the combined scan system}

As discussed beforehand, EODs fulfill all requirements regarding speed and deflection efficiency, but lack the scan range. In order to resolve this problem, two EODs - one for each scan axis are combined with a galvanometer scanner as outlined in the following.

Figure 3.14 shows a systematic sketch of the control of the combined scan system. Due to the limited number of analog outputs on the data acquisition card (see subsection 3.5.4), there are only two analog outputs for the scan system available, leaving one per scan direction. Hence, a home-built printed circuit board with adequate components, highlighted in red in figure 3.14 . is utilized to read and to output all necessary voltages. For a control of the galvanometer scanner, the voltages $U_{X 1}$ and $U_{Y 1}$ are read from the data acquisition card PCIe-7852 and the linearly related voltages $U_{X 2}$ and $U_{Y 2}$ are set. The EODs are used to compensate a mismatch between intended and actual scan position, as outlined in subsection 3.5.1 and induced by the slow reaction time of the galvanometer motors driving the mirrors of the QUAD scanner. The actual position can be accessed via the the monitoring output $U_{X 1 \text {,moni }}, U_{Y 1 \text {,moni }}$ of the scanner driver and is, for the two mirrors $X 1$ and $Y 1$, used as an input to the controller. The differences between $U_{X 1}$ and $U_{X 1 \text {,moni }}$ and between $U_{Y 1}$ and $U_{Y 1 \text {,moni }}$ are, appropriately scaled, rerouted to the voltage amplifiers for the EODs. In this way, the EODs compensate for the spatial displacement introduced by the galvanometer scanner. 


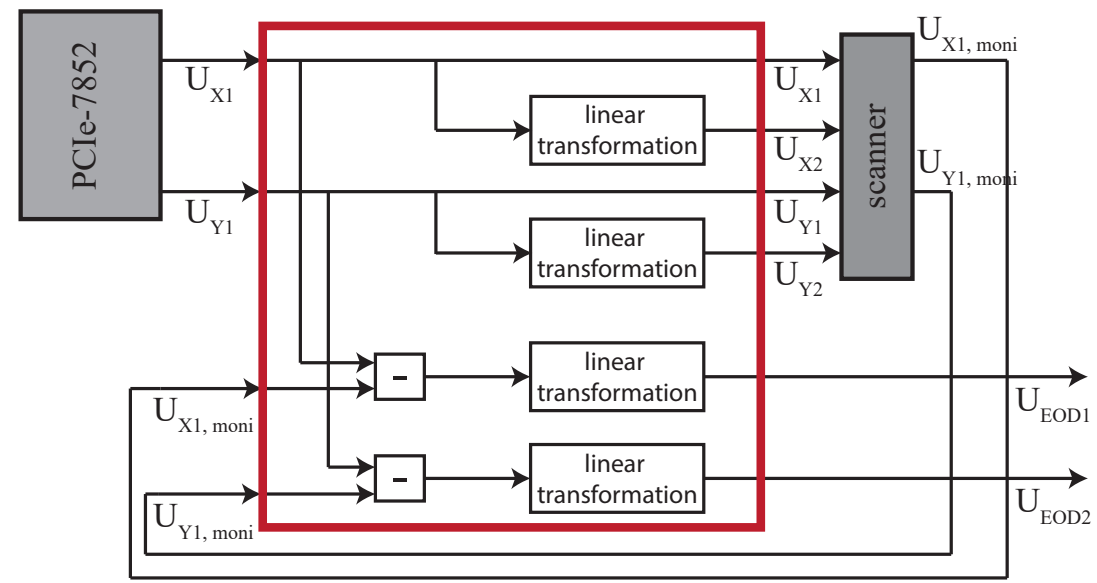

Figure 3.14: Sketch of the combined scan system's control. Two voltages for the scanner are output by the FPGA card (PCIe-7852). They serve as inputs to the printed circuit board (red) and are subsequently linearly transformed. The monitoring output voltages of the scanner are used as additional inputs to derive the voltages for the EOD drivers.

\subsubsection{LabVIEW program}

As mentioned beforehand, the Imspector software utilized for standard image acquisition does not allow the necessary flexibility for adaptive scanning techniques as described in this section. A modification of the scan pattern is not accessible once the imaging parameters are chosen, and moreover a direct access to the acquired signal is not available. This poses the necessity for a different acquisition software. The multifunction reconfigurable I/O device (PCIe-7852) used for controlling all analog and digital in- and outputs has a direct support for LabVIEW as development environment which is therefore used as programming interface. This subsection is dedicated to outlining the most important features of the LabVIEW program associated with the setup's operation.

The LabVIEW program consists of two parts: a host program running on the host computer and an FPGA program, which is compiled on the FPGA of the PCIe-7852 card. The communication between these programs is managed via FIFO (First In First Out) arrays, which has the advantage of unsynchronized write- and read-processes. The host program reads the acquisition parameters like pixel dwell time and size, field of view, number of pattern orientations or RESCue parameters from the user interface. It subsequently calculates all dependent variables like the necessary voltages for the scan system's driver as well as for the Pockels cells' drivers and hands those over to the FPGA program. It starts the acquisition and can also stop the acquisition via a hand-over STOP parameter to the FPGA program. Furthermore, it provides functionalities for saving and displaying the acquired data read from the FPGA program.

The FPGA program runs time-independently of the host program. Compared to a standard computer program running on the computer's CPU, which is a sequential processing device, a 


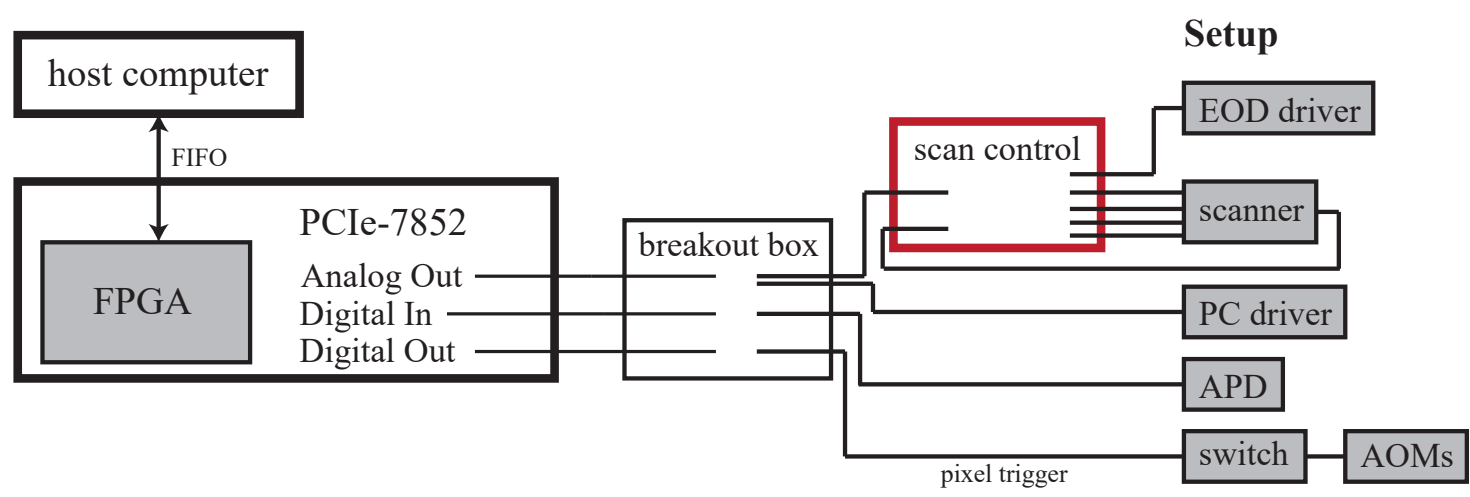

Figure 3.15: Overview of the setup's operation. A LabVIEW routine running on the host computer reads the variables like pixel dwell time, pixel size, field of view and RESCue parameters. It calculates all dependent values and submits them via a FIFO array to another LabVIEW program running on the FPGA. This program, once compiled, is running independently of the host computer's CPU and operating system. It controls analog outputs as well as digital in- and outputs, which are via a breakout box physically connected to all relevant devices of the experimental setup. Note that only one (out of two) EOD and only one (out of six) Pockels cell's driver is shown for simplification.

program compiled on an FPGA can fully exploit the FPGA's parallel processing capabilities. This includes the use of Single Cycle Timed Loops (SCTL), for which the entire algorithm of the loop is executed in a single tick of a $200 \mathrm{MHz}$-clock. This provides accurate operational timing, necessary to correctly determine the pixel dwell time.

The FPGA program controls the scan pattern by writing the voltage values $U_{X 1}$ and $U_{Y 1}$ for the nominal scan positions to analog outputs. Similarly, the pattern orientation is controlled via the voltages applied to the six Pockels cells, which are also managed by the FPGA program on the remaining analog outputs. Since there are in total only eight analog outputs available, the remaining scan control is outsourced as described in the previous subsection. The APD signal is read via a digital input and directly analyzed for the optimization of scan direction and pixel dwell time. For the laser control, a pixel trigger is generated on one of the digital output lines and, post-processed by an electronic switch, routed to the AOMs.

An overview of the setup's control is depicted in figure 3.15. It features the host computer and the FPGA, where the according LabVIEW programs are running on, as well as the setup's components controlled via the program. A more detailed description of the LabVIEW routines depending on the concrete application is given in the following.

FastRESCue The workflow of the LabVIEW program for FastRESCue is graphically outlined in figure 3.16. The innermost part is an SCTL, cycling on each pixel while the signal from the APD is acquired. It stops if either one of the two RESCue conditions is met, i.e. the maximum number of counts is reached or the minimum number of counts has not been collected in the specified time range, or if the pre-defined pixel dwell time is reached. The col- 


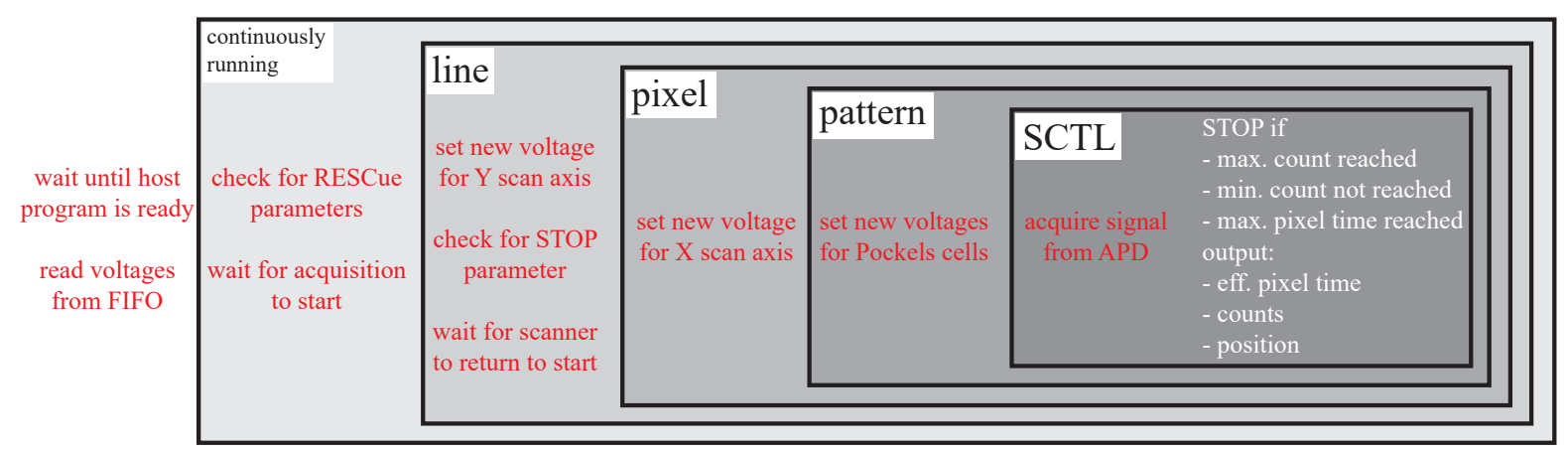

Figure 3.16: Sketch of the workflow of the FastRESCue LabVIEW FPGA program. One continuously executing while loop contains the for loops for the frame, line and pixel acquisition. At the beginning of each loop, the corresponding voltages for the scan system or Pockels cells are set. Before each line acquisition, the STOP parameter is checked and the acquisition continued or interrupted accordingly. A waiting time at the start of each line additionally guarantees that the galvanometer motors for the $\mathrm{X}$ scan mirrors can return to their starting positions. On each pixel, a SCTL runs until one of the stop conditions is met. The acquired signal count from the APD as well as the effective pixel dwell time are output to the host program.

lected signal count as well as the effective pixel dwell time and the actual position are output to the host program via a FIFO array. The surrounding for loops iterate over the number of pattern orientations on each pixel, the pixels per line and the lines per frame. At the beginning of each loop execution, the voltages for Pockels cells, $\mathrm{X}$ scan axis and $\mathrm{Y}$ scan axis are set respectively. Additionally, before each line acquisition, the STOP parameter from the host program is checked and the acquisition continued or interrupted accordingly. A waiting time moreover ensures that the $\mathrm{X}$ scan mirrors are reset to their starting positions at the start of the line. An overall while loop, which is independently running no matter whether the program is acquiring or not, is continuously checking for the start of the acquisition and updating the RESCue parameters. All other parameters, mainly the voltages for scanning and pattern generation, are read from the FIFO array before the start of this loop.

Filament tracing For the tracing of a filamentous structure, an adaptable scan pattern is of crucial importance, as outlined in the following. The workflow of the self-written LabVIEW program for this filament tracing is depicted in figure 3.17 .

The basic concept of the algorithm is the scanning of blocks of a certain number of either vertical or horizontal lines on a pre-defined grid of pixels. The lines have a fixed length, which is chosen such that the confocal width of the filament is fully covered. On each scanned pixel, the voltages for the scan system are set appropriately and a for loop iterates over the number of pattern orientations. For each orientation, the voltages for the Pockels cells are output, before a SCTL is employed to register the signal count from the APD. The resulting detector signal is output to the host program for image visualization and post-processing and is additionally considered as a criterion to define the further scanning path: At the end of each block, i.e. after a certain number of lines have been acquired, the positioning of the next block is determined 


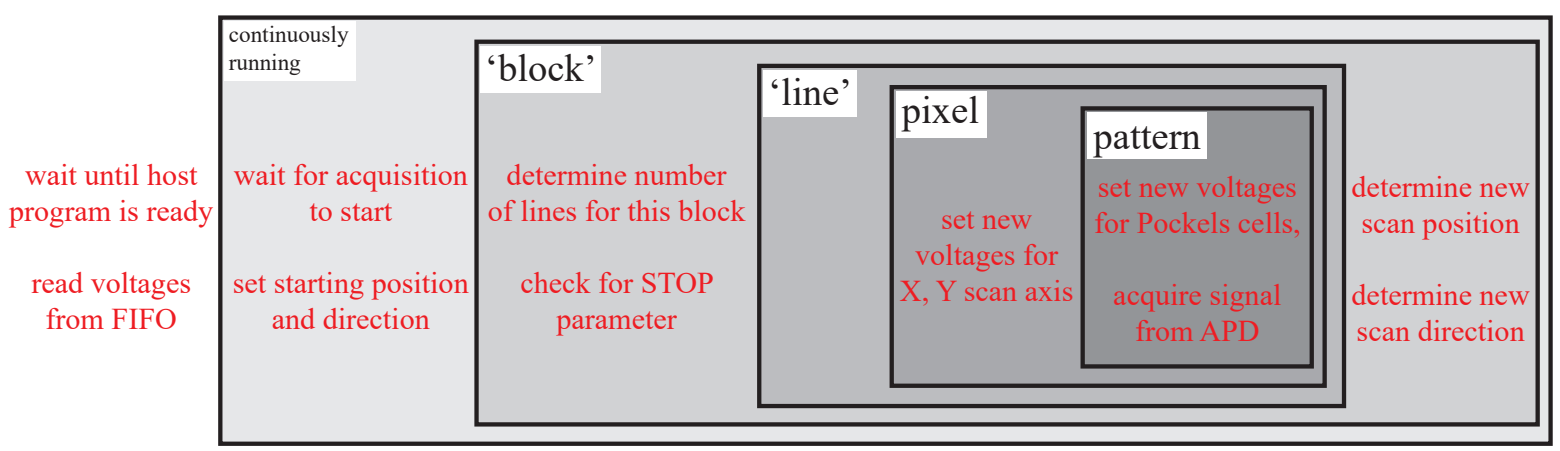

Figure 3.17: Sketch of the workflow of the tracing LabVIEW FPGA program. One continuously executing while loop contains for loops for iterating over various pixel positions and pattern directions, which are determined by a decision algorithm (see details in section 4.3. On each pixel, a SCTL runs to acquire signal from the APD, which is subsequently output to the host program. At the beginning of each loop, the corresponding voltages for the scan system or Pockels cells are set. Before each step, the STOP parameter is checked and the acquisition continued or interrupted accordingly.

based on the registered counts in the last line of the previous block. Here, both the actual position of the filament as well as its current direction are taken into consideration as described in detail in section 4.3. In this way, the scan position for the next block as well as the orientation of the lines for the scan (either vertical or horizontal, cf. section 4.3) are determined, with the number of lines being calculated at the beginning of the next iteration (for loop 'block' in figure 3.17). A STOP parameter allows the user to interrupt the acquisition at the beginning of each block. The for loop 'block' (cf. figure 3.17) iterates over a pre-defined number of blocks and thus indirectly determines the length over which a filament is scanned. This for loop is embedded in an overall while loop, which runs independently of the acquisition and allows to set the starting position and starting direction for the filament tracing. The scan voltages for the underlying pixel grid as well as the voltages for the Pockels cells are read from a FIFO, alongside with all other acquisition parameters, before the start of the outermost while loop. 



\section{Results and Discussion}

The unique features of the beforehand presented experimental setup allow to explore novel ways for photon-efficient and adaptive STED imaging modes. In the following, three different approaches are presented. First, tomoSTED microscopy is enhanced by employing conical diffraction for generating the STED depletion pattern. In this way, a pixel-wise switching of the pattern orientation can be realized and is first demonstrated on fluorescent microspheres (see subsection 4.1.1) and second on biological samples (see subsection 4.1.2).

Subsequently, FastRESCue, a novel variant of RESCue aiming on reducing both light dose and acquisition time, is presented. It makes use of the EOD-assisted fast scanning capability of the setup by employing adaptive illumination schemes. A characterization of the scan system's performance (see subsection 4.2.1) is prepended to results on both fluorescent microspheres as well as several biological samples (see subsection 4.2.2).

The last section of this chapter (section 4.3 ) is dedicated to a novel approach for structure tracing, enabled by the unique combination of fast pattern generation and fast scanning. By only scanning a single filament instead of the whole field of view, the acquisition speed can be improved even further.

\subsection{TomoSTED microscopy with pixel-wise switching}

Conical diffraction has been identified in section 3.2 as a suitable candidate for generating a 1D depletion pattern. A comparison of intensity profiles of simulated patterns suggests a similar resolution enhancement for both a pattern generated by conical diffraction and a pattern generated by a conventional phase mask (see figure 3.3. Pockels cells allow a fast rotation of the pattern orientation, as discussed in section 3.3, and thus enable switching on a pixel level. In the following, the depletion pattern is fully characterized employing measurements on fluorescent microspheres. Please note that all measurements in this section are conducted with pulse gating in order to suppress undesired confocal contributions in the STED images.

\subsubsection{Imaging of fluorescent microspheres}

As described in section 2.2 the applied STED laser power is easily accessible experimentally. However, it is not the only relevant parameter which governs the fluorescence depletion and thus the achievable resolution enhancement. The influences of e.g. the fluorophore properties (STED cross-section), the laser parameters (repetition rate, wavelength) and the microscope (NA) can be merged into a single parameter, the saturation power $P_{\text {sat }}$. It is given by the power necessary to deplete the fluorescent signal to half, can be determined experimentally and allows to compare the performance of different STED patterns regardless of the utilized microscope.

Therefore, a STED focus with a Gaussian intensity profile is utilized to deplete fluorescence 
and determine $P_{\text {sat }}$. The measurements are conducted for fluorescent microspheres at varying STED laser power (for all sample protocols refer to appendix B). In order to compensate for bleaching artifacts, a confocal scan is performed before and after each STED acquisition and the average of both scans is taken for the further analysis. The sum of the signal count over the area of $700 \mathrm{~nm} \times 700 \mathrm{~nm}$ is determined for both confocal scans and the STED acquisition. Subsequently, the respective quantity for the STED image is divided by the average signal count for the confocal images, yielding the ratio of the signal counts at each STED laser power. The results are averaged over several beads and displayed in figure 4.1. An exponential fit is used to approximate the data and allows to determine the saturation power $P_{\text {sat }}=(0.497 \pm 0.018) \mathrm{mW}$ from the fit parameters.

The suitability of the suggested STED depletion pattern generated by conical diffraction is experimentally verified. Both the depletion pattern generated by conical diffraction for $\rho_{0} \approx 1.3$ and a pattern generated conventionally by a $0 / \pi$ phase plate (data taken from [Krü17]) are employed for imaging fluorescent microspheres for different STED laser powers. Exemplary measurements with a depletion pattern generated by conical diffraction for two pattern orientations are depicted in figure 4.2(a) and (b): The upper left corner of each image shows a confocal image, the lower right corner a STED image with one-dimensional resolution enhance-

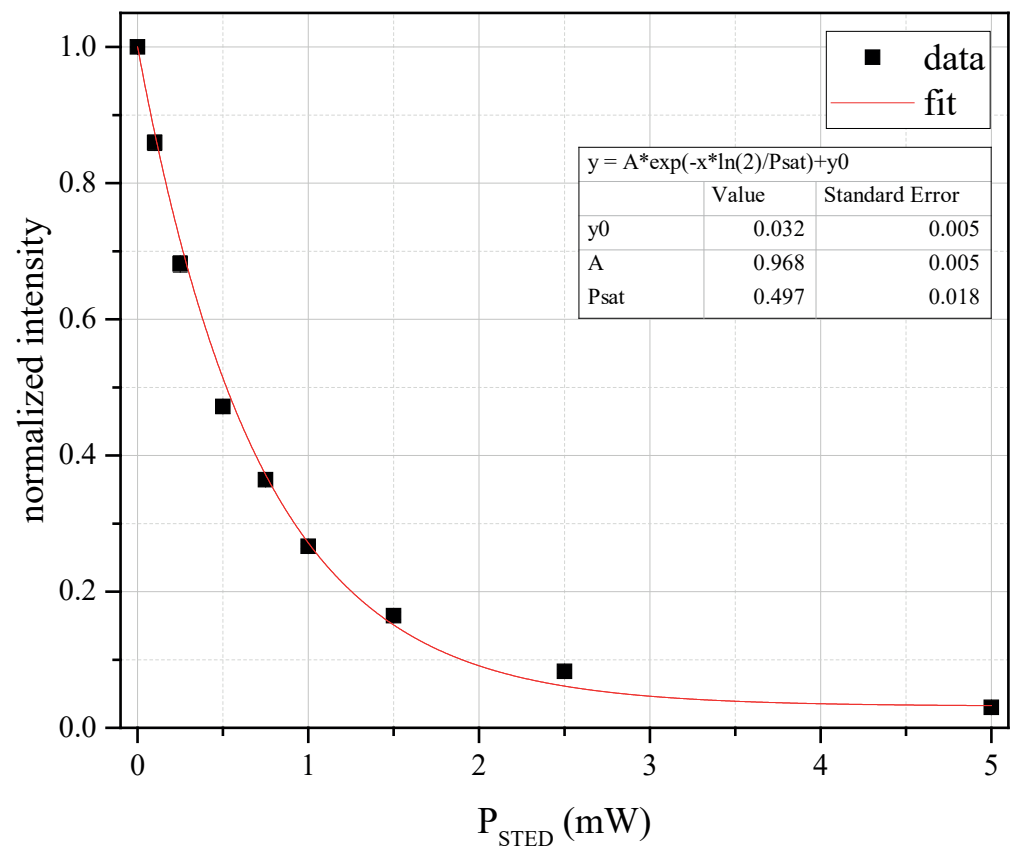

Figure 4.1: Utilizing a Gaussian shaped STED intensity distribution and circular polarization, images of Crimson 40 microspheres are recorded for different STED laser powers. The resulting signal count as sum over the area of $700 \mathrm{~nm} \times 700 \mathrm{~nm}$ is compared to the respective quantity in confocal mode. To account for bleaching, the signal count for the confocal mode is determined as average of a confocal scan before and after the STED acquisition. The average of this ratio over 10 microspheres is shown here together with an exponential fit. The saturation power can be determined as $P_{\text {sat }}=(0.497 \pm 0.018) \mathrm{mW}$. 
ment. The corresponding STED PSFs measured on gold beads are depicted in the insets.

A quantitative analysis is conducted by measuring and averaging the FWHM in the direction perpendicular to the orientation of the 1D STED pattern, i.e. the high-resolution direction. The measurements are done for the two different pattern orientations shown in figure $4.2(\mathrm{a}, \mathrm{b})$ as well as for one orientation of the $0 / \pi$ phase plate (data taken from [Krü17], a homogeneous resolution increase for different orientations in this case is demonstrated therein) for varying STED laser power. The measured FWHM is corrected for the size of the microspheres (cf. [Krü17]) to obtain the effective resolution $\Delta_{\text {STED }}$ as a function of the saturation factor $\zeta$, determined by scaling the applied STED laser power with the saturation power. The data shown in figure 4.2(c) are fitted according to equation (2.11) by

$$
\Delta_{\mathrm{STED}}=\Delta_{\mathrm{conf}} \cdot \frac{1}{\sqrt{1+\frac{1}{8 \ln 2} \Delta_{\mathrm{conf}}^{2} \cdot a \cdot \zeta}}
$$
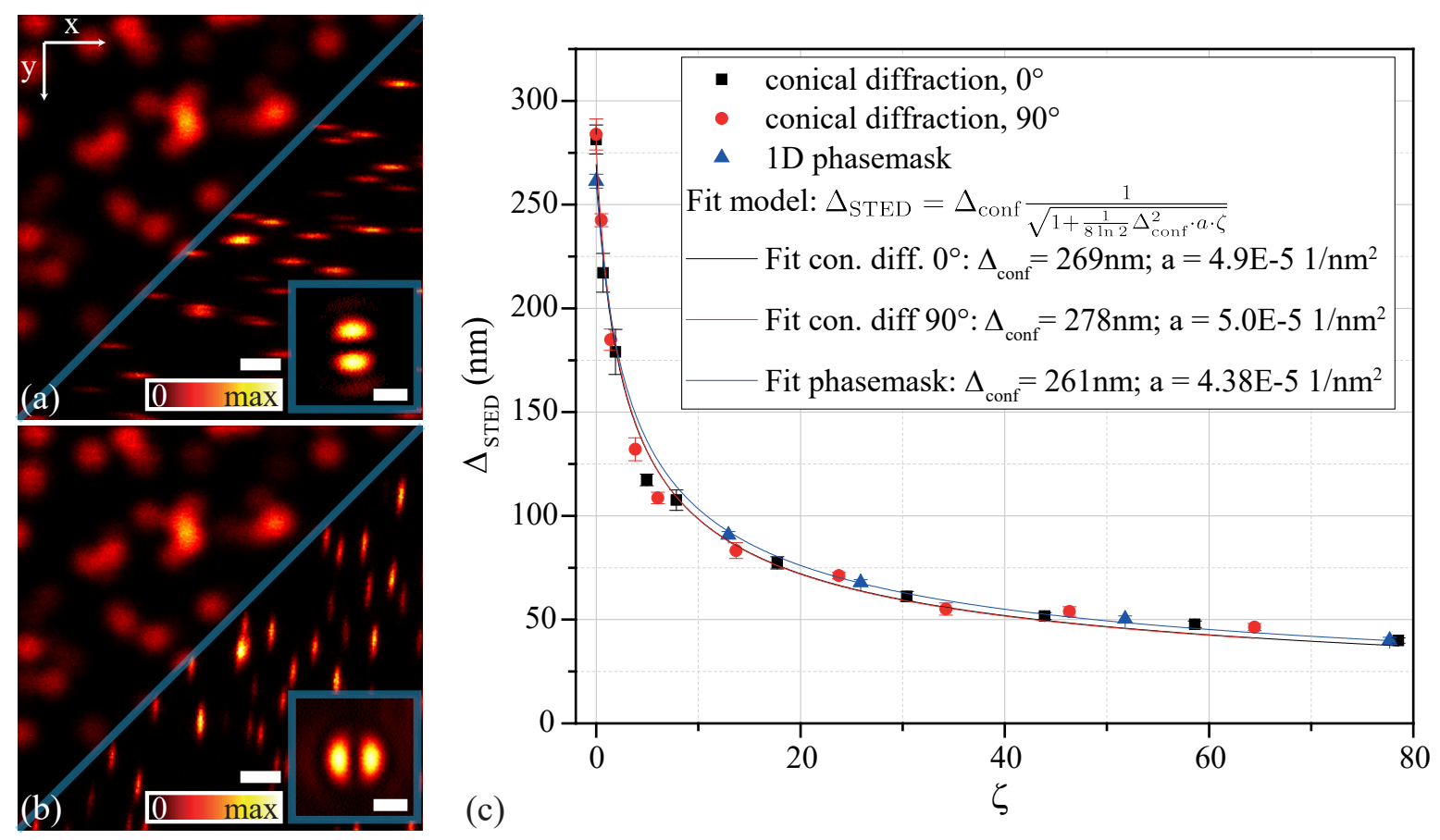

Figure 4.2: (a,b) Confocal (upper left corner) and STED (lower right corner) images of fluorescent microspheres (Crimson 40, $48 \mathrm{~nm}$ diameter) with one-dimensional resolution enhancement for a depletion pattern generated by conical diffraction enclosing an angle of $0^{\circ}$ (a) and $90^{\circ}$ (b) with the $x$-axis. The scale bar indicates a length of $2 \mu \mathrm{m}$. Here, $P_{\text {STED }}=38 \mathrm{~mW}, P_{\text {exc }}=3.2 \mu \mathrm{W}$, the pixel dwell time is set to $p T=30 \mu \mathrm{s}$ and the pixel size to $p x=20 \mathrm{~nm}$. The insets show the respective STED PSFs visualized by gold bead measurements (scale bar $0.5 \mu \mathrm{m}$ ). (c) Dependence of the resolution on the STED laser power for a conically diffracted intensity distribution with orientation of $0^{\circ}$ (black), a conically diffracted intensity distribution with orientation of $90^{\circ}$ (red) and an intensity distribution generated by a $0 / \pi$ phase mask (blue). The experimental data are well described by equation (4.1). The respective Levenberg-Marquardt fits have fitting parameters $\Delta_{\text {conf }}=(269 \pm 15) \mathrm{nm}, a=(4.9 \pm 0.4) 10^{-5} \mathrm{~nm}^{-2}$ (black curve), $\Delta_{\text {conf }}=(278 \pm 12) \mathrm{nm}, a=(5.0 \pm 0.5) 10^{-5} \mathrm{~nm}^{-2}$ (red curve), $\Delta_{\text {conf }}=(261 \pm 4) \mathrm{nm}$, $a=(4.38 \pm 0.12) 10^{-5} \mathrm{~nm}^{-2}$ (blue curve). 
with $\Delta_{\text {STED }}, \Delta_{\text {conf }}$ being the FWHM for the effective STED and the confocal PSFs, respectively and $a$ the depletion pattern steepness. The fitting parameters are given by $\Delta_{\text {conf }}=$ $(269 \pm 15) \mathrm{nm}, a=(4.9 \pm 0.4) 10^{-5} \mathrm{~nm}^{-2}$ for the horizontally oriented conical diffraction STED pattern, $\Delta_{\text {conf }}=(278 \pm 12) \mathrm{nm}, a=(5.0 \pm 0.5) 10^{-5} \mathrm{~nm}^{-2}$ for the vertically oriented conical diffraction STED pattern and $\Delta_{\text {conf }}=(261 \pm 4) \mathrm{nm}, a=(4.38 \pm 0.12) 10^{-5} \mathrm{~nm}^{-2}$ for a vertically oriented $0 / \pi$ phase plate. Hence, the parameter $a$ is similar for the two orientations of the depletion pattern generated by conical diffraction and even larger than for the reference measurement with the $0 / \pi$ phase plate. This is also expected from the simulation results presented in figure 3.3 .

Consequently, depletion patterns generated by conical diffraction are, in terms of resolution enhancement, comparable to or even slightly more efficient than the pattern generated by a $0 / \pi$ phase plate. Exploiting the high switching rates possible with Pockels cells, a rotation on
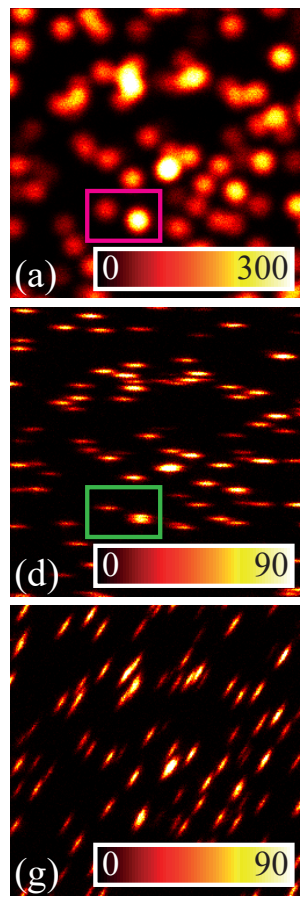
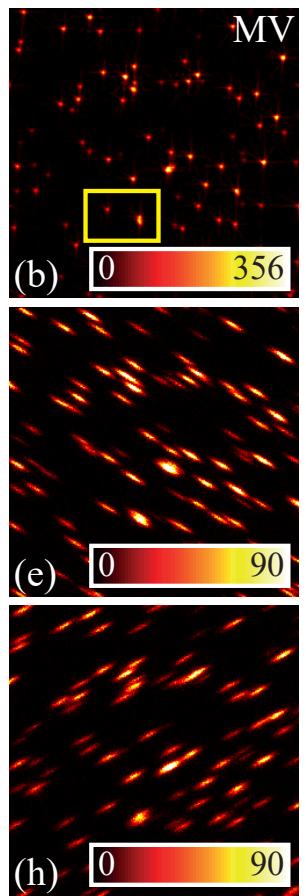
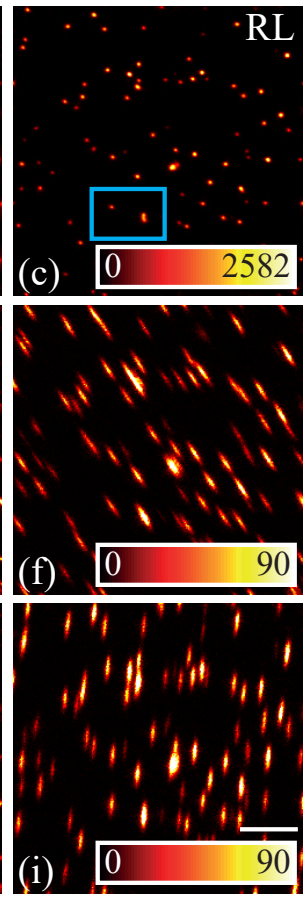
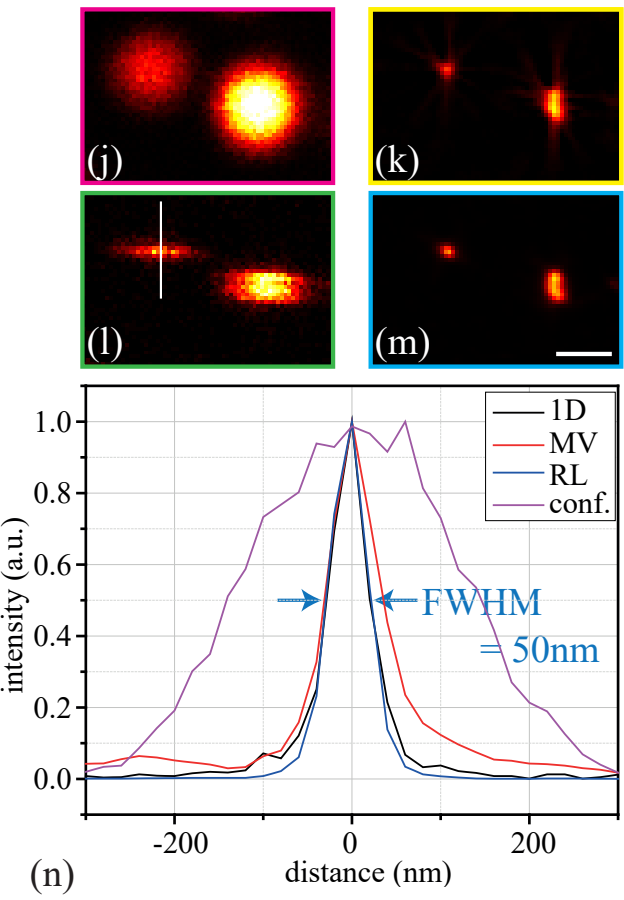

(n) Figure 4.3: TomoSTED microscopy on Crimson 40 microspheres: Comparison of maximum-value
reconstruction and Richardson-Lucy deconvolution. (a) Confocal scan of Crimson 40 microspheres at an excitation power of $P_{\text {exc }}=7.9 \mu \mathrm{W}$, a pixel size of $p x=20 \mathrm{~nm}$ and a pixel dwell time of $p T=90 \mu \mathrm{s}$. (b,c) Reconstructed images for the tomoSTED acquisition with 6 pattern orientations at a STED laser power of $P_{\mathrm{STED}}=46 \mathrm{~mW}$. The individual images for the six orientations are displayed in (d-i). The maximum-value reconstruction (b) has a significantly lower signal count than the Richardson-Lucy deconvolution (c), which takes full advantage of all available signal. The Richardson-Lucy deconvolution was conducted with 20 iteration steps and $\alpha_{R c h}=0.0001$. Closeups of (j) the confocal, (l) the sub-image with according pattern orientation, $(\mathrm{k})$ the maximum-value reconstruction and $(\mathrm{m})$ the Richardson-Lucy deconvolution are shown with the respective line profiles (n) averaged over 3 neighboring lines. The scale bar indicates a length of $1 \mu \mathrm{m}$ (a-i) and $200 \mathrm{~nm}$ in the closeups (j-m). 
a single-pixel level can thus be realized. Such a pixel-wise acquisition is demonstrated on fluorescent microspheres, with the results shown in figure 4.3. Sub-images for six different pattern orientations (d-i) are displayed alongside with the confocal image (a) and the reconstructed images (b,c). The maximum-value reconstruction (b) is performed as described in section 3.4 . It shows artifacts due to the insufficient sampling with pattern orientations (cf. figure 2.5) for the here applied resolution enhancement of $k \approx 6$, corresponding to an optimum number of 10 pattern orientations. The under-sampling yields a slightly larger FWHM in the image, as can be seen from line profiles drawn over a single bead. The corresponding profile is indicated in the closeups (j-m) and quantitatively shown in (n). The Richardson-Lucy deconvolution $(\mathrm{c}, \mathrm{m})$, performed as described in section 3.4 for 20 iteration steps and regularization parameter $\alpha_{R c h}=0.0001$, can compensate for the insufficient sampling, as shown in [Krü17. It yields the same image FWHM of $50 \mathrm{~nm}$ as in the raw data, corresponding to a FWHM of $42 \mathrm{~nm}$ of the effective PSF.

As a consequence of the results shown in figure 4.2, tomoSTED microscopy with the here presented depletion pattern yields the same advantages over 2D STED microscopy as for the variant presented in [Krü17]. Namely, the same resolution enhancement for each sub-image is obtainable at only half of the STED laser power compared to 2D STED microscopy. For an adequate comparison of the light dose employed on the sample, the pixel dwell time per sub-image for tomoSTED microscopy needs to be scaled such that the total pixel dwell time amounts to the respective quantity utilized for 2D STED microscopy. As shown in [Krü17], the necessary light dose for image acquisition can be reduced even further by imaging with $N_{\text {opt }} / 2$ number of pattern orientations, yielding an artifact-free reconstruction when employing the Richardson-Lucy deconvolution. In this way, imaging with only one fourth of the light dose compared to 2D STED microscopy is possible, as demonstrated in the following on cellular structures.

\subsubsection{Imaging of microtubules in fixed Vero cells}

The beforehand characterized depletion pattern is subsequently employed for imaging biological structures. Microtubules in Methanol-fixed Vero cells are stained with Abberior STAR 635P as fluorescent dye via antibody-staining. A confocal image is shown in figure 4.4(a) (lower right corner) with the scale bar indicating a length of $2 \mu \mathrm{m}$. For a tomoSTED image, a decision on the number of pattern orientations for the acquisition needs to be taken based on the resolution enhancement as described in section 2.2. For a chosen STED laser power of $38 \mathrm{~mW}$, a resolution enhancement of $k \approx 7$ is expected, necessitating at least six different pattern orientations for an artifact-free Richardson-Lucy reconstruction (see figure 2.5 and the description thereof). These six equally spaced pattern orientations are switched on a single-pixel level with a pixel dwell time of $5 \mu$ s per direction, amounting to a total pixel dwell time of $30 \mu \mathrm{s}$. By scaling the pixel dwell times of the sub-images, a comparable pixel dwell time as in the 2D case is ensured 
[Krü17, enabling imaging at half of the light dose compared to conventional 2D STED microscopy. Reordering the signal yields a sub-image per direction, as displayed in figure 4.4(c-h) on a subset of the whole field of view. A two-dimensionally super-resolved tomoSTED image (figure 4.4(a), upper left corner) is reconstructed from these sub-images by a Richardson-Lucy algorithm with 30 iteration steps and regularization parameter $\alpha_{R c h}=0.0001$. A zoom-in is shown in figure 4.4(b). The analysis of small fluorophore aggregations yields a width of less than $40 \mathrm{~nm}$, which confirms the expected 7 -fold resolution enhancement (data not shown). This resolution is sufficient to resolve immunolabeled microtubule filaments. Their expected diameter amounts to approximately $60 \mathrm{~nm}$, consisting of the size of the unlabeled microtubule of around $25 \mathrm{~nm}$ and the size of the antibodies WRO78. Indeed, an exemplary line profile perpendicular to a filament (figure 4.4(i)) shows a measured structure size of $58 \mathrm{~nm}$.

In order to further reduce the light dose, either less pattern orientations or a shorter pixel dwell time can be employed. The number of pattern orientations is critical for an artifactfree reconstruction and is already reduced to the minimum possible value for Richardson-Lucy deconvolution. On the other hand, the collected signal is still relatively high, allowing a re-

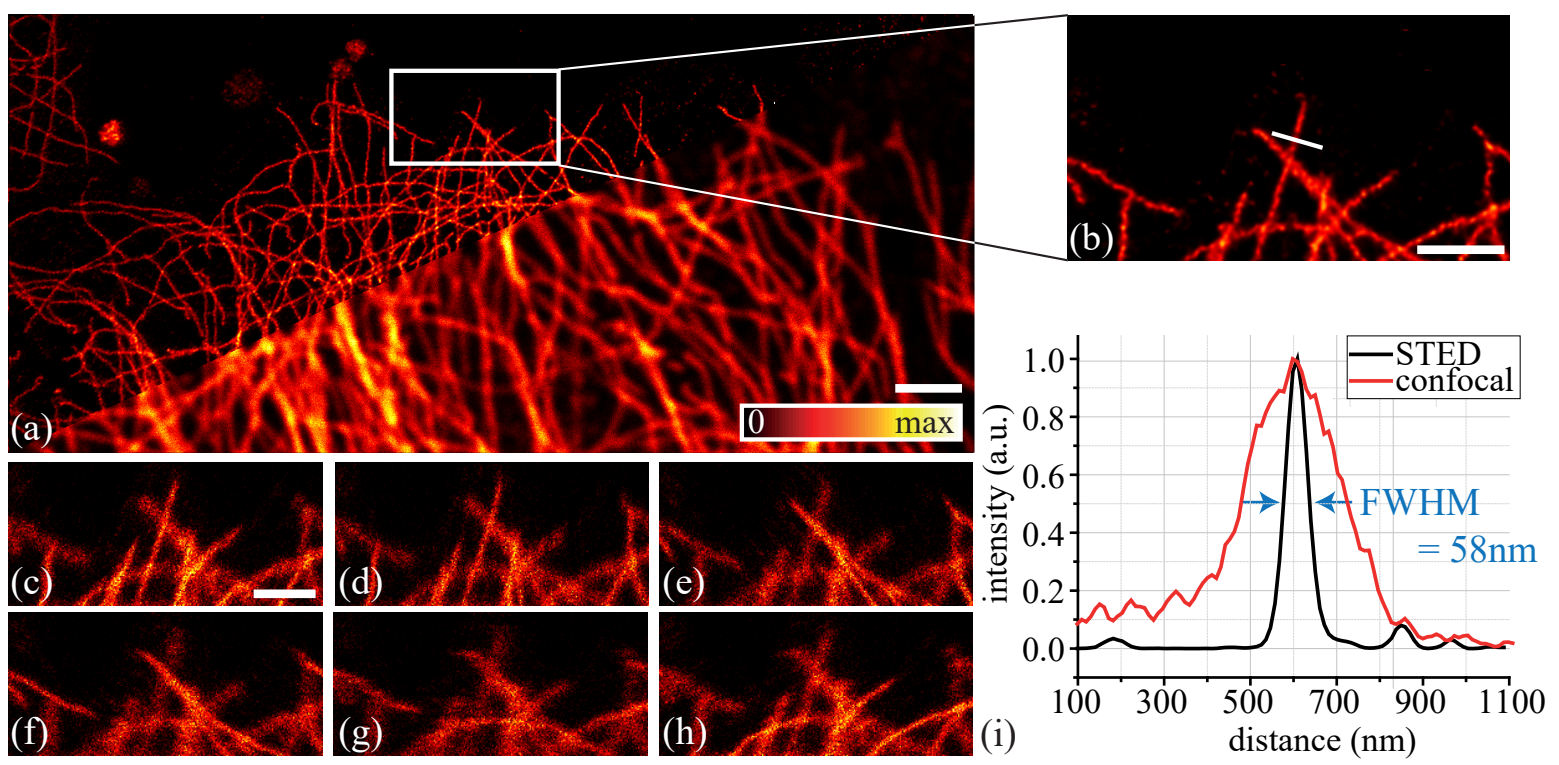

Figure 4.4: (a) Confocal image (lower right corner) and reconstructed two-dimensionally resolved STED image (upper left corner) of $\alpha$-tubulin in fixed Vero cells (fixed in MeOH, Abberior STAR 635P as fluorescent dye, embedded in Mowiol), based on measurements with six pixel-wise switched pattern orientations. The scale bar indicates a length of $2 \mu \mathrm{m}$. Here, $P_{\mathrm{STED}}=38 \mathrm{~mW}, P_{\mathrm{exc}}=3.2 \mu \mathrm{W}$, the pixel dwell-time is set to $p T=5 \mu \mathrm{s}$ per pattern orientation and the pixel size to $p x=20 \mathrm{~nm}$. The reconstruction was performed by employing the Richardson-Lucy deconvolution with 30 iteration steps and $\alpha_{R c h}=0.0001$. (b) shows a close-up of the reconstructed STED image, with (c-h) showing the six sub-images for the six pattern orientations for the same area (scale bar $1 \mu \mathrm{m}$ ). An exemplary line profile (averaged over 5 neighboring lines) of a filament for excitation and STED image as visualized in (b) is shown in (i), exhibiting a FWHM of $58 \mathrm{~nm}$ in the STED image. 

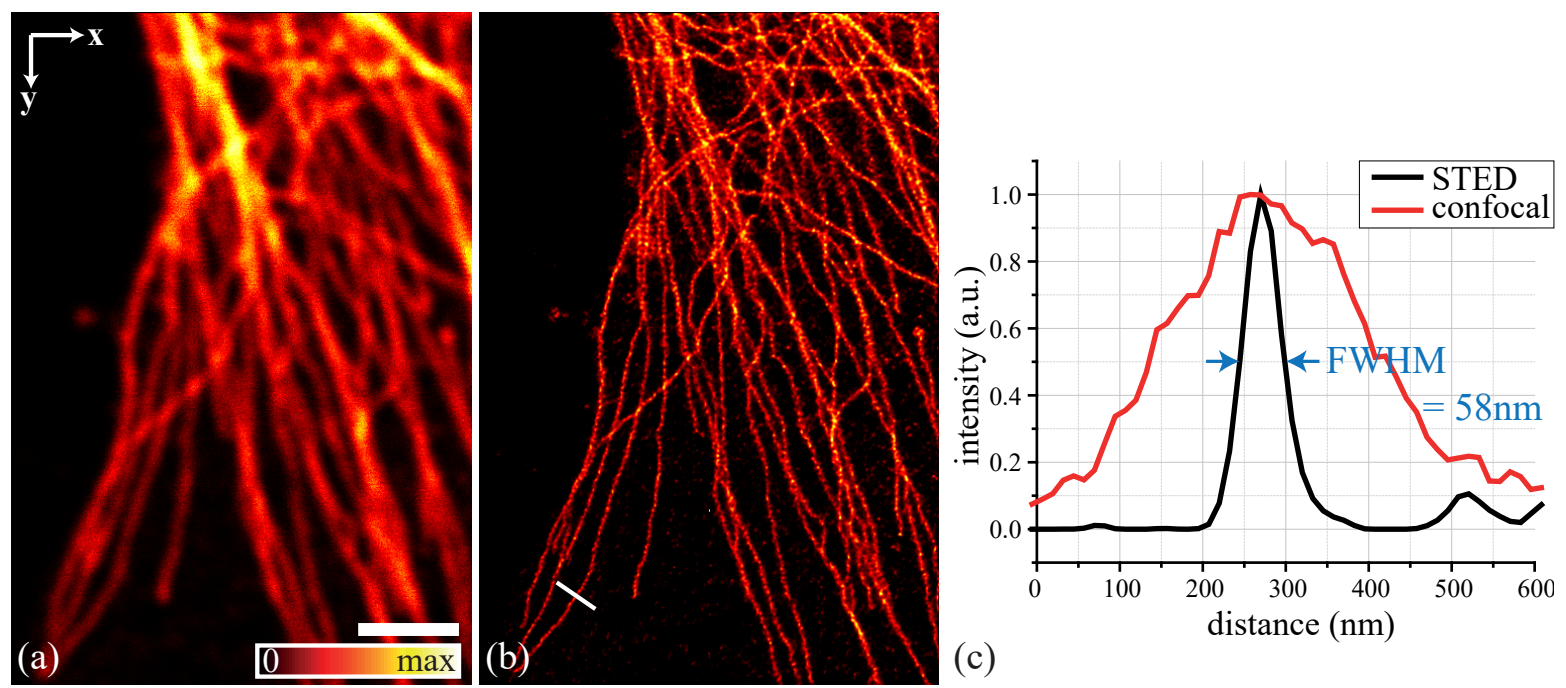

Figure 4.5: (a) Confocal and (b) reconstructed tomoSTED image (Richardson-Lucy deconvolution with 30 iterations, $\alpha_{R c h}=0.0001$ ) of $\alpha$-tubulin in fixed Vero cells, based on 6 sub-images for different pattern orientations. The pixel size is set to $p x=20 \mathrm{~nm}$, the excitation power to $P_{\text {exc }}=2.5 \mu \mathrm{W}$ and the STED laser power to $P_{\text {STED }}=32 \mathrm{~mW}$. The pixel dwell time is $p T=2 \mu$ s per sub-image, resulting in a total effective pixel dwell time of only $12 \mu \mathrm{s}$. The scale bar indicates a length of $2 \mu \mathrm{m}$. (c) shows a line profile of a filament (averaged over 5 neighboring lines), yielding the same resolution enhancement as in figure 4.4

duction of the pixel dwell time to $2 \mu$ s per sub-image. In this way, a total pixel dwell time of $12 \mu$ s can be realized, which is considerably less than typically employed under similar imaging conditions (cf. subsection 4.2.2). Thus, this yields a tomoSTED acquisition at a significantly reduced light dose compared to the 2D STED acquisition. Exemplary results on microtubules are displayed in figure 4.5. Figure 4.5 (a) shows a confocal scan and figure 4.5(b) the respective tomoSTED image. Six different pattern orientations at a pixel dwell time of $2 \mu$ s each are switched on a pixel level and reconstructed employing the Richardson-Lucy deconvolution. The color bar is chosen individually for each image to cover the full dynamic range, while the scale bar is the same for both images and indicates a length of $2 \mu \mathrm{m}$. A line profile, averaged over 5 neighboring lines, is displayed in figure 4.5(c) and indicates the same image FWHM as for the results presented in figure 4.4. Consequently, tomoSTED microscopy enables the acquisition of super-resolved images of uncompromised quality at a significantly reduced depletion light dose for a pixel-wise switching of the depletion pattern orientation.

\subsection{FastRESCue}

TomoSTED microscopy has successfully proven its ability to reduce the light dose imposed on the sample by a factor of 4 Krü17. As discussed in section 2.3, several other techniques have been developed in the recent years in order to minimize photodamage, with RESCue [SER ${ }^{+} 11$. being among them. This section is dedicated to the implementation of the novel FastRESCue 
technique (see section 3.5), which enhances the performance of RESCue by not only lowering the light dose, but also reducing the acquisition time necessary to obtain a high-resolution image. First, the performance of the scan system introduced in section 3.5 is compared to the one of the conventional scan via the Imspector software in order to guarantee comparability of the results. Second, FastRESCue applications on both fluorescent microspheres as well as various cellular structures are presented.

Being an evolution of RESCue, FastRESCue can obviously be combined with any imaging technique for which RESCue is applicable. Thus, both tomoSTED and 2D STED microscopy can take advantage by the novel features introduced by this technique. A 1D depletion pattern is the ideal candidate for the characterization of the scan system, since potential artifacts or jitters are better visible for the elongated 1D STED PSF. However, to ensure a direct comparison with the RESCue applications demonstrated in literature during the past years, conventional 2D STED microscopy is employed for most of the imaging part.

Please note that all further measurements within this thesis utilizing the novel scan system are performed with self-written LabVIEW routines and the resulting images are acquired without any pulse gating.

\subsubsection{Characterization of the EOD-assisted scan system}

In this subsection, the scan system composed of the two electro-optic deflectors in combination with a galvanometer scanner is analyzed and the outcome is compared to scans performed conventionally via the Imspector software.

A crucial experimental requirement is the positioning of the EODs in such a way that the pivot point lies in a plane which is conjugated to the objective's back focal plane. In this way, the beam only experiences a tilt in this plane while scanning, resulting in a pure displacement without tilt in the sample plane. In order to verify the correct alignment, a scan is performed utilizing only the EODs as scanning device. The measurement is conducted for two orientations of a 1D depletion pattern, corresponding to the two scan axes of the system. The resulting STED images are displayed in figure 4.6 (c,d) in comparison to the conventional scan employing the galvanometer scanner (figure 4.6 (a,b)). The distortions visible at the edges of the images in figure 4.6(c,d) are due to the lack in scan range and show that the full scan range of the EODs is slightly smaller than the field of view shown here, as also theoretically predicted. However, in the central region of the image, corresponding to a scan range of approximately $\pm 1 \mu \mathrm{m}$, the homogeneous image quality confirms the correct positioning of the EODs.

Subsequently, the EODs are combined with the galvanometer scanner as described in subsection 3.5.3. Scans of fluorescent microspheres for a $1 \mathrm{D}$ depletion pattern are acquired conventionally (i.e. by means of a galvanometer scanner and the Imspector software) as well as utilizing the self-written LabVIEW program with the FPGA. The orientation of the 1D de- 

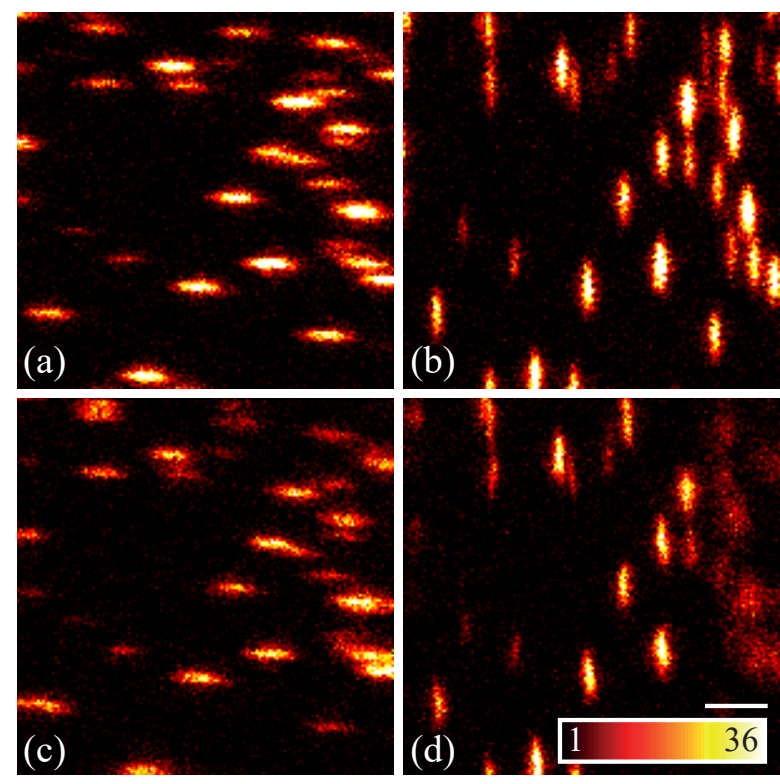

Figure 4.6: Comparison of different scanning devices employed for 1D STED images of Crimson 40 microspheres: (a,b) Conventional scan with a galvanometer scanner controlled via the Imspector software for two different orientations of the STED depletion pattern. The pixel size is set to $p x=20 \mathrm{~nm}$, the pixel dwell time to $p T=30 \mu \mathrm{s}$, the excitation power to $P_{\text {exc }}=5.5 \mu \mathrm{W}$ and the STED laser power to $P_{\mathrm{STED}}=50 \mathrm{~mW}$. (c,d) Scan with the electro-optic deflectors for the same acquisition parameters. The same color bar is chosen for all images for an easier comparison. The scale bar indicates a length of $500 \mathrm{~nm}$.

pletion pattern is chosen such that the high-resolution axis and the fast scan axis coincide for a higher visibility of eventual distortions. The conventional scan is displayed in figure 4.7(a) with a closeup shown in figure 4.7(d). To distinguish potential effects caused by the FPGA scan and effects caused by the combined scan system, a scan with the FPGA program, but without EODs is conducted. The results are shown in figure 4.7(b) and (e), respectively. Line profiles, averaged over 10 neighboring lines, are drawn over a single microsphere and compared in figure $4.7(\mathrm{~g})$. Gaussian fits yield a FWHM of $(84.1 \pm 2.6) \mathrm{nm}$ for the conventional scan and $(80.7 \pm 2.0) \mathrm{nm}$ for the FPGA scan and practically the same peak intensity for both. Implementing the EODs in the measurements yields the results displayed in figure 4.7(c) and (f), respectively. From the line profile, a displacement between this measurement and the two previous measurements is visible. This displacement is caused by the mass inertia of the galvanometer scanner (see figure 3.12 and is present in every image scan. As this displacement is constant over the whole field of view for constant pixel dwell times, it is usually not visible. The EODs compensate for this displacement between nominal and actual scan position.

Noticeably, the FWHM and peak intensity obtained for the scan with FPGA and EODs is unaltered compared to the scan without EODs and moreover to the conventional Imspector scan. Furthermore, no distortions or artifacts are visible over the whole field of view covering $40 \mu \mathrm{m}$ in the direction of the fast scan axis. Consequently, the combined scan system shows 
the same performance as the conventional scan system for a classical image acquisition.

The same measurement can be conducted switching the fast axis of the scanner in order to also verify the correct alignment of the second EOD. Similar results are obtained (data not shown).

Finally, the new scan system with EODs is employed for RESCue measurements. For a first comparison, the same 1D depletion pattern as for the previous measurement (figure 4.7) is utilized. All scans are performed with the FPGA and various RESCue modalities are studied. Figure 4.8(a) shows a scan without RESCue, with a closeup displayed in figure 4.8(d). As before, line profiles of a single microsphere are compared for all imaging modalities in order
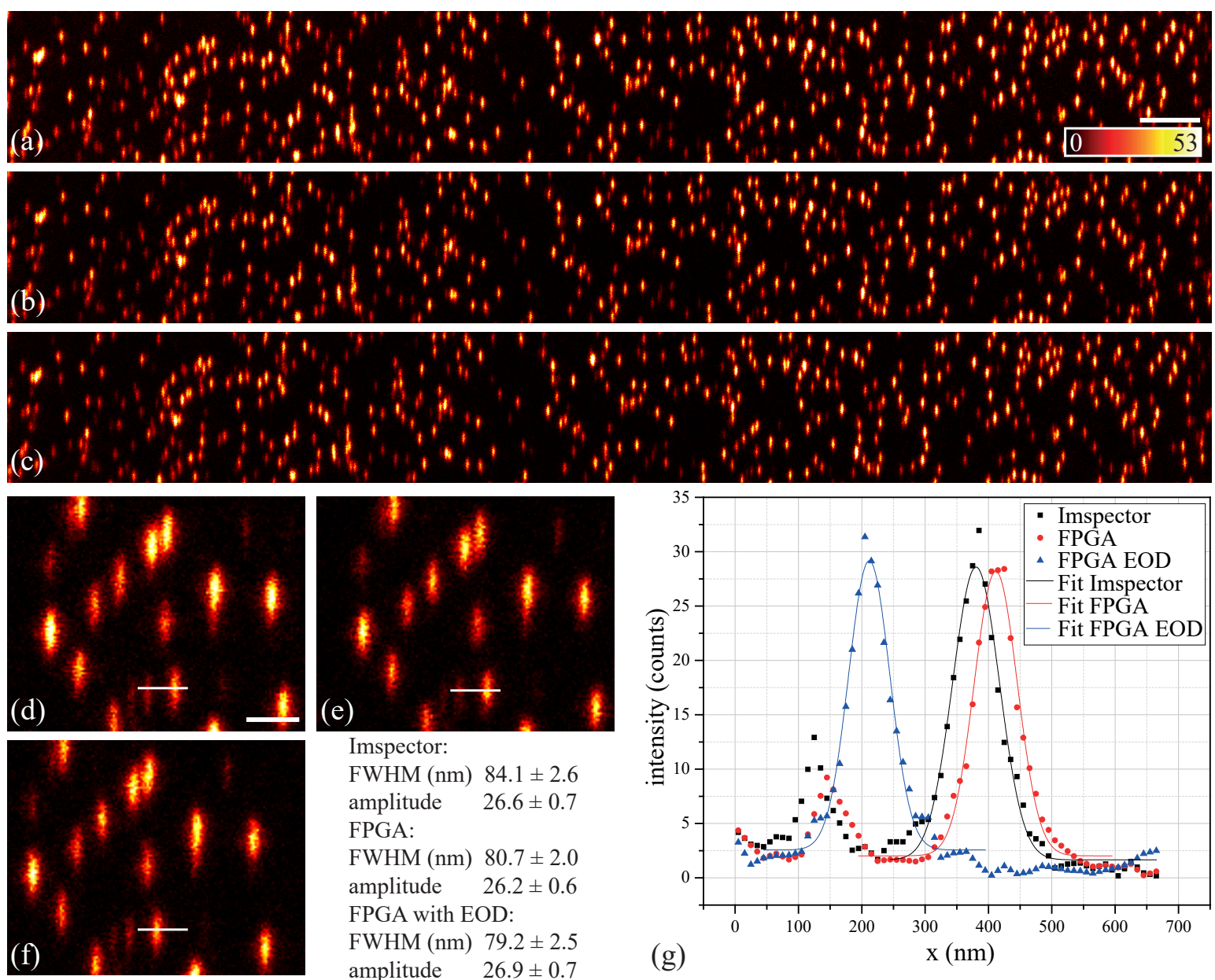

Imspector:

FWHM (nm) $84.1 \pm 2.6$ amplitude $\quad 26.6 \pm 0.7$ FPGA:

FWHM (nm) $80.7 \pm 2.0$ amplitude $\quad 26.2 \pm 0.6$ FPGA with EOD:

FWHM (nm) $79.2 \pm 2.5$ amplitude $\quad 26.9 \pm 0.7$

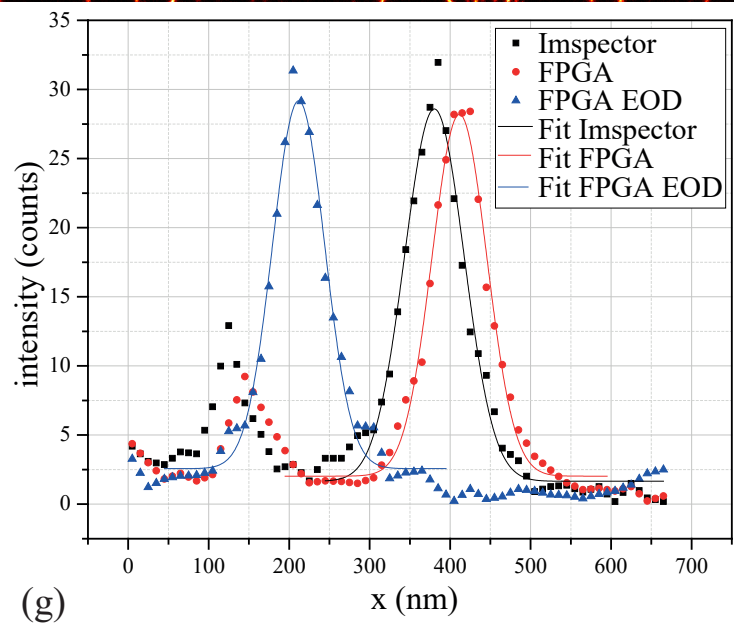

Figure 4.7: Comparison of different scan modes for STED imaging of Crimson 40 microspheres for a 1D STED depletion pattern: (a) Conventional scan with the Imspector software for $p x=20 \mathrm{~nm}$, $p T=30 \mu \mathrm{s}, P_{\text {exc }}=9.3 \mu \mathrm{W}$ and $P_{\mathrm{STED}}=34 \mathrm{~mW}$. (b,c) Scan with the LabVIEW routine via the FPGA (b) without and (c) with the EODs for compensating the offset between intended and actual scan position introduced by the galvanometer scanner. The same color bar is chosen for all images, the scale bar indicates a length of $2 \mu \mathrm{m}$. (d-f) Closeups of the images (a-c) with the scale bar indicating $500 \mathrm{~nm}$. (g) Line profiles over a single microsphere (averaged over 10 neighboring lines) as drawn in (d-f) are compared and fitted by a Gaussian distribution. The fit results for FWHM and amplitude are stated for comparison. 
to judge potential changes in resolution or signal count. They are displayed in figure $4.8(\mathrm{~h})$ in combination with corresponding Gaussian fits. A conventional RESCue scan with an upper threshold of $u T h=30$ counts and a lower threshold of $l T h=2$ counts in $d T=25 \%$ of the total pixel dwell time yields a resultant light dose of $36 \%$ compared to the conventional image and is displayed in figure 4.8 (b) and (e), respectively. The FWHM of the exemplary
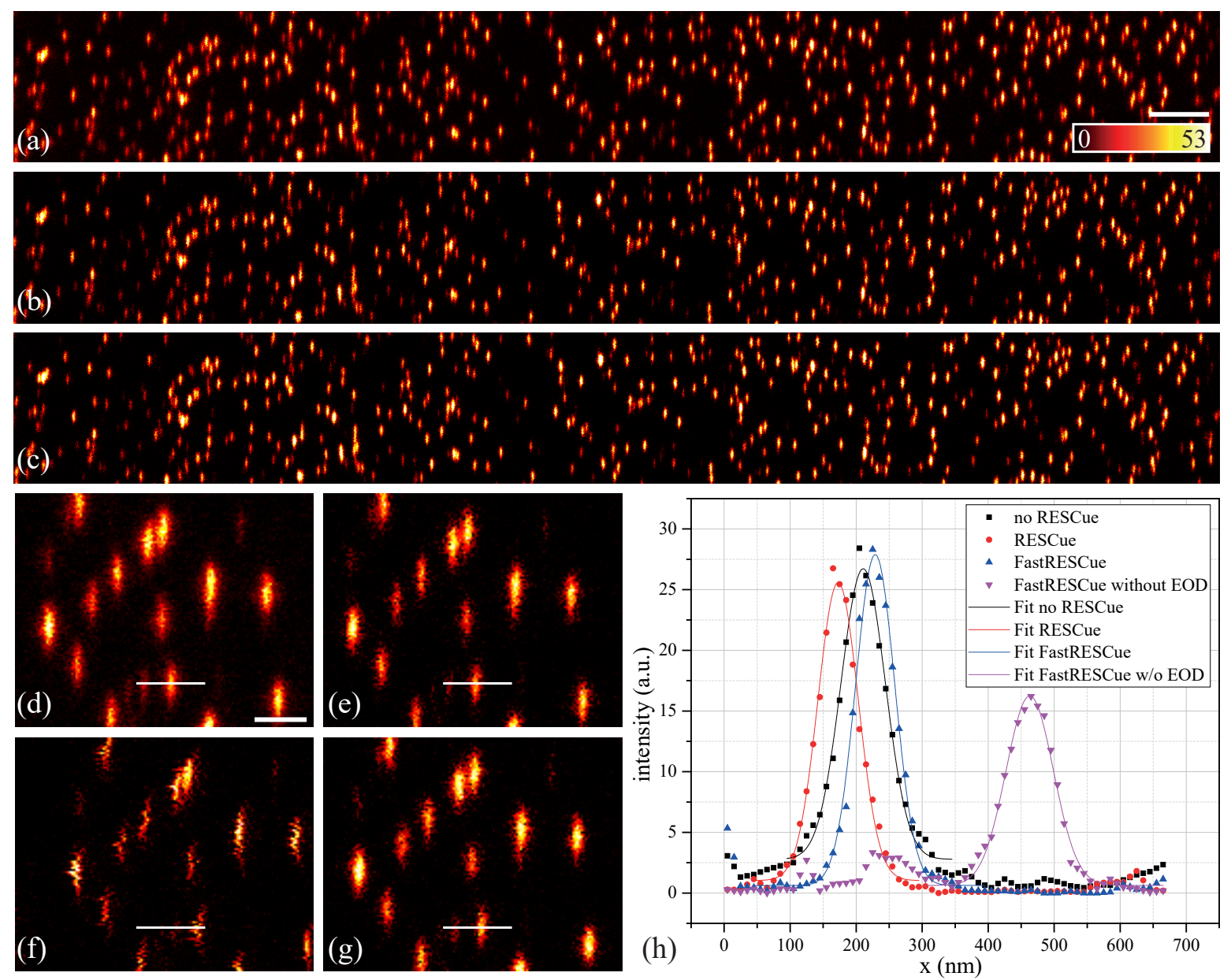

Figure 4.8: Comparison of different RESCue modes for STED imaging on Crimson 40 microspheres for a 1D STED depletion pattern. All scans are performed with the FPGA for $p x=20 \mathrm{~nm}, p T=30 \mu \mathrm{s}$, $P_{\text {exc }}=9.3 \mu \mathrm{W}$ and $P_{\text {STED }}=34 \mathrm{~mW}$. (a) Scan without RESCue. (b) Scan with conventional RESCue for $u T h=30, l T h=2, d T=25 \%$, resulting in a light dose of $36 \%$ compared to the scan in (a). (c) FastRESCue scan for the same RESCue parameters as in (b). The scale bar for (a-c) indicates a length of $2 \mu \mathrm{m}$, the same color table is chosen for all three images. (d,e,g) show closeups (scale bar $500 \mathrm{~nm}$ ) of (a,b,c) with line profiles over a single microsphere averaged over 10 neighboring lines. (f) is a closeup of the same area for a scan performed with FastRESCue, but without the EODs for positioning correction. A line profile is drawn as well, with the respective data shown in (h). Gaussian fits allow the comparison of resolution and amplitude. The fit results are given as FWHM $=(79.3 \pm 2.3) \mathrm{nm}$, amplitude $=23.9 \pm 0.5$ for the scan without RESCue, FWHM $=(72.0 \pm 1.9) \mathrm{nm}$, amplitude $=24.5 \pm 0.5$ for standard RESCue, FWHM $=(70.0 \pm 1.5) \mathrm{nm}$, amplitude $=27.3 \pm 0.5$ for FastRESCue with EODs and FWHM $=(84.6 \pm 2.1) \mathrm{nm}$, amplitude $=15.6 \pm 0.3$ for the scan with FastRESCue, but without EODs. 
line profile for the RESCue scan is slightly lowered compared to the conventional scan due to the better structure detection by the suppression of background, as can be already seen from the raw data displayed in figure $4.8(\mathrm{~h})$. The image quality of both scans is comparable over the whole field of view and no artifacts due to the extrapolation of signal for bright pixels are visible. Ultimately, a FastRESCue acquisition of the same field of view with the same RESCue parameters is performed and shown in figure 4.8(c) and (g) for the whole field of view and the closeup, respectively. It exhibits the same image quality as the scan without RESCue and with conventional RESCue. Moreover, both the FWHM as also the peak intensity are comparable to the respective quantities in the previous scans. The necessity of the EODs for this acquisition is illustrated by figure 4.8(f): A closeup of a FastRESCue scan without EODs (large field of view not shown) exhibits clear distortions. These are caused by the mass inertia of the scanner, which leads to a changing displacement for a varying pixel dwell time. Consequently, FWHM and peak intensity (cf. figure 4.8 (h)) are significantly worse than the beforehand presented results.

In conclusion, the novel scan system exhibits the same performance as the conventional scan via the Imspector software for standard image acquisition both without and with the correction of the scan position accomplished by the EODs. Moreover, the EOD correction has proven strictly necessary for the implementation of FastRESCue, which has been shown to yield the same image quality and resolution at lower light dose and acquisition time. It can hence be applied for imaging biological structures as conducted in the following.

\subsubsection{FastRESCue for imaging}

The potential of FastRESCue for reducing both light dose and acquisition time is demonstrated on both fluorescent microspheres and on several biological structures.

FastRESCue is first applied for tomoSTED images of fluorescent microspheres for a pixel-wise switched pattern orientation and compared to an acquisition without RESCue. Six different pattern directions are recorded, with two exemplary directions displayed in figure $4.9(\mathrm{a}, \mathrm{b})$ for the measurement without RESCue and in figure 4.9(c,d) for the FastRESCue measurement. A comparable image quality can be observed for all four images, as expected from the measurements presented in subsection 4.2.1. This leads to a comparable image quality also for the reconstructed images in figure $4.9(\mathrm{~g}, \mathrm{~h})$. The RESCue parameters have been chosen as $u T h=30, l T h=2$ and $d T=25 \%$, resulting in an overall light dose of $32 \%$ for the FastRESCue acquisition as compared to the acquisition without RESCue. The resulting pixel dwell times for each pixel are displayed in figure $4.9(\mathrm{e}, \mathrm{f})$ for the two orientations. Please note that these dwell times are not consequently identical for all pattern orientations. The overall frame time of the FastRESCue image amounts to $75 \%$ of the overall frame time of the acquisition without RESCue, which is significantly larger than expected from the light dose. This difference originates in a wait time implemented to allow for a full switching of the pattern orientation. Due 

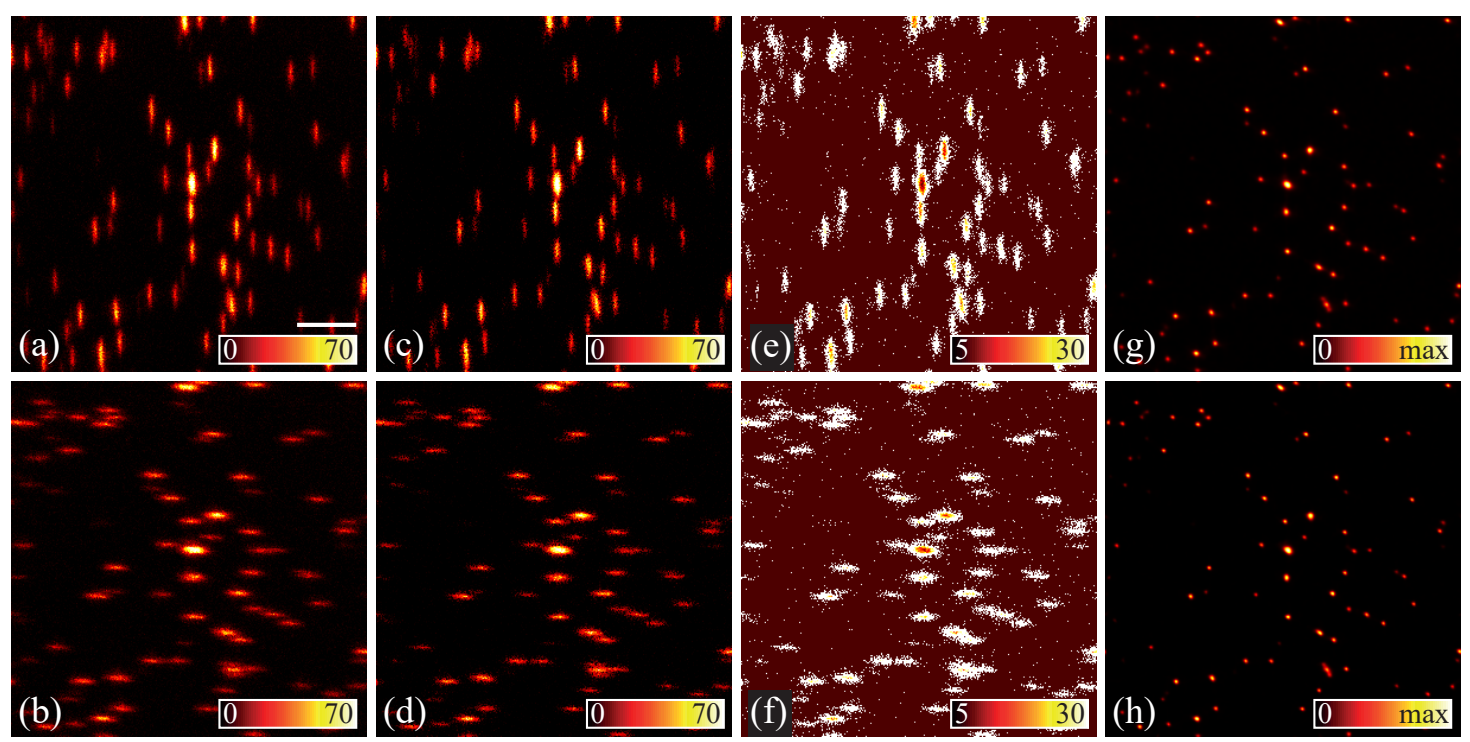

Figure 4.9: FastRESCue for tomoSTED imaging of Crimson 40 microspheres. For $p x=20 \mathrm{~nm}$, $p T=30 \mu \mathrm{s}$ per direction, $P_{\mathrm{exc}}=9.3 \mu \mathrm{W}$ and $P_{\mathrm{STED}}=50 \mathrm{~mW}$, tomoSTED images for 6 pattern orientations are recorded (a,b,g) without RESCue and (c,d,h) with FastRESCue. (a-d) show exemplary sub-images for two pattern orientations, whereas $(\mathrm{g}, \mathrm{h})$ show the reconstructed images, taking into account all 6 recorded sub-images. The Richardson-Lucy deconvolution is performed with 15 iteration steps and $\alpha_{R c h}=0.0001$. (e,f) The pixel dwell time (in $\mu \mathrm{s}$ ) for each pixel is graphically displayed for the FastRESCue acquisition and the two orientations shown in $(\mathrm{c}, \mathrm{d})$. The RESCue parameters are set as $u T h=30, l T h=2$ and $d T=25 \%$, resulting in an overall light dose of $32 \%$ compared to the acquisition without RESCue. A wait time of $50 \mu \mathrm{s}$ is included between the individual pixel acquisitions to ensure a complete switching of the pattern orientations for both the scan without RESCue and the scan with FastRESCue. The frame time in the scan with FastRESCue is consequently not much lower than in the scan without RESCue and amounts to $75 \%$ thereof. The scale bar indicates a length of $1 \mu \mathrm{m}$ in all images.

to limitations in the actual high-voltage amplifiers' technology, a switching is only guaranteed in the time span of $50 \mu \mathrm{s}$. The wait time is constant for both acquisition modes and therefore highly influences the overall frame time. This limitation can be obviously circumvented by employing a line- or image-wise switching of the pattern orientation, making the combination of tomoSTED microscopy and FastRESCue an attractive and feasible choice.

For an adequate comparison of FastRESCue and RESCue as presented in [SER $\left.{ }^{+} 11\right]$, a doughnutshaped depletion intensity distribution is employed for the further measurements. It is also generated utilizing conical diffraction by choosing circular polarization for all three Pockels cells pairs as outlined in [Bio14] and Per13]. A representative STED PSF, visualized by imaging gold beads, is displayed in the inset of figure 4.10 (b).

The light dose saving for RESCue is essentially limited by the lower threshold for sparse samples. Due to background and dead counts of the detector, false positive pixel, i.e. pixels which exhibit a photon count despite the absence of structure, are relatively common. This aspect can be tackled by introducing a second lower threshold $l T h_{2}, d T_{2}$ with $l T h_{2}>l T h_{1}$ and $d T_{2}>d T_{1}$. 
In this way, the statistical nature of fluorescence emission can be exploited to detect such false positive pixel and stop the illumination after $d T_{2}$ instead of illuminating for the whole pixel dwell time $p T$.

Preliminary experiments are conducted on fluorescent microspheres again, for which an image acquisition is performed for both confocal and 2D STED microscopy without RESCue, further referred to as standard STED microscopy. The results are displayed in figure 4.10(a,b), respectively, with the inset in figure 4.10(b) showing the STED PSF measured by imaging reflective gold beads. The corresponding FastRESCue STED image is shown in figure 4.10.(c) and exhibits the same image quality as the reference in figure 4.10(b). This confirms the correct setting of the RESCue parameters, which are chosen as $u T h=50, l T h_{1}=1, l T h_{2}=2, d T_{1}=8 \%$ and $d T_{2}=12 \%$ in this case. The resultant light dose is 6 -times lower, amounting to $15.9 \%$ of the light dose necessary for the standard acquisition. The acquisition time for the frame shown in figure 4.10.(c) equals $17.8 \%$ compared to the respective quantity in figure 4.10(b). To judge the decisions taken by the imaging algorithm, the map of the effective pixel dwell times is depicted in figure 4.10.(d). As immediately apparent, both in areas without structure as well as in areas of high signal, time and light dose can be saved by applying FastRESCue. Additionally, few background pixel are detected as false-positive, indicating a not too restrictive setting of the lower thresholds, which is important to avoid overlooking of dim structures.

As seen from the example of fluorescent microspheres, the resultant light dose (given in percent of the light dose employed for standard acquisition), further denoted as 'relative FastRESCue light dose', is slightly lower than the resultant frame time (also in percent of the standard ac-
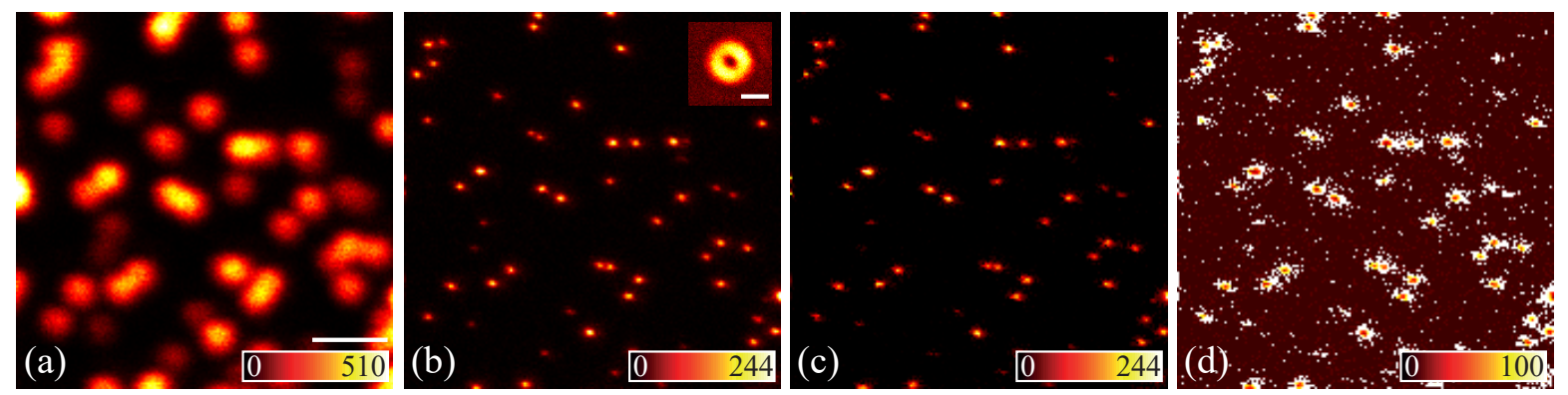

Figure 4.10: FastRESCue reduces light dose and frame time by a factor of 6 : Images of fluorescent microspheres of $48 \mathrm{~nm}$ diameter at a pixel size of $p x=30 \mathrm{~nm}$ and a pixel dwell time of $p T=100 \mu$ s for (a) confocal, (b) standard STED and (c) FastRESCue acquisition (scale bar $1 \mu \mathrm{m}$ ). The inset (b) depicts the STED PSF measured on reflective, $150 \mathrm{~nm}$-sized gold beads (scale bar $500 \mathrm{~nm}$ ). The excitation power is set to $P_{\text {exc }}=12.9 \mu \mathrm{W}$ and the STED laser power to $P_{\mathrm{STED}}=80 \mathrm{~mW}$ for all conditions. The RESCue parameters are chosen as $u T h=50, l T h_{1}=1, l T h_{2}=2, d T_{1}=8 \%$ and $d T_{2}=12 \%$, resulting in a light dose of $15.9 \%$ and a frame time of $17.8 \%$ compared to the standard acquisition. (d) shows the effective pixel dwell times, illustrating the potential to speed up acquisition both in areas without structure as well as in areas of high signal. 
quisition time), denoted as 'relative FastRESCue frame time'. The remaining difference results from the overhead of the LabVIEW routine as well as on a line fly-back time for the scanner: For fields of view larger than the maximum scan range of the EODs, a wait time is implemented at the end of each line to allow the scan system to go back to the beginning of the line. As this wait time and the overhead of the program are a constant for both the standard and the FastRESCue acquisition, the saving in frame time is slightly less than the saving in light dose. This dependence is analyzed in more detail by imaging fluorescent microspheres for varying RESCue parameters, yielding different light dose and frame time savings. The resultant relative FastRESCue light dose and frame time per image are calculated and their dependence is displayed in figure 4.11

The measured data for the relative FastRESCue frame time and light dose are close to the bisectrix, i.e. the curve of equal relative FastRESCue frame time and light dose, which is visualized in red as reference. The latter is slightly lower than the measured data, with the difference between relative FastRESCue light dose and frame time increasing for lower light dose. This difference depends on the chosen maximal pixel dwell time as well as on the constant overhead of the program, which is more dominant for smaller times. Nevertheless, it is shown that the light dose saved by conventional RESCue directly translates into a faster acquisition for FastRESCue imaging. Consequently, the advantages of FastRESCue can immediately be

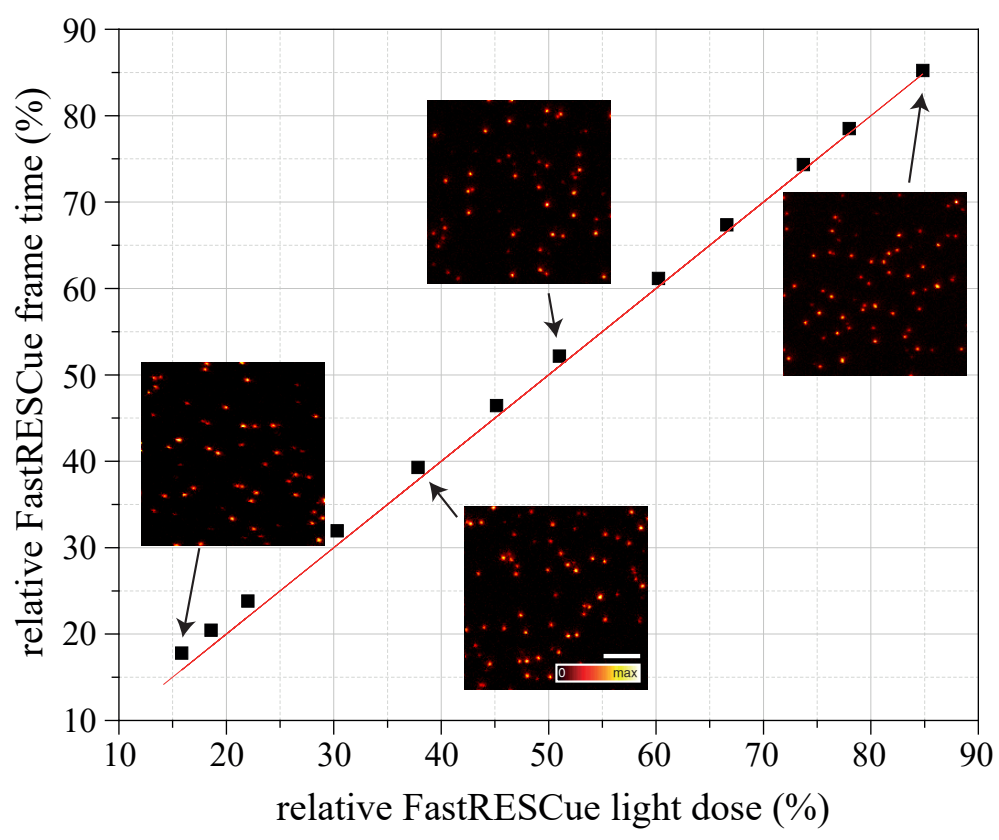

Figure 4.11: The light dose saved by conventional RESCue directly translates into a faster acquisition for FastRESCue imaging: Fluorescent microspheres are imaged for various RESCue parameters, and the resultant light dose and frame time compared to the standard acquisition is depicted. The acquisition parameters are chosen as for Fig. 4.10 the scale bar in the example images equals $1 \mu \mathrm{m}$. The red curve visualized equal light dose and frame time saving. 
exploited in all applications for which RESCue is advantageous.

Since RESCue is typically employed for imaging biological structures, in the following FastRESCue results for various biological structures are presented. Primarily, the advantages of FastRESCue are analyzed for fluorescently labeled microtubules in Vero cells, as displayed in figure 4.12. The FastRESCue image in figure 4.12(b) shows the same image quality as the standard acquisition in figure 4.12 (a), at only $22.7 \%$ of the frame time. The detailed distribution of pixel dwell times in figure 4.12(c) corresponds well with areas of high or very low signal (i.e. stained structure or background).

To analyze the advantage of FastRESCue on all scales of magnitude, the image in figure 4.12(b) is divided in disjoint quadratic sub-ROIs of a certain size. The light dose per sub-ROI relative to the equivalent sub-ROI in the original image in figure 4.12 (a) is calculated by the sum of the respective pixel dwell times. The size of the sub-ROIs is varied and the local light dose (relative to the standard acquisition) is displayed as a histogram as shown in figure 4.12(d) for four different sub-ROI sizes. For the histogram, intervals of $5 \%$ are chosen, i.e. events between 0 and $5 \%$ relative FastRESCue light dose are summed up and displayed at $2.5 \%$. The variance of this distribution is displayed in figure 4.12 (e) as a function of the sub-ROI size.
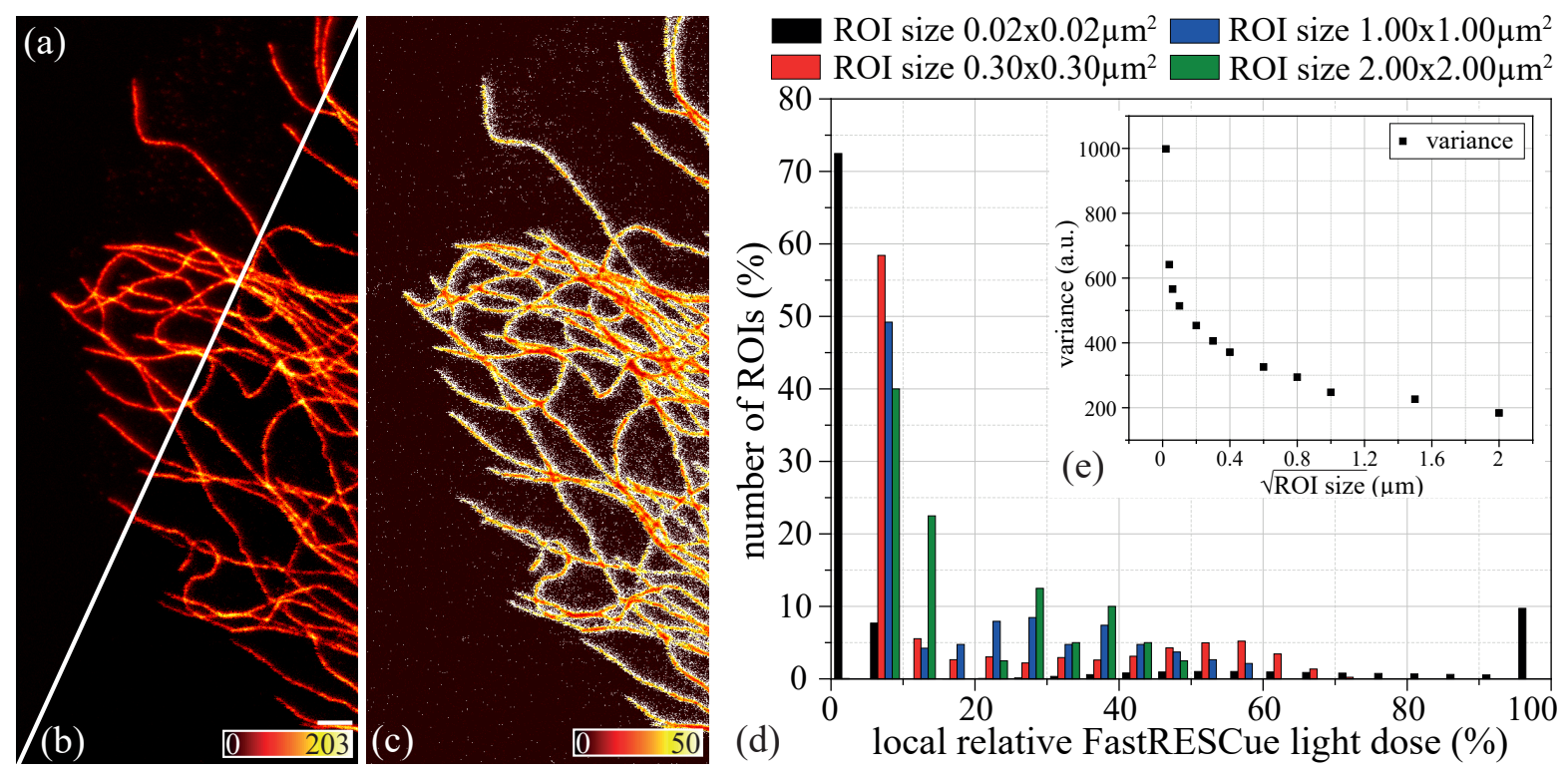

Figure 4.12: Analysis of the advantage of FastRESCue depending on the size scale: Images of $\alpha$-tubulin in Vero cells (dye: Abberior STAR RED) for (a) standard STED and (b) FastRESCue acquisition with $P_{\mathrm{exc}}=5.3 \mu \mathrm{W}, P_{\mathrm{STED}}=57 \mathrm{~mW}, p x=20 \mathrm{~nm}, p T=50 \mu \mathrm{s}, u T h=40, l T h_{1}=1$ and $l T h_{2}=2, d T_{1}=5 \%$ and $d T_{2}=9 \%$ (scale bar $\left.1 \mu \mathrm{m}\right)$. The global light dose amounts to $20.3 \%$ for a frame time of $22.7 \%$ for the FastRESCue acquisition (b) compared to the standard acquisition (a). The distribution of the effective pixel dwell time $(c$, in $\mu \mathrm{s})$ is analyzed in detail by dividing the image in disjoint sub-ROIs of varying size. The local relative FastRESCue light dose of all sub-ROIs of a specific size is statistically analyzed. Histograms for different ROI sizes (with edge length 1,15,50 and 100 pixel) are displayed in (d), with the variance as a function of the ROI size being depicted in (e). 
For increasing sub-ROI size, a decrease of the distribution's variance can be observed: The distribution itself, as seen from figure 4.12(d), shows two peaks at $2.5 \%$ and $97.5 \%$ for a sub-ROI size of one pixel, i.e. $20 \times 20 \mathrm{~nm}^{2}$, corresponding to the first lower threshold and the maximum pixel dwell time, respectively. The events at $7.5 \%$ correspond to the second lower threshold, and all other events reflect the pixels where the acquisition was stopped due to the upper threshold. For increasing sub-ROI size, the outer peaks vanish due to the natural inhomogeneities in the structure distribution, as seen from figure 4.12(b). Nevertheless, also for large
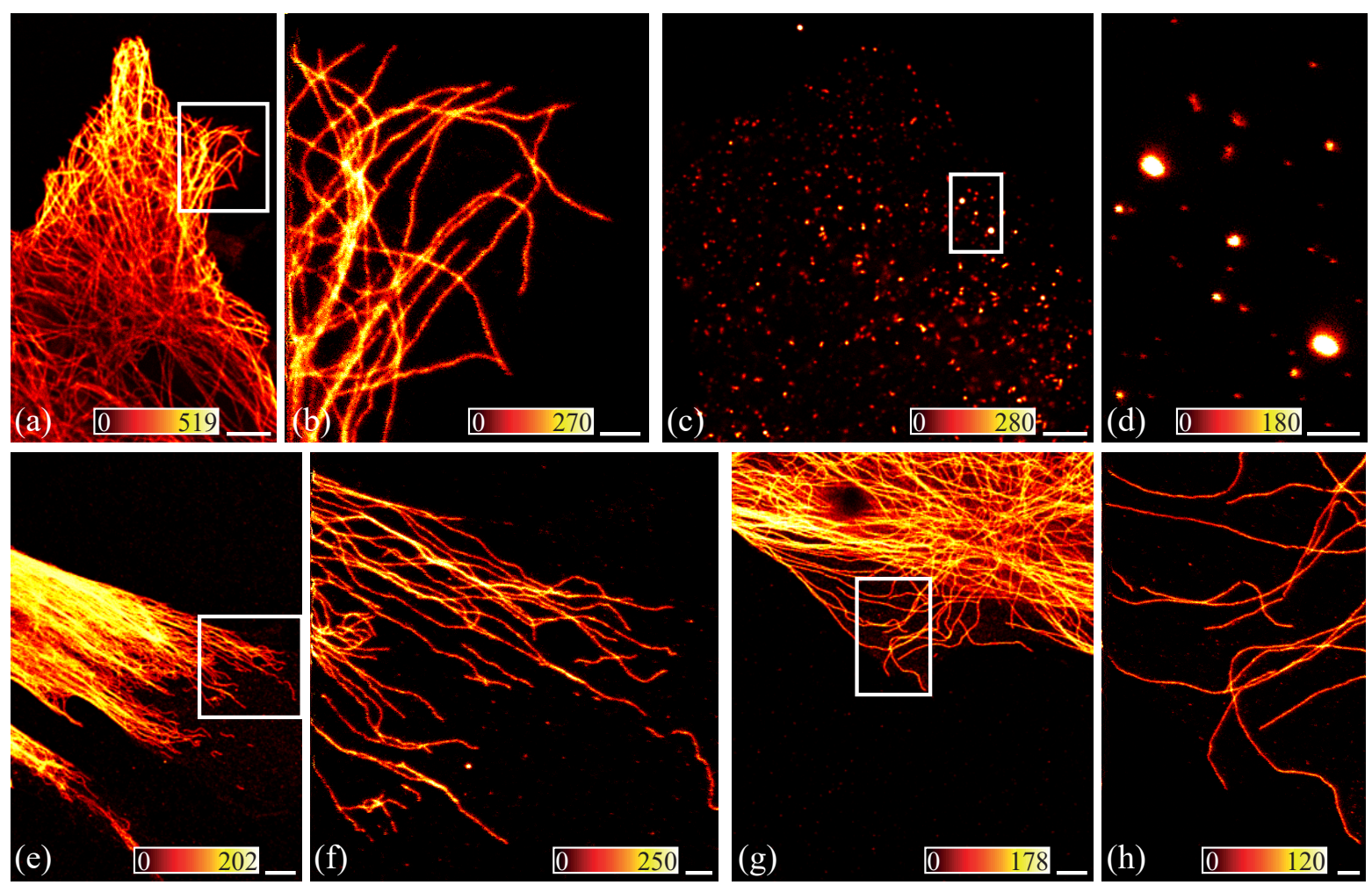

Figure 4.13: Images of various cellular structures: (a,b) Fluorescently labeled $\alpha$-tubulin in Vero cells (dye: Abberior STAR RED). The acquisition parameters for the confocal overview (a) are chosen as $P_{\text {exc }}=2.12 \mu \mathrm{W}, p x=100 \mathrm{~nm}$ and $p T=30 \mu \mathrm{s}$. (b) The FastRESCue acquisition is performed with $P_{\text {exc }}=5.3 \mu \mathrm{W}, u T h=40, d T_{1}=4 \%$ and $d T_{2}=7 \%$. The light dose amounts to $22.1 \%$ for a frame time of $24.4 \%$ compared to the standard STED acquisition. (c,d) Fluorescently labeled peroxisomes (PMP70) in Vero cells (dye: Abberior STAR 635P) for $P_{\text {exc }}=2.12 \mu \mathrm{W}, p x=100 \mathrm{~nm}$ and $p T=30 \mu \mathrm{s}(\mathrm{c})$. (d) For FastRESCue, the parameters are chosen as $P_{\text {exc }}=8.2 \mu \mathrm{W}, u T h=50, d T_{1}=10 \%$ and $d T_{2}=$ $16 \%$, resulting in a light dose of $15.8 \%$ and a frame time of $19.0 \%$. (e,f) Fluorescently labeled vimentin in fibroblasts (dye: Abberior STAR 635P). (e) Confocal overview $\left(P_{\text {exc }}=3.3 \mu \mathrm{W}, p x=200 \mathrm{~nm}\right.$ and $p T=10 \mu \mathrm{s}$ ) and (f) FastRESCue acquisition with $17.5 \%$ of light dose and $19.8 \%$ frame time compared to the standard acquisition. The parameters for the latter are chosen as $P_{\text {exc }}=16.6 \mu \mathrm{W}, u T h=40$, $d T_{1}=4 \%$ and $d T_{2}=7 \%$. (g,h) Fluorescently labeled $\alpha$-tubulin in fibroblasts (dye: Abberior STAR 635P): The acquisition parameters are set to $P_{\mathrm{exc}}=2.12 \mu \mathrm{W}$ for $p x=200 \mathrm{~nm}, p T=10 \mu \mathrm{s}$ for the confocal overview (g) and $P_{\text {exc }}=3.3 \mu \mathrm{W}, u T h=40, d T_{1}=10 \%$ and $d T_{2}=15 \%$ for the FastRESCue acquisition (h). This enables a resultant light dose of $22.5 \%$ and frame time of $24.4 \%$ compared to the standard acquisition. If not otherwise stated, $P_{\mathrm{STED}}=57 \mathrm{~mW}, p x=20 \mathrm{~nm}, p T=50 \mu \mathrm{s}, l T h_{1}=1$ and $l T h_{2}=2$. The scale bar is given by $5 \mu \mathrm{m}(\mathrm{a}, \mathrm{c}, \mathrm{e}, \mathrm{g})$ and $1 \mu \mathrm{m}(\mathrm{b}, \mathrm{d}, \mathrm{f}, \mathrm{h})$. 
sub-ROI sizes, the main peak remains below 10\%, implying that, while a global improvement of a factor of 5 can be successfully realized, FastRESCue is actually even able to locally speed up the acquisition up to 10 times.

FastRESCue acquisitions of various representative biological structures are displayed in figure 4.13 for various cell types, resulting in a relative FastRESCue frame time between 19\% and $25 \%$. For each image, a confocal overview of the cell, highlighting the area chosen for the FastRESCue STED acquisition, is shown as well. Figure 4.13 (a,b) is a second example for fluorescently labeled microtubules in Vero cells and shows a similar advantage as in figure 4.12. The RESCue parameters have been slightly modified and found to be optimal as $u T h=40$, $d T_{1}=4 \%, d T_{2}=7 \%$ and $p T=50 \mu$ s for this field of view.

Figure 4.13(c,d) shows the fluorescently labeled membrane of peroxisomes in Vero cells fixed with PFA. Despite of the huge variations in size and thus signal, also dim structures are correctly recognized and imaged. In order to ensure this, the lower thresholds have been chosen much higher $\left(d T_{1}=10 \%, d T_{2}=16 \%\right.$ and $\left.p T=50 \mu \mathrm{s}\right)$ compared to the previous example, yielding the optimal trade-off between speed gain and image quality. The color table is chosen appropriately to enable the visibility of all structures.

In figure 4.13(e,f), fluorescently labeled vimentin in fibroblasts is displayed both in the confocal overview as well as in the FastRESCue acquisition. The RESCue parameters have been chosen similarly to figure 4.13(a,b). Despite the more restrictive lower threshold compared to figure 4.13 (c,d), also dimmer structures visible in the background can be correctly resolved. Finally, figure $4.13(\mathrm{~g}, \mathrm{~h})$ shows fluorescently labeled microtubules in fibroblasts. The FastRESCue image in figure 4.13( $\mathrm{h}$ ), at a frame time of $24.4 \%$ compared to the standard acquisition, shows no artifacts or distortions. The RESCue parameters were set to $u T h=40, d T_{1}=10 \%$, $d T_{2}=15 \%$ and $p T=50 \mu$ s to allow the detection of both dim and very bright structures.

\subsection{Filament tracing}

Within this work, two techniques for imaging at a reduced light dose at an increased acquisition speed compared to conventional 2D STED microscopy, namely tomoSTED microscopy and FastRESCue STED microscopy, have been discussed in detail. The former is based on an optimization of the depletion pattern, while the latter aims at reducing the average pixel dwell time.

A further technique for an increased acquisition speed can be realized by filament tracing algorithms, where not a whole, pre-defined field of view is imaged, but the scan pattern is adapted on-line based on the acquired signal. Such a system for the tracing of filamentous structures based on tomoSTED microscopy relies on a fast switching of the pattern orientation, as presented in section 3.2 , an adaptive scan system, developed in the course of section 3.5 , and a suitable decision algorithm. The latter is developed and presented within this section, 
with a coarse draft of the LabVIEW implementation already discussed in subsection 3.5.4. The first part of this section (see subsection 4.3.1) is dedicated to results on simulated structures, with the important aspects of the algorithm being analyzed. In the second part, experimental data on actin in vitro (see subsection 4.3.2) and microtubules in fixed (see subsection 4.3.3) and living fibroblasts (see subsection 4.3.4) are presented.

\subsubsection{Tracing of simulated structures}

To develop an algorithm for the tracing of filamentous structures, suitable criteria for the determination of a filament's position and direction need to be identified. 1D depletion patterns yielding a one-dimensional resolution increase seem to be the method of choice for imaging a filamentous structure, which itself only shows a FWHM below the diffraction limit perpendicular to the structure direction. To verify their suitability, 1D STED images of a ring-like structure are simulated for 1D depletion patterns of various orientations (see figure 4.14(a-c,e-g)). By the choice of a circle as underlying structure, all possible structure orientations are considered, enabling a comprehensive analysis of suitable criteria. All simulations within this subsection are performed with self-written MATLAB (MathWorks, USA) code.

For an on-line adaption of the scan pattern, only the signal acquired at previous scan posi-
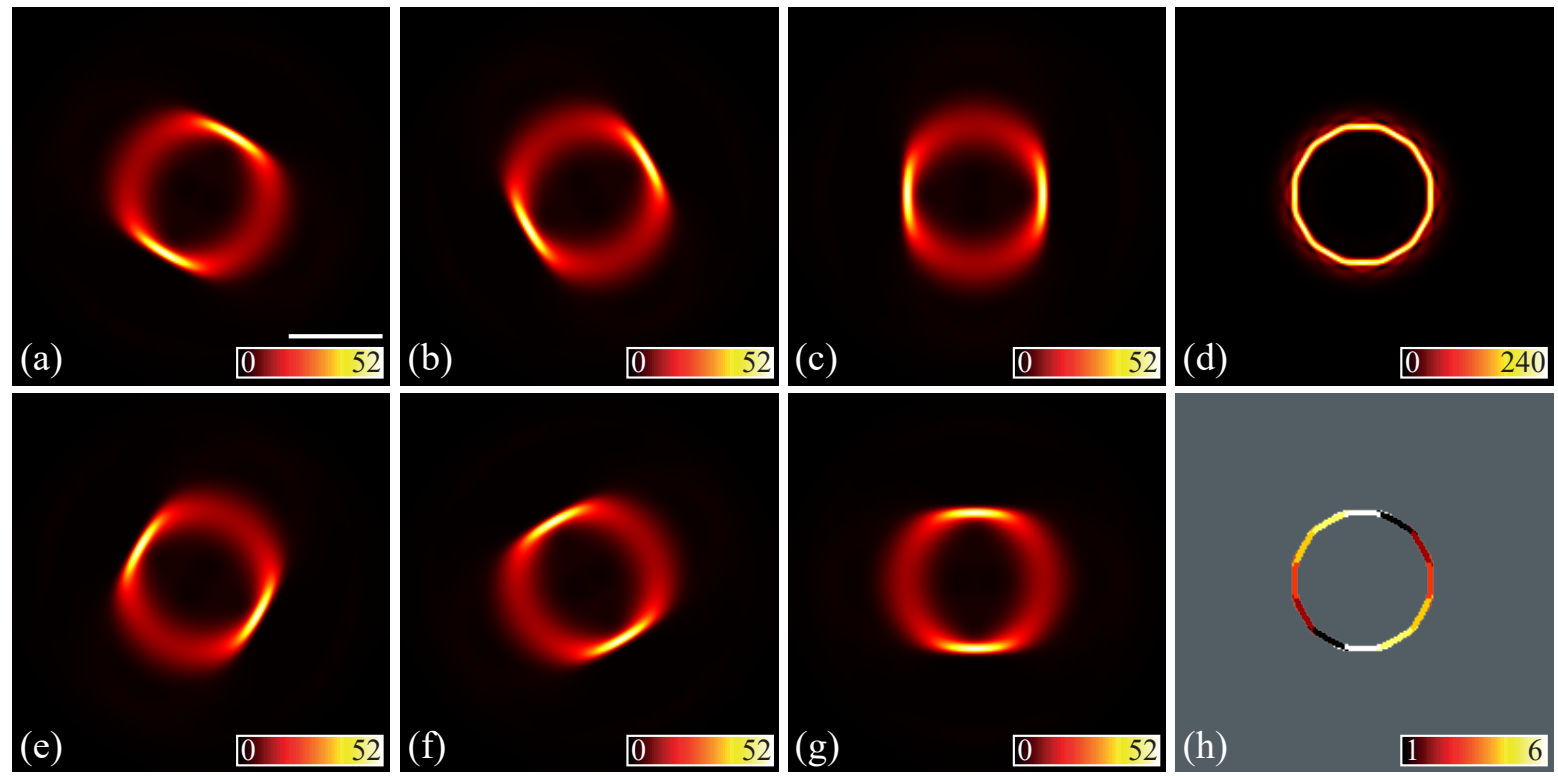

Figure 4.14: Analysis of suitable criteria for determination of a filament's position and direction. (a-c,e-g) For a pixel size of $10 \mathrm{~nm}$, the resulting image of a circular structure with a width of two pixels is calculated for six equally spaced $1 \mathrm{D}$ depletion pattern orientations for a resolution enhancement of $k=8$. The scale bar indicates a length of $500 \mathrm{~nm}$. (d) For each pixel, the variance of the intensity over the pattern orientations is calculated and displayed. (h) Analogously, the pattern direction yielding the maximum signal count is depicted for each pixel, with 1 denoting the direction yielding an angle of $60^{\circ}$ with the $y$-axis (as in (a)) and 6 denoting the orientation enclosing an angle of $-90^{\circ}$ with the $y$-axis (as in $(\mathrm{g})$ ). Note that a mask (gray) defined by the variance (d) is applied to the data. 
tions can be taken into account for the decision algorithm. Moreover, no complex analysis or reconstruction algorithms can be employed due to limited processing power on the FPGA. Furthermore, for being a valuable tool for live-cell imaging of moving structures, the decision has to be taken in the order of the pixel dwell time and the algorithm needs to be robust against possible fluctuations in the total signal count.

For determining the scan pattern, both the structure's position and its orientation need to be identified on a pixel basis, i.e. taking into consideration only the signal acquired on a single pixel for different depletion pattern orientations. For recognizing the structure's position, the dependence of the signal's variance on the depletion pattern orientation has been identified as a suitable criterion. Applying this criterion on a big field of view yields the results depicted in figure 4.14(d). On each pixel, the resulting signal count for all six pattern orientations is taken into account and the variance of those six values is calculated. Obviously, the variance is high close to the structure, while it is low in the absence thereof. For a filament tracing algorithm, this implies the necessity of scanning a line roughly perpendicular to the structure, since this yields the most pronounced peak of the variance. The maximal variance along this line then indicates the structure's position.

The direction of a filamentous structure can be determined directly by comparing the signal on a single pixel obtained for the different pattern orientations. Therefore, the orientations are numbered as 1 to 6 with $n$ denoting the orientation enclosing an angle of $90^{\circ}-n \cdot 30^{\circ}$ with the $y$-axis. The orientation yielding the maximal signal count for each pixel is displayed in figure 4.14(h). For a better visualization, the data is masked such that the resulting value is set to zero wherever the variance at that position is less than $30 \%$ of the overall maximal variance (cf. figure 4.14(d)). It is clearly evident that the chosen pattern orientation can actually well approximate the real direction of the structure under analysis.

Hence, the filament's position and direction can be uniquely determined by first identifying the structure's position, employing the signal's variance, and then determining the pattern orientation yielding the maximal signal at the position of maximal variance.

Based on these results, a tracing algorithm can now be developed. For biological samples, an upper limit on the structure's curvature can be assumed, implying that a filament's direction typically does not change drastically within the course of some pixels. Therefore, the filament's direction does not need to be determined on a line-wise basis. Instead, small blocks of lines are scanned, with the decision algorithm conducted at the end of each block, and the number of lines per block is chosen depending on the maximal curvature.

Hence, for a given starting position and direction, a scan of a pre-defined number of lines of a certain length, i.e. pixel number, is conducted (cf. also figure 3.17) on a pre-defined scanning grid. The orientation of these lines is chosen according to the direction of the structure: For a horizontally oriented structure, i.e. pattern orientation 6 (see figure $4.15(\mathrm{f})$ ), the lines are 


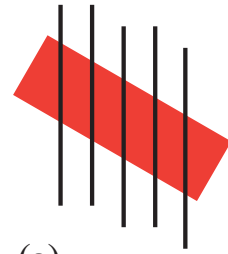

(a)

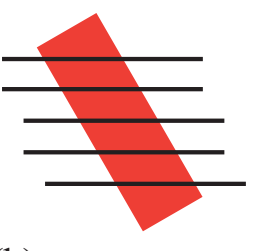

(b)

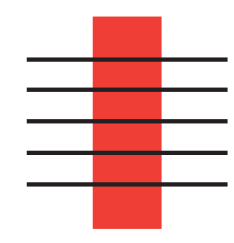

(c)

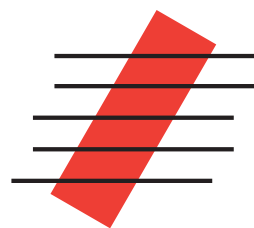

(d)

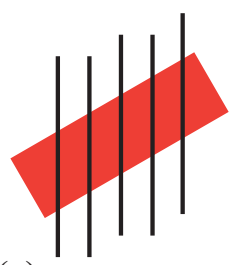

(e)

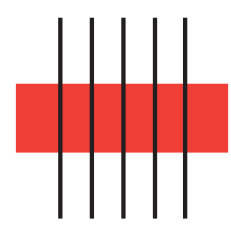

(f)

Figure 4.15: Sketch of the scan pattern for the tracing algorithm. For a structure direction corresponding to an optimal depletion pattern orientation of 1 to 6 (cf. figure 4.14(h)), the line scan for the tracing algorithm is chosen as indicated in (a-f) based on a pre-defined scanning grid. A displacement of the lines in $x$ and $y$ ensures the best possible approximation of the structure's direction.

initially chosen vertically. Each line is displaced by one pixel in $x$-direction relative to the previous line, as schematically illustrated in figure 4.15(f). Analogously, horizontal lines are chosen for a vertically oriented structure, as indicated in figure 4.15(c). For a structure orientation yielding the depletion pattern orientation 1 optimal, as shown in figure 4.15(a), the lines are not purely displaced in $x$-direction, but also in $y$-direction in order to yield an optimal scanning of the structure. Therefore, every two lines experience a shift in $y$-direction by one pixel, and analogously in $x$-direction for the pattern orientations 2 (see figure 4.15 (b)) and 4 (see figure $4.15(\mathrm{~d})$ ).

After a pre-defined number of lines has been scanned, the signal counts for the last scanned line are evaluated to determine the current position and direction of the filament. The pixel yielding the maximal variance between pattern orientations is identified as the new position of the structure. For that pixel, the pattern orientation resulting in the highest signal count is determined and identified as the new structure's direction. The scan pattern for the next block can now be fully determined according to figure 4.15 and the scan is continued.

The tracing algorithm is first tested in MATLAB on a simulated filamentous structure. A resolution enhancement of $k=8$ is chosen for the simulated PSFs, necessitating at least 6 pattern orientations when employing Richardson-Lucy deconvolution. The corresponding simulated images are displayed in figure 4.16 (a-c,e-g). The data is reconstructed by both maximum-value reconstruction (see figure 4.16(d)) and Richardson-Lucy deconvolution (see figure 4.16(h)), with the former showing slight artifacts at the turns of the filaments due to the insufficient sampling with pattern orientations. Figure 4.16 (i-k,m-o) show the images acquired by the tracing algorithm for the six pattern orientations. Clearly, the inner ring of the structure is successfully detected and the scanning is limited to a band which fully includes the filament. As seen in comparison to e.g. figure 4.16(h), also turns of the structure are correctly recognized. Note that the algorithm is stopped before the starting position is reached again. The data can be reconstructed employing either maximum-value reconstruction or Richardson-Lucy deconvolution, with the results presented in figure 4.16(1) and (p), respectively. Both algorithms yield a good estimation of the structure as compared to figure 4.16(d) and (h). Nonetheless, at the 


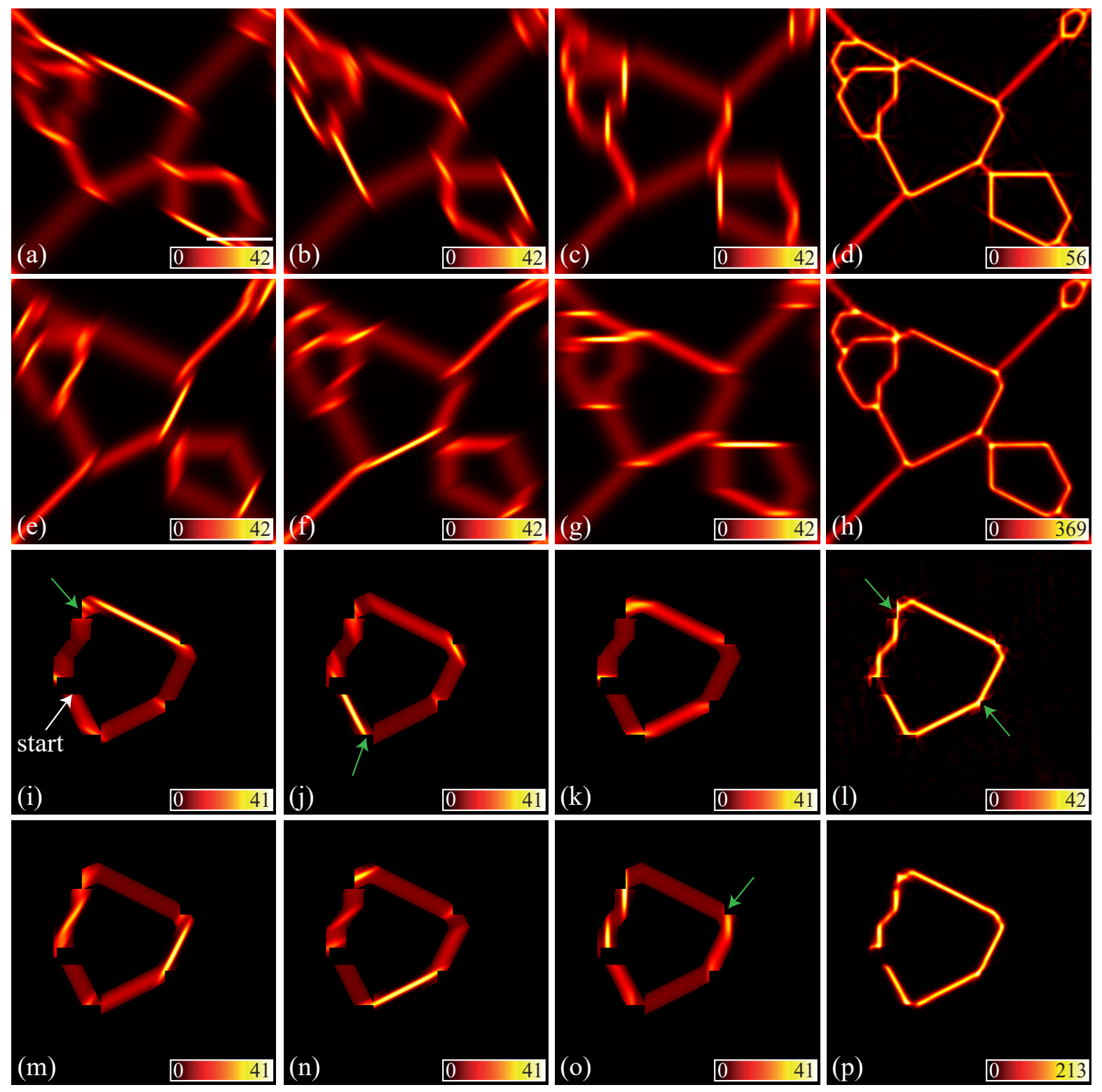

Figure 4.16: Results of the first tracing algorithm on a simulated filamentous structure. A filamentous structure is simulated for a pixel size of $5 \mathrm{~nm}$ with a structure width of three pixels. (a-c,e-g) Resulting images of the simulated structure for six equally spaced 1D depletion patterns for a resolution enhancement of $k=8$. The scale bar indicates a length of $500 \mathrm{~nm}$. (d) Maximum-value reconstruction of the data shown in (a-c,e-g). Negative pixel, resulting from discontinuities in the assembled OTF (cf. [Krü17]), are set to 0 for the visualization. (h) Richardson-Lucy deconvolution of the same data with 10 iteration steps and $\alpha_{R c h}=0.0001$. (i-k,m-o) Images acquired by the tracing algorithm for the six depletion pattern orientations. Only a narrow band of 31 pixel, corresponding to $155 \mathrm{~nm}$, around the filament is scanned. Scan position and direction are adapted every 5 lines and 120 steps are performed in total. (l) Maximum-value reconstruction of the data (i-k,m-o). Artifacts arise due to black corners in the scan at the turns of the filament (highlighted by green arrows). (p) Richardson-Lucy deconvolution (10 iteration steps, $\alpha_{R c h}=0.0001$ ) for the same data. Also here, the filaments seem incomplete at the positions of the turns. 
turns of the filaments, black areas are visible where the filaments are incomplete (indicated by green arrows in figure 4.16), especially in the maximum-value reconstruction. These areas are already visible in the raw data and result from an incomplete scanning of the filament at the turn: If the orientation of the lines for the scan is changed from horizontal to vertical or vice versa, parts of the filament are not scanned by this version of the algorithm. This can be avoided as outlined in the following.

To optimize the algorithm, the change of a horizontal to a vertical line scan is detected during the decision process. Whenever this occurs, the scan is not continued with the newly determined scan position and direction, but instead it is still continued in the same direction as before for a specified number of additional lines. Nevertheless, the newly determined parameters are saved and employed as starting position in the next iteration step. The results of this improved tracing algorithm on simulated data are shown in figure 4.17(a-c,e-g) for the six depletion pattern orientations. The additional line scans at turns of the filament are clearly visible and result in a more complete reconstruction, as seen from figure $4.17(\mathrm{~d})$ and $(\mathrm{h})$ for the maximum-value reconstruction and the Richardson-Lucy deconvolution, respectively. Both reconstruction techniques yield a good approximation of the structure. At the intersection points, the maximum-value reconstruction even allows an approximation of the initial direction of the filaments outside the scanned region.
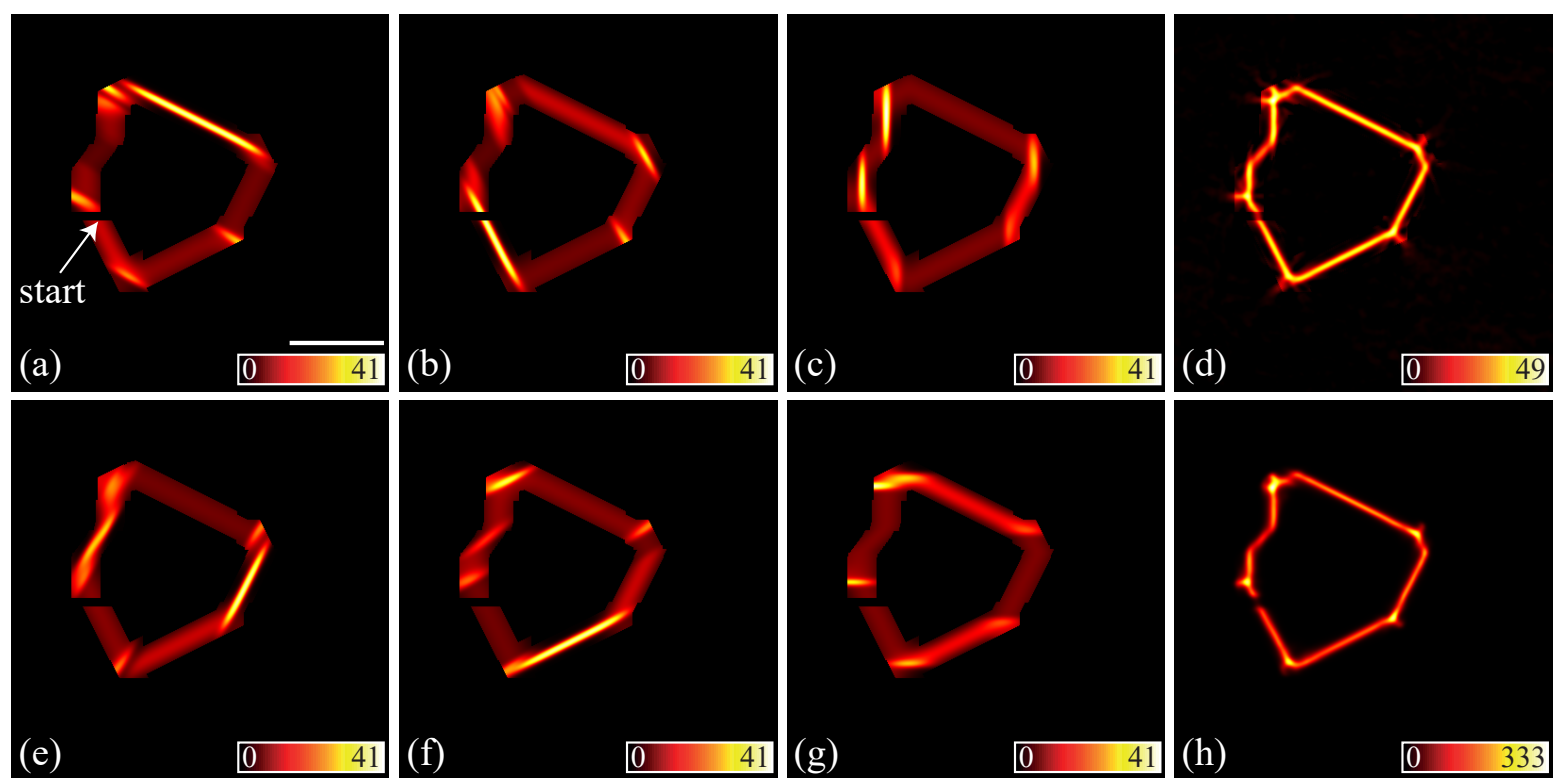

Figure 4.17: Results of the optimized tracing algorithm on a simulated filamentous structure. The same structure as in figure 4.16 is simulated. The tracing algorithm is optimized to avoid the formation of left-out corners in the scan by increasing the number of lines before turns. The results for the six depletion pattern orientations for otherwise same parameters as in figure 4.16 are displayed in (a-c,e-g). (d) shows the maximum-value reconstruction and (h) the Richardson-Lucy deconvolution (10 iteration steps, $\left.\alpha_{R c h}=0.0001\right)$ for the data shown in (a-c,e-g). 


\subsubsection{Tracing of actin filaments}

The tracing simulation, as described beforehand, is subsequently translated into a self-written LabVIEW routine, as already highlighted in section 3.5 (cf. figure 3.17). The results obtained by utilizing this LabVIEW routine for the tracing of actin filaments in vitro as well as of microtubules in fixed and living fibroblasts are here reported. The measurements are performed with six different depletion pattern orientations and reconstruction results for both maximum-value and Richardson-Lucy deconvolution are presented.

Before the tracing algorithm is employed, a confocal scan is performed with the Imspector software. Starting position and direction as well as the field of view as underlying grid for the acquisition are determined from that Imspector image and handed over to the LabVIEW routine. The tracing is subsequently performed on the selected filament for a pre-defined number of steps.

An exemplary measurement on actin filaments is depicted in figure 4.18. Figure 4.18(a) shows the confocal scan, highlighting the starting point chosen for the tracing as well as the rough course of the chosen filament. The output from the tracing algorithm is displayed in figure 4.18(b), yielding the raw data for the six different pattern orientations. The gray area indicates non-scanned regions of the overall field of view, visualizing clearly the thin band around the filament which is scanned by the algorithm. Figure 4.18(c) shows the maximumvalue reconstruction of the data in figure 4.18 (b), whereas in figure 4.18 (d) the results of the Richardson-Lucy deconvolution are depicted. Occasionally, the scan region does not fully cover the filament, as e.g. indicated by the green arrow in figure 4.18(c). This might partly be due to the relatively low number of pixels per line, which are chosen as 15 here, but, since it is also more prone to happen at crossings of the filaments, it will be subject to future investigations
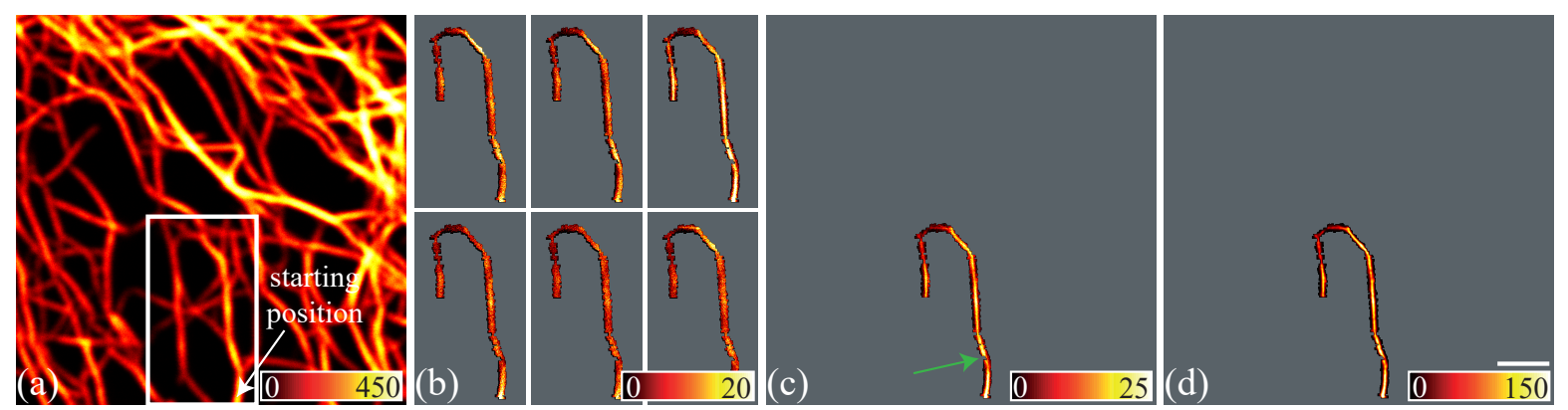

Figure 4.18: Tracing of actin filaments in vitro: (a) The confocal scan, performed with Imspector, enables the choice of a starting point and direction and is used to set the underlying grid for the tracing algorithm. The acquisition is performed with $P_{\text {exc }}=1.19 \mu \mathrm{W}$, a pixel size of $20 \mathrm{~nm}$ and pixel dwell time of $30 \mu \mathrm{s}$. (b) The raw data for the six pattern orientations are the output from the tracing algorithm for $P_{\text {exc }}=2.6 \mu \mathrm{W}, P_{\mathrm{STED}}=48 \mathrm{~mW}$, a pixel size of $20 \mathrm{~nm}$ and a pixel dwell time of $8 \mu$ s per pattern direction. The tracing is performed for 15 pixels per line for blocks of 5 lines each. (c) Maximum-value reconstruction of the data presented in (b) and (d) Richardson-Lucy deconvolution of the same data with 10 iteration steps and $\alpha_{R c h}=0.0001$. The scale bar is valid for all images and indicates a length of $2 \mu \mathrm{m}$ and the gray area indicates non-scanned regions of the overall field of view. 
and improvements of the algorithm as outlined in chapter 5 .

The results presented here demonstrate the ability of the tracing algorithm to successfully identify and follow the contorted and variegated course of the actin filaments. Even filament regions characterized by a dimmer staining, as seen in the upper left turn of the filament, do not cause disruptions, enabling a filament tracing for several $\mu \mathrm{m}$. For a comparison with the tomoSTED recordings presented in subsection 4.1.2, filament tracing is performed on microtubules in fixed cells, as demonstrated in the following subsection.

\subsubsection{Tracing of microtubules in fixed fibroblasts}

Actin filaments in vitro, as presented in the previous subsection, are a filamentous structure, but do not come with the typical challenges of cell imaging: The density of the structure can be adapted, since it is governed by the chosen dilution during preparation, and the labeling background is negligible. This is caused by the absence of other structures as well as the restriction of the actin filaments to a thin layer only. Also the properties of the SiR-actin dye are beneficial
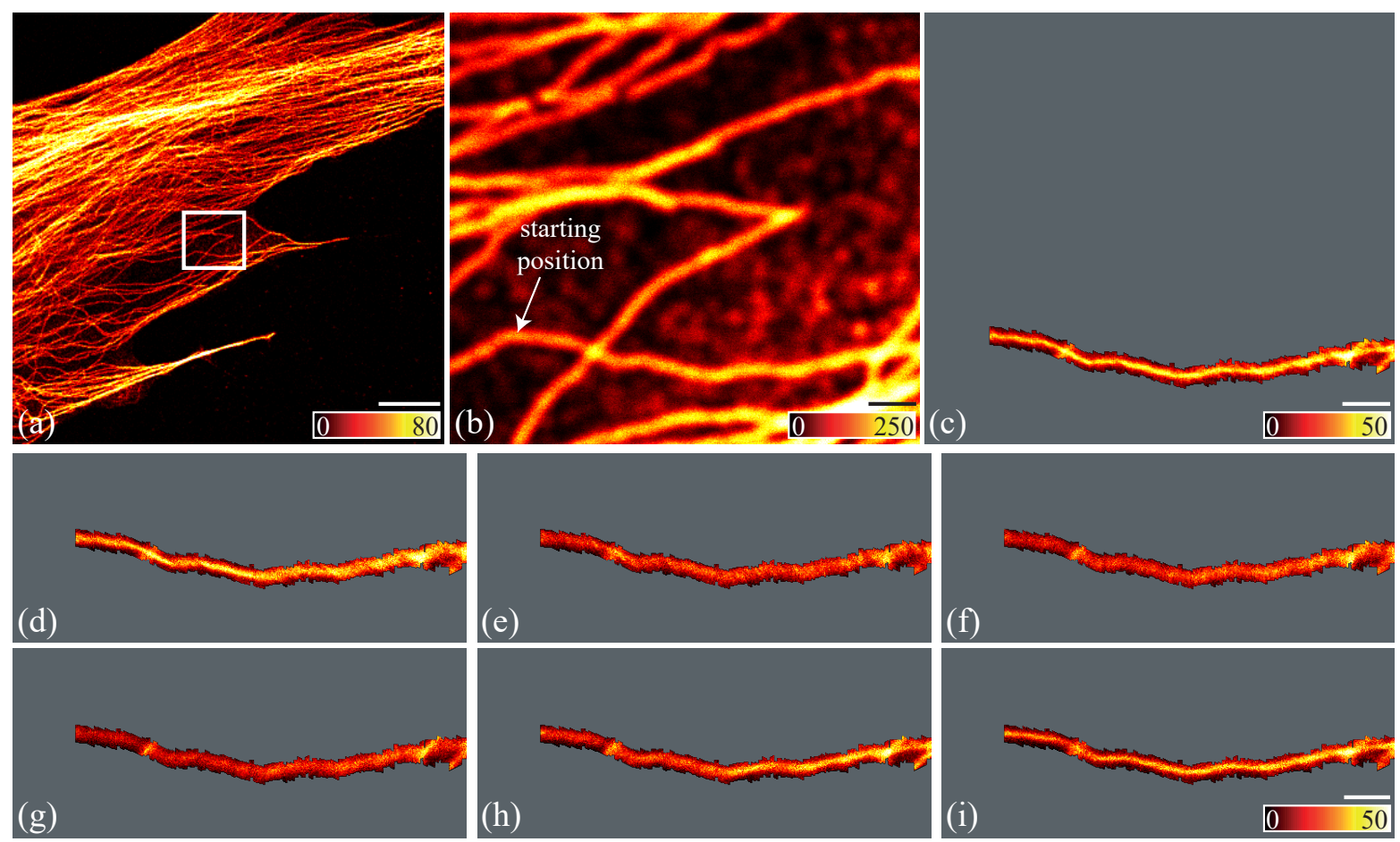

Figure 4.19: Tracing of $\alpha$-tubulin in fixed fibroblasts (dye: Abberior STAR 635P): (a) In the confocal overview, acquired with $P_{\mathrm{exc}}=1.19 \mu \mathrm{W}$, a pixel size of $200 \mathrm{~nm}$ and pixel dwell time of $10 \mu \mathrm{s}$, the area chosen for the closeup in (b), imaged with $P_{\text {exc }}=3.4 \mu \mathrm{W}$, a pixel size of $20 \mathrm{~nm}$ and pixel dwell time of $30 \mu \mathrm{s}$, is indicated. The starting position and direction for the filament tracing are indicated in (b), with the results depicted in $(\mathrm{d}-\mathrm{i})$. The acquisition parameters are chosen as $P_{\mathrm{exc}}=10.7 \mu \mathrm{W}, P_{\mathrm{STED}}=35 \mathrm{~mW}$, a pixel size of $20 \mathrm{~nm}$ and a pixel dwell time of $10 \mu$ s per pattern direction. The tracing is performed for 19 pixels per line for blocks of 5 lines each. (c) Maximum-value reconstruction of the raw data in (d-i). The scale bar indicates a length of $10 \mu \mathrm{m}$ in (a) and $1 \mu \mathrm{m}$ in all other images. 
in this case: It specifically binds to F-actin and increases its fluorescence intensity more than 100-fold [LRD $\left.{ }^{+} 14\right]$ upon binding, yielding a highly specific staining with low labeling background. Especially the low background signal obviously facilitates imaging and in particular filament tracing which relies on detecting differences in signal. Nevertheless, the same tracing algorithm is shown to successfully trace filaments also in the challenging conditions offered by the cell cytoplasm environment.

Figure 4.19(a) shows a confocal overview of microtubules stained in fixed fibroblasts. The area chosen for the closeup in figure 4.19(b) as well as for the underlying grid for the tracing algorithm is highlighted. From the chosen starting position, the tracing is performed over several $\mu \mathrm{m}$, with the results displayed in figure 4.19 (d-i) for the six different depletion pattern orientations. The corresponding maximum-value reconstruction is shown in figure 4.19(c). The band scanned around the structure of interest is again clearly visible, and the algorithm correctly identifies the course of the filament despite the noticeable background (cf. figure 4.19(b)), proving its suitability for the imaging of biological structures.

As has been shown so far, the employed algorithm correctly traces the desired structure, but no conclusion about the quality of the resulting image or the obtainable resolution was drawn. For this, the results of the tracing algorithm are compared to a conventional tomoSTED acquisition, as presented in subsection 4.1.2. Figure 4.20 depicts such a comparison of both acquisition modes. In figure 4.20(a), a confocal scan is displayed, highlighting a line profile employed for comparing the resolution between the reconstructed tomoSTED images (b) and (c) and the reconstructed tracing images (e) and (f) employing maximum-value reconstruction and Richardson-Lucy deconvolution, respectively. The profile plot in figure 4.20(d), obtained by averaging over five neighboring lines, yields the same observed structure size in the maximum-value reconstruction for both the tomoSTED acquisition and the filament tracing. The comparison of the resulting reconstructed images does not present any sign of artifacts or distortions caused by the selective scanning performed during the tracing routine.

Hence, this tracing method successfully yields not only a localization of the desired structure, but also enables super-resolution imaging without the need of scanning the whole field of view.

Two further examples of the tracing algorithm are displayed in figure 4.21(a-d) and (e-h). In figure 4.21(a) and (e), a confocal overview of the cell is shown, indicating the areas for the closeups in (b) and (f), respectively, with the starting positions for the tracing algorithm highlighted therein. Both maximum-value reconstruction (cf. figure 4.21 (c,g)) and Richardson-Lucy deconvolution (cf. figure $4.21(\mathrm{~d}, \mathrm{~h})$ ) of the raw data are displayed. In figure 4.21(c,d), the tracing algorithm is performed twice for two different starting positions within the same field of view, demonstrating the ability of iteratively scanning all structures of interest within a certain area. However, a more elaborate decision algorithm needs to be employed at positions of crossing filaments as discussed in chapter 5 in order to avoid multiple scanning of the same filament. 

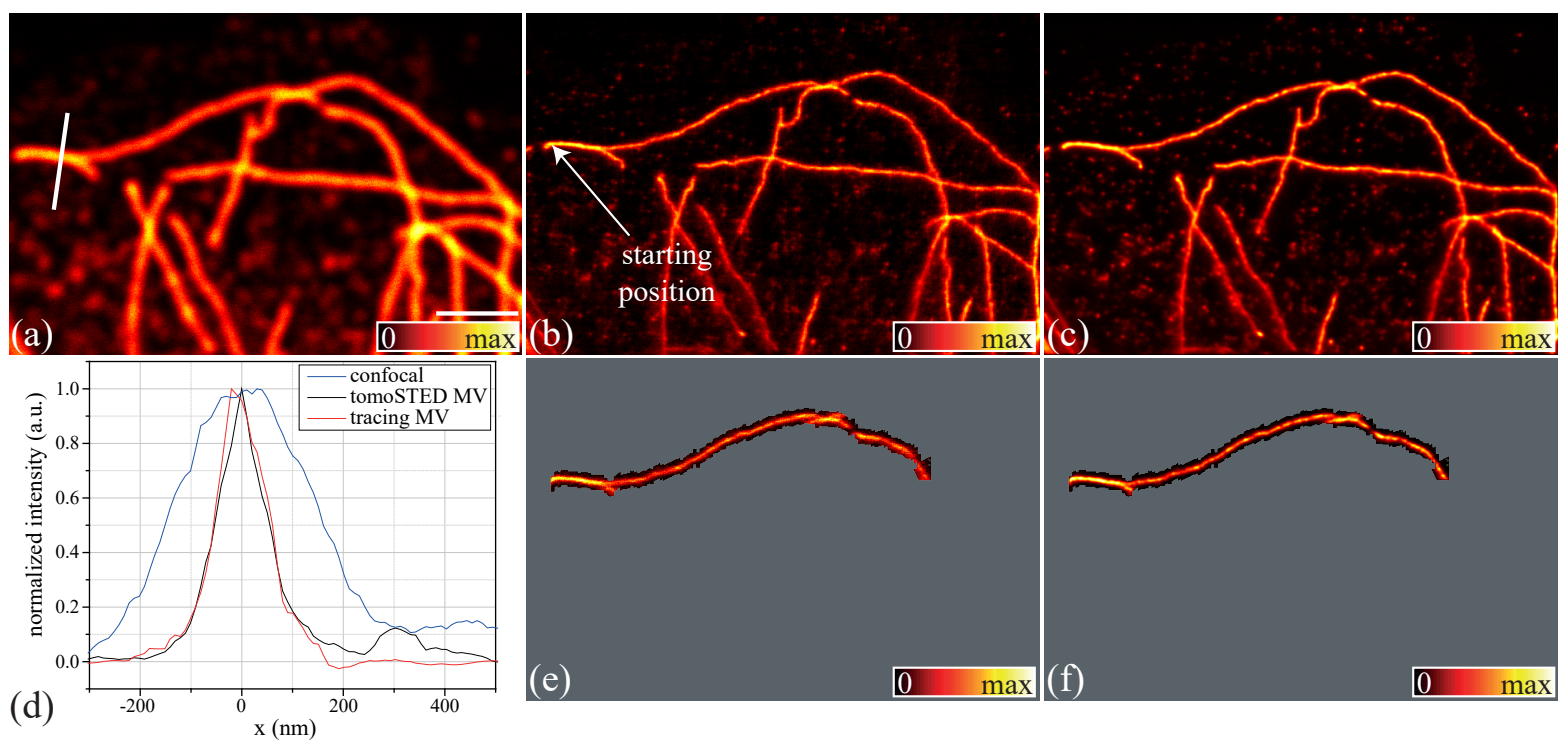

Figure 4.20: Comparison between tomoSTED acquisition and filament tracing. (a) Confocal scan of $\alpha$-tubulin stained in fixed fibroblasts (dye: Abberior STAR 635P) acquired with $P_{\text {exc }}=2.0 \mu \mathrm{W}$, a pixel size of $20 \mathrm{~nm}$ and pixel dwell time of $30 \mu \mathrm{s}$. The scale bar indicates a length of $2 \mu \mathrm{m}$. (b) Maximumvalue reconstruction and (c) Richardson-Lucy deconvolution (10 iteration steps, $\alpha_{R c h}=0.0001$ ) of a standard tomoSTED acquisition with pixel-wise switching of six pattern orientations, acquired with $P_{\text {exc }}=3.4 \mu \mathrm{W}, P_{\mathrm{STED}}=48 \mathrm{~mW}$, a pixel size of $20 \mathrm{~nm}$ and pixel dwell time of $10 \mu \mathrm{s}$ per direction. The same acquisition parameters are chosen for the filament tracing with the starting position indicated in (b). The tracing is performed for 19 pixels per line for blocks of 5 lines each. The maximum-value reconstruction and Richardson-Lucy deconvolution of the data acquired by the tracing algorithm are displayed in (e) and (f), respectively. Line profiles (averaged over five neighboring lines) of a filament, as indicated in (a), drawn for the confocal scan as well as the maximum-value reconstruction of both the standard tomoSTED acquisition and the tracing result are shown in (d).

Figure 4.21(c,d) moreover demonstrates that the algorithm can also handle short disruptions of the filament at the image border, simply continuing the scan hereafter.

The second example, depicted in figure $4.21(\mathrm{~g}, \mathrm{~h})$, shows two further characteristics of the algorithm. First, the algorithm is designed to follow a single filament, meaning that the number of pixels per line is chosen to cover the width of a single filament. For two filaments next to each other, as identified by the STED acquisition in figure $4.21(\mathrm{~g}, \mathrm{~h})$ and not immediately apparent from the confocal scan in figure 4.21 (f), the scanned lines will be centered around one of the two filaments. This might leave the other one only partly scanned, as seen in the right part of figure $4.21(\mathrm{~g}, \mathrm{~h})$. Further adaptations of the algorithm, as outlined in chapter 5 , will resolve this issue, leading to a full scan of both filaments. The second characteristic is immediately visible considering the ending filament in the left part of figure $4.21(\mathrm{~g}, \mathrm{~h})$. Since no signal is detected in the continuation of this filament, the algorithm will gradually yield a turn of the direction, causing a re-scan of the same filament in reverse. After returning to the crossing point, the tracing can continue along any other filament. In this way, also ending filaments can be correctly detected and scanned. 


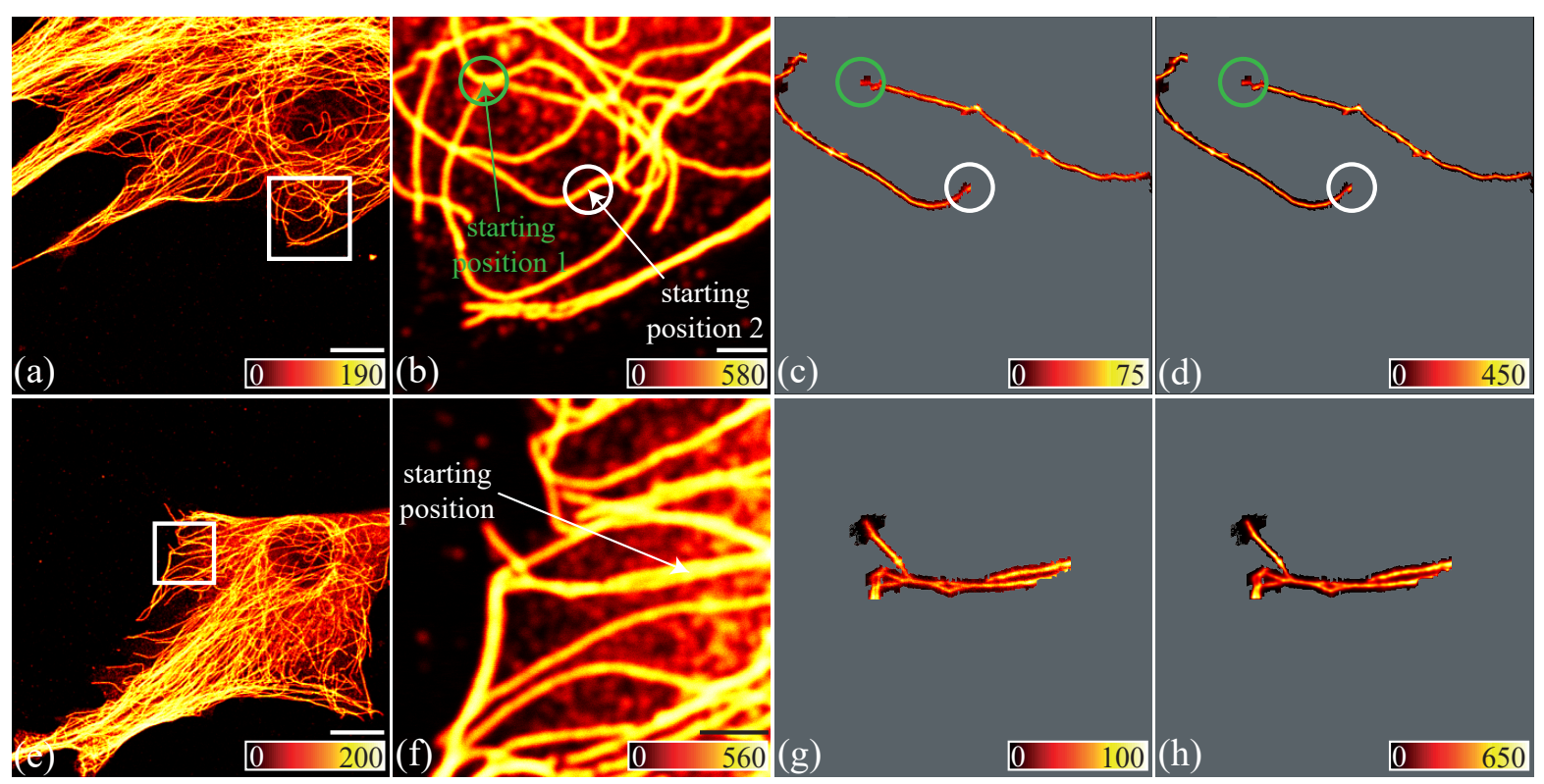

Figure 4.21: Tracing of microtubules in fixed fibroblasts (dye: Abberior STAR 635P). (a) Confocal overview, acquired with $P_{\text {exc }}=1.19 \mu \mathrm{W}$, a pixel size of $200 \mathrm{~nm}$ and pixel dwell time of $10 \mu \mathrm{s}$, and (b) confocal closeup of the highlighted area, imaged with $P_{\text {exc }}=3.4 \mu \mathrm{W}$, a pixel size of $20 \mathrm{~nm}$ and pixel dwell time of $30 \mu \mathrm{s}$. The tracing algorithm is performed twice on this field of view for different starting positions, indicated in (b). The tracing is performed for 15 pixels per line for blocks of 7 lines each (starting position 1) and 19 pixels per line for blocks of 5 lines each (starting position 2), respectively. The corresponding results are displayed via the (c) maximum-value reconstruction and the (d) Richardson-Lucy deconvolution (10 iteration steps, $\left.\alpha_{R c h}=0.0001\right)$. The acquisition parameters are set as $P_{\text {exc }}=3.2 \mu \mathrm{W}, P_{\mathrm{STED}}=48 \mathrm{~mW}$, a pixel size of $20 \mathrm{~nm}$ and pixel dwell time of $10 \mu$ s per direction. (e) Confocal overview of a different cell, acquired with the same parameters as for (a), and (f) confocal closeup of the highlighted area, imaged with the same acquisition parameters as chosen for (b). Tracing is performed for the starting position indicated in (b) for 19 pixels per line for blocks of 5 lines each, with the (g) maximum-value reconstruction and (h) Richardson-Lucy deconvolution of the raw data. The acquisition parameters are set as $P_{\text {exc }}=7.9 \mu \mathrm{W}, P_{\mathrm{STED}}=48 \mathrm{~mW}$, a pixel size of $20 \mathrm{~nm}$ and pixel dwell time of $10 \mu \mathrm{s}$ per direction. The scale bar in (a,e) is set to $10 \mu \mathrm{m}$, whereas in all other images it indicates a length of $2 \mu \mathrm{m}$.

\subsubsection{Tracing of microtubules in living fibroblasts}

In living cells, the filaments' movement can cause motion artifacts for a typical image scan with predefined area, since this area is usually much larger than strictly necessary in order to ensure that the structure of investigation is fully enclosed. Hence, imaging by means of a tracing algorithm can allow to observe dynamics which are otherwise not accessible, because only the structure of interest is imaged. Moreover, in this way areas of the sample which are not of interest for the process under investigation are not exposed to light. The imaging of living cells is particularly demanding due to their non-deterministic movement as well as the requirement for a live-cell compatible staining procedure. Additionally, phototoxicity and sample damage may result, implying that not every measurement protocol employed for fixed cells can be directly transferred to live-cell imaging. 
As the application of the tracing algorithm to the imaging of living cells is especially appealing, this subsection is dedicated to show the performance of this imaging scheme under these, compared to fixed cells more challenging, imaging conditions.

Microtubules are stained in living fibroblasts as described in the appendix B and subsequently imaged within the first hour after staining. Figure 4.22(a) exemplarily shows a confocal overview of a cell. The highlighted subset is subsequently chosen for a more detailed analysis. For this subset, a confocal pre-scan is performed, as shown in figure 4.22(b) with the red color table. Within the subsequent 5 minutes, the tracing algorithm is employed on that sample region for two different starting points, with the results shown in figure 4.22 (c) in blue. Here, the final image is reconstructed from the raw data via the Richardson-Lucy deconvolution and for better visibility, the color scale for the tracing results is enhanced. In red, the confocal pre-scan is shown again for comparison, and a further confocal scan, shown in green in (b) and (c), is performed immediately after the two tracings. The filament's movement is clearly visible in both subsets, indicating a displacement and change of shape between the first confocal, the respective tracing and the second confocal scan. The filament can be clearly identified to be the same in all three scans, implying that the algorithm detects each filament's current position and direction. A line scan, shown in figure 4.22(d) and indicated in (c), highlights the movement as well as the resolution enhancement of the tracing result compared to the confocal scans.
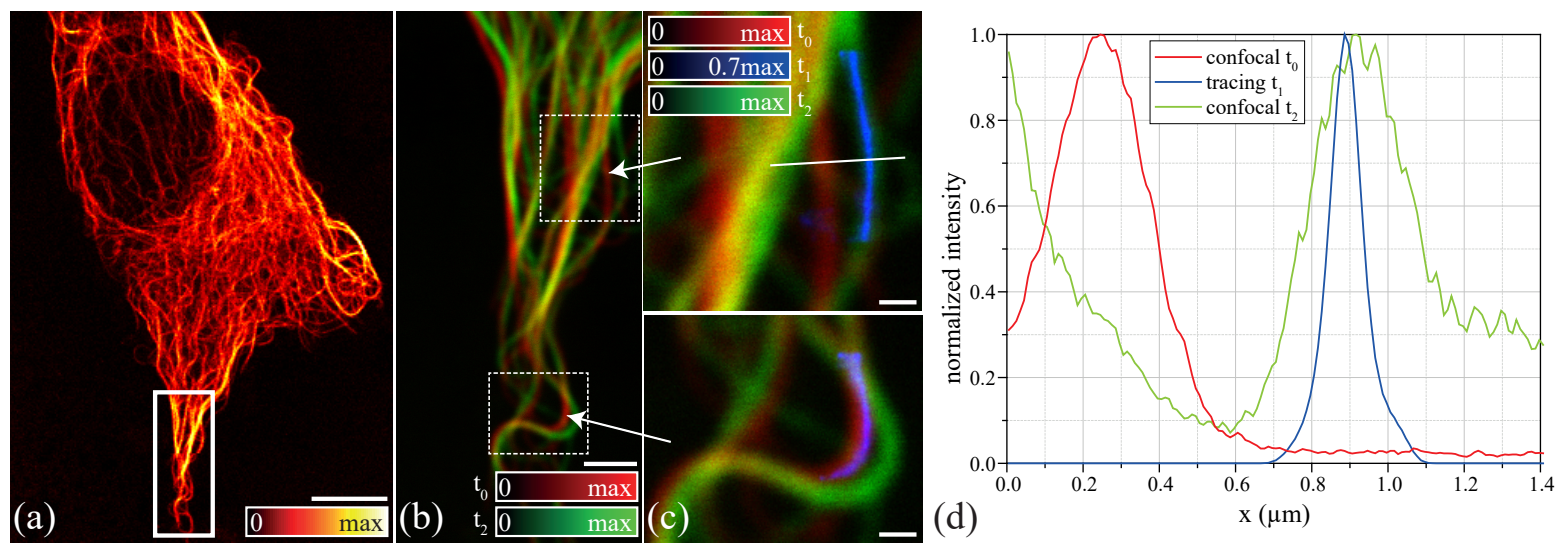

Figure 4.22: Tracing of microtubules in living fibroblasts (dye: SiR). (a) Confocal overview, acquired with $P_{\text {exc }}=6.7 \mu \mathrm{W}$, a pixel size of $200 \mathrm{~nm}$ and pixel dwell time of $10 \mu \mathrm{s}$, and (b) confocal closeup of the highlighted area at two time points $t_{0}$ and $t_{2} \approx t_{0}+5 \mathrm{~min}$, imaged with $P_{\text {exc }}=3.4 \mu \mathrm{W}$, a pixel size of $20 \mathrm{~nm}$ and pixel dwell time of $30 \mu \mathrm{s}$. The tracing algorithm with 19 pixels per line for blocks of 5 lines each is performed twice on this field of view for different starting positions, as indicated in (b). (c) The results of the tracing algorithm (blue, Richardson-Lucy deconvolution with 10 iteration steps, $\left.\alpha_{R c h}=0.0001\right)$ are displayed as overlay with the two confocal images. The tracing was performed at a time point $t_{1}$ with $t_{0}<t_{1}<t_{2}$. The acquisition parameters for the tracing are set as $P_{\mathrm{exc}}=10.6 \mu \mathrm{W}$, $P_{\text {STED }}=40 \mathrm{~mW}$, a pixel size of $20 \mathrm{~nm}$ and pixel dwell time of $10 \mu$ s per direction. The scale bar in (a) is set to $10 \mu \mathrm{m}$, whereas in (b) it indicates a length of $2 \mu \mathrm{m}$. In (c) it depicts $500 \mathrm{~nm}$. (d) shows a line profile, averaged over 10 neighboring lines, as indicated in (c). The filament's movement as well as the resolution enhancement of the tracing compared to the confocal scans is clearly visible. 
Hence, the algorithm is able to identify and trace moving filaments in living cells with superresolution and is thus generally compatible with live-cell imaging. This paves the way for many biological applications, which are discussed in view of further improvements to the algorithm in chapter 5 . 


\section{Conclusion and Outlook}

In the here presented thesis, adaptive scanning for STED microscopy with the focus on lowillumination and fast-scanning imaging schemes has been presented and successfully applied to a variety of biological samples.

TomoSTED microscopy, as introduced in section 2.2. has been shown in the past to yield images of uncompromised quality at a reduced light dose and acquisition time for a line-wise switching of the pattern orientation when compared to the classical 2D STED variant. It can directly be combined with other sample-conserving techniques like RESCue and is therefore the STED variant of choice when low-illumination imaging is required. Unfortunately, with a line-wise switching tomoSTED microscopy cannot provide the same flexibility as the classical 2D STED variant, since the same pixel needs to be scanned repetitively with a time offset of more than several hundred $\mu \mathrm{s}$.

Thus, a method to generate 1D depletion patterns with the possibility for pixel-wise switching has been developed within this work by employing the phenomenon of conical diffraction (cf. subsection 2.4.3). By extensive simulations, as presented in section 3.2, optical design parameters for a suitable focal intensity distribution have been identified. It has been shown that utilizing this intensity distribution as a 1D depletion pattern provides the same performance as the previously employed pattern, which was generated by phase-modulation. Using conical diffraction for pattern generation has the advantage that the pattern orientation can be controlled by polarization optics, which is why a special focus is put on the generation of suitable polarization states (cf. section 3.3 by utilizing Pockels cells. The experimental implementation, as described in section 3.1 has shown to yield satisfactory results: The depletion pattern compares well with the standard 1D depletion pattern, as seen from resolution measurements on fluorescent microspheres (cf. subsection 4.1.1). Not only the single depletion pattern exhibits the same pattern steepness as the standard 1D depletion pattern, which is the crucial parameter for the resolution enhancement, but also the recording of tomoSTED images with a pixel-wise switching of the depletion pattern orientation yields the expected high resolution in the final reconstructed image. By acquiring super-resolved images of microtubules in fixed Vero cells (as shown in subsection 4.1.2, tomoSTED microscopy with the novel pixel-wise switched depletion pattern has definitely proven its strength and its feasibility whenever it comes to imaging biological samples with a combination of high resolution, fast acquisition and light-dose reduction.

To further improve the performance of this novel tomoSTED microscopy variant with respect to light dose saving and acquisition speed, innovative adaptive scanning methods come into play. The here presented methods are based on the simple but efficient idea of determining the next step of a non-predefined scan pattern by the actual detected fluorescence signal, enabled by employing a fast-responding scanning device. A scan system with a large field of 
view and a response time below the typical pixel dwell time is not available yet on the market. After investigating the state-of-art scanning possibilities already available in the life- and material sciences, a combination of a galvanometer scanner with electro-optic deflectors has been identified as the most suitable candidate. The detailed characterization of this scan system's components as well as of its self-written LabVIEW control software has been outlined in section 3.5. The advantages derived by a careful combination of this scan system with the beforehand presented depletion pattern generation yields an innovative experimental setup (cf. section 3.1 with a high flexibility regarding the scan pattern and the switching of the depletion pattern's orientation, enabling the further investigation of high-resolution, sample-preserving and fast imaging techniques.

The first application of this innovative experimental setup has been the combination of two sample-conserving imaging techniques, namely tomoSTED microscopy and RESCue. RESCue is widely appreciated for its ability of reducing the light dose, but does not provide any improvement of the acquisition speed, which is highly desirable for modern biological applications. The here presented RESCue variant, namely FastRESCue, exploits the features of the here presented novel adaptive scanning technique in order to translate the light dose saving into an acquisition time saving: While in RESCue the lasers are simply switched off for the remaining pixel dwell time when certain signal conditions are fulfilled, in FastRESCue the lasers are left on, but the scanning is immediately advanced to the following pixel. The utilized LabVIEW routine for the setup's control (described in section 3.5 has shown its suitability for imaging as reported in subsection 4.2.1. Comparing standard image scans performed via the previously employed Imspector software and via this LabVIEW routine has shown no significant differences regarding image brightness or resolution. However, when applying FastRESCue, the novel scan system has proven inevitable for an undistorted image acquisition (cf. subsection 4.2.1). In particular for FastRESCue acquisition, the scan system provides the required response times, enabling imaging at uncompromised quality when compared to the standard scan without RESCue or with classical RESCue acquisition.

To further prove the abilities of FastRESCue and confirm that the final image quality is comparable with the previously presented results for RESCue [SER ${ }^{+11}$ ], FastRESCue is subsequently applied for a 2D STED depletion pattern on various samples. Imaging of fluorescent microspheres has demonstrated the direct translation of the light dose saving into a saving of acquisition time (cf. subsection 4.2.2), enabling a reduction of both light dose and acquisition time by a factor of more than 5 compared to a non-adaptive scan. By a local image analysis of a typical filamentous sample, even an improvement of a factor of 10 can be achieved locally. Various biological samples have been studied subsequently, demonstrating a reduction of light dose and acquisition time at uncompromised image quality.

Combining FastRESCue and tomoSTED is intriguing, since their individual advantages are 
retained and the resulting reduction of light dose as well as acquisition time is potentially more than one order of magnitude. The general compatibility of both techniques has been demonstrated in subsection 4.2.2, with FastRESCue providing an individual pixel-based decision for each pattern orientation. Unfortunately, the actual technological limitations regarding the speed of the high-voltage amplifiers used to drive the Pockels cells forced the introduction of a waiting time between individual pattern orientations. Even though, the resulting switching frequency is still considerably higher than for the original tomoSTED implementation employing a spatial-light modulator, leaving also space for future improvement. A combination of FastRESCue and tomoSTED microscopy is thus, in its current technical implementation, only competitive for a line-wise or image-wise switching of the pattern orientation.

Adaptive scanning has been demonstrated to increase the acquisition speed of a RESCue acquisition by a factor of 5 by inter alia reducing the pixel time where no structure is detected. However, the time spent on such areas cannot be arbitrarily reduced due to the statistical nature of photon counting. A scanning method which can completely avoid scanning structure-free regions can hence yield an additional, considerable improvement of the acquisition speed. Such a scan pattern has been developed within this work, with a successful application to filament tracing (cf. section 4.3), where only a small band around the filaments is scanned. By exploiting the characteristics of the depletion patterns employed for tomoSTED microscopy, the determination of the scan direction can be taken on-line without any need for further assumptions or even for information based on previously scanned positions: Imaging a filamentous structure with a 1D effective PSF, whose orientation matches with the structure's direction, yields the highest signal count. The actual structure's direction can thus be determined by rotating the PSF's orientation (cf. subsection 4.3.1). This paves the way to an adaptable scan pattern as developed in the course of subsection 4.3.1 and successfully applied on both actin filaments in vitro (cf. subsection 4.3.2) and microtubules in fixed (cf. subsection 4.3.3) and living cells (cf. subsection 4.3.4). The developed algorithm is able to correctly identify the course of a filament also in the presence of labeling background, and the scanning of a thin band around the filament allows the reconstruction of a super-resolved image thereof, which shows the same resolution enhancement as a classical tomoSTED image. Choosing multiple starting positions for the algorithm within the same field of view allows imaging not only of one particular filament (cf. subsection 4.3.3), but in future perspective will enable imaging the whole sample region by only scanning narrow bands around the existing structure. By taking into account the signal distribution over the pattern orientations in addition to the signal intensity, a detection of crossing points will also be possible, allowing a deliberate choice of the further scan direction at these points. Moreover, the corresponding coordinates could, in a further step, be used to define new starting positions.

Several additional optimization steps can be implemented to further improve the stability and performance of the tracing algorithm. The optimal algorithm will however inevitably strongly 
depend on the sample structure and the available signal-to-noise ratio. Therefore, an algorithm developed via training a neural network is very promising: With machine learning techniques, an optimal algorithm can be obtained by providing some exemplary raw data for each type of sample.

As already outlined previously, the speed of the tracing algorithm is currently mainly limited by the waiting time necessary for switching the pattern orientation. This restriction is not fundamental to the technique itself, but imposed by the employed high-voltage amplifiers' limitations. With further developments in this technology, a faster switching can be realized, allowing a powerful combination of pixel-wise switched tomoSTED microscopy with FastRESCue. The latter can be even further optimized by the same development RESCue has undergone in the latest years: A confocal pre-scan can e.g. be employed to obtain a first map of the sample, or the number of lower thresholds can be further increased by a thorough statistical analysis. Also the control of the scan system itself can be optimized: So far, the electro-optic deflectors compensate for the total offset between desired and actual scan position, meaning the compensation includes the - for a constant pixel dwell time - constant offset shown in section 3.5. Also for a varying pixel dwell time, the compensation therefore almost always acts in the same direction, and the employed range of the electro-optic deflectors is not centered in their total scan range. As an offset of the whole field of view by some micrometers is typically acceptable, a more efficient implementation could exploit their full scan range: By subtracting a constant offset, which then depends on the average pixel dwell time (cf. section 3.5), the applied compensation by the electro-optic deflectors can be centered in their available scan range and thus yield better performances for highly varying pixel dwell times. This can allow for larger scan ranges or smaller average pixel dwell times than shown within this work.

Especially for the imaging of living cells, the available signal is a limiting factor due to bleaching. The general compatibility of the tracing algorithm with live-cell imaging has been demonstrated in subsection 4.3.4. A further step can be the repetitive scanning of the same filament in order to investigate its dynamics. With the employed samples, this was not feasible due to a too low signal count for consecutive acquisitions, preventing the algorithm to take a meaningful decision about the filament's position and direction. This aspect can be tackled by further improving the staining, but mainly by optimizing the setup's design for that specific application: By the implementation of the electro-optic deflectors in the excitation, the STED and particularly the detection beam path, half of the fluorescence signal is lost due to the polarization-dependence of the electro-optic effect. Since the deflection angle induced by the electro-optic deflectors and thus also the resulting displacement is small, the de-scanning by the electro-optic deflectors can be omitted in case a confocal detection is not essential. Thus, excitation and fluorescence can be separated prior to the electro-optic deflectors, yielding an improvement in signal by a factor of 2. This however needs to be weighed against the advantages provided by the confocal detection implemented herein, i.e. mainly the $z$-sectioning capability, and the choice will 
probably depend on the desired outcome.

The here presented adaptive scanning methods are not only applicable to STED microscopy, but to any other targeted read-out technique. Moreover, the combination with well-established techniques like DyMIN or ISM can yield further improvements regarding the employed light dose. Also an extension to dual-color STED microscopy by implementing a second excitation laser for the simultaneous observation of different structures is feasible. In this way, filament tracing can also be extended to study interactions like the course of a motor-protein on a filament.

Hitherto, adaptive scanning has been considered and realized in the view of a two-dimensional imaging technique. For cellular observations, a three-dimensional imaging of sample volumes with a resolution enhancement in all spatial directions is often preferable. An extension of the fast scan system to three dimensions can be realized by exploiting e.g. an electro-optically actuated varifocal lens for selecting the $z$-position. According to the data sheet [NTT19] and related publications [YTS09, YF14, such a lens can have response times in the range of some microseconds and thus has a re-focusing speed which is comparable to the respective quantity for the electro-optic deflectors. As RESCue, and thus also FastRESCue, is typically most efficient in three-dimensional imaging due to the high amount of structure-free volume, this application is of particular interest. For combining tomoSTED microscopy with such techniques, its concept has to be extended towards a 3D high-resolution imaging technique by rotating a line- or sheet-like PSF around two angles. Such an implementation is very promising, both as a low-illumination imaging technique, but also in the view of applications beyond imaging like three-dimensional structure tracing. 



\section{References}

[Abb73] E. Abbe. Beiträge zur Theorie des Mikroskops und der mikroskopischen Wahrnehmung. Arch. Mikrosk. Anat. 9, 413 - 468 (1873).

[Abb19] Abberior GmbH. Abberior STAR 635P. https://www.abberior.com/jtl-shop/ Abberior-STAR-635P (2019). [Online; accessed 21-March-2019].

[Abd11] A. Abdolvand. Conical diffraction from a multi-crystal cascade: experimental observations. Appl. Phys. B 103(2), 281 - 283 (2011).

$\left[\mathrm{BAS}^{+} 15\right]$ F. Bergermann, L. Alber, S. J. Sahl, J. Engelhardt, and S. W. Hell. 2000-fold parallelized dual-color STED fluorescence nanoscopy. Opt. Express 23(1), 211 223 (2015).

[Ber04] M. V. Berry. Conical diffraction asymptotics: fine structure of Poggendorff rings and axial spike. J. Opt. A: Pure Appl. Opt. 6(4), 289 - 300 (2004).

[Ber10] M. V. Berry. Conical diffraction from an N-crystal cascade. J. Opt. 12(8), 075704 (2010).

[BJ07] M. V. Berry and M. R. Jeffrey. Conical diffraction: Hamilton's diabolical point at the heart of crystal optics. Prog. Opt. 50, 13 - 50 (2007).

[BJL06] M. V. Berry, M. R. Jeffrey, and J. G. Lunney. Conical diffraction: observations and theory. Proc. R. Soc. A 462(2070), 1629 - 1642 (2006).

$\left[\mathrm{BPS}^{+}\right.$06] E. Betzig, G. H. Patterson, R. Sougrat, O. W. Lindwasser, S. Olenych, J. S. Bonifacino, M. W. Davidson, J. Lippincott-Schwartz, and H. F. Hess. Imaging Intracellular Fluorescent Proteins at Nanometer Resolution. Science 313(5793), 1642 1645 (2006).

[BREH11] P. Bingen, M. Reuss, J. Engelhardt, and S. W. Hell. Parallelized STED fluorescence nanoscopy. Opt. Express 19(24), 23716 - 23726 (2011).

[Bio14] BioAxial. COnical DIffraction Microscopy (CODIM).http://www.bioaxial.com/ (2014). [Online; accessed 07-August-2015].

[BW11] M. Born and E. Wolf. Principles of Optics. Cambridge University Press, 7th edition (2011).

$\left[\mathrm{BFD}^{+} 06\right]$ M. Bossi, J. Fölling, M. Dyba, V. Westphal, and S. W. Hell. Breaking the diffraction resolution barrier in far-field microscopy by molecular optical bistability. New J. Phys. 8(11), 275 (2006). 
[BH93] P. Boulanger and M. Hayes. Bivectors and Waves in Mechanics and Optics. Chapman \& Hall, 1st edition (1993).

[CZSS99] Y. Chiu, J. Zou, D. D. Stancil, and T. E. Schlesinger. Shape-optimized electrooptic beam scanners: analysis, design, and simulation. J. Lightwave Technol. 17(1), 108 - 114 (1999).

$\left[\mathrm{CKG}^{+} 13\right]$ A. Chmyrov, J. Keller, T. Grotjohann, M. Ratz, E. d'Este, S. Jakobs, C. Eggeling, and S. W. Hell. Nanoscopy with more than 100,000 'doughnuts'. Nat. Methods 10(8), $737-742$ (2013).

[CM96] J.-A. Conchello and J. G. McNally. Fast regularization technique for expectation maximization algorithm for optical sectioning microscopy. Proc. SPIE 2655, 199 - 208 (1996).

[Con18] Conoptics. Data Sheet Electro-Optic Beam Deflectors. https://www.conoptics. com/electro-optic-deflection-systems/ (2018). [Online; accessed 17December-2018].

$\left[\mathrm{DMB}^{+} 13\right]$ R. T. Darcy, D. McCloskey, K. E. Ballantine, B. D. Jennings, J. G. Lunney, P. R. Eastham, and J. F. Donegan. White light conical diffraction. Opt. Express 21(17), $20394-20403$ (2013).

[DH03] T. Dartigalongue and F. Hache. Precise alignment of a longitudinal Pockels cell for time-resolved circular dichroism experiments. J. Opt. Soc. Am. B 20(8), 1780 - 1787 (2003).

$\left[\mathrm{DKG}^{+} 15\right]$ E. D'Este, D. Kamin, F. Göttfert, A. El-Hady, and S. W. Hell. STED nanoscopy reveals the ubiquity of subcortical cytoskeleton periodicity in living neurons. Cell Rep. 10(8), 1246 - 1251 (2015).

[DCC $\left.{ }^{+} 19\right]$ J. Dreier, M. Castello, G. Coceano, R. Cáceres, J. Plastino, G. Vicidomini, and I. Testa. Smart scanning for low-illumination and fast RESOLFT nanoscopy in vivo. Nat. Commun. 10(1), 556 (2019).

[DKH05] M. Dyba, J. Keller, and S. W. Hell. Phase filter enhanced STED-4Pi fluorescence microscopy: theory and experiment. New J. Phys. 7, 134 (2005).

[EH05] A. Egner and S. W. Hell. Fluorescence microscopy with super-resolved optical sections. Trends Cell Biol. 15(4), 207 - 215 (2005).

[GAM] GAMDAN Optics. Potassium Dihydrogen Phosphate and Potassium Dideuterium Phosphate Datasheet. http://gamdan.com/catalog/kdp_ds.pdf. [Online; accessed 13-February-2019]. 
[Goo05] J. W. Goodman. Introduction to Fourier Optics. Roberts \& Company Publishers, 3rd edition (2005).

[Göt15] F. Göttfert. STED microscopy with scanning fields below the diffraction limit. Phd thesis, Max Planck Institute for Biophysical Chemistry, Göttingen, Germany (2015).

[GPH $\left.{ }^{+} 17\right]$ F. Göttfert, T. Pleiner, J. Heine, V. Westphal, D. Görlich, S. J. Sahl, and S. W. Hell. Strong signal increase in STED fluorescence microscopy by imaging regions of subdiffraction extent. Proc. Natl. Acad. Sci. U.S.A. 114(9), 2125 - 2130 (2017).

[Gus00] M. G. L. Gustafsson. Surpassing the lateral resolution limit by a factor of two using structured illumination microscopy. J. Microsc. 198(2), 82 - 87 (2000).

[GAS99] M. G. L. Gustafsson, D. A. Agard, and J. W. Sedat. $I^{5} m$ : $3 d$ widefield light microscopy with better than 100nm axial resolution. J. Microsc. 195(1), 10 - 16 (1999).

[Ham37] W. R. Hamilton. Third Supplement to an Essay on the Theory of Systems of Rays. Trans. R. Irish Acad. 17(1), 1 - 144 (1837).

[HKWL00] G. Hansson, H. Karlsson, S. Wang, and F. Laurell. Transmission measurements in KTP and isomorphic compounds. Appl. Opt. 39(27), 5058 - 5069 (2000).

[HKWL10] X. Hao, C. Kuang, T. Wang, and X. Liu. Effects of polarization on the de-excitation dark focal spot in STED microscopy. J. Opt. 12(11), 115707 (2010).

$\left[\mathrm{HKU}^{+} 08\right]$ B. Harke, J. Keller, C. K. Ullal, V. Westphal, A. Schönle, and S. W. Hell. Resolution scaling in STED microscopy. Opt. Express 16(6), 4154 - 4162 (2008).

[Här17] A. Härter. Ein Aufbau zur kohärenten Manipulation und zum zustandsabhängigen Transport einzelner Atome. Diploma thesis, Rheinische Friedrich-WilhelmsUniversität Bonn, Germany (2017).

[Hec05] E. Hecht. Optik. Oldenbourg Wissenschaftsverlag GmbH, 4th edition (2005).

$\left[\mathrm{HRH}^{+} 17\right]$ J. Heine, M. Reuss, B. Harke, E. D'Este, S. J. Sahl, and S. W. Hell. Adaptiveillumination STED nanoscopy. Proc. Natl. Acad. Sci. U.S.A. 114(37), 9797 - 9802 (2017).

[HJC02] R. Heintzmann, T. M. Jovin, and C. Cremer. Saturated patterned excitation microscopy - a concept for optical resolution improvement. J. Opt. Soc. Am. A 19(8), $1599-1609$ (2002). 
[HS92] S. Hell and E. H. K. Stelzer. Fundamental improvement of resolution with a 4Piconfocal fluorescence microscope using two-photon excitation. Opt. Commun. 93(56), $277-282$ (1992).

[Hel03] S. W. Hell. Toward fluorescence nanoscopy. Nat. Biotechnol. 21(11), $1347-1355$ (2003).

[Hel07] S. W. Hell. Far-Field Optical Nanoscopy. Science 316(5828), 1153 - 1158 (2007).

[Hel09] S. W. Hell. Microscopy and its focal switch. Nat. Methods 6(1), $24-32$ (2009).

[HK95] S. W. Hell and M. Kroug. Ground-state-depletion fluorescence microscopy: a concept for breaking the diffraction resolution limit. Appl. Phys. B 60(5), $495-497$ (1995).

[HSE09] S. W. Hell, R. Schmidt, and A. Egner. Diffraction-unlimited three-dimensional optical nanoscopy with opposing lenses. Nat. Photon. 3(7), 381 - 387 (2009).

[HW94] S. W. Hell and J. Wichmann. Breaking the diffraction resolution limit by stimulated emission: stimulated-emission-depletion fluorescence microscopy. Opt. Lett. 19(11), 780 - 782 (1994).

[HEJH05] M. Hofmann, C. Eggeling, S. Jakobs, and S. W. Hell. Breaking the diffraction barrier in fluorescence microscopy at low light intensities by using reversibly photoswitchable proteins. Proc. Natl. Acad. Sci. U.S.A. 102(49), 17565 - 17569 (2005).

[HBZ09] B. Huang, M. Bates, and X. Zhuang. Super-Resolution Fluorescence Microscopy. Annu. Rev. Biochem. 78, 993 - 1016 (2009).

[IYTS09] T. Imai, S. Yagi, S. Toyoda, and M. Sasaura. Fast Varifocal Lenses Based on $K T a_{1-x} \mathrm{Nb}_{x} \mathrm{O}_{3}$ (KTN) Single Crystals. NTT Technical Rev. 7(12), 1 - 5 (2009).

[JM76] D. P. Johnson and H. Matheson. An Analysis of Inertial Seisometer-Galvanometer Combinations. National Bureau of Standards, Washington D.C. (1976).

[KB08] T. K. Kalkandjiev and M. A. Bursukova. Conical refraction: an experimental introduction. Proc. SPIE 6994, 69940B (2008).

[KWR ${ }^{+}$06] R. J. Kittel, C. Wichmann, T. M. Rasse, W. Fouquet, M. Schmidt, A. Schmid, D. A. Wagh, C. Pawlu, R. R. Kellner, K. I. Willig, S. W. Hell, E. Buchner, M. Heckmann, and S. J. Sigrist. Bruchpilot Promotes Active Zone Assembly, $\mathrm{Ca}^{2+}$ Channel Clustering and Vesicle Release. Science 312(5776), 1051 - 1054 (2006).

[KEH01] T. Klar, E. Engel, and S. W. Hell. Breaking Abbe's diffraction resolution limit in fluorescence microscopy with stimulated emission depletion beams of various shapes. Phys. Rev. E 64(6 Pt. 2), 066613 (2001). 
[Krü17] J. Krüger. Tomographic STED microscopy. PhD thesis, University Göttingen, Germany (2017).

[LC05] J. W. Lichtman and J.-A. Conchello. Fluorescence microscopy. Nat. Methods 2(12), $910-919$ (2005).

[Lif15] Life Technologies. Fluospheres(B)carboxylate-modified microspheres. https://www. lifetechnologies.com/order/catalog/product/F8782 (2015). [Online; accessed 06-August-2015].

[LW53] E. H. Linfoot and E. Wolf. Diffraction Images in Systems with an Annular Aperture. Proc. Phys. Soc. B 66(2), 145 (1953).

[Llo33] H. Lloyd. On the phenomena presented by light in its passage along the axes of biaxial crystals. Phil. Mag. 1, 112 - 120, 207 - 210 (1833).

[Luc74] L. B. Lucy. An iterative technique for the rectification of observed distributions. Astron. J. 79(6), $745-754$ (1974).

$\left[\right.$ LRD $\left.^{+} 14\right]$ G. Lukinavičius, L. Reymond, E. D'Este, A. Masharina, F. Göttfert, H. Ta, A. Güther, M. Fournier, S. Rizzo, H. Waldmann, C. Blaukopf, C. Sommer, D. W. Gerlich, H.-D. Arndt, S. W. Hell, and K. Johnsson. Fluorogenic probes for live-cell imaging of the cytoskeleton. Nat. Methods 11(7), 731 - 733 (2014).

[MS04] G. F. Marshall and G. E. Stutz. Handbook of Optical and Laser Scanning. CRC Press, New York, 1st edition (2004).

[Min61] M. Minsky. Microscopy apparatus (1961). US Patent 3013467A.

[ME10] C. B. Müller and J. Enderlein. Image Scanning Microscopy. Phys. Rev. Lett. 104(19), 198101 (2010).

[NC09] U. Noomnarm and R. M. Clegg. Fluorescence lifetimes: fundamentals and interpretations. Photosynth. Res. 101(2-3), 181 - 194 (2009).

[NTT19] NTT Advanced Technology Corporation. KTN* Varifocal lens. http: //www.amstechnologies.com/fileadmin/amsmedia/downloads/5176_ varifocallensdatasheet.pdf (2019). [Online; accessed 25-November-2019].

[Nye04] J. F. Nye. Physical Properties of Crystals - Their Representation by Tensors and Matrices. Oxford University Press (2004).

[OP99] M. A. Omary and H. H. Patterson. Luminescence Theory. In Encyclopedia of Spectroscopy and Spectrometry, pages 1372 - 1391. Elsevier Ltd., 2nd edition (1999). 
[Per13] R. Persson. Breaking the diffraction limit using conical diffraction in super resolution fluorescence microscopy. Master's thesis, Royal Institute of Technology (KTH), Stockholm, Sweden (2013).

[POR $\left.{ }^{+} 09\right]$ C. F. Phelan, D. P. O'Dwyer, Y. P. Rakovich, J. F. Donegan, and J. G. Lunney. Conical diffraction and Bessel beam formation with a high optical quality biaxial crystal. Opt. Express 17(15), 12891 - 12899 (2009).

[PDL84] D. W. Pohl, W. Denk, and M. Lanz. Optical stethoscopy: Image recording with resolution $\lambda$ /20. Appl. Phys. Lett. 44(7), $651-653$ (1984).

[RW59] B. Richards and E. Wolf. Electromagnetic diffraction in optical systems II. Structure of the image field in an aplanatic system. Proc. R. Soc. A 253(1274), 358 379 (1959).

[Ric72] W. H. Richardson. Bayesian-Based Iterative Method of Image Restoration. J. Opt. Soc. Am. 62(1), 55 - 59 (1972).

[RB14] G. R. B. E. Römer and P. Bechtold. Electro-optic and acousto-optic laser beam scanners. Physics Procedia 56, 29 - 39 (2014).

[RBZ06] M. J. Rust, M. Bates, and X. Zhuang. Sub-diffraction-limit imaging by stochastic optical reconstruction microscopy (STORM). Nat. Methods 3(10), 793 - 795 (2006).

[SHJ17] S. J. Sahl, S. W. Hell, and S. Jakobs. Fluorescence nanoscopy in cell biology. Nat. Rev. Mol. Cell. Biol. 18(11), 685 - 701 (2017).

[ST07] B. E. A. Saleh and M. C. Teich. Fundamentals of Photonics. Wiley \& Sons, Inc., 2nd edition (2007).

[SIU $\left.{ }^{+} 08\right]$ L. Shao, B. Isaac, S. Uzawa, D. A. Agard, J. W. Sedat, and M. G. L. Gustafsson. $I^{5}$ s: Wide-Field Light Microscopy with 100-nm-Scale Resolution in Three Dimensions. Biophys. J. 94(12), $4971-4983$ (2008).

[SER $\left.{ }^{+} 11\right]$ T. Staudt, A. Engler, E. Rittweger, B. Harke, J. Engelhardt, and S. W. Hell. Farfield optical nanoscopy with reduced number of state transition cycles. Opt. Express 19(6), 5644 - 5657 (2011).

[Uni13] United Crystals. Properties of KTP single crystal. http://www . unitedcrystals. com/KTPProp.html (2013). [Online; accessed 26-January-2015].

[Val01] B. Valeur. Molecular Fluorescence: Principles and Applications. Wiley-VCH Verlag, Weinheim (2001).

[Vin15] B. Vinçon. Ultrafast switchable depletion patterns for STED microscopy generated by conical diffraction. Master's thesis, University Göttingen, Germany (2015). 
[VKS ${ }^{+}$08] J. Vogelsang, R. Kasper, C. Steinhauer, B. Person, M. Heilemann, M. Sauer, and P. Tinnefeld. A Reducing and Oxidizing System Minimizes Photobleaching and Blinking of Fluorescent Dyes. Angew. Chem. Int. Ed. Engl. 47(29), 5465 - 5469 (2008).

[WRO78] K. Weber, P. C. Rathke, and M. Osborn. Cytoplasmic microtubular images in glutaraldehyde-fixed tissue culture cells by electron microscopy and by immunofluorescence microscopy. Proc. Natl. Acad. Sci. USA 75(4), 1820 - 1824 (1978).

[WRL $\left.{ }^{+} 08\right]$ V. Westphal, S. O. Rizzoli, M. A. Lauterbach, D. Kamin, R. Jahn, and S. W. Hell. Video-Rate Far-Field Optical Nanoscopy Dissects Synaptic Vesicle Movement. Science 320(5873), 246 - 249 (2008).

[WS84] T. Wilson and C. J. R. Sheppard. Theory and Practice of Scanning Optical Microscopy. Academic Press, New York, 1st edition (1984).

[YF14] S. Yagi and K. Fujiura. Electro-optic KTN devices. Physics Procedia 56, 40 - 47 (2014).

[ZLLL09] S. Zeng, Q. Luo, D. Li, and X. Lü. Femtosecond pulse laser scanning using acoustooptic deflector. Sci. China Ser. G-Phys. Mech. Astron. 52(5), 685 - 692 (2009). 



\section{Nomenclature and Abbreviations}

\section{General nomenclature}

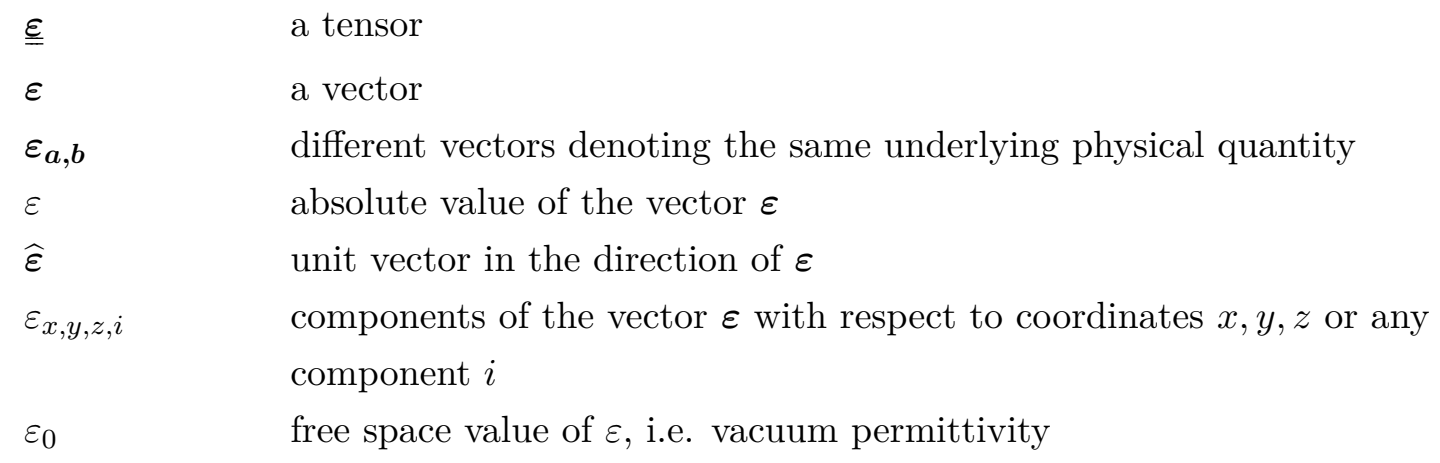

\section{Roman symbols and abbreviations}

$a$

$A$

$a_{1}, a_{2}$

$\mathrm{AD}^{*} \mathrm{P}$

$\mathrm{AOM}$

$\mathrm{APD}$

$B$

$B_{0}, B_{1}$

$\mathrm{BC}$

$c$

CCD

$d$

$d_{x}, d_{y}$

$\mathrm{DM}$

$d T$

$D$

$\mathcal{D}$

DyMIN

e

$\boldsymbol{E}$

EOD

Exc.

$f$

F crystal's aperture, also: pattern steepness

semiangle for the cone of conical diffraction, also: focal area

coefficients for the Taylor expansion of the refractive index

ammonium dideuterium phosphate

acousto-optic modulator

avalanche photo diode

magnetic induction

integral functions for the theory of conical diffraction

biaxial crystal

speed of light in vacuum

charge-coupled device

distance, displacement

components of the polarization vector in the Jones formalism

dichroic mirror

fraction of the pixel dwell time for RESCue imaging

electric displacement field

transverse profile of conically diffracted beam

Dynamic Intensity Minimum

Euler's number

electric field vector

electro-optic deflector

excitation

focal length, also: radial polar coordinate

Richardson-Lucy result 


\begin{tabular}{|c|c|}
\hline FIFO & First In First Out \\
\hline FPGA & Field-Programmable Gate Array \\
\hline FWHM & Full Width at Half Maximum \\
\hline$G$ & image \\
\hline GS & galvanometer scanner \\
\hline GT & Glan-Thompson prism \\
\hline$h_{\mathrm{STED}, \mathrm{conf}, \mathrm{eff}, j}$ & respective Point Spread Function \\
\hline$h_{P}$ & Planck constant \\
\hline $\boldsymbol{H}$ & magnetic field strength \\
\hline$H$ & matrix operator for the description of conical diffraction \\
\hline$i$ & imaginary unit \\
\hline$I$ & intensity \\
\hline$J$ & Jones vector \\
\hline$J_{0}, J_{1}$ & Bessel functions of zeroth and first order \\
\hline$J_{\mathrm{STED}, \mathrm{sat}}$ & pulse fluence \\
\hline$k$ & wave vector \\
\hline$k$ & absolute value of the wave vector, also: resolution enhancement, also: rate \\
\hline $\mathrm{KD}^{*} \mathrm{P}$ & potassium dideuterium phosphate \\
\hline KTP & potassium titanyl phosphate \\
\hline$l, L$ & length of: crystal, Pockels cell, medium \\
\hline$l T h$ & lower threshold for RESCue acquisition \\
\hline$m$ & slope of the scan system \\
\hline$M$ & magnification \\
\hline$\underline{\underline{M}}$ & matrix \\
\hline $\mathcal{M}$ & material constant for acousto-optic modulators \\
\hline$n$ & refractive index \\
\hline$N$ & $\begin{array}{l}\text { number of pixels, also: number of pattern orientations, } \\
\text { also: number of photons per pulse }\end{array}$ \\
\hline NA & numerical aperture \\
\hline$O$ & object function \\
\hline $\mathrm{OL}$ & objective lens \\
\hline OTF & Optical Transfer Function \\
\hline$P$ & transverse wave vector \\
\hline$P$ & $\begin{array}{l}\text { absolute value of the transverse wave vector, also: Pivot point, } \\
\text { also: laser power }\end{array}$ \\
\hline $\mathrm{PC}$ & Pockels cell \\
\hline $\mathrm{PH}$ & pinhole \\
\hline PSA & Polarization State Analyzer \\
\hline PSF & Point Spread Function \\
\hline
\end{tabular}




\begin{tabular}{|c|c|}
\hline PSG & Polarization State Generator \\
\hline$p T$ & pixel dwell time \\
\hline$p x$ & pixel size \\
\hline$Q$ & integration variable \\
\hline$R$ & radius, radial coordinate \\
\hline$R_{0}$ & radius of the emergent cone of conical diffraction \\
\hline $\mathfrak{r}$ & linear electro-optic coefficient \\
\hline $\mathcal{R}$ & intensity ratio for acousto-optic modulators \\
\hline$\Re$ & real part of a complex number \\
\hline RESCue & REduction of State transition Cycles \\
\hline RESOLFT & REversible Saturable OpticaL Fluorescence Transitions \\
\hline$s$ & direction of wave normal, $\boldsymbol{k}=k \boldsymbol{s}$ \\
\hline$S$ & Poynting vector \\
\hline $\mathfrak{s}$ & quadratic electro-optic coefficient \\
\hline S & sample \\
\hline$S_{0}$ & ground state \\
\hline$S_{1}$ & first excited state \\
\hline SNR & signal-to-noise ratio \\
\hline STED & STimulated Emission Depletion \\
\hline$t$ & time \\
\hline$T$ & delay in the scan system \\
\hline$T$ & Jones matrix \\
\hline$T_{1}$ & triplet state \\
\hline $\mathrm{TC}$ & telescope \\
\hline TTL & transistor-transistor logic \\
\hline$U$ & voltage \\
\hline$u T h$ & upper threshold for RESCue acquisition \\
\hline$u, v$ & coordinates in Fourier space \\
\hline$v_{p}$ & phase velocity \\
\hline$w$ & beam waist \\
\hline$W$ & object's function \\
\hline$x, X$ & spatial coordinates \\
\hline$y, Y$ & spatial coordinates \\
\hline$z, Z$ & spatial coordinates \\
\hline
\end{tabular}




\section{Greek symbols}

$\alpha$

$\alpha_{R c h}$

$\beta$

$\gamma$

$\Gamma$

$\delta$

$\Delta$

$\Delta x, \Delta t$

$\underline{\underline{\varepsilon}}$

$\zeta$

$\eta$

$\theta$

$\Theta$

$\kappa$

$\lambda$

$\Lambda$

$\underline{\underline{\mu}}$

$\pi$

$\rho$

$\rho_{0}$

$\sigma$

$\tau$

$\varphi$

$\phi$

$\chi$

$\Psi$

$\omega$

angle

regularization parameter for Richardson-Lucy deconvolution

ratio between $k_{1 \mathrm{D}}$ and $k_{2 \mathrm{D}}$

calibration parameter for the scanning device, also: optical cross section

phase shift

angle of the polarizer for conical diffraction

difference of two values, also: Full Width at Half Maximum

pixel size and dwell time

electric permittivity tensor

scaled axial coordinate for conical diffraction, also: saturation factor

parameter for description of electro-optic deflectors, also: suppression factor

angle, angle of incidence

Heaviside function

material constant of the electro-optic deflectors

wavelength of an optical wave

wavelength of an acoustic wave

permeability tensor

Ludolph's number

scaled radial coordinate for conical diffraction

parameter for conical diffraction

standard deviation

lifetime

phase angle

angle polar coordinates

ellipticity

polarization angle

angular frequency of an optical wave 


\section{A. Appendix}

\section{Mathematical description of a galvanometer}

A galvanometer is an instrument typically employed for detecting small electric currents. Its central part is a coil in a constant magnetic field with the current flowing through this coil [MS04. By the induced magnetic field, the coil twists, resulting in a change of the pointer's angular position. Typically, the movement is adequately damped, yielding a fast settling at the new position, and an appropriate design of the system yields a linear dependence of the caused rotation angle on the applied electric current [MS04].

Galvanometers are not only employed for detecting electric current, but also as galvo-motors to drive rotational movements precisely by applying an electric voltage. The equations for describing the movement of such a device in dependence on the applied voltage $U(t)$ are given by [JM76]

$$
\begin{aligned}
0 & =J \frac{\mathrm{d}^{2} \theta}{\mathrm{d} t^{2}}+r_{\theta} \frac{\mathrm{d} \theta}{\mathrm{d} t}+K \theta-G \cdot I(t), \\
U(t) & =G \frac{\mathrm{d} \theta}{\mathrm{d} t}+L \frac{\mathrm{d} I}{\mathrm{~d} t}+R \cdot I(t) .
\end{aligned}
$$

Here, no mechanical torque is assumed to be applied to the galvanometer coil, and the galvanometer is supposed to be fixed in space. $J$ denotes the moment of inertia of the coil, $r_{\theta}$ is the mechanical damping constant, which is assumed to be constant in the following, $K$ is the stiffness of the coil suspension and $G$ is the galvanometer constant. Furthermore, $I(t)$ is the resulting current in the coil, including applied and induced current, $L$ denotes the electrical inductance of the coil and $R$ is the electrical resistance of the coil. Analogously to the analysis in JM76, $L$ is presumed to be negligible, allowing the elimination of $I(t)$ from the above equations. This yields

$$
J \frac{\mathrm{d}^{2} \theta}{\mathrm{d} t^{2}}+\left(r_{\theta}+\frac{G^{2}}{R}\right) \frac{\mathrm{d} \theta}{\mathrm{d} t}+K \theta=\frac{G}{R} U(t)
$$

For a standard image scan, as presented in subsection 3.5. the applied voltage is varied linearly with time, i.e. $U(t)=m \cdot t$. Hence, equation A.1 rewrites as

$$
\frac{\mathrm{d}^{2} \theta}{\mathrm{d} t^{2}}+K_{1} \frac{\mathrm{d} \theta}{\mathrm{d} t}+K_{2} \theta=K_{3} \cdot m \cdot t
$$

with $K_{1}:=\left(r_{\theta}+\frac{G^{2}}{R}\right) / J, K_{2}:=K / J$ and $K_{3}:=G /(R J)$. A general solution to this differential equation is given by the sum of the general solution of the homogeneous differential equation and a particular solution of the nonhomogeneous equation. The corresponding homogeneous 
equation

$$
\frac{\mathrm{d}^{2} \theta}{\mathrm{d} t^{2}}+K_{1} \frac{\mathrm{d} \theta}{\mathrm{d} t}+K_{2} \theta=0
$$

is the differential equation of a damped harmonic oscillator. A galvanometer, as described by this equation, should quickly reach its angular position without oscillating or drastically overshooting. Hence, the system can be assumed to be critically damped, i.e.

$$
K_{1}=2 \sqrt{K_{2}}
$$

In this case, the general solution to equation A.3 is

$$
\theta(t)=A_{1} e^{-\sqrt{K_{2}} t}+A_{2} \cdot t \cdot e^{-\sqrt{K_{2}} t}
$$

with $A_{1}, A_{2}$ being constants. For solving the nonhomogeneous equation (A.2), the ansatz $\theta(t)=\alpha_{0}+\alpha_{1} t$ is employed. A comparison of coefficients yields

$$
\alpha_{1}=\frac{K_{3} m}{K_{2}}, \quad \alpha_{0}=-\frac{K_{1} K_{3} m}{K_{2}^{2}}=-\frac{2 K_{3} m}{K_{2}^{3 / 2}} .
$$

This yields the general solution to equation A.2

$$
\theta(t)=A_{1} e^{-\sqrt{K_{2}} t}+A_{2} \cdot t \cdot e^{-\sqrt{K_{2}} t}-\frac{2 K_{3} m}{K_{2}^{3 / 2}}+\frac{K_{3} m}{K_{2}} t
$$

Since the galvanometer is at rest for $t=0, \theta(0)=0$ and $\frac{\mathrm{d} \theta}{\mathrm{d} t}(0)=0$ need to be fulfilled. Consequently, $A_{1}=\frac{2 K_{3} m}{K_{2}^{3 / 2}}$ and $A_{2}=\frac{K_{3} m}{K_{2}}$ hold and thus

$$
\theta(t)=\frac{2 K_{3} m}{K_{2}^{3 / 2}} e^{-\sqrt{K_{2}} t}+\frac{K_{3} m}{K_{2}} \cdot t \cdot e^{-\sqrt{K_{2}} t}-\frac{2 K_{3} m}{K_{2}^{3 / 2}}+\frac{K_{3} m}{K_{2}} t \underset{\text { large } t}{\longrightarrow} \frac{K_{3} m}{K_{2}}\left(t-\frac{2}{\sqrt{K_{2}}}\right) .
$$

For an ideal, instantaneously reacting galvanometer, the angular position can be derived from considering equation A.1 for the stationary case, i.e.

$$
\theta_{\text {ideal }}=\frac{K_{3}}{K_{2}} U
$$

A comparison with $\theta(t)$ for $U(t)=m \cdot t$ yields

$$
\theta\left(t+\frac{2}{\sqrt{K_{2}}}\right) \underset{\text { large }}{\rightarrow} \theta_{\text {ideal }}(t)
$$

corresponding to a constant offset in time between ideal and actual angular position, which depends on the physical characteristics of the employed galvanometer coil. 


\section{B. Appendix}

\section{Sample preparation}

Samples of fluorescent microspheres (FluoSpheres carboxylate-modified microspheres, $48 \mathrm{~nm}$ actual diameter, crimson fluorescent (625/645); Life Technologies, USA) are typically employed as a first control for the setup's performance. A solution of microspheres is diluted $1: 5,000$ with purified water, incubated on a coverslip coated with Poly-L-lysine $\left(0.1 \%(\mathrm{w} / \mathrm{v})\right.$ in $\mathrm{H}_{2} \mathrm{O}$; Sigma-Aldrich, USA) for adhesion and subsequently embedded in self-prepared Mowiol. For visualizing the Point Spread Functions (PSFs), $150 \mathrm{~nm}$ sized reflective gold beads (BBI Solutions, United Kingdom) are used, following a similar incubation and embedding procedure as for the fluorescent microspheres.

Cell measurements are conducted on cells fixed in $-20^{\circ} \mathrm{C}$ cold methanol or in $8 \%$ PFA (paraformaldehyde, Science Services GmbH, Germany) in PBS (phosphate buffered saline, SigmaAldrich, USA). Immunostaining of the structures of interest is performed with Abberior STAR 635P (Abberior GmbH, Germany) or Abberior STAR RED (Abberior GmbH, Germany) as fluorescent dye coupled to a suitable secondary antibody. Depending on the application, anti$\alpha$-tubulin from mouse (T6074, Sigma-Aldrich, USA), anti- $\alpha$-tubulin from rabbit (ab18251, Abcam, UK), anti-vimentin from mouse (V6389, Sigma-Aldrich, USA) or anti-PMP70 from mouse (SAB4200181, Sigma-Aldrich, USA) is chosen as primary antibody. 2\% BSA (bovine serum albumin fraction V, Carl Roth GmbH, Germany) in PBS is used as blocking agent between incubation steps. The sample is subsequently embedded in self-prepared Mowiol.

Actin filaments in vitro are prepared by diluting monomeric G-actin (Hypermol, Germany) to a concentration of $1 \mu \mathrm{g} / \mathrm{ml}$ in PolyMix (Hypermol, Germany), which initiates the polymerization of the G-actin into the filamentous F-actin. The addition of Silicon Rhodamine (SiR, Spirochrome, Switzerland) as fluorescent dye yields a staining of the polymerized F-actin filaments. The solution is incubated on a coverslip for $1 \mathrm{~min}$ with a subsequent washing step performed with MonoMix (Hypermol, Germany). Finally, the sample is embedded in selfprepared Mowiol.

For imaging of living cells, the cells are seeded on cover glasses until the desired confluency is reached. The cell culture medium is replaced by the staining solution of $500 \mathrm{nmol} \mathrm{SiR-tubulin}$ (Spirochrome, Switzerland) in cell culture medium and the sample is incubated for three hours at $37^{\circ} \mathrm{C}$ and $5 \% \mathrm{CO}_{2}$. Subsequently, the staining medium is removed and the sample is embedded on a glass slide with a well in $\approx 50 \mu$ l of DMEM ${ }^{\text {ffp }}-2$ (Evrogen, Russia). 



\section{Acknowledgments}

After years of work and effort it is time to acknowledge all the help, assistance and encouragement I received from numerous sides and persons, whom I owe my deep gratitude. Without the scientific, non-scientific and moral support of colleagues, family and friends my PhD time would have been much less enjoyable and successful.

I'm grateful to my supervisor and primary advisor apl. Prof. Dr. Alexander Egner for giving me the opportunity to work on my $\mathrm{PhD}$ thesis in a very well-equipped, international laboratory group. Never tired to think about possible pathways and applications, you set the basis for many fruitful discussions leading to plenty of new approaches and ideas.

I'd like to thank Prof. Dr. Sarah Köster as my secondary advisor. Thank you for your encouragement, for your interest in the project and for providing advice, suggestions and help whenever I asked for.

I moreover thank Prof. Dr. Jörg Enderlein, Prof. Dr. Wolfram Kollatschny, Prof. Dr. Silvio O. Rizzoli and apl. Prof. Dr. Michael Seibt for spending the time and effort to complete my thesis committee.

My sincere gratitude is owed to Dr. Claudia Geisler for supervision as well as proof-reading of the thesis. Thank you for myriads of suggestions and ideas both in the experimental work as well as in programming or analyzing data. No matter when I asked for, you always found the time to listen to any stupid problem, even if it was a mouse to be let into freedom. Thank you for your enthusiasm about little steps towards success and for making lunch breaks more enjoyable by bringing up other interesting topics than scientific ones.

Furthermore, I'd like to thank Jaydev Jethwa, who - as he already did during my master's thesis - helped me whenever high voltages came into play. Thanks to you I'm still healthy and alive and haven't burnt down the institute neither.

High-resolution microscopy would not make sense without adequate samples. My gratitude goes to Blanka Hampe as well as Jasmin Rehman for providing fixed cells and giving suggestions and ideas on how to improve my antibody-stainings. Thank you for the very uncomplicated communication and coordination. Moreover, I owe my deep gratitude to Dr.

Kareem Soliman for keeping the cell culture running only for me and for providing fixed and living cells whenever I asked for.

A special thanks goes to Maik Lübbecke for his help with and realization of the printed circuit board of the scan system's control. Thank you for your time and effort in discussing possible designs, changing them following new experimental insights and finally giving a useful hand in debugging the system. Thank you for always being positive and for being interested in the outcome of my experiments.

Furthermore, I thank Dr. Andreas Schönle and Dr. Jan Keller-Findeisen for providing 
the MATLAB code for the PSF calculation.

I'd like to express my gratitude to all colleagues at the LLG, especially the administration for a professional handling of all bureaucratic issues as well as the mechanical workshop for realizing all adapters, mounting constructions and shieldings not available on the market. I'm grateful to all former and present members of the Optical Nanoscopy group for providing a productive working environment.

Parul and Debadrita, thank you for giving a lot of insights into Indian culture and food and for always being ready to discuss scientific problems as well as the next birthday present.

Kareem, apart from keeping the cells alive, thank you for your suggestions and discussions on possible structures of interest for the different applications.

Special thanks go to René for introducing me to lab work in the beginning and for being a constant source of jokes and good humor. Thank you for always being ready to discuss any problems and for never getting tired of searching lost components in your laboratory. Thank you for many game evenings, escape and hiking adventures, which successfully distracted me from thinking about work-related problems.

I'm particularly thankful to Julia: Thank you for sharing the office and lunch breaks with me and for tolerating me in any mood. Thank you for listening and discussing problems, scientific and non-scientific ones, and for cheering me up whenever things were not going ahead like I'd wished for. You became a close friend of mine, sharing a lot of theater and concert evenings as well as trying to understand the theory and practice of folding (non-perfect, but nice-looking) origami art-work.

Not only scientific support was crucial to make this thesis become real. Without close friends, who knew to distract me whenever necessary, I wouldn't be where I am today. I'd like to thank Hanna, Claudia and Bettina for the study time together, including a lot of card game evenings as well as outdoor adventures, and for keeping in touch also afterwards. A special thanks goes to Damaris for introducing me to math in the very beginning, encouraging me to pursue this path and moreover for being a very close and constant friend for more than a decade now - regardless of the often huge spatial distance.

I'd moreover like to thank everyone whom I had the honor to play music with, particularly Nora. Thank you for your enthusiasm, for being positive and full of energy and for updating me and inviting me to all your concerts. Special thanks also go to Jasmin, Hanna and Cornelius, with whom I've had the chance to rehearse for more than five years now. Thank you for never starting a rehearsal without asking how it's going and for distracting me with Beethoven, Brahms or Schubert from any possible kind of non-music problem.

Jenny and Carsten, thank you for being such close, encouraging and supporting friends. Thank you for taking care of me, for distracting me from work, but for also listening to me whenever problems were too big to be simply pushed aside. Jenny, thank you for all your sup- 
port, smile and happiness, and thank you for proof-reading parts of this thesis in your parental leave. Thank you both for keeping in touch and for sharing unforgettable memories.

My deep gratitude is owed to those persons knowing me the longest - my parents and siblings. Thank you for making me the person I am, for believing in me and for trusting me to take the correct decisions even though you were not always familiar with the details of what I was doing. Thank you for always having an open ear for any not too subject-specific problem and for listening to all my complaints about things you had no chance to change. Thank you for your constant support and encouragement.

Last, but definitely not least, thank you, Francesco, first of all for your tenacity and for buying a panettone with me. Thank you for listening to and discussing scientific problems at literally any time of the day (and night) and for always being there with me and encouraging me regardless of the decisions I took and I was about to take. Thank you for believing in me especially when I didn't and for introducing me to the Thai Chi mood, just to mention one out of an uncountable amount of things. Thank you for your unconditional support and for being the person you are. 



\section{Eidesstattliche Erklärung}

Hiermit erkläre ich, dass ich die vorliegende Arbeit selbstständig angefertigt, nicht anderweitig zu Prüfungszwecken vorgelegt und keine anderen als die angegebenen Hilfsmittel verwendet habe. Sämtliche wissentlich verwendeten Textausschnitte, Zitate oder Inhalte anderer Verfasser wurden ausdrücklich als solche gekennzeichnet. 



\section{List of publications related to doctoral dissertation}

Parts of this thesis have been published as follows

1. B. Vinçon, C. Geisler, and A. Egner. Pixel hopping enables fast STED nanoscopy at low light dose. Opt. Express 28(4), 4516-4528 (2020). 